Sandro Luis Vatanabe

Projeto de Materiais Piezocompósitos Baseados no Conceito de Gradação Funcional Utilizando o Método de Otimização Topológica 
Sandro Luis Vatanabe

\section{Projeto de Materiais Piezocompósitos Baseados no Conceito de Gradação Funcional Utilizando o Método de Otimização Topológica}

Tese apresentada à Escola Politécnica da Universidade de São Paulo para obtenção do Título de Doutor em Engenharia Mecânica. 


\section{Projeto de Materiais Piezocompósitos Baseados no Conceito de Gradação Funcional Utilizando o Método de Otimização Topológica}

Tese apresentada à Escola Politécnica da Universidade de São Paulo para obtenção do Título de Doutor em Engenharia Mecânica.

Área de concentração:

Engenharia de Controle e Automação Mecânica

Orientador:

Prof. Dr. Emílio Carlos Nelli Silva 
Este exemplar foi revisado e alterado em relação à versão original, sob
responsabilidade única do autor e com a anuência de seu orientador

São Paulo, 12 de dezembro de 2012.

Assinatura do autor brob tuis lotanele

Assinatura do orientador

Vatanabe, Sandro Luis

Projeto de materiais piezocompósitos baseados no conceito de gradação funcional utilizando o método de otimização topológica / S.L. Vatanabe. -- ed.rev. -- São Paulo, 2012.

$168 \mathrm{p}$.

Tese (Doutorado) - Escola Politécnica da Universidade de São Paulo. Departamento de Engenharia Mecatrônica e de Sistemas Mecânicos.

1. Método dos elementos finitos 2. Materiais compósitos 3. Cerâmica piezelétrica 4. Métodos topológicos (Otimização) I. Universidade de São Paulo. Escola Politécnica. Departamento de Engenharia Mecatrônica e de Sistemas Mecânicos II. t. 
A meus pais. 


\section{Agradecimentos}

Ao meu orientador, Prof. Dr. Emílio C. N. Silva, agradeço pela atenção dispensada, discussões estimuladas e sugestões dadas durante todo o período de elaboração deste trabalho. Os anos de convívio me proporcionaram enorme amadurecimento acadêmico, profissional e pessoal.

Ao Prof. Dr. Glaucio H. Paulino, agradeço pela colaboração nas publicações dos artigos e principalmente pelo incentivo e motivação para o desenvolvimento desta pesquisa.

À EPUSP (Escola Politécnica da Universidade de São Paulo) por disponibilizar toda a infraestrutura necessária para o desenvolvimento desta pesquisa.

À FAPESP (Fundação de Amparo à Pesquisa do Estado de São Paulo) pela concessão da bolsa de doutorado, viabilizando a realização deste trabalho.

Ao CNPq (Conselho Nacional de Desenvolvimento Científico e Tecnológico) pelo apoio financeiro neste trabalho.

Minha sincera gratidão à minha mãe Fujika, ao meu pai Luiz Carlos e à minha irmã Francina, pela educação que vocês me deram, pela formação que vocês me proporcionaram e pelo apoio incondicional em todos os meus sonhos.

À Valência, agradeço pelo amor e apoio incondicional, e à sua mãe Elisa, pelo apoio ao longo deste trabalho.

Aos amigos da pós-graduação Ronny, Marcão, Cesar, Guga, Wilfredo, Fausto, Polastro, Douglas, Amigo, Wagner, Teves, Ruben, Flávio e Langellotti, agradeço pelas discussões acadêmicas, amizade e companheirismo. 


\section{Resumo}

Um material piezocompósito é resultante da combinação de um material piezelétrico com outros materiais não-piezelétricos, oferecendo vantagens substanciais em relação aos materiais piezelétricos convencionais. Diferentes propriedades efetivas podem ser obtidas alterando-se a fração de volume dos constituintes ou a própria topologia da célula unitária do piezocompósito. Materiais com Gradação Funcional (MGF) são materiais compósitos avançados, projetados de tal forma que sua composição varie gradualmente numa direção espacial. A vantagem do conceito MGF é não apresentar interface convencional entre os materiais da inclusão e da matriz, reduzindo assim um problema comum em materiais compósitos laminados, como por exemplo, o surgimento de concentração de tensões mecânicas. O Método de Otimização Topológica (MOT) é uma técnica computacional utilizada para se determinar a distribuição de materiais em uma estrutura ou material de forma sistemática, a fim de se extremizar uma determinada função objetivo. Assim, esse trabalho propõe uma metodologia sistemática e genérica para o projeto de materiais piezocompósitos com gradação funcional (MPGF) utilizando o MOT, tanto para aplicações quasi-estáticas, quanto para aplicações dinâmicas. Dessa forma, divide-se o projeto de materiais piezocompósitos em três grupos. O primeiro grupo consiste em um método de projeto de materiais baseado na combinação do método de homogeneização com o MOT para o projeto de MPGF para aplicações quasi-estáticas, onde o objetivo é projetar materiais piezocompósitos que, de modo geral, maximizem a conversão de energia mecânica em elétrica. A aplicação utilizada como exemplo neste trabalho são materiais empregados em dispositivos de coleta de energia. O segundo grupo visa aplicações dinâmicas de materiais piezocompósitos fonônicos, onde a propriedade de interesse é a possibilidade de se ter faixas de frequência, mais conhecidas por band gaps, nas quais ondas elásticas não se propagam. Assim, neste estudo visa-se o projeto de MPGF fonônicos com largura e posição de band gaps prescritos, empregando estruturas unidimensionais, e a maximização de diversos band gaps, empregando estruturas bidimensionais. O terceiro grupo explora o conceito de gradação geométrica, baseado em repetições de padrão ao longo do domínio de projeto, porém cada repetição tem um ou mais comprimentos modificados, de forma gradual. Dessa forma, suas propriedades alteram-se progressivamente ao longo da estrutura, embora a distribuição de materiais seja discreta, contornando assim possíveis dificuldades de manufatura. Esta abordagem é empregada visando à aplicação na coleta de energia, onde se procura maximizar a potência elétrica gerada em um resistor acoplado aos eletrodos, através da obtenção da topologia otimizada de estruturas piezocompósitas. Exemplos numéricos são apresentados de forma a ilustrar as metodologias de projeto propostas, bem como, analisar a influência dos parâmetros de otimização nos resultados. 


\section{Abstract}

Piezocomposite materials result from the combination of a piezoelectric material with other non-piezoelectric materials, offering advantages over conventional piezoelectric materials. Different effective properties can be obtained by changing the volume fraction of constituent materials, the shape of inclusions, or even the topology of the unit cell. Functionally Graded Materials (FGM) are composite materials, which are designed so that its composition varies gradually in space. One of the advantages of FGMs is that there is no conventional interface between the constituent materials, which reduces, for instance, microscopic stress concentration problems in composite materials. Topology Optimization Method (TOM) is a computational technique used to determine the material distribution of a structure or material in a systematic way, in order to maximize a determined objective function. Thus, this study proposes a generic and systematic methodology to design Functionally Graded Piezocomposites Materials (FGPM) using TOM, for quasi-static and dynamic applications. The study is divided into three groups. The first group combines the homogenization method with TOM in order to design FGPM for quasi-static applications, where the goal is to maximize the conversion of mechanical energy into electrical energy. The application used as an example in this study focuses materials used in energy harvesting devices. The second group focuses on dynamic applications of phononic piezocomposite materials, where the property of interest is the possibility of having frequency band gaps, in which elastic waves do not propagate. This study aims to design phononic FGPM with prescribed band gap width using one-dimensional model, and to design phononic FGPM with maximized band gaps using two-dimensional model. The third group investigates the pattern gradation concept, based on pattern repetitions over the design domain, but each pattern has one or more dimensions gradually modified. Thus, properties change gradually along the structure, although the material distribution keeps in the discrete form, thereby circumventing potential manufacturing difficulties. The objective function consists of maximizing the electric power generated in a load resistor. A projection scheme is employed to compute the element densities from design variables and control the length scale of the material density. Numerical examples are presented and discussed using the proposed methods. 


\section{Conteúdo}

\section{Lista de Figuras}

\section{Lista de Tabelas}

Lista de Abreviaturas e Siglas

1 Introdução 1

1.1 Materiais com Gradação Funcional (MGF) . . . . . . . . . . . . . . . 3

1.2 Método de Otimização Topológica (MOT) . . . . . . . . . . . . . . . 4

1.3 Projeto de materiais piezocompósitos com gradação funcional para aplicações quasi-estáticas . . . . . . . . . . . . . . . . . . . 8

1.4 Projeto de materiais piezocompósitos fonônicos . . . . . . . . . . . . . 9

1.5 Projeto de estruturas piezocompósitas com gradação geométrica . . . . . . 10

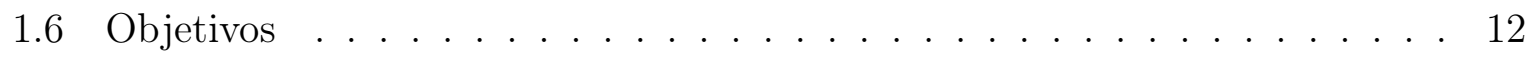

1.7 Justificativa . . . . . . . . . . . . . . . . . . . . 13

1.8 Contribuições Científicas . . . . . . . . . . . . . . . . . 14

1.9 Organização do Documento . . . . . . . . . . . . . . 15

2 Projeto de Materiais Piezocompósitos com Gradação Funcional para $\begin{array}{ll}\text { Aplicações Quasi-Estáticas } & 16\end{array}$

2.1 Fundamentação Teórica . . . . . . . . . . . . . . . . . 20

2.1.1 Método de Homogeneização . . . . . . . . . . . . . . . . . . . . 20

2.1.2 Cálculo das tensões mecânicas na microestrutura . . . . . . . . . . 24

2.2 Formulação do Problema de Otimização Topológica . . . . . . . . . . . . . 25

2.2 .1 Modelo de Material . . . . . . . . . . . . . . . . . . 27 
2.3 Implementação Numérica . . . . . . . . . . . . . . . . . . . . . . . . . . . . 29

2.3.1 Elementos Finitos Gradados . . . . . . . . . . . . . . . . . . . 29

2.3.2 Elementos finitos poligonais . . . . . . . . . . . . . . . 31

2.3.3 Método da homogeneização . . . . . . . . . . . . . . . . . 35

2.3.4 Otimização de Material Discreto (OMD) . . . . . . . . . . . . 40

2.3.5 Controle de Gradação de Material . . . . . . . . . . . . . . . . . . . 43

2.3.6 Análise de Sensibilidade . . . . . . . . . . . . . . . . . . . 44

2.3.7 Formulação do Problema na Forma Discreta . . . . . . . . . . . . . 50

2.4 Resultados . . . . . . . . . . . . . . . . . . . . 51

2.4.1 Projeto de materiais sem gradação de material . . . . . . . . . . . . 54

2.4.2 Projeto de materiais com gradação funcional . . . . . . . . . . . . . 57

2.5 Considerações Finais . . . . . . . . . . . . . . . . . . . . . . . 60

3 Projeto de Materiais Piezocompósitos com Gradação Funcional Visando Aplicações Fonônicas

3.1 Fundamentação Teórica . . . . . . . . . . . . . . . . . . . . 67

3.1.1 Modelo Unidimensional . . . . . . . . . . . . . . . . . . . 67

3.1.2 Modelo Bidimensional . . . . . . . . . . . . . . . . . . . 70

3.2 Formulação do Problema de Otimização Topológica . . . . . . . . . . . . . 73

3.2.1 Modelo Unidimensional . . . . . . . . . . . . . . . . . . . . 73

3.2 .2 Modelo Bidimensional . . . . . . . . . . . . . . . . . . . . 74

3.3 Implementação Numérica . . . . . . . . . . . . . . . . . . . . . . . . . . . . 75

3.4 Resultados . . . . . . . . . . . . . . . . . . . . 77

3.4 .1 Modelo Unidimensional . . . . . . . . . . . . . . . . . . . 77

3.4 .2 Modelo Bidimensional . . . . . . . . . . . . . . . . 83

3.5 Considerações Finais . . . . . . . . . . . . . . . . . . . . . . . 97

4 Projeto de Transdutores Piezocompósitos com Gradação Geométrica 99

4.1 Método de Elementos Finitos Dinâmico para Materiais Piezelétricos . . . . 100 
4.2 Formulação do Problema de Otimização Topológica . . . . . . . . . . . . . . 102

4.2 .1 Modelo de Material . . . . . . . . . . . . . . . . . . . . . 104

4.3 Implementação Numérica . . . . . . . . . . . . . . . . . . . . . . . . . . 104

4.3 .1 Cálculo das Sensibilidades . . . . . . . . . . . . . . . 107

4.4 Exemplo Numérico . . . . . . . . . . . . . . . . . . . . . . 108

4.4.1 Caso 1: Sem repetição de padrões . . . . . . . . . . . . . . . . 110

4.4 .2 Caso 2: Com repetição de padrão . . . . . . . . . . . . . . . . . . 110

4.4.3 Caso 3: Com gradação geométrica . . . . . . . . . . . . . . . . 111

4.4 .4 Resultados e Discussões . . . . . . . . . . . . . . . 111

4.5 Considerações Finais . . . . . . . . . . . . . . . . . . 115

5 Conclusões Finais $\quad 117$

5.1 Sugestões de Trabalhos Futuros . . . . . . . . . . . . . . . . . 119

$\begin{array}{ll}\text { Referências } & 122\end{array}$

Apêndice A - Materiais Piezelétricos $\quad 138$

Apêndice B - Método de Elementos Finitos (MEF) para Materiais

$\begin{array}{ll}\text { Piezelétricos } & 143\end{array}$

B.1 Formulação Variacional para Problemas Piezelétricos . . . . . . . . . . . . 143

B.2 Formulação de Elementos Finitos para Problemas Piezelétricos . . . . . . . 144

Apêndice C - Método de Homogeneização 148

C.1 Formulação Teórica . . . . . . . . . . . . . . . . . . . . . . . 148

C.2 Condições de simetria . . . . . . . . . . . . . . . . 157

Apêndice D - Verificação da Implementação do Método de $\begin{array}{lr}\text { Homogeneização } & 159\end{array}$

Apêndice E - Método de Otimização Topológica (MOT) 162

E.1 Domínio Estendido Fixo de Projeto . . . . . . . . . . . . . . . . . . 162 
E.2 Modelo de Material . . . . . . . . . . . . . . . . . . . 163

E.3 Aspectos Numéricos do MOT . . . . . . . . . . . . . . . . . . 165

E.3.1 Dependência de malha . . . . . . . . . . . . . . . . . . 165

E.3.2 Instabilidade de tabuleiro de xadrez . . . . . . . . . . . . . . 167 


\section{Lista de Figuras}

1.1 Exemplos de conectividade em materiais piezocompósitos: (a) piezocompósito 1-3 e (b) piezocompósito 2-2 . . . . . . . . . . . 2

1.2 Microfotografia de um Material com Gradação Funcional (MGF) (KIEBACK; NEUBRAND; RIEDEL, 2003). . . . . . . . . . . . . . . . . . 4

1.3 Exemplo de Material Piezocompósito com Gradação Funcional (MPGF). 4

1.4 Etapas do método de otimização topológica (MOT) no projeto da célula unitária de materiais compostos. . . . . . . . . . . . . . . . . 7

1.5 Exemplo de gradação geométrica. . . . . . . . . . . . . . . . . . . 11

1.6 Substituição da gradação de material pela gradação geométrica, no interior da célula unitária. . . . . . . . . . . . . . . . . . . . . . . . . 11

1.7 Exemplos de gradação geométrica. . . . . . . . . . . . . . . . . . . . . 12

2.1 Exemplo de material com (a) coeficiente de Poisson negativo; (b) coeficiente térmico de expansão nulo (SIGMUND, 2001b, 2001a; SIGMUND; TORQUATO,

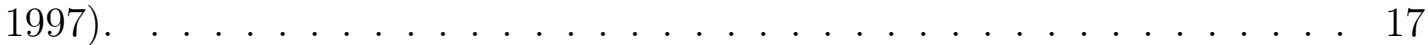

2.2 Imagem da matriz de células unitárias (WING; HALLORAN, 2006). . . . . . 19

2.3 Conceito de Multi-escala ilustrando as dimensões macro (x) e micro (y). . 22

2.4 Exemplo de aplicação de coleta de energia (KAWAI, 2006). . . . . . . . . . 26

2.5 Modelo utilizado para definir os termos de $k \ldots \ldots \ldots \ldots$

2.6 Esquemático do conceito clássico de otimização de orientação de polarização numa malha de elementos finitos. As setas indicam as direções de polarização em cada elemento, definidas pelas variáveis de projeto $\left(\theta_{i}\right) .28$

2.7 Conceito de elementos finitos gradados utilizando como exemplo uma variação exponencial de material. (a) Elemento Finito Homogêneo; (b) Elemento Finito Gradado (SILVA; CARBOnARI; PAULINO, 2007). . . . . . . 30

2.8 Exemplo de uma malha de elementos finitos poligonais, onde os pontos azuis são os pontos geradores. . . . . . . . . . . . . . . . . . . 31 
2.9 Procedimento para geração do diagrama de Voronoi: (a) o interior do domínio é preenchido com 10 pontos 'sementes' (círculos); (b) espelham-se os pontos auxiliares e traça-se a célula de Voronoi, para um dado ponto 'semente' (pontos auxiliares desnecessários omitidos); (c) diagrama de Voronoi completo no interior do domínio. . . . . . . . . . . . .

2.10 Num polígono convexo, todo ponto interior é um vizinho natural de todos os vértices. As dimensões geométricas $s_{i}$ e $h_{i}$ utilizadas para definir as funções de forma de Laplace estão mostradas aqui. . . . . . . . . . . . . . 34

2.11 Típica função de forma de Laplace para um hexágono regular. . . . . . . . 35

2.12 Condições de contorno para um quarto da célula unitária. . . . . . . . . . . 38

2.13 Exemplo de distribuição de material: (a) célula unitária e (b) domínio de projeto (1/4 da célula unitária); os valores de $\rho$ estão listados na Tabela 2.3. 39

2.14 Caso $1(m n=11)$ : carregamentos e funções características. . . . . . . . . . 39

2.15 Caso $2(m n=33)$ : carregamentos e funções características. . . . . . . . . . 40

2.16 Caso $3(m n=13)$ : carregamentos e funções características. . . . . . . . . . 40

2.17 Caso $4(m=1)$ : carregamentos e funções características. . . . . . . . . . . 40

2.18 Caso $5(m=3)$ : carregamentos e funções características. . . . . . . . . . 41

2.19 Ao invés de considerar as variáveis de projeto como contínuas, define-se $N N$ materiais candidatos a serem considerados na otimização. . . . . . . 41

2.20 Técnica de projeção. . . . . . . . . . . . . . . . . . . . 43

2.21 Domínio de projeto: exemplo de malha de $10 \times 10$ elementos (as variáveis de projeto são definidas para cada nó). . . . . . . . . . . . . . . . . 51

2.22 Fluxograma do algoritmo de otimização. . . . . . . . . . . . . . . . . . . . 52

2.23 Desenho esquemático do domínio de projeto em $1 / 4$ da célula unitária formada por $2 \times 2$ elementos e o mapeamento dos valores nodais das pseudo-densidades de material e das direções de polarização. . . . . . . . . . 53

2.24 (a) Modelo adotado para se calcular as tensões mecânicas macroscópicas e (b) exemplo de célula unitária do piezocompósito 2-2 . . . . . . . . . . . . 54 
2.25 Microestrutura otimizada sem gradação de material, utilizando elementos quadriláteros e direção de polarização fixada na vertical: (a) distribuição de material, (b) matriz periódica e (c) distribuição das tensões microscópicas no interior da célula unitária. . . . . . . . . . . . . . . . 55

2.26 Microestrutura otimizada sem gradação de material, utilizando elementos poligonais e direção de polarização fixada na vertical: (a) distribuição de material, (b) matriz periódica e (c) distribuição das tensões microscópicas no interior da célula unitária. . . . . . . . . . . . . . . .

2.27 Microestrutura otimizada sem gradação de material, utilizando elementos quadriláteros e direção de polarização livre: (a) distribuição de material, (b) matriz periódica e (c) distribuição das tensões microscópicas no interior da célula unitária. . . . . . . . . . . . . . . . . . .

2.28 Microestrutura otimizada sem gradação de material, utilizando elementos poligonais e direção de polarização livre: (a) distribuição de material, (b) matriz periódica e (c) distribuição das tensões microscópicas no interior da célula unitária. . . . . . . . . . . . . . . . . 5 56

2.29 Microestrutura otimizada com gradação de material, utilizando elementos quadriláteros e direção de polarização fixada na vertical: (a) distribuição de material, (b) matriz periódica e (c) distribuição das tensões microscópicas no interior da célula unitária. . . . . . . . . . . . . . . . . 5

2.30 Microestrutura otimizada com gradação de material, utilizando elementos poligonais e direção de polarização fixada na vertical: (a) distribuição de material, (b) matriz periódica e (c) distribuição das tensões microscópicas no interior da célula unitária. . . . . . . . . . . . . . . . 5

2.31 Microestrutura otimizada com gradação de material, utilizando elementos quadriláteros e direção de polarização livre: (a) distribuição de material, (b) matriz periódica e (c) distribuição das tensões microscópicas no interior da célula unitária. . . . . . . . . . . . . . . . . .

2.32 Microestrutura otimizada com gradação de material, utilizando elementos poligonais e direção de polarização livre: (a) distribuição de material, (b) matriz periódica e (c) distribuição das tensões microscópicas no interior da célula unitária.

2.33 Gráfico da função objetivo para o modelo com gradação funcional, polarização livre e malha poligonal (Fig. 2.32) . . . . . . . . . . . . . . 
3.1 Propagação de uma onda elástica em um material (a) sem band gap e (b) com band gap. . . . . . . . . . . . . . . . . . . . . . . 62

3.2 Exemplo da influência das propriedades intermediárias (regiões cinzentas) na célula unitária: (a) resultado otimizado e (b) pós-processado. . . . . . . . 64

3.3 Exemplo de aplicação do conceito MGF: desenho esquemático de um transdutor piezelétrico com um piezocompósito com gradação funcional. . . 65

3.4 Variáveis de entrada e saída do Método da Matriz Em Cadeia. . . . . . . . 68

3.5 Desenho esquemático representando um modelo de $n$ camadas, onde cada uma possui uma propriedade distinta de material. . . . . . . . . . . . .

3.6 (a) Esquemático de uma célula unitária infinitamente periódica nas direções $x$ e $y$; (b) primeira zona de Brillouin para uma célula unitária ortotrópica $\left(\Gamma \rightarrow \mathrm{X}_{1} \rightarrow \mathrm{M} \rightarrow \mathrm{X}_{2} \rightarrow \Gamma\right) \ldots \ldots \ldots \ldots$. . . . . . . . . . . .

3.7 Exemplo de problema de otimização de material fonônico unidimensional. . 74

3.8 (a) Domínio de projeto alocado em 1/8 da célula unitária e (b) célula unitária completa indicando a direção de polarização. . . . . . . . . . . . . 76

3.9 Fluxograma do problema de otimização para o projeto de materiais piezocompósitos fonônicos, tanto para o modelo unidimensional, quanto bidimensional. . . . . . . . . . . . . . . . . . 77

3.10 Modelo analítico utilizado para verificação do modelo numérico. . . . . . 78

3.11 Modelo numérico utilizado para verificação do modelo. . . . . . . . . . . . 78

3.12 Comparação dos diagramas de dispersão dos modelos analítico e numérico, considerando: (a) 5, (b) 10 e (c) 20 células unitárias. . . . . . . . . . . . . 79

3.13 Dimensões do protótipo fabricado, utilizado para verificação do modelo. 80

3.14 (a) Desenho esquemático do aparato experimental; (b) imagem do ensaio experimental. . . . . . . . . . . . . . . . . . 80

3.15 Gráfico da função transferência dos transdutores utilizados nos ensaios experimentais.

3.16 Comparação dos diagramas de dispersão do modelo analítico e medido experimentalmente: intervalos de frequência (a) de 0 a $2 \mathrm{MHz}$ e (b) de 2 a

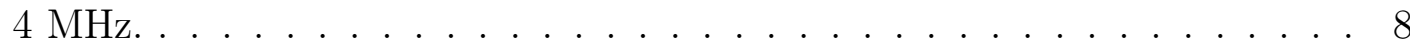


3.17 Exemplo do influência do efeito piezelétrico na célula unitária com camadas alternadas de PZT-5A e alumínio: (a) célula unitária e (b) distribuição de material. . . . . . . . . . . . . . . . . . 82

3.18 Diagramas de dispersão mostrando a influência do efeito piezelétrico na célula unitária com camadas alternadas de PZT-5A e alumínio. . . . . . . . 82

3.19 Resultado otimizado com primeiro band gap prescrito entre (a) 400 a 500 $\mathrm{kHz}$ e (b) 500 a $600 \mathrm{kHz} \ldots \ldots \ldots$. . . . . . . . . . . . . . . 84

3.20 Resultado otimizado com primeiro band gap prescrito entre (a) 600 a 700 $\mathrm{kHz}$ e (b) 700 a $800 \mathrm{kHz} \ldots \ldots \ldots$. . . . . . . . . . . . . . 84

3.21 Resultado otimizado com terceiro band gap prescrito entre (a) 1,5 a 1,6 $\mathrm{MHz}$ e (b) 1,6 a $1,7 \mathrm{kHz} \ldots \ldots \ldots$. . . . . . . . . . . . . . . 85

3.22 Verificação do modelo de elementos finitos bidimensional implementado com um modelo analítico (linhas: analítico; pontos: resultados do MEF). . 86

3.23 Verificação do modelo numérico implementado: (a) célula unitária de PVC com furo cilíndrico e (b) diagrama de dispersão (cinza: band gap). . . . . . 87

3.24 Influência do efeito piezelétrico na célula unitária com cilindros de PZT-5A inclusos em uma matriz de epóxi: (a) célula unitária e matriz periódica, (b) diagrama de dispersão sem propriedades piezelétricas e (c) com propriedades piezelétricas (amarelo: PZT-5A, azul: epóxi) . . . . . . . 88

3.25 Células unitárias, matrizes periódicas e diagramas de dispersão para a maximização do band gap entre a $3^{a}$ e $4^{a}$ frequência: (a) resultado da otimização topológica e (b) resultado interpretado (amarelo: PZT-5A, azul: epóxi) . . . . . . . . . . . . . . . . . . . . .

3.26 Células unitárias, matrizes periódicas e diagramas de dispersão para a maximização do band gap entre a $6^{a}$ e $7^{a}$ frequência: (a) resultado da otimização topológica e (b) resultado interpretado (amarelo: PZT-5A, azul: epóxi) . . . . . . . . . . . . . . . . . . . .

3.27 Células unitárias, matrizes periódicas e diagramas de dispersão para a maximização do band gap entre: (a) $7^{a}$ e $8^{a}$ frequência e (b) $10^{a}$ e $11^{a}$ frequência (amarelo: PZT-5A, azul: epóxi) . . . . . . . . . . . . . . 90

3.28 Células unitárias, matrizes periódicas e diagramas de dispersão para a maximização do band gap entre: (a) $12^{a}$ e $13^{a}$ frequência e (b) $13^{a}$ e $14^{a}$ frequência (amarelo: PZT-5A, azul: epóxi). . . . . . . . . . . . . . . 91 
3.29 Células unitárias, matrizes periódicas e diagramas de dispersão para a maximização do band gap entre: (a) $15^{a}$ e $16^{a}$ frequência e (b) $17^{a}$ e $18^{a}$ frequência (amarelo: PZT-5A, azul: epóxi). . . . . . . . . . . . . . . 91

3.30 Células unitárias, matrizes periódicas e diagramas de dispersão para a maximização do band gap entre: (a) $21^{a}$ e $22^{a}$ frequência e (b) $24^{a}$ e $25^{a}$ frequência (amarelo: PZT-5A, azul: epóxi) . . . . . . . . . . . . . 92

3.31 Pesos da projeção em função do raio de gradação $r_{g r a d}$, para diferentes valores do coeficiente de penalização $q$ (ver Fig. 2.20) . . . . . . . . . . . . 92

3.32 Células unitárias que maximizam a largura do band gap entre a $3^{a}$ e $4^{a}$ frequência, para diversos valores do coeficiente de gradação $q$ (amarelo: PZT5A; azul: epóxi). . . . . . . . . . . . . . . 93

3.33 Maximização do band gap entre a $3^{a}$ e $4^{a}$ frequência para diferentes valores de $q$ : (a-f) diagramas de dispersão; (g) Band gaps em função do coeficiente

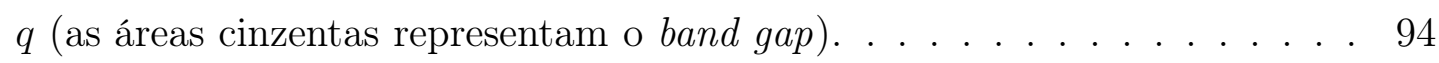

3.34 Células unitárias que maximizam a largura do band gap entre a $24^{a}$ e $25^{a}$ frequência, para diversos valores do coeficiente de gradação $q$ (amarelo: PZT5A; azul: epóxi). . . . . . . . . . . . . . . 95

3.35 Maximização do band gap entre a $24^{a}$ e $25^{a}$ frequência para diferentes valores de q: (a-f) diagramas de dispersão; (g) Band gaps em função do coeficiente $q$ (as áreas cinzentas representam o band gap) . . . . . . . . . 96

4.1 Substituição da gradação de material pela gradação geométrica, no interior da célula unitária. . . . . . . . . . . . . . . . . . . . 100

4.2 Etapas do projeto de transdutores piezelétricos utilizando o MOT. . . . . . 103

4.3 Ilustração do conceito MTOP: (a) malha de elementos finitos; (b) malha de pseudo-densidades e (c) variáveis de projeto. . . . . . . . . . . . 105

4.4 Exemplo de gradação geométrica em uma direção; as variáveis de projeto nodais primárias são indicadas em cinza, as variáveis de projeto mapeadas em preto e as repetições de padrão são indicadas pelo gradiente de cores. 105

4.5 Exemplo do filtro de projeção com raio variável. . . . . . . . . . . . . 106

4.6 Fluxograma do algoritmo de otimização. . . . . . . . . . . . . . . . . 109

4.7 Domínio de projeto adotado. . . . . . . . . . . . . . 109 
4.8 Circuito elétrico do modelo adotado (a seta indica o sentido da polarização piezelétrica). . . . . . . . . . . . . . . . . . . . 110

4.9 Caso 1: sem repetição de padrões. . . . . . . . . . . . . . . . . . . 110

4.10 Caso 2: com repetição de padrão. . . . . . . . . . . . . . . . . . . . . 110

4.11 Caso 3: com gradação geométrica. . . . . . . . . . . . . . . . . . 111

4.12 Gráfico da potência elétrica em função da frequência de excitação para diferentes mínimos locais, referente ao caso 1 (sem repetição de padrões). 112

4.13 Gráfico da potência elétrica em função da frequência de excitação para diferentes mínimos locais, referente ao caso 1 (com repetição de padrão). . 113

4.14 Gráfico da potência elétrica em função da frequência de excitação para diferentes mínimos locais, referente ao caso 1 (com gradação geométrica). 113

4.15 Melhor resultado para cada caso analisado. . . . . . . . . . . . . . . 114

4.16 Gráfico da potência elétrica gerada no resistor em função da frequência de excitação. . . . . . . . . . . . . . . . . . . . . 115

5.1 Exemplo de material onde a região piezelétrica é a menos rígida do piezocompósito. . . . . . . . . . . . . . . . . . . 120

A.1 Efeitos (a) longitudinais e (b) transversais das cerâmicas piezelétricas. . . . 138

C.1 Conceito de Multi-escala ilustrando as dimensões macro (x) e micro (y). . 148

C.2 Condições periódicas na célula unitária. . . . . . . . . . . . . . . . . . 153

D.1 (a) Exemplo de um piezocompósito 2-2; (b) Exemplo de célula unitária do piezocompósito $2-2 \ldots \ldots$. . . . . . . . . . . . . . . . . . 160

D.2 Piezocompósito 2-2: caracterização de desempenho em função da fração de volume de PZT-5A . . . . . . . . . . . . . . . . . . . . . . . 161

E.1 Conceito de domínio estendido fixo. . . . . . . . . . . . . . . . . 162

E.2 Exemplo de instabilidade de tabuleiro de xadrez. . . . . . . . . . . . . . 167 


\section{Lista de Tabelas}

2.1 Condições de contorno em $x=0$ e $x=X_{1} \ldots \ldots \ldots$. . . . . . . 37

2.2 Condições de contorno em $z=0$ e $z=Z_{3} \ldots \ldots \ldots$. . . . . . 37

2.3 Exemplo: distribuição de material em uma malha de $7 \times 7$ elementos (ver Fig. 2.13b). . . . . . . . . . . . . . . . . . . . . . 39

2.4 Propriedades dos materiais PZT-5A e polímero epóxi. . . . . . . . . . . 53

2.5 Propriedades efetivas das microestruturas otimizadas sem gradação de material. . . . . . . . . . . . . . . . . . 56 56

2.6 Comparação da microestruturas otimizadas, sem gradação de material. . 56

2.7 Propriedades efetivas das microestruturas otimizadas com gradação de material. . . . . . . . . . . . . . . . . . . . . 59

2.8 Comparação da microestruturas otimizadas, com gradação de material. . . 60

3.1 Comparação das larguras dos band gaps dos exemplos da Fig. 3.2. . . . . . 64

3.2 Tabela de propriedades do material PZT-5A e do alumínio utilizadas no modelo unidimensional. . . . . . . . . . . . . . . . . . . . . . 82

3.3 Largura dos band gaps obtidos com e sem polarização piezelétrica. . . . . . . 83

C.1 Condições de contorno em $x=0$ e $x=Y_{1} \ldots \ldots \ldots \ldots$. . . . . . 158

C.2 Condições de contorno em $z=0$ e $z=Y_{3}$. . . . . . . . . . . 158

D.1 Valores máximos das características de desempenho dos materiais PZT-5A e piezocompósito 2-2 (PZT-5A/Epóxi) . . . . . . . . . . . . . . . 160 


\section{Lista de Abreviaturas e Siglas}

CAMD Continuous Approximation of Material Distribution

MEF Método de Elementos Finitos

MEMS Microelectromechanical Systems

MGF Material com Gradação Funcional

MMA Method of Moving Asymptotes

MMC Método de Matriz Em Cadeia

MOT Método de Otimização Topológica

MPGF Material Piezocompósito com Gradação Funcional

SIMP Solid Isotropic Material with Penalization

SPS Spark Plasma Sintering

OMD Otimização de Material Discreto

OT Otimização Topológica

PWE Plane Wave Expansion

PZT-5A Titanato Zirconato de Chumbo 


\section{Introdução}

Materiais compósitos são formados pela combinação de dois ou mais materiais distintos em escala microscópica; porém, em geral o material final apresenta propriedades homogêneas em escala macroscópica (JONES, 1998). Uma das principais vantagens de se utilizar materiais compósitos é obter propriedades distintas dos próprios materiais constituintes, quando se apresentam em estado puro. As propriedades efetivas de um compósito dependem da fração de volume dos materiais constituintes e da geometria das inclusões. Exemplos de propriedades efetivas desejáveis são: rigidez, condução térmica ou elétrica, resistência à corrosão, peso específico e isolamento acústico. O termo piezocompósito é utilizado para qualquer material compósito resultante da combinação de um material piezelétrico (polímero ou cerâmica) com outros materiais piezelétricos ${ }^{1}$ ou não-piezelétricos, incluindo espaços preenchidos de ar (NEWNHAM; SKINNER; CROSS, 1978). Em geral, estas combinações resultam em novos materiais compósitos que oferecem vantagens substanciais em relação a materiais piezelétricos convencionais. Assim, em transdutores de ultrassom e sonares, por exemplo, estas vantagens são alto acoplamento eletromecânico, o qual mede a conversão de energia no piezocompósito (portanto, sua sensibilidade), e baixa impedância acústica, que auxilia na transmissão das ondas acústicas para o meio, como tecidos do corpo humano ou água (SMITH, 1989; SAFARI, 1994). O projeto de materiais piezocompósitos tem sido aplicado a sensores e transdutores (AKDOGAN; ALLAHVERDI; SAFARI, 2005), transdutores bilaminares ou bimorphs (SMITS; DALKE; COONEY, 1991; SEELEY; CHATTOPADHYAY; BREI, 1996; IM; KIM; ROH, 1998), transdutores flextensionais (ABDALLA et al., 2005), transdutores ultrassônicos (LAMBERTI et al., 2000; ANDRADE et al., 2009; LI; LISSENDEN, 2011), piezocompósitos para coleta de energia (TIEN; GOO, 2010) e transdutores piezocompósitos de casca com orientação de fibra (KIYONO; SILVA; REDDY, 2012), entre outros.

O projeto de materiais piezocompósitos permite obter propriedades específicas para cada tipo de aplicação, o que não pode ser feito com um único material. Aplicações que envolvem piezocompósitos geralmente apresentam grandes dimensões em relação a sua célula unitária, de forma que seus desempenhos dependam basicamente das propriedades

\footnotetext{
${ }^{1}$ Uma breve descrição sobre materiais piezelétricos está disponível no Apêndice A.
} 
efetivas do piezocompósito. Das atuais configurações disponíveis de piezocompósitos, a maioria das aplicações limita-se na utilização do piezocompósito 1-3, que consiste de bastões de piezocerâmicas imersos em uma matriz polimérica, como mostrado na Fig. 1.1(a), ou no piezocompósito 2-2, que consiste em camadas alternadas de piezocerâmica e polímero, ilustrado na Fig. 1.1(b). Os termos 1-3 e 2-2 referem-se à conectividade do material piezocompósito; entretanto, muitas outras conectividades também são possíveis de se obter (NEWNHAM; SKINNER; CROSS, 1978).

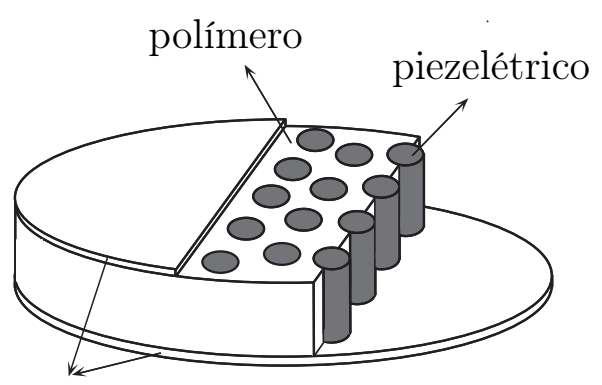

(a)

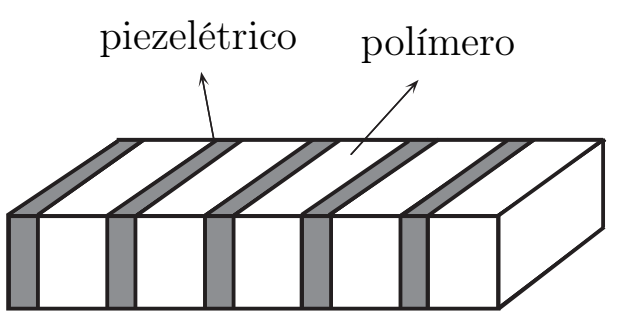

(b)

Figura 1.1: Exemplos de conectividade em materiais piezocompósitos: (a) piezocompósito 1-3 e (b) piezocompósito 2-2.

Em aplicações quasi-estáticas de piezocompósitos assume-se que os comprimentos de onda de excitação são grandes o suficiente para que a estrutura detalhada da célula unitária seja desprezível e o material possa ser considerado como um novo meio homogêneo, com novas propriedades efetivas. Assim, uma dada excitação (acústica, por exemplo) propaga-se através das pequenas variações do meio compósito, da mesma forma que se propaga na estrutura granular na escala micrométrica em uma cerâmica pura convencional (SMITH, 1989, 1993). Um comportamento homogêneo pode ser garantido reduzindo o tamanho da célula unitária (ou microestrutura) relativo ao comprimento de onda de excitação. Entretanto, em aplicações dinâmicas, isto é, quando os comprimentos de onda não são grandes o suficiente em relação ao tamanho da célula unitária, o piezocompósito apresenta um comportamento dispersivo com reflexões ocorrendo dentro da célula unitária e, portanto, há a necessidade de se utilizar abordagens diferentes do caso homogêneo.

Uma propriedade relevante dos materiais compósitos em aplicações dinâmicas é a possibilidade de ter faixas de frequência, mais conhecidas por band gaps, nas quais as ondas não se propagam. No caso das ondas elásticas os materiais são denominados por fonônicos (VASSEUR et al., 1998; TANAKA; TOMOYASU; TAMURA, 2000; SIGMUND; JENSEN, 2003), cujos exemplos de aplicações são filtros, transdutores ou criação de ambientes livres de vibrações. Já no caso das ondas eletromagnéticas, denominam-se por materiais fotônicos (COX; DOBSON, 1999; VILLENEUVE; PICHE, 1991), cujas áreas de aplicações são telecomunicações, metrologia ótica, medicina, entre outras. Nesse trabalho, decidiu-se 
focar no projeto de piezocompósitos fonônicos.

Tanto as propriedades efetivas quanto a largura dos band gaps dependem das propriedades e frações de volume dos materiais constituintes, além da topologia da distribuição destes materiais na célula unitária.

\subsection{Materiais com Gradação Funcional (MGF)}

Materiais com Gradação Funcional (MGF) são materiais compósitos cujas propriedades variam de forma contínua em uma ou mais direções. Essa variação de propriedades é, em geral, obtida através da mudança gradativa da microestrutura ${ }^{2}$ do material (ABOUDI; PINDERA; ARNOLD, 1999; KIEBACK; NEUBRAND; RIEDEL, 2003), de tal modo que não haja uma interface definida entre os materiais constituintes (REITER; DVORAK; TVERGAARD, 1997; KIEBACK; NEUBRAND; RIEDEL, 2003; YIN et al., 2007). Uma característica importante do material com gradação funcional é que a fração volumétrica das fases varia continuamente na estrutura, de forma que não haja uma interface definida entre os materiais constituintes. Em outras palavras, o material A é gradativamente substituído pelo material B, constituindo-se, assim, um material composto com a fração de material A e material B variando suavemente através de uma zona de transição (ver Fig. 1.2). Uma vantagem desse tipo de estruturas MGF é a possibilidade de se aproveitar as propriedades dos materiais base constitutivos. Por exemplo, no caso do par de materiais base metal/cerâmica, pode-se projetar uma barreira térmica que possua numa face as propriedades térmicas da cerâmica: baixa condutividade térmica e alto ponto de fusão, e na outra face as propriedades do metal: alta resistência à tração e alta resiliência. Assim, tem-se um componente que aproveita as vantagens que oferece cada material base em uma única estrutura sem interfaces entre esses materiais (KOIZUMI, 1997). Outra vantagem dos MGFs de não apresentar interface entre a microestrutura é a redução de concentração de tensões (ALMAJID; TAYA; HUDNUT, 2001; WANG; NODA, 2001; KIM; PAULINO, 2002), a qual é um problema comum em materiais compósitos. Outras vantagens são a maximização de deslocamentos em atuadores piezelétricos (CARBONARI; SILVA; PAULINO, 2009) e aumento nas forças de adesão e tempo de vida útil (QIU; ZHU; JI, 2009). Os MGFs têm sido objeto de pesquisa intensa e organizada em países tais como Estados Unidos, Japão e Alemanha. Avanços recentes no processamento de materiais têm permitido fabricar uma ampla variedade de MGFs (KIEBACK; NEUBRAND; RIEDEL, 2003). Exemplos de métodos de fabricação de MGFs são sinterização por plasma (Spark Plasma Sintering, ou SPS) e técnicas de estereolitografia, entre outros.

\footnotetext{
${ }^{2} \mathrm{O}$ termo "microestrutura" refere-se à estrutura em microescala; isso não se refere aos grãos cristalinos, interfaces entre grãos, falhas e outras características observadas com microscópio, como empregado por
} 


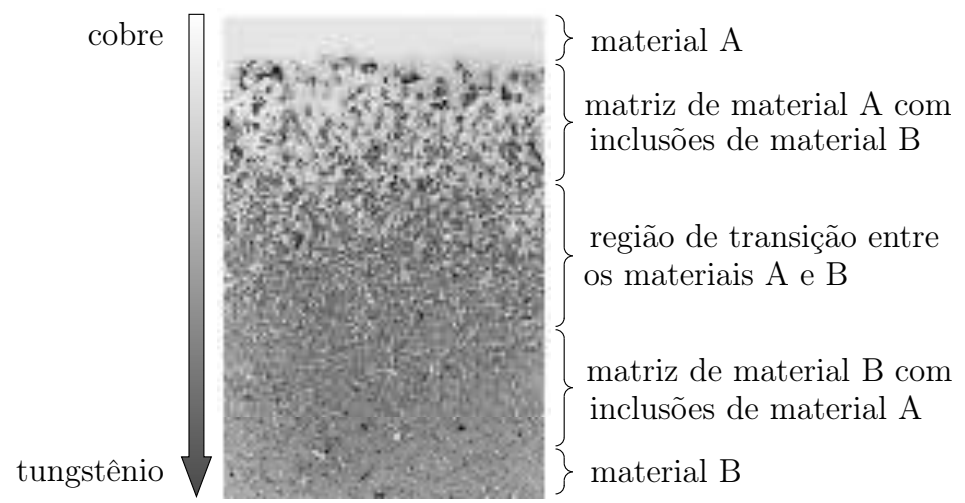

Figura 1.2: Microfotografia de um Material com Gradação Funcional (MGF)

(KIEBACK; NEUBRAND; RIEDEL, 2003).

O conceito de MGF tem sido investigado em materiais piezelétricos através de estudos analíticos (ALMAJID; TAYA, 2001; HUANG; DING; CHEN, 2007) ou numéricos (ZUH; MENG, 1995; HAUKE et al., 2000; YAS et al., 2012), bem como através do estudo de técnicas de fabricação dos mesmos (ZUH; MENG, 1995; YAMADA; OHKUBO; NAKAMURA, 2001; ALEXANDER; BREI; HALLORAN, 2005). A utilização do conceito de MGF no projeto de materiais piezocompósitos permite projetar estruturas sem interface entre os materiais constituintes (por exemplo, PZT e polímeros) reduzindo, por exemplo, o surgimento de reflexões de ondas que causam o fenômeno de espalhamento (ALMAJID; TAYA; HUDNUT, 2001) e tensões mecânicas em aplicações de potência, aumentando assim a vida à fadiga (QIU; ZHU; JI, 2009). Neste trabalho, os materiais piezocompósitos projetados utilizando o conceito de gradação funcional serão denominados de Materiais Piezocompósitos com Gradação Funcional (MPGF). A Fig. 1.3 ilustra o conceito de um MPGF.

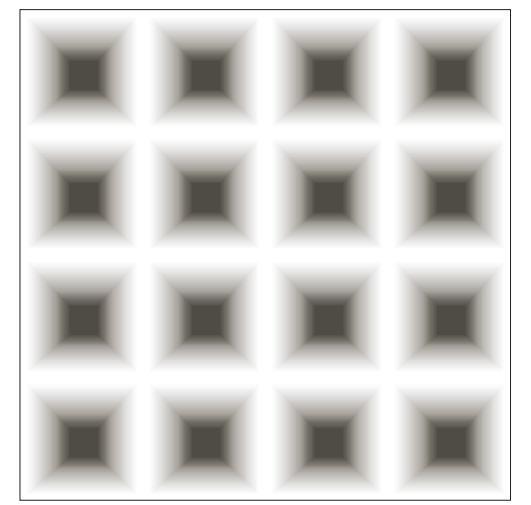

Figura 1.3: Exemplo de Material Piezocompósito com Gradação Funcional (MPGF).

\subsection{Método de Otimização Topológica (MOT)}

Otimização topológica é um método computacional de otimização estrutural que permite projetar estruturas com dois ou mais materiais (sendo que um deles pode ser um cientistas de materiais. 
vazio), distribuídos de forma ótima, de modo a extremizar (maximizar ou minimizar) uma dada função objetivo. O MOT tem sido largamente aplicado nos últimos anos no projeto da topologia de partes mecânicas com alta rigidez e para um dado peso nas indústrias automotivas e aeronáuticas (BENDSØE; SIGMUND, 2003). Esse método torna o processo de projeto mais automático, otimizado e independente da experiência específica de alguns engenheiros, fornecendo a topologia otimizada da estrutura a ser construída, atendendo aos requisitos de projeto. Obviamente, a presença do engenheiro é necessária para a obtenção da estrutura final e verificação de seu desempenho para o qual foi projetada (utilizando métodos numéricos e experimentais).

Além das aplicações na área estrutural clássica, a utilização do MOT expandiu-se para outras áreas como o projeto de mecanismos flexíveis (SIGMUND, 1997), projeto de micromecanismos com atuação térmica e eletrotérmica (SIGMUND, 2001b), projeto de atuadores flextensionais piezelétricos (SILVA; NISHIWAKI; KIKUCHI, 2000), projeto de estruturas sob atuação de campos magnéticos (YOO; KIKUCHI, 2000), projeto de materiais fonônicos e fotônicos (SIGMUND; JENSEN, 2003) entre outros.

Técnicas de otimização topológica para projetar transdutores piezelétricos e outros dispositivos tem sido amplamente utilizadas. Por exemplo, Abdalla et al. (ABDALLA et al., 2005) otimizam a distribuição de material de mecanismos flexíveis piezelétricos a fim de se maximizar a eficiência na conversão de energia, enquanto que Ha e Cho (HA; CHO, 2006) maximizam o coeficiente de acoplamento eletromecânico através da distribuição de material para ressonadores piezelétricos. Outras metodologias de projeto de atuadores piezelétricos utilizando otimização topológica são apresentadas por Carbonari et al. (CARBONARI; SILVA; NISHIWAKI, 2007; CARBONARI; SILVA; PAULINO, 2007; CARBONARI; SILVA; NISHIWAKI, 2005; CARBONARI; SILVA; PAULINO, 2009). Kang e Tong (KANG; TONG, 2008a, 2008b) utilizam otimização topológica para encontrar a distribuição de camadas piezelétricas, bem como as voltagens de acionamento para controlar o posicionamento de placas piezelétricas. Donoso e Sigmund (DONOSO; SIGMUND, 2009) utilizam otimização de forma para o dimensionamento de camadas piezelétricas em uma viga bilaminar para minimizar deflexões na ponta do atuador, tanto em aplicações estáticas quanto dinâmicas, e Donoso e Bellido (DONOSO; BELLIDO, 2009) utilizam otimização topológica para encontrar a distribuição de polarização em transdutores e sensores piezelétricos.

Nos últimos anos, diversas abordagens tem sido utilizadas para implementar o conceito do MOT, as quais podem, essencialmente, ser classificadas em dois grupos. O primeiro grupo visa a relaxação do problema de distribuição de material discreta (0-1), definindo um modelo de material que permita a distribuição contínua de material no domínio de projeto. Exemplos são métodos baseados em densidades (ROZVANY, 1992), incluindo o 
SIMP (descrito mais detalhadamente a seguir) (BENDSØE; SIGMUND, 1999), e métodos de homogeneização (BENDSøE; KIKUCHI, 1988; SUZUKI; KIKUCHI, 1991). Ao contrário, o segundo grupo utiliza uma distribuição discreta durante todo o processo de otimização e é baseado na geometria de funções de distribuição de material. Exemplos desse grupo são o método de bolhas (ESCHENAUER; KOBELEV; SCHUMACHER, 1994), derivadas topológicas (NOVOTNY et al., 2003), métodos de função implícitas, tais como "level-set" (ALLAIRE; JOUVE; TOADER, 2004; DIJK; LANGELAAR; KEULEN, 2009; WANG; WANG, 2006, 2006; CHALLIS, 2010) e "phase-field" (TAKEZAWA; NISHIWAKI; KITAMURA, 2010), métodos isogeométricos (SEO; KIM; YOUN, 2010) e métodos evolucionários (CHU et al., 1996; LI; STEVEN; XIE, 1999, 2001).

Nesse trabalho é utilizado uma abordagem baseada no conceito micro. Nessa abordagem, essencialmente o MOT procura distribuir material no interior de um domínio inicial, discretizado em elementos finitos, que permanece fixo durante o processo. Um conceito importante nessa abordagem é como variar o material entre zero e um no interior do domínio durante a otimização. O problema discreto de distribuição de material (0 ou 1), como já é conhecido, não possui solução única (SIGMUND; PETERSSON, 1998), uma vez que é observada a presença de vários mínimos locais, sendo praticamente sempre possível a determinação de um novo conjunto de parâmetros para cada discretização utilizada que melhore a função objetivo inicialmente proposta. Uma forma de se obter a solução é relaxar o problema, permitindo que o material assuma valores intermediários entre zero e um, ou seja, que possam ocorrer materiais intermediários (ou compostos) ao invés de somente material A (por exemplo, ar) ou material B (por exemplo, sólido) em cada ponto do domínio. Assim, as propriedades dos materiais intermediários, representadas pelas variáveis de projeto, variam de acordo com uma lei de "mistura" definida, chamada de modelo de material (SUZUKI; KIKUCHI, 1991; BENDSØE; KIKUCHI, 1988). Esses valores intermediários são então penalizados, de modo que a solução final obtida seja da forma discreta. Um exemplo de modelo de material comumente utilizado é o SIMP (Solid Isotropic Microstructure with Penalization) (BENDSøE; SIGMUND, 1999), que utiliza uma interpolação não-linear para as propriedades do material, no qual os valores intermediários apresentam pequenos valores de rigidez em comparação a quantidade de material empregado. Comumente nesse modelo de material emprega-se uma função exponencial do tipo $\rho(x)^{p}$, tal que $x \in \Omega$, sendo $\Omega$ o domínio no qual ocorre a distribuição de material na estrutura. No entanto, ao se fazer isso retornam-se às dificuldades do problema discreto, como por exemplo, a não-unicidade de solução, formações de tabuleiro de xadrez e dependência de malha (ver Apêndice E). Assim, por exemplo, no caso do problema de máxima rigidez para um certo volume de material, podemos obter uma solução de topologia com poucos furos grandes ou com muitos furos pequenos. Para 
contornar esses problemas, são adicionadas restrições de controle de complexidade ao problema, como por exemplo, restrições nos gradientes das variáveis de projeto ao longo do domínio através de filtros de controle de gradientes (BENDSØE; SIGMUND, 2003), filtros de projeção (BRUNS; TORTORELLI, 2001) ou restrições de perímetro (HABER; JOG; BENDSØE, 1996).

No caso do projeto de materiais compósitos, o objetivo é obter materiais com propriedades não encontradas na natureza, que são denominados de metamateriais. Nesse caso, o domínio de projeto é o domínio da célula unitária do material, como mostrado na Fig. 1.4.

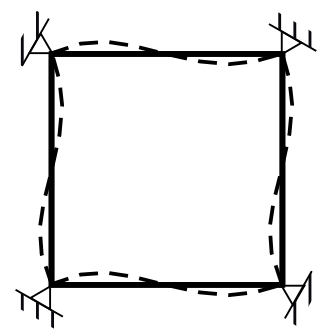

Domínio inicial

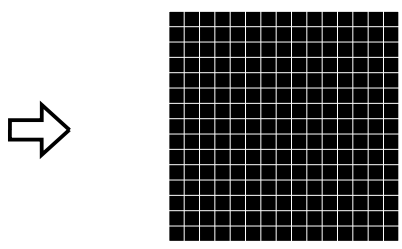

Domínio discretizado
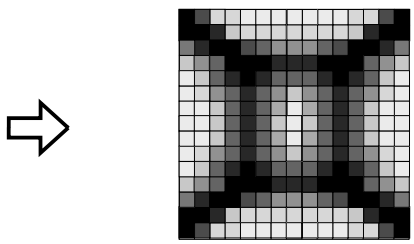

Resultado da OT
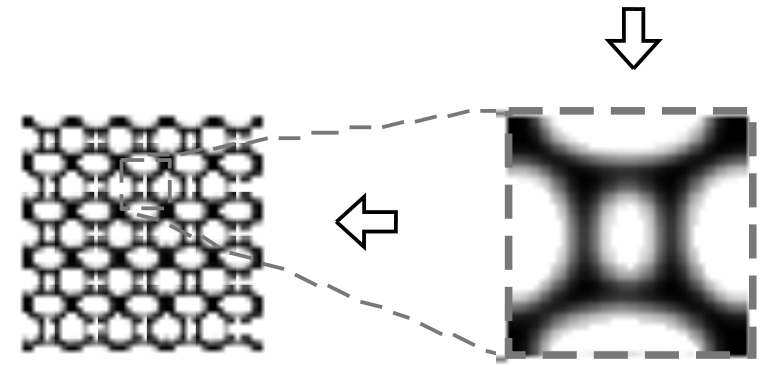

Verificação final

Pós-processamento

(matriz do material composto)

Figura 1.4: Etapas do método de otimização topológica (MOT) no projeto da célula unitária de materiais compostos.

Para encontrar a distribuição ótima o método combina, na sua implementação tradicional, algoritmos de otimização com o método de elementos finitos. A cada iteração, a distribuição de material é alterada utilizando o algoritmo de otimização e o domínio corrente é analisado pelo método de elementos finitos para o cálculo da função objetivo e restrições da otimização. A iteração prossegue até que se atinja a convergência no valor da função objetivo. Após atingida a convergência obtém-se como resultado final a distribuição de material ao longo do domínio inicial. Consiste, portanto, num problema multivariável de otimização. Sem a utilização de um algoritmo de otimização, milhões de análises seriam necessárias para encontrar a distribuição ótima de material no domínio. O MOT tem como grande vantagem atuar num domínio fixo, eliminando o problema de remalhamento da malha de elementos finitos, como ocorre no método de otimização de forma. 
Neste trabalho, primeiramente o MOT é combinado com o método de homogeneização para se projetar MPGF utilizados em aplicações quasi-estáticas. Em seguida, o MOT é aplicado no projeto de materiais piezocompósitos fonônicos com gradação funcional de materiais, utilizando-se teorias de propagação de ondas elásticas em estruturas infinitamente periódicas. Posteriormente, o MOT é aplicado no projeto de estruturas piezocompósitas utilizadas em aplicações dinâmicas em conjunto com o conceito de gradação geométrica, como por exemplo, em dispositivos para coleta de energia. Uma breve descrição de cada um destes estudos é apresentada a seguir.

\subsection{Projeto de materiais piezocompósitos com gradação funcional para aplicações quasi-estáticas}

Nesse tópico é proposta uma metodologia baseada no MOT e no método de homogeneização para se projetar MPGF, onde a aplicação adotada como exemplo é a coleta de energia. É investigada a influência das direções de polarização piezelétrica e de gradação de material entre as fases constituintes na célula unitária na conversão de energia. Geralmente é obtido um gradiente entre os materiais nos processos de manufatura dos materiais compósitos e com esse estudo pode-se avaliar sua influência no desempenho final do material. O método de homogeneização é implementado utilizando o conceito de elementos finitos gradados, o qual leva em consideração a gradação contínua no interior dos elementos finitos. A sensibilidade das propriedades homogeneizadas são calculadas utilizando o método adjunto. A maioria dos trabalhos sobre projeto de materiais adotam o elemento finito quadrilátero tradicional com quatro nós; entretanto, malhas formadas por este tipo de elemento apresentam conexões de um único nó e são suscetíveis ao problema de tabuleiro de xadrez, o qual é bem conhecido em problemas de otimização topológica. Neste aspecto, elementos poligonais podem contornar estes problemas. Assim, neste capítulo são implementados diagramas de Voronoi para gerar malhas de elementos poligonais não regulares e seus efeitos são comparados aos dos elementos quadriláteros no projeto de piezocompósitos. Ao final, são apresentados alguns exemplos bidimensionais para ilustrar esta metodologia.

As direções de polarização de materiais piezelétricos propriamente ditas podem ser definidas como variáveis de projeto no MOT. Entretanto, a desvantagem desta abordagem é que o espaço de soluções global torna-se não-convexo e, portanto, surge o problema de múltiplos mínimos locais (STEGMANN; LUND, 2005). O método denominado por Otimização de Material Discreto (OMD) utiliza gradientes combinados com programação matemática para resolver um problema discreto de otimização. Este método pode ser 
utilizado para resolver problemas de orientação de polarização de materiais piezelétricos, reduzindo o risco de se obter soluções ótimas locais. Assim, são definidos diversos tensores piezelétricos, cada um com as propriedades piezelétricas calculadas para um determinado ângulo de polarização candidato, e o método de OMD é encarregado de definir apenas um desses tensores ao final do processo de otimização.

No entanto, no projeto de materiais piezocompósitos os valores de tensões calculados considerando o material homogêneo com propriedades efetivas apenas informam o comportamento do material do ponto de vista macroscópico, ignorando assim os efeitos causados no interior da célula unitária. Assim, é necessário calcular a distribuição de tensões no interior da célula unitária na etapa de projeto do material. Estas informações são particularmente úteis para se determinar possíveis pontos de falha do material. Guedes e Kikuchi (GUEDES; KIKUCHI, 1990) utilizaram o método de homogeneização para calcular as distribuições de tensão e deformação em estruturas compósitas. Ni et al. (NI; ZHU; WANG, 2006) investigaram as tensões mecânicas em um compósito composto por reforços esféricos e outro formado por reforços de fibras unidirecionais, calculando os valores microscópicos das tensões. Uma das conclusões foi que os valores efetivos das tensões mecânicas calculados em um modelo macroscópico podem ser menores do que os máximos valores reais que ocorrem na microestrutura. Nesse estudo são calculados os valores macroe microscópicos de tensões mecânicas utilizando o método de homogeneização quando os materiais piezocompósitos são submetidos a esforços externos.

\subsection{Projeto de materiais piezocompósitos fonônicos}

O método de homogeneização discutido anteriormente pode ser utilizado apenas para aplicações estáticas, isto é, quando o comprimento de onda do fenômeno de interesse é muito maior do que o tamanho da célula unitária. Entretanto, o interesse neste trabalho é investigar a influência da gradação de material também no projeto de materiais piezocompósitos para aplicações dinâmicas. Assim, nesse estudo é apresentada uma metodologia sistemática e genérica para o projeto de materiais piezocompósitos fonônicos com gradação funcional. Para isso, duas abordagens são investigadas: a primeira, de modelagem unidimensional, visa projetar materiais piezocompósitos fonônicos com posição de band gaps prescrita, utilizando o Método de Matriz Em Cadeia (MMC) em conjunto ao MOT. Esse estudo tem a finalidade de se entender a física do problema de propagação de ondas elásticas em materiais compósitos. A segunda abordagem, de modelagem bidimensional, visa o projeto de piezocompósitos com e sem gradação funcional, com o objetivo de maximizar band gaps de diversas ordens, utilizando o MOT combinado ao MEF. Nesta abordagem também é investigada a influência de diversas 
curvas de gradação de material nos materiais piezocompósitos fonônicos. Os resultados e discussões são apresentados no Capítulo 3.

Considerando operações dinâmicas, as aplicações exigem o projeto de materiais compósitos com band gaps maximizados ou, em alguns casos, com posição prescrita. Diversos trabalhos tem sido realizados focando o aumento da largura dos band gaps (SIGMUND; JENSEN, 2003; ZHANG et al., 2003; WU et al., 2009; WEI et al., 2009) e uma das conclusões é que essa largura é determinada pelo contraste das propriedades elásticas dos materiais constituintes, assim como pela fração de volume destes na célula unitária da matriz periódica. Grande parte dos trabalhos publicados até o momento concentram-se em materiais fonônicos formados por apenas materiais isotrópicos (KEE et al., 2000; GOFFAUX; VIGNERON, 2001; LAI; ZHANG, 2003; ZHANG et al., 2003). Entretanto, materiais anisotrópicos podem influenciar o comportamento das ondas em função das direções de propagação. Hou et al. (HOU; FU; LIU, 2003) investigaram o comportamento dispersivo de um cristal fonônico bidimensional composto por uma matriz isotrópica com inclusões anisotrópicas, concluindo que a largura dos band gaps pode ser aumentada alterando-se direção dos elementos anisotrópicos. Para o caso de materiais piezelétricos, o fenômeno da dispersão das ondas é mais complexo, pois não somente a anisotropia mas também o efeito da piezeletricidade influenciam na propagação das ondas. Por exemplo, a velocidade de propagação de ondas elásticas em materiais piezelétricos pode ser aumentada pelo efeito piezelétrico (AULD, 1990).

\subsection{Projeto de estruturas piezocompósitas com gradação geométrica}

Conforme descrito anteriormente, uma das propostas desse estudo é expandir o conceito de gradação funcional para o conceito de gradação geométrica. Embora haja diversos métodos de fabricação que viabilizem a manufatura de estruturas MGF, ainda existem dificuldades que limitam o uso de MGF em larga escala. Este conceito expandido de gradação tem o objetivo de explorar as vantagens de se obter uma estrutura cujas propriedades variem de forma funcional ao longo dos seus comprimentos, porém com uma distribuição de material discreta, contornando assim possíveis dificuldades de manufatura.

Além disso, mesmo no MGF, quando observados em uma escala suficientemente pequena, é possível notar que a distribuição de materiais na microestrutura assume uma forma discreta. Por exemplo, a região de transição entre os materiais A e B da Fig. 1.2, no sentido do material A para o B, é formada inicialmente por inclusões de material B na matriz de material A. Essas inclusões de material B aumentam gradativamente em 
quantidade e tamanho, até que a condição se inverta, isto é, até que essa região seja constituída de inclusões de material A na matriz de material B. Essa mudança gradativa na estrutura do material pode ser interpretada conforme mostra a Fig. 1.5, onde é possível ver a evolução da inclusão ao longo do material.

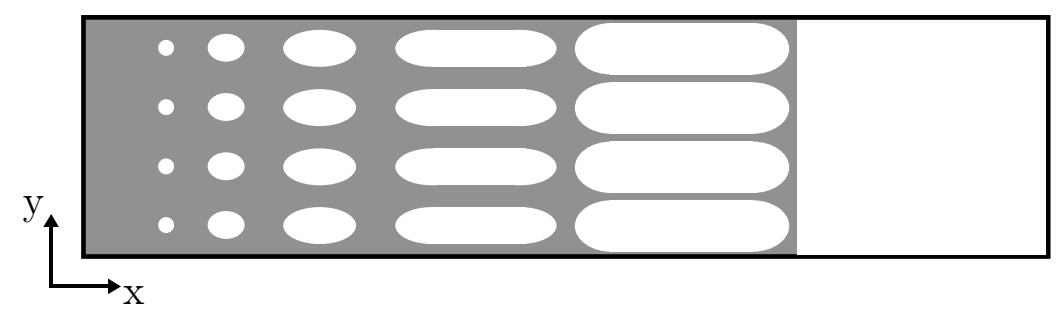

Figura 1.5: Exemplo de gradação geométrica.

Assim, nesse estudo investiga-se o conceito expandido de gradação funcional de material, utilizando o conceito de gradação geométrica baseado em repetições de padrão ao longo do domínio de projeto. Cada repetição tem um ou mais comprimentos modificados, de forma gradual. Dessa forma, embora a distribuição de materiais seja discreta, suas propriedades (por exemplo, rigidez) se alteram progressivamente ao longo da estrutura. A repetição de padrões é definida como o número de vezes que uma determinada região pré-definida, denominada padrão, de uma estrutura qualquer é repetida ao longo de uma ou mais direções. A gradação geométrica consiste em alterar continuamente (ou gradativamente) uma ou mais dimensões do padrão a cada repetição ao longo da estrutura (ver Fig. 4.1).

\section{Gradação Funcional de Material}

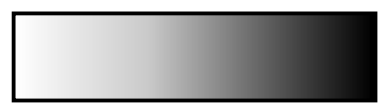

célula unitária domínio de projeto

\section{Gradação Funcional Geométrica}

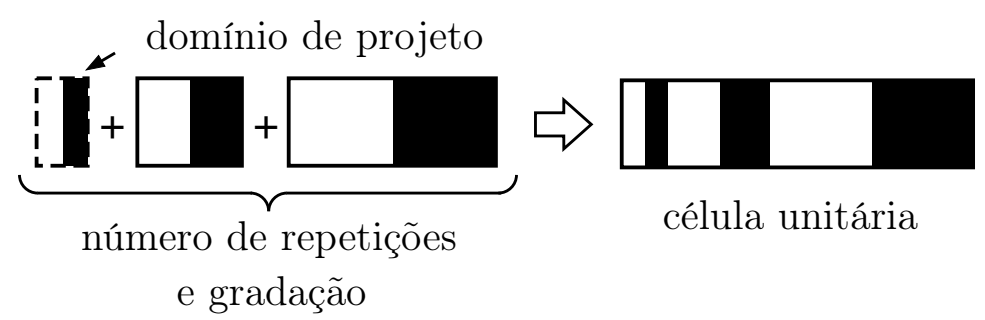

Figura 1.6: Substituição da gradação de material pela gradação geométrica, no interior da célula unitária.

Enquanto que o estudo apresentado no Capítulo 2 limita-se a aplicações quasi-estáticas de microestruturas piezocompósitas na coleta de energia segundo uma abordagem de microescala, nesse tópico é investigado a aplicação do conceito de gradação geométrica (STROMBERG et al., 2011) no projeto de estruturas piezocompósitas considerando a mesma aplicação, porém em aplicações dinâmicas, segundo uma abordagem de macroescala.

O MOT é utilizado para otimizar a distribuição de materiais no domínio de projeto a fim de maximizar uma determinada função objetivo. Novos parâmetros de projeto são adicionados, do ponto de vista da gradação geométrica, tais como o número de repetições 
do padrão no domínio de projeto e a variação das dimensões (ou gradação) do padrão ao longo da estrutura. A gradação geométrica pode ser aplicada em uma ou mais direções. A Fig. 1.7 ilustra dois casos de gradação geométrica com diferentes direções de gradação. gradação geométrica na direção $x$

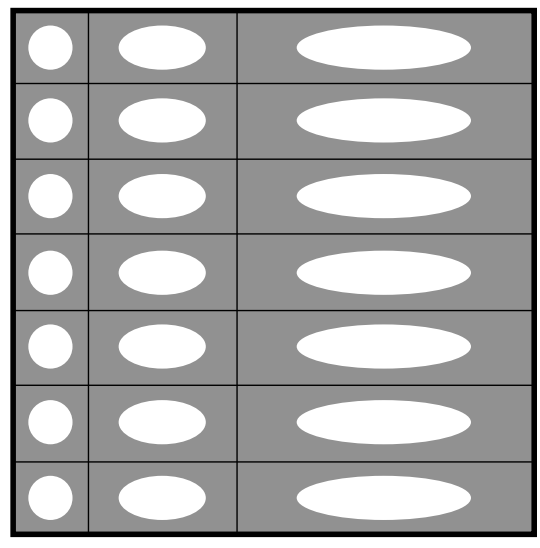

(a)

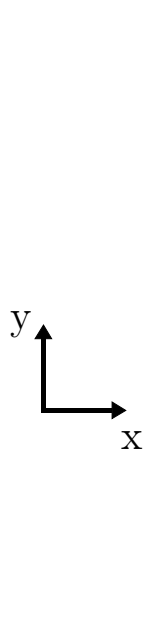
gradação geométrica nas direções $x$ e $y$

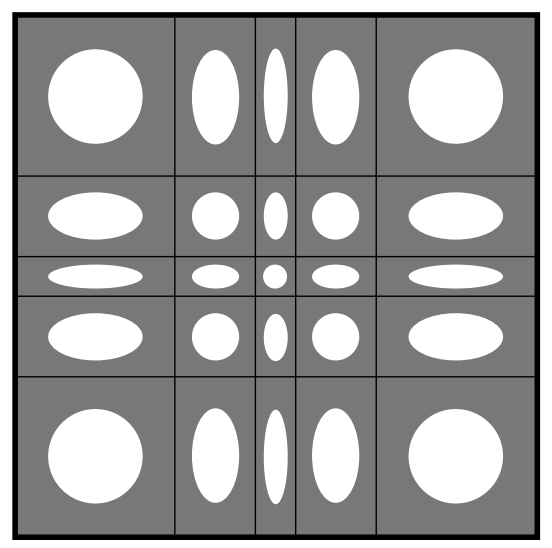

(b)

Figura 1.7: Exemplos de gradação geométrica.

O conceito de gradação geométrica pode ser aplicado tanto na microestrutura de materiais, isto é, no interior da célula unitária de uma matriz periódica (com a condição de periodicidade infinita), como em estruturas macroscópicas (sem a condição de periodicidade infinita).

\subsection{Objetivos}

Esse trabalho propõe uma metodologia para o projeto de MPGF, tanto para aplicações quasi-estáticas, quanto para aplicações dinâmicas. Dessa forma, divide-se o projeto de materiais piezocompósitos em três grupos.

O primeiro grupo consiste em um método de projeto de materiais baseado na combinação do método de homogeneização com o método de otimização topológica para o projeto de MPGF para aplicações quasi-estáticas, onde o objetivo é projetar materiais piezocompósitos que, de modo geral, maximizem a conversão de energia mecânica em elétrica. A aplicação utilizada como exemplo neste trabalho são materiais empregados em dispositivos de coleta de energia.

O segundo grupo visa aplicações dinâmicas de materiais piezocompósitos fonônicos. Neste estudo visa-se o desenvolvimento de uma metodologia sistemática e genérica para o projeto de MPGF fonônicos com largura e posição de band gaps prescritos, empregando estruturas unidimensionais, e a maximização de band gaps de diversas ordens, empregando estruturas bidimensionais. Também é investigada a influência de diversas curvas de 
gradação de material nos materiais piezocompósitos fonônicos.

O terceiro grupo explora o conceito de gradação geométrica (STROMBERG et al., 2011) em aplicações dinâmicas. Novos parâmetros são adicionados ao projeto, tais como o número de repetições de padrão no domínio de projeto e a variação das dimensões do padrão ao longo da célula unitária. Esta abordagem é empregada visando a aplicação na coleta de energia, onde se procura maximizar a potência elétrica gerada em um resistor acoplado aos eletrodos, através da obtenção da topologia otimizada de estruturas piezocompósitas.

\subsection{Justificativa}

A utilização do conceito de MGF no projeto de materiais piezocompósitos traz grandes benefícios. Um deles é a eliminação de interfaces que dão origem a concentração de tensões e, no caso dinâmico, diminuição na reflexão de ondas no interior do material composto que causam o fenômeno de espalhamento. Outro exemplo é que métodos de fabricação desses metamateriais compostos, projetados pela técnica de projeto de materiais, tem mais facilidade em gerar uma interface com gradação do que uma interface perfeita.

O projeto de materiais piezocompósitos para aplicações quasiestáticas foi iniciado na literatura (SILVA; FONSECA; KIKUCHI, 1997; SILVA et al., 1999, 1999). Este trabalho de doutorado dá continuidade neste assunto, incluindo o conceito de gradação funcional e explorando novas técnicas, tais como elementos finitos poligonais, direção de polarização do material piezelétrico e técnica de controle de gradação. Os exemplos apresentados neste estudo focam em aplicações de coleta de energia dessas estruturas piezocompósitas, onde a otimização é crítica.

Materiais que apresentam band gaps são de grande interesse para diversas aplicações, tais como transdutores, filtros acústicos, controle de ruído, vibração, etc. Diversos trabalhos tem investigado a utilização de materiais isotrópicos em aplicações fonônicas. Já os materiais piezelétricos, que além de possuir propriedades elásticas ortotrópicas também possuem propriedades piezelétricas que podem influenciar no efeito dispersivo na propagação de ondas acústicas. Assim, o projeto de MPGF fonônicos se resume em encontrar a melhor distribuição de materiais no interior da célula unitária, de forma que os band gaps tenham uma largura e posição pré-definida ou que sejam maximizados.

Embora haja diversos métodos de fabricação que viabilizem a manufatura de estruturas MGF, ainda existem dificuldades que limitam o uso de MGF em larga escala. Esse problema pode ser contornado baseando-se o projeto de tais estruturas utilizando o 
conceito de gradação geométrica.

\subsection{Contribuições Científicas}

O desenvolvimento desse projeto tem como principal contribuição científica o projeto de materiais piezocompósitos com gradação funcional, isto é, materiais piezocompósitos inovadores e com desempenhos otimizados em aplicações quasi-estáticas e dinâmicas. Assim, as contribuições científicas principais são:

- Projeto de MPGF utilizando o MOT e o método de homogeneização incluindo: direções de polarização piezelétrica variáveis no domínio de projeto, influência da gradação de materiais constituintes, análise de concentração de tensões mecânicas no interior da célula unitária e comparação dos resultados obtidos com malhas quadriláteras ou poligonais.

- Projeto de MPGF unidimensionais com prescrição de largura e posição de band gaps;

- Projeto de MPGF bidimensionais considerando pelo MOT visando a maximização dos band gaps;

- Projeto de estruturas piezocompósitas dinâmicas com gradação geométrica utilizadas para coleta de energia, visando a maximização da potência elétrica gerada na carga (resistor).

As contribuições científicas específicas são:

- Implementação do método de homogeneização baseado no conceito de elementos finitos gradados;

- Implementação de elementos gradados poligonais piezelétricos;

- Cálculo da sensibilidade das propriedades homogeneizadas para materiais piezelétricos utilizando o método adjunto;

- Adição da direção de polarização no projeto de materiais piezelétricos utilizando a abordagem OMD;

- Avaliação de tensões mecânicas microscópicas no interior das células unitárias em materiais piezocompósitos;

- Comparação entre elementos finitos quadriláteros e poligonais no projeto de MPGF para aplicações quasi-estáticas;

- Implementação do MMC para solução analítica de materiais fonônicos piezelétricos unidimensionais;

- Maximização de band gaps de alta ordem em MPGFs. 


\subsection{Organização do Documento}

Este documento é organizado da seguinte forma: no capítulo 2 é descrito o projeto de MPGF utilizando o MOT e o método de homogeneização para aplicações quasi-estáticas, considerando as direções de polarização locais na otimização. No capítulo 3 é mostrado o projeto de MPGF para aplicações dinâmicas, utilizando-se inicialmente um modelo analítico unidimensional, a fim de se entender a física do problema de propagação de ondas elásticas em materiais compósitos. Esse projeto é estendido para modelos bidimensionais, visando a maximização de band gaps de alta ordem. No capítulo 4 é descrito o projeto de transdutores piezelétricos em operação dinâmica, explorando a gradação geométrica ao longo da estrutura. Os exemplos apresentados visam a maximização da conversão de energia mecânica em elétrica em dispositivos de coleta de energia. No capítulo 5 são listadas as conclusões obtidas neste trabalho, assim como sugestões de trabalhos futuros. No apêndice A é apresentado uma breve introdução sobre materiais piezelétricos para melhor entendimento do texto. O apêndice B descreve como é realizada a implementação numérica dos materiais piezelétricos. O apêndice C apresenta um texto complementar sobre o desenvolvimento matemático do método de homogeneização e o apêndice D mostra a verificação numérica da implementação deste método. O Apêndice E descreve brevemente o MOT e discute os problemas mais comuns encontrados durante sua implementação. 


\section{Projeto de Materiais Piezocompósitos com Gradação Funcional para Aplicações Quasi-Estáticas}

Conforme dito anteriormente, a definição utilizada neste trabalho de aplicações quasi-estáticas de piezocompósitos baseia-se que os comprimentos de onda de excitação são grandes o suficiente para que a estrutura detalhada da célula unitária seja desprezível e o material possa ser considerado como um novo meio homogêneo, com novas propriedades efetivas. Assim, um comportamento homogêneo pode ser garantido reduzindo o tamanho da célula unitária relativo ao comprimento de onda de excitação.

Na otimização de um piezocompósito o objetivo é obter um aumento nas suas características de desempenho, geralmente alterando as frações de volume dos materiais constituintes, suas propriedades, forma de inclusões e as propriedades mecânicas da matriz polimérica na célula unitária do compósito. Entretanto, o projeto do material compósito em si é uma tarefa complexa e quando as propriedades dos seus materiais constituintes variam gradualmente na célula unitária o projeto torna-se ainda mais complexo. Portanto, é necessário utilizar um método robusto e genérico no projeto de materiais compósitos, tal como o MOT.

O MOT tem sido combinado com o método da homogeneização para alterar a topologia da célula unitária, permitindo o projeto de células unitárias com formas complexas. Assim, pode-se obter uma nova classe de materiais compósitos, diferenciando-se dos tradicionais compósitos baseados em fibras reforçadas ou camadas laminadas. Estes novos materiais, denominados por metamateriais, podem ter propriedades não-usuais, tais como coeficiente de Poisson negativo (fig. 2.1a) (SIGMUND, 2001b, 2001a) ou coeficiente térmico de expansão nulo (fig. 2.1b) (SIGMUND; TORQUATO, 1997).

No processo de projeto de materiais compósitos (ou metamateriais) com propriedades 


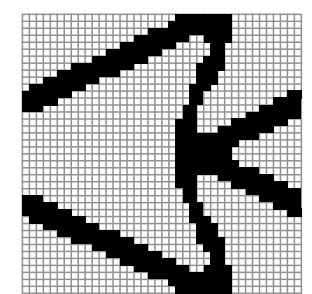

Resultado da OT

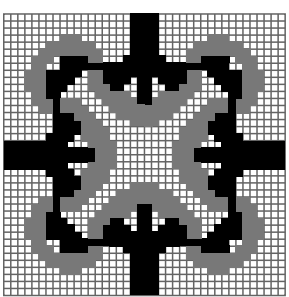

Resultado da OT

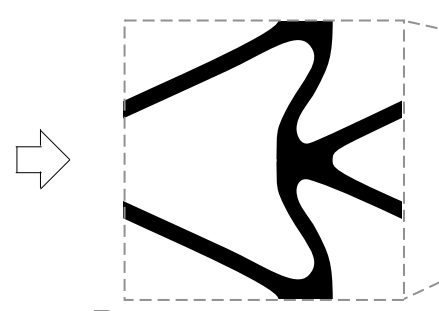

Pós-processamento

(a)

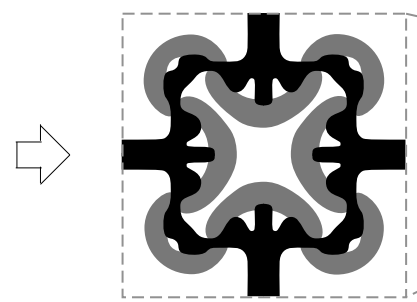

Pós-processamento

(b)

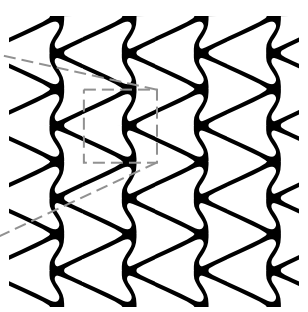

Matriz do material

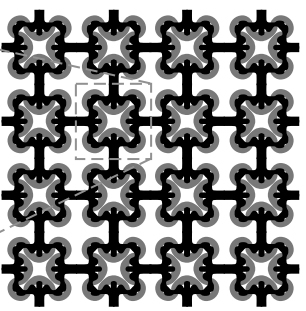

Matriz do material

Figura 2.1: Exemplo de material com (a) coeficiente de Poisson negativo; (b) coeficiente térmico de expansão nulo (SIGMUND, 2001b, 2001a; SIGMUND; TORQUATO, 1997).

melhoradas e prescritas, surge uma questão natural relacionada com as propriedades atingíveis (ou possíveis) nesse processo. Baseado no fato que a matriz constitutiva de elasticidade deve ser positiva-definida para materiais elásticos, Milton e Cherkaev (MILTON; CHERKAEV, 1995) mostraram a existência de materiais para todos os conjuntos termodinâmicos admissíveis dividindo em camadas e combinando materiais infinitamente rígidos com ar (infinitamente flexível). Porém, a condição extrema desse conjunto admissível não pode ser obtida na prática, como por exemplo, um material isotrópico com razão de Poisson igual a -1 , uma vez que um material infinitamente rígido não existe.

Outros limites foram derivados no passado. Considerando materiais com propriedades finitas, podemos citar por exemplo, o trabalho de Hashin e Shtrikman (HASHIN; SHTRIKMAN, 1963) que definiram limites para uma mistura isotrópica de materiais clássicos utilizando análise de energia, entre outros (LIPTON; NORTHRUP, 1994; GIBIANSKY; TORQUATO, 1995). Propriedades atingíveis para materiais piezelétricos foram discutidas por Smith (SMITH, 1992) considerando a condição de positivo-definido do tensor envolvendo propriedades elásticas, piezelétricas e dielétricas; entretanto, os limites não foram obtidos. Gibiansky e Torquato (GIBIANSKY; TORQUATO, 1999) também discutiram limites ótimos para compósitos laminados de matriz piezelétrica. No entanto, as propriedades extremas que podem ser obtidas pelo projeto de materiais compostos é limitada, e tem sido exploradas principalmente pela elasticidade e condutividade (LARSEN; SIGMUND; BOUWSTRA, 1997; GIBIANSKY; SIGMUND, 2000; SIGMUND, 2000a).

A partir das hipóteses que a célula unitária seja periódica e que a macroescala do 
material compósito seja muito maior do que as dimensões da microestrutura (GUEDES; KIKUCHI, 1990; SANCHEZ-PALENCIA, 1980), foram propostos diversos modelos analíticos para o cálculo de propriedades efetivas de materiais piezocompósitos. A teoria de homogeneização para materiais piezelétricos para casos quasi-estáticos (isto é, onde o comprimento de onda de excitação é muito maior do que as dimensões da célula unitária da microestrutura) foi desenvolvida por Telega (TELEGA, 1990) utilizando o método variacional de convergência- $\Gamma$. As propriedades efetivas (ou homogeneizadas) de materiais piezocompósitos periódicos complexos podem ser determinadas utilizando o método de homogeneização a partir da topologia de suas células unitárias e das propriedades dos materiais constituintes.

No projeto de compósitos tradicionais, como materiais laminados ou reforçados com fibras ou esferas, a mudança das propriedades é obtida modificando-se a localização, orientação, materiais constituintes, ou a fração em volume da fibra, esfera, ou inclusão laminar, respectivamente. Isto permite algum controle das propriedades do material compósito. Uma abordagem mais sistemática para projetar esses metamateriais tem sido desenvolvida nos últimos anos, que combina otimização topológica (BENDSØE; SIGMUND, 2003) com o método de homogeneização (GUEDES; KIKUCHI, 1990).

No campo dos materiais piezelétricos, Silva et al. (SILVA; FONSECA; KIKUCHI, 1997; SILVA et al., 1999) aplicaram o método de homogeneização combinado com técnicas de otimização topológica para projetar microestruturas de piezocompósitos com altas características de desempenho. A topologia da microestrutura foi obtida procurando a distribuição ótima de duas fases (material e vazios) na célula unitária compósita (domínio fixo). Os materiais piezocompósitos foram fabricados utilizando uma técnica de coextrusão termoplástica, que permitiu a fabricação da matriz de células unitárias onde cada célula pode atingir dimensões de até $80 \times 80 \mu \mathrm{m}$ (ver Fig. 2.2) (CRUMM; HALLORAN, 1998). Gibiansky e Torquato (GIBIANSKY; TORQUATO, 1997a, 1997b) aplicaram uma abordagem de otimização para encontrar as propriedades ótimas de um material matricial polimérico que maximizasse as características de desempenho do piezocompósito 1-3. As células unitárias desse material matricial polimérico foram projetadas posteriormente por Sigmund et al. (SIGMUND; TORQUATO, 1997), combinando o trabalho de Gibiansky e Torquato com otimização topológica e abordagens de homogeneização. Agbossou et al. (AGBOSSOU; VIET; PASTOR, 1999) utilizou o método de homogeneização para calcular as propriedades efetivas de compósitos com fibras piezelétricas unidirecionais, transdutores piezelétricos 1-3 e músculos, considerados como "materiais semi-piezelétricos". Buehler et al. (BUEHLER; BETTIG; PARKER, 2004) aplicaram o método de homogeneização para calcular as propriedades efetivas de uma célula unitária composta por um material convencional e cerâmica piezelétrica. Esta célula unitária foi utilizada em 
um problema de otimização topológica visando a maximização de deslocamento de um ponto arbitrário devido a um campo elétrico aplicado em sua estrutura. Lenglet et al. (LENGLET; HLADKY-HENNION; DEBUS, 2003) implementaram o método de elemento de volume representativo para avaliar as propriedades efetivas de piezocompósitos. Challagullaa e Venkatesh (CHALLAGULLA; VENKATESH, 2009) implementaram um modelo numérico baseado no método de homogeneização para calcular as propriedades efetivas de um piezocompósito 2-2. Trindade e Benjeddou (TRINDADE; BENJEDDOU, 2011) utilizaram o método de homogeneização para caracterizar as propriedades efetivas de um piezocompósito constituído de sete camadas (Kapton, acrílico, eletrodo, fibras piezelétricas em uma matriz de epóxi,eletrodo, acrílico e Kapton). Outros estudos tem explorado a influência da polarização de materiais piezelétricos nas propriedades efetivas, utilizando métodos probabilísticos (JAYACHANDRAN; GUEDES; RODRIGUES, 2007, 2008). Além disso, técnicas de manufatura também tem sido estudadas para construir tais metamateriais (HOY et al., 1998; CRUMM; HALLORAN, 1998; MAZUMDER; SCHIFFERER; CHOI, 1999; QI; KIKUCHI; MAZUMDER, 2004; QI; HALLORAN, 2004; REILLY; HALLORAN; SILVA, 2007; CRUMM et al., 2007; REILLY et al., 2007). No entanto, técnicas de manufatura tem sido pouco exploradas quando uma gradação de material é considerada dentro da célula unitária (MAZUMDER et al., 2000; QI; KIKUCHI; MAZUMDER, 2004).

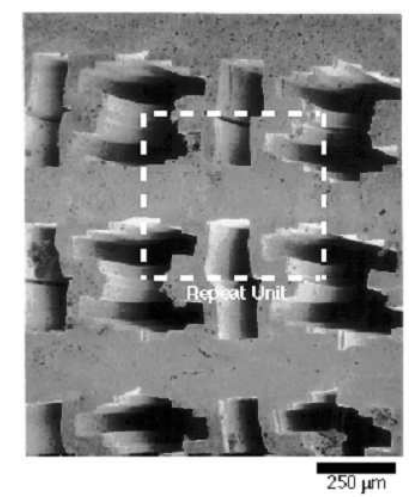

Figura 2.2: Imagem da matriz de células unitárias (WING; HALLORAN, 2006).

Nesse capítulo pretende-se explorar o projeto computacional de microestruturas de materiais piezocompósitos com gradação funcional, juntamente com a inclusão das direções de polarização locais na otimização, além de calcular os valores das microtensões no interior de cada elemento da célula unitária (VATANABE; PAULINO; SILVA, 2010a). Para isso, são implementados elementos poligonais e seu desempenho é comparado ao do elemento quadrilátero tradicional (VATANABE; PAULINO; SILVA, 2010b, 2012). Os exemplos mostrados neste capítulo são obtidos através da função objetivo de maximização da conversão de energia mecânica em elétrica em aplicações de coleta de energia. A topologia da microestrutura é obtida distribuindo-se dois materiais no domínio da célula unitária. O modelo de material adotado assume que as propriedades em cada ponto do 
domínio da célula pode variar de uma fase a outra proporcionalmente a um parâmetro $\rho$, que é a variável de projeto do problema de otimização. Para o cálculo das propriedades efetivas das microestruturas projetadas é utilizado o método de homogeneização para materiais piezelétricos MGF baseado na técnica de elementos finitos gradados (KIM; PAULINO, 2002; SILVA; CARBONARI; PAULINO, 2007), que permite levar em conta a gradação das propriedades no interior da célula unitária. Uma técnica de controle de gradiente do material na célula unitária é implementada baseada na projeção de (BRUNS; TORTORELLI, 2001). O controle de gradiente permite que se leve em conta a influência da gradação MGF no projeto de materiais, bem como, evita o problema de dependência de malha na implementação de otimização topológica (BENDSøE; SIGMUND, 2003). O estudo apresentado neste trabalho é limitado a materiais piezocompósitos formados por células unitárias bidimensionais.

Este capítulo é organizado da seguinte forma: na Seção 2.1, apresenta-se brevemente o método de homogeneização e o cálculo das tensões mecânicas no interior da célula unitária. Na Seção 2.2, é descrita a formulação do problema de otimização. Na Seção 2.3, é descrita a implementação numérica dos conceitos utilizados neste capítulo. Na Seção 2.4, são apresentados os resultados das microestruturas otimizadas. Na Seção 2.5, apresentam-se as conclusões obtidas neste capítulo.

\subsection{Fundamentação Teórica}

Nesta seção, são mostradas apenas as equações finais do método de homogeneização. As equações constitutivas piezelétricas estão brevemente descritas no Apêndice A. O desenvolvimento das equações homogêneas para a piezeletricidade utilizando o método de expansão assintótica (considerando somente os termos de primeira ordem) (SANCHEZ-PALENCIA, 1980) está descrito no Apêndice C, assim como as condições de simetria utilizadas neste capítulo.

\subsubsection{Método de Homogeneização}

O método de homogeneização computacional permite o cálculo das propriedades efetivas de materiais compósitos periódicos complexos conhecida a topologia de sua célula unitária. Trata-se de um método genérico para o cálculo de propriedades efetivas e não tem limitação de fração de volume ou forma dos materiais constituintes do compósito na célula unitária. As principais hipóteses são que a célula unitária é periódica e que a escala da peça de material composto é muito maior do que as dimensões da microestrutura (GUEDES; KIKUCHI, 1990; SANCHEZ-PALENCIA, 1980). 
A teoria de homogeneização aplicada à piezeletricidade para o caso estático (onde o comprimento de onda operacional $\gamma$ é muito maior do que as dimensões da célula unitária) foi desenvolvida por Telega (TELEGA, 1990) utilizando o método variacional de convergência- Г. Galka et al. (GALKA; TELEGA; WOJNAR, 1992) apresentou as equações homogeneizadas e propriedades efetivas referentes à termopiezeletricidade utilizando o método de expansão assintótica de dupla-escala. Para aplicações dinâmicas, onde o comprimento de onda é da mesma dimensão ou menor do que as dimensões da célula unitária, Turbé e Maugin (TURBE; MAUGIN, 1991) utilizaram a teoria de expansão de Bloch para obter as propriedades efetivas dinâmicas de um meio piezelétrico. Para baixas frequências, isto é, no limite do caso estático, as propriedades efetivas são as mesmas derivadas por Telega (TELEGA, 1990). Finalmente, Otero et al. (OTERO; CASTILlERO; RAMOS, 1997) utilizou o método de média assintótica para desenvolver equações homogeneizadas e propriedades efetivas para um meio piezelétrico (heterogêneo e periódico) considerando termos de ordem infinita na expansão assintótica. A implementação numérica do método de homogeneização para piezeletricidade (SILVA et al., 1999; SILVA; FONSECA; KIKUCHI, 1997; SILVA et al., 1999) é descrita no Apêndice C. Nesta seção, são brevemente derivadas as equações homogêneas para a piezeletricidade utilizando o método de expansão assintótica (considerando somente os termos de primeira ordem) (SANCHEZ-PALENCIA, 1980).

Considerando o procedimento padrão de homogeneização, a célula unitária é definida como $Y=\left[0, Y_{1}\right] \times\left[0, Y_{2}\right]$, as funções do material como $c_{i j k l}^{E}, e_{i j k}$ e $\varepsilon_{i j}^{S}$ são consideradas funções $Y$-periódicas:

$$
\begin{aligned}
& \mathbf{c}^{E \varepsilon}(\mathbf{x})=\mathbf{c}^{E}(\mathbf{x}, \mathbf{y}) ; \quad \mathbf{e}^{\varepsilon}(\mathbf{x})=\mathbf{e}(\mathbf{x}, \mathbf{y}) ; \quad \varepsilon^{S \varepsilon}(\mathbf{x})=\varepsilon^{S}(\mathbf{x}, \mathbf{y}) \\
& \mathbf{c}^{E \varepsilon}(\mathbf{x}, \mathbf{y})=\mathbf{c}^{E}(\mathbf{x}, \mathbf{y}+Y) ; \quad \mathbf{e}^{\varepsilon}(\mathbf{x}, \mathbf{y})=\mathbf{e}(\mathbf{x}, \mathbf{y}+Y) ; \quad \varepsilon^{S \varepsilon}(\mathbf{x}, \mathbf{y})=\varepsilon^{S}(\mathbf{x}, \mathbf{y}+Y)
\end{aligned}
$$

e $\mathbf{y}=\mathbf{x} / \varepsilon$ onde $\varepsilon>0$ é um parâmetro de pequeno valor que representa a microescala na qual as propriedades são alteradas (escala da microestrutura compósita), x e y são as coordenadas associadas com as dimensões macro- e micro- do material compósito, respectivamente (ver Fig. C.1).

Expandindo o deslocamento do piezocompósito $\mathbf{u}$ e o potencial elétrico $\phi$ assintoticamente (TELEGA, 1990), obtemos que:

$$
\begin{aligned}
\mathbf{u}^{\varepsilon} & =\mathbf{u}_{0}(\mathbf{x})+\varepsilon \mathbf{u}_{1}(\mathbf{x}, \mathbf{y}) \\
\phi^{\varepsilon} & =\phi_{0}(\mathbf{x})+\varepsilon \phi_{1}(\mathbf{x}, \mathbf{y})
\end{aligned}
$$

onde somente os termos variacionais de primeira ordem são considerados, uma vez que 


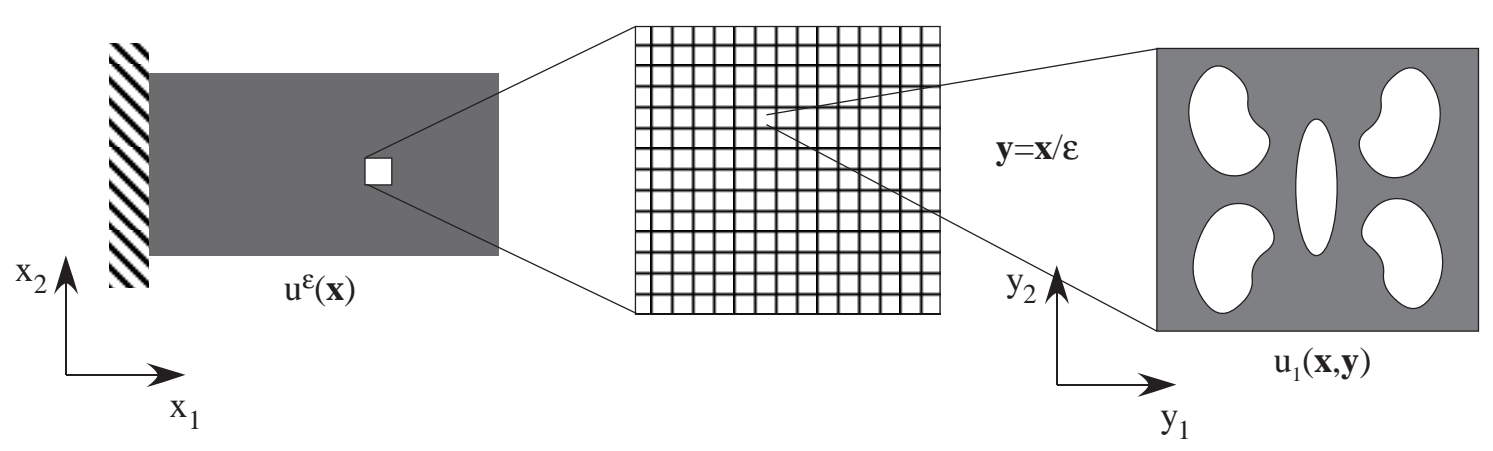

Componente (Macroescala) Microestrutura Periódica Célula Unitária (Microescala)

Figura 2.3: Conceito de Multi-escala ilustrando as dimensões macro (x) e micro (y).

supomos que no caso de aplicações dinâmicas, o comprimento de onda operacional é muito maior que as dimensões da célula unitária e $\mathbf{u}_{1}$ e $\phi_{1}$ são $Y$-periódicas. As tensões e os gradientes do potencial elétrico são escritas como:

$$
\begin{aligned}
\boldsymbol{\varepsilon}^{\varepsilon} & =\boldsymbol{\partial}_{\mathbf{x}} \mathbf{u}^{\varepsilon}=\boldsymbol{\partial}_{\mathbf{x}} \mathbf{u}_{0}(\mathbf{x})+\varepsilon \boldsymbol{\partial}_{\mathbf{x}} \mathbf{u}_{1}(\mathbf{x}, \mathbf{y})+\boldsymbol{\partial}_{\mathbf{y}} \mathbf{u}_{1}(\mathbf{x}, \mathbf{y}) \\
\boldsymbol{\nabla}_{\mathbf{x}} \phi^{\varepsilon} & =\boldsymbol{\nabla}_{\mathbf{x}} \phi_{0}(\mathbf{x})+\varepsilon \boldsymbol{\nabla}_{\mathbf{x}} \phi_{1}(\mathbf{x}, \mathbf{y})+\boldsymbol{\nabla}_{\mathbf{y}} \phi_{1}(\mathbf{x}, \mathbf{y})
\end{aligned}
$$

onde $\varepsilon$ é a tensão mecânica (ver Apêndice C) e:

$$
\left(\partial_{x}\right)_{i j}(.)=\frac{1}{2}\left(\frac{\partial(.)_{i}}{\partial x_{j}}+\frac{\partial(.)_{j}}{\partial x_{i}}\right) \quad\left(\partial_{y}\right)_{i j}(.)=\frac{1}{2}\left(\frac{\partial(.)_{i}}{\partial y_{j}}+\frac{\partial(.)_{j}}{\partial y_{i}}\right)
$$

A Eq. (2.2), junto com as propriedades da Eq. (2.1), deve ser substituída no funcional de energia para o meio elástico e no variacional deste funcional, em relação a $\mathbf{u}^{\varepsilon}$ (ver Apêndice C). Considerando o limite $\varepsilon \rightarrow 0$, temos que (SANCHEZ-PALENCIA, 1980),

$$
\lim _{\varepsilon \rightarrow 0} \int_{\Omega} \Phi\left(\mathbf{x}, \frac{\mathbf{x}}{\varepsilon}\right) d \Omega=\frac{1}{|Y|} \int_{\Omega} \int_{Y} \Phi\left(\mathbf{x}, \frac{\mathbf{x}}{\varepsilon}\right) d Y d \Omega
$$

onde $\Phi$ denota o funcional de interesse. Uma vez que os termos $\delta \mathbf{u}_{0}(\mathbf{x}), \delta \mathbf{u}_{1}(\mathbf{x}, \mathbf{y}), \delta \phi_{0}(\mathbf{x})$ e $\delta \phi_{1}(\mathbf{x}, \mathbf{y})$ são independentes, a Eq. (C.9) deve ser satisfeita pelas seguintes equações:

$$
\begin{gathered}
\int_{\Omega} \frac{1}{|Y|} \int_{Y} \partial_{\mathbf{x}} \delta \mathbf{u}_{0}(\mathbf{x}): \mathbf{c}^{E}(\mathbf{x}, \mathbf{y}):\left[\partial_{\mathbf{x}} \mathbf{u}_{0}(\mathbf{x})+\partial_{\mathbf{y}} \mathbf{u}_{1}(\mathbf{x}, \mathbf{y})\right] d Y d \Omega+ \\
+\int_{\Omega} \frac{1}{|Y|} \int_{Y} \partial_{\mathbf{x}} \delta \mathbf{u}_{0}(\mathbf{x}): \mathbf{e}(\mathbf{x}, \mathbf{y})\left[\nabla_{\mathbf{x}} \phi_{0}(\mathbf{x})+\nabla_{\mathbf{y}} \phi_{1}(\mathbf{x}, \mathbf{y})\right] d Y d \Omega- \\
-\int_{\Omega} \frac{1}{|Y|} \int_{Y} \mathbf{b}(\mathbf{x}, \mathbf{y}) \delta \mathbf{u}_{0}(\mathbf{x}) d Y d \Omega-\int_{\Gamma} \mathbf{t} \delta \mathbf{u}_{0}(\mathbf{x}) d \Gamma+ \\
+\int_{\Omega} \frac{1}{|Y|} \int_{Y} \rho(\mathbf{x}, \mathbf{y}) \ddot{\mathbf{u}}_{0}(\mathbf{x}) \delta \mathbf{u}_{0}(\mathbf{x}) d Y d \Omega=0 \\
\int_{\Omega} \frac{1}{|Y|} \int_{Y}\left[\partial_{\mathbf{x}} \mathbf{u}_{0}(\mathbf{x})+\partial_{\mathbf{y}} \mathbf{u}_{1}(\mathbf{x}, \mathbf{y})\right]: \mathbf{e}(\mathbf{x}, \mathbf{y}) \nabla_{\mathbf{x}} \delta \phi_{0}(\mathbf{x}) d Y d \Omega-
\end{gathered}
$$




$$
\begin{aligned}
&-\int_{\Omega} \frac{1}{|Y|} \int_{Y}\left[\nabla_{\mathbf{x}} \phi_{0}(\mathbf{x})+\nabla_{\mathbf{y}} \phi_{1}(\mathbf{x}, \mathbf{y})\right] \varepsilon^{S}(\mathbf{x}, \mathbf{y}) \nabla_{\mathbf{x}} \delta \phi_{0}(\mathbf{x}) d Y d \Omega+ \\
&+\int_{\Gamma} d \delta \phi(\mathbf{x}) d \Gamma=0
\end{aligned}
$$

para qualquer valor admissível de $\delta \mathbf{u}_{0}(\mathbf{x})$ e $\delta \phi_{0}(\mathbf{x})$, e:

$$
\begin{aligned}
& \int_{\Omega} \frac{1}{|Y|} \int_{Y} \partial_{\mathbf{y}} \delta \mathbf{u}_{1}(\mathbf{x}, \mathbf{y}): \mathbf{c}^{E}(\mathbf{x}, \mathbf{y}):\left[\partial_{\mathbf{x}} \mathbf{u}_{0}(\mathbf{x})+\partial_{\mathbf{y}} \mathbf{u}_{1}(\mathbf{x}, \mathbf{y})\right] d Y d \Omega+ \\
& +\int_{\Omega} \frac{1}{|Y|} \int_{Y} \partial_{\mathbf{y}} \delta \mathbf{u}_{1}(\mathbf{x}, \mathbf{y}): \mathbf{e}(\mathbf{x}, \mathbf{y})\left[\nabla_{\mathbf{x}} \phi_{0}(\mathbf{x})+\nabla_{\mathbf{y}} \phi_{1}(\mathbf{x}, \mathbf{y})\right] d Y d \Omega=0 \\
& \int_{\Omega} \frac{1}{|Y|} \int_{Y}\left[\partial_{\mathbf{x}} \mathbf{u}_{0}(\mathbf{x})+\partial_{\mathbf{y}} \mathbf{u}_{1}(\mathbf{x}, \mathbf{y})\right]: \mathbf{e}(\mathbf{x}, \mathbf{y}) \nabla_{\mathbf{y}} \delta \phi_{1}(\mathbf{x}, \mathbf{y}) d Y d \Omega- \\
& -\int_{\Omega} \frac{1}{|Y|} \int_{Y}\left[\nabla_{\mathbf{x}} \phi_{0}(\mathbf{x})+\nabla_{\mathbf{y}} \phi_{1}(\mathbf{x}, \mathbf{y})\right] \varepsilon^{S}(\mathbf{x}, \mathbf{y}) \nabla_{\mathbf{y}} \delta \phi_{1}(\mathbf{x}, \mathbf{y}) d Y d \Omega=0
\end{aligned}
$$

para qualquer valor admissível de $\delta \mathbf{u}_{1}(\mathbf{x}, \mathbf{y})$ e $\delta \phi_{1}(\mathbf{x}, \mathbf{y})$.

As Eq. (2.6) e (2.7) são denominadas por equações macroscópicas, uma vez que contém termos relacionados a $\delta \mathbf{u}_{0}(\mathbf{x})$ e $\delta \phi_{0}(\mathbf{x})$, enquanto que as Eq. (2.8) e (2.9) são denominadas por equações microscópicas, uma vez que contém termos relacionados a $\delta \mathbf{u}_{1}(\mathbf{x}, \mathbf{y})$ e $\delta \phi_{1}(\mathbf{x}, \mathbf{y})$. Devido à linearidade do problema e assumindo a separação das variáveis por $\mathbf{u}_{1}(\mathbf{x}, \mathbf{y})$ e $\phi_{1}(\mathbf{x}, \mathbf{y})$ (TELEGA, 1990), obtém-se que:

$$
\begin{aligned}
\mathbf{u}_{1} & =\chi(\mathbf{x}, \mathbf{y}) \varepsilon\left(\mathbf{u}_{0}(\mathbf{x})\right)+\Phi(\mathbf{x}, \mathbf{y}) \boldsymbol{\nabla}_{\mathbf{x}} \phi_{0}(\mathbf{x}) \\
\phi_{1} & =\psi(\mathbf{x}, \mathbf{y}) \varepsilon\left(\mathbf{u}_{0}(\mathbf{x})\right)+R(\mathbf{x}, \mathbf{y}) \boldsymbol{\nabla}_{\mathbf{x}} \phi_{0}(\mathbf{x}) \\
\boldsymbol{\partial}_{\mathbf{y}} \mathbf{u}_{1}(\mathbf{x}, \mathbf{y}) & =\boldsymbol{\partial}_{\mathbf{y}} \chi(\mathbf{x}, \mathbf{y}) \boldsymbol{\partial}_{\mathbf{x}} \mathbf{u}_{0}(\mathbf{x})+\boldsymbol{\partial}_{\mathbf{y}} \boldsymbol{\Phi}(\mathbf{x}, \mathbf{y}) \boldsymbol{\nabla}_{\mathbf{x}} \phi_{0}(\mathbf{x}) \\
\boldsymbol{\nabla}_{\mathbf{y}} \phi_{1}(\mathbf{x}, \mathbf{y}) & =\boldsymbol{\nabla}_{\mathbf{y}} \psi(\mathbf{x}, \mathbf{y}) \boldsymbol{\partial}_{\mathbf{x}} \mathbf{u}_{0}(\mathbf{x})+\boldsymbol{\nabla}_{\mathbf{y}} R(\mathbf{x}, \mathbf{y}) \boldsymbol{\nabla}_{\mathbf{x}} \phi_{0}(\mathbf{x})
\end{aligned}
$$

onde $\boldsymbol{\chi}(\mathbf{x}, \mathbf{y})$ é o deslocamento característico da célula unitária, $R(\mathbf{x}, \mathbf{y})$ é o potencial elétrico da célula unitária e $\psi(\mathbf{x}, \mathbf{y})$ e $\mathbf{\Phi}(\mathbf{x}, \mathbf{y})$ são as funções "acopladas" características da célula unitária. Todas estas funções são $Y$-periódicas; $\boldsymbol{\chi}(\mathbf{x}, \mathbf{y})$ e $\boldsymbol{\Phi}(\mathbf{x}, \mathbf{y})$ pertencem a $H_{p e r}\left(Y, R^{3}\right)$, e $\psi(\mathbf{x}, \mathbf{y})$ e $R(\mathbf{x}, \mathbf{y})$ pertencem a $H_{p e r}(Y)$, onde:

$$
\begin{aligned}
H_{p e r}(Y) & =\left\{v \in H^{1}(Y) \mid v \text { obtém valores iguais em lados opostos de } Y\right\}, \\
H_{\text {per }}\left(Y, R^{3}\right) & =\left\{\mathbf{v}=\left(v_{i}\right) \mid v_{i} \in H_{\text {per }}(Y), i=1,2,3\right\}
\end{aligned}
$$

que corresponde à condição de periodicidade da célula unitária.

A definição das propriedades efetivas é dada por (TELEGA, 1990): 


$$
\begin{aligned}
\mathbf{c}_{H}^{E} & =\frac{1}{|Y|}\left\{\int_{Y}\left[\mathbf{c}^{E}(\mathbf{x}, \mathbf{y}):\left(\mathbf{I}+\boldsymbol{\partial}_{\mathbf{y}} \boldsymbol{\chi}(\mathbf{x}, \mathbf{y})\right)+\mathbf{e}(\mathbf{x}, \mathbf{y}) \boldsymbol{\nabla}_{\mathbf{y}} \psi(\mathbf{x}, \mathbf{y})\right] d Y\right\} \\
\mathbf{e}_{H} & =\frac{1}{|Y|}\left\{\int_{Y}\left[\left(\mathbf{I}+\boldsymbol{\partial}_{\mathbf{y}} \boldsymbol{\chi}(\mathbf{x}, \mathbf{y})\right): \mathbf{e}(\mathbf{x}, \mathbf{y})-\boldsymbol{\varepsilon}^{s}(\mathbf{x}, \mathbf{y}) \boldsymbol{\nabla}_{\mathbf{y}} \psi(\mathbf{x}, \mathbf{y})\right] d Y\right\}= \\
& =\frac{1}{|Y|}\left\{\int_{Y}\left[\mathbf{c}^{E}(\mathbf{x}, \mathbf{y}): \boldsymbol{\partial}_{\mathbf{y}} \mathbf{\Phi}(\mathbf{x}, \mathbf{y})+\mathbf{e}(\mathbf{x}, \mathbf{y})\left(\mathbf{I}+\boldsymbol{\nabla}_{\mathbf{y}} R(\mathbf{x}, \mathbf{y})\right)\right] d Y\right\} \\
\boldsymbol{\varepsilon}_{H}^{S} & =\frac{1}{|Y|}\left\{\int_{Y}\left[\boldsymbol{\varepsilon}^{s}(\mathbf{x}, \mathbf{y})\left(\mathbf{I}+\boldsymbol{\nabla}_{\mathbf{y}} R(\mathbf{x}, \mathbf{y})\right)-\boldsymbol{\partial}_{\mathbf{y}} \mathbf{\Phi}(\mathbf{x}, \mathbf{y}): \mathbf{e}(\mathbf{x}, \mathbf{y})\right] d Y\right\}
\end{aligned}
$$

O cálculo das propriedades efetivas mostradas nas Eqs. (2.12), (2.13) e (2.14) podem ser vistas em (SILVA et al., 1999; SILVA; FONSECA; KIKUCHI, 1997; SILVA et al., 1999) para materiais piezocompósitos não-MGF. Os coeficientes piezelétricos homogeneizados dependem do tipo de classe cristalina considerada para o material piezelétrico básico. No caso desse estudo será considerada a classe hexagonal $6 \mathrm{~mm}$. Para simular a gradação contínua no interior dos elementos, as Eqs. (C.18) e (C.19) devem ser resolvidas utilizando o conceito de elementos finitos gradados mostrado nas Eqs. (2.18).

\subsubsection{Cálculo das tensões mecânicas na microestrutura}

Quando se utilizam materiais não-compósito, normalmente o projeto de estruturas considera apenas a máxima tensão principal nos critérios de projeto. Entretanto, quando são utilizados materiais compósitos, existem microconcentrações de tensões mecânicas no interior da célula unitária, principalmente em regiões de interface entre os materiais constituintes. O cálculo das tensões mecânicas na microestrutura de materiais compósitos muitas vezes é negligenciado e assume-se erroneamente apenas o valor da tensão mecânica "média". Este procedimento pode resultar em estruturas com maior propensão à fadiga ou à propagação de trincas.

O cálculo das microtensões mecânicas em materiais compósitos elásticos utilizando o Método da Homogeneização foi apresentado por Guedes e Kikuchi (1990). Neste trabalho é apresentado o cálculo considerando materiais piezelétricos. Assim, a equação da tensão mecânica para materiais piezelétricos pode ser reescrita em termos das propriedades homogeneizadas da seguinte forma:

$$
\mathbf{T}=\mathbf{c}_{H}^{E} \mathbf{S}-\mathbf{e}_{H} \mathbf{E}
$$

A deformação $\mathbf{S}$ e o campo elétrico $\mathbf{E}$ são expandidos assintoticamente conforme a Eq. (A.1) do Apêndice A. Das Eqs. (2.12), (2.13) e (2.14), pode-se ver que as propriedades efetivas elástica $\mathbf{c}_{H}^{E}$ e piezelétrica $\mathbf{e}_{H}$ são definidas pela homogeneização das propriedades microscópicas $\mathbf{c}^{E}(\mathbf{x}, \mathbf{y})$ e $\mathbf{e}(\mathbf{x}, \mathbf{y})$, respectivamente, no interior da célula 
unitária $Y$. Portanto, é possível calcular a distribuição das tensões mecânicas no interior da célula unitária através da Eq. (2.15):

$$
\begin{aligned}
\boldsymbol{\sigma}(\mathbf{x}, \mathbf{y}) & =\left[\mathbf{c}^{E}(\mathbf{x}, \mathbf{y}):\left(\mathbf{I}+\boldsymbol{\partial}_{\mathbf{y}} \chi(\mathbf{x}, \mathbf{y})\right)+\mathbf{e}(\mathbf{x}, \mathbf{y}) \boldsymbol{\nabla}_{\mathbf{y}} \psi(\mathbf{x}, \mathbf{y})\right] \partial_{\mathbf{x}} \mathbf{u}_{0}(\mathbf{x})- \\
& -\left[\mathbf{c}^{E}(\mathbf{x}, \mathbf{y}): \boldsymbol{\partial}_{\mathbf{y}} \mathbf{\Phi}(\mathbf{x}, \mathbf{y})+\mathbf{e}(\mathbf{x}, \mathbf{y})\left(\mathbf{I}+\boldsymbol{\nabla}_{\mathbf{y}} R(\mathbf{x}, \mathbf{y})\right)\right] \boldsymbol{\nabla}_{\mathbf{x}} \phi_{0}(\mathbf{x})
\end{aligned}
$$

\subsection{Formulação do Problema de Otimização Topológica}

As fontes de energia utilizadas atualmente, além de serem esgotáveis, são altamente poluentes, afetando o meio ambiente. Assim, novas fontes de energia, renováveis e limpas, tem sido amplamente pesquisadas. Uma delas baseia-se no uso de materiais piezelétricos. Uma aplicação interessante para os materiais piezelétricos são dispositivos de coleta de energia, que é um processo no qual a energia que seria desperdiçada no meio ambiente é transformada e armazenada para uso futuro, por outro dispositivo. A energia convertida pode ser empregada para estender a vida operacional de dispositivos tradicionalmente alimentados por baterias. Esta tecnologia é particularmente vantajosa em sistemas com acessibilidade limitada, tal como implantes biomédicos ou estruturas com microsensores sem fio, uma vez que tais dispositivos sejam capazes de gerar sua própria energia a partir do seu próprio ambiente. Assim, pode-se prolongar a vida útil de uma bateria pré-instalada, ou até mesmo substituí-la por completo.

O emprego de materiais piezelétricos em aplicações de coleta de energia a partir das vibrações do ambiente tem recebido significativa atenção na última década, com o objetivo de eliminar ou reduzir a necessidade de fontes de energia externa ou baterias em aparelhos eletrônicos em geral. O interesse na coleta de energia vibracional tem sido motivada pelos avanços tecnológicos de componentes eletrônicos de baixa potência, tais como sensores sem fio utilizados em monitoramento de saúde, sensores de pressão de pneus, aparelhos eletrônicos portáteis, ou veículos aéreos não tripulados (ERTURK; RENNO; INMAN, 2009). Outra motivação é o desejo de desenvolver dispositivos com dupla funcionalidade, tanto estrutural quanto para geração de energia, gerando assim economia de materiais e, consequentemente, redução de peso e custo. O interesse na coleta de energia utilizando materiais piezelétricos pode ser visto pelo aumento no número de artigos de revisão bibliográfica que tem sido publicados nos últimos anos (ANTON; SODANO, 2007; BEEBY; TUDOR; WHITE, 2006; COOK-CHENNAULT; THAMBI; SASTRY, 2008; PRIYA, 2007); maiores detalhes sobre aplicações, técnicas experimentais e abordagens de modelagem e projeto podem ser encontrados nessas referências.

Um exemplo é a companhia ferroviária East Japan Railway Company (JR East), 
que conduziu um experimento no início de 2008 numa estação de trem em Tóquio, onde um piso adaptado num local de grande movimentação de pessoas foi instalado próximo às catracas de acesso (ver Fig. 2.4). A partir das vibrações geradas pelo caminhar dos usuários de trem, a energia mecânica foi convertida em elétrica e foi empregada em dispositivos de sinalização e operação de catracas de acesso (KAWAI, 2006).
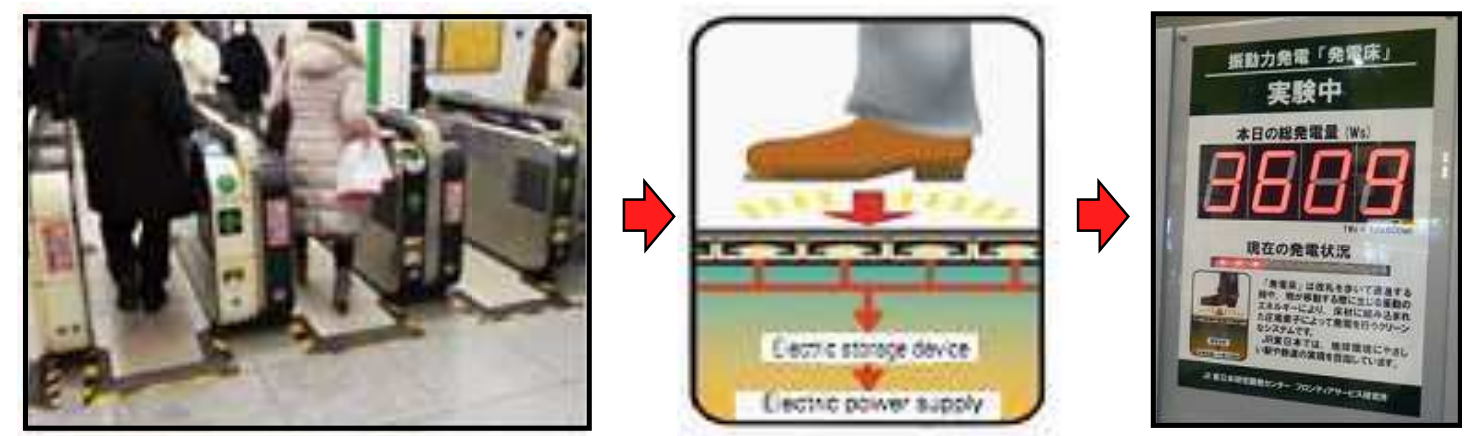

Figura 2.4: Exemplo de aplicação de coleta de energia (KAWAI, 2006).

As aplicações de coleta de energia podem ser modeladas, basicamente, através de um material piezelétrico sob um carregamento distribuído numa das faces, paralelo à direção de polarização (ver Fig. 2.5).

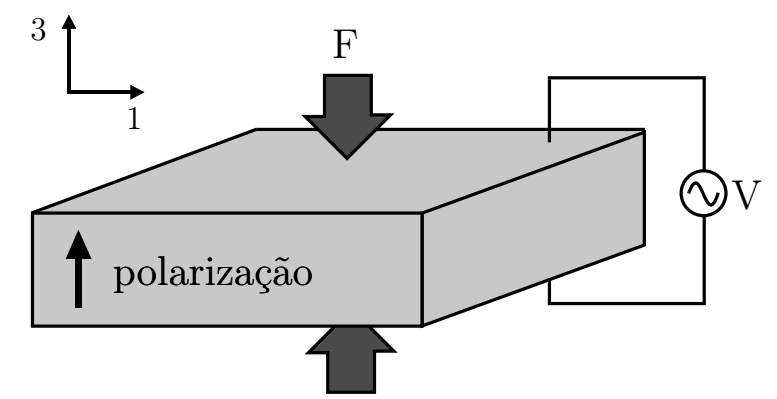

Figura 2.5: Modelo utilizado para definir os termos de $k$.

Portanto, o problema de otimização visando aplicações de coleta de energia em operação quasi-estática consiste em encontrar a distribuição de material que maximize o coeficiente de acoplamento eletromecânico $k$. Existem diversas formas de se calcular este coeficiente (ANSI/IEEE, 1984). No caso de projeto de materiais para aplicações quasi-estáticas, em um modelo bidimensional, assume-se que as deformações na célula unitária ocorram apenas na direção vertical. Assim, adota-se o coeficiente hidrostático k definido pela a Eq. (2.16) (SILVA et al., 1999):

$$
k=\sqrt{\frac{d_{33}^{2}}{s_{33}^{E}\left(\varepsilon_{33}^{S}+\frac{e_{33}^{2}}{c_{33}^{E}}\right)}}
$$

No caso do projeto de material, o domínio estendido de projeto é o domínio da célula unitária. O problema discreto, entretanto, onde a quantidade de material em cada elemento pode assumir apenas valores iguais a zero ou um é um problema mal-posto. 
Um modo típico de contornar isso em problemas de otimização topológica é relaxar o problema, permitindo que o material assuma valores de propriedade intermediários durante o processo de otimização. Para isso, deve-se definir um modelo de material adequado (CHERKAEV, 2000; ALLAIRE, 2002).

\subsubsection{Modelo de Material}

Os dois principais tipos de modelos de material que definem a mistura em microescala de dois ou mais materiais são (BENDSØE; SIGMUND, 1999): homogeneização e método de densidade. Neste projeto será utilizado o método das densidades. Entre os métodos de densidade, adota-se o modelo SIMP (Simple Isotropic Material with Penalization), o qual consiste em uma equação matemática que define o valor da propriedade do material em cada ponto do domínio em função de uma pseudo-densidade (que nos problemas de otimização topológica geralmente é a variável de projeto), e a propriedade básica do material a ser distribuído. Este modelo de material pode ser expresso por:

$$
\begin{aligned}
\mathbf{c}^{h} & =\rho^{p_{c}} \mathbf{c}_{\text {mat }_{A}}+\left(1-\rho^{p_{c}}\right) \mathbf{c}_{\text {mat }_{B}} \\
\mathbf{e}^{h} & =\rho^{p_{e}} \mathbf{e}_{\text {mat }_{A}}+\left(1-\rho^{p_{e}}\right) \mathbf{e}_{\text {mat }_{B}} \\
\varepsilon^{h} & =\rho^{p_{\varepsilon}} \boldsymbol{\varepsilon}_{\text {mat }_{A}}+\left(1-\rho^{p_{\varepsilon}}\right) \boldsymbol{\varepsilon}_{\text {mat }_{B}}
\end{aligned}
$$

onde $\mathbf{c}_{\text {mat }_{i}}, \mathbf{e}_{\text {mat }_{i}}$ e $\boldsymbol{\varepsilon}_{\text {mat }}$ correspondem aos tensores elástico, piezelétrico e dielétrico, respectivamente, dos materiais A e B. A variável $\rho$ é uma pseudo-densidade que descreve a quantidade dos materiais-base em cada ponto do domínio, a qual pode assumir valores entre 0 e 1. Uma solução topológica pode ser obtida aplicando coeficientes de penalização $p_{c}, p_{e}$ e $p_{\varepsilon}$ no modelo de material para recuperar a distribuição discreta 0-1, juntamente com técnicas de controle de gradiente na distribuição de material, tais como filtros ou técnicas de projeção (BENDSØE; SIGMUND, 2003). Segundo Kim et al. (2010), para que a convergência do problema seja estável, esses coeficientes de penalização devem ser escolhidos de forma que satisfaçam duas condições. A primeira é uma condição intrínseca que garante que, quanto mais material piezelétrico for utilizado, melhor será a eficiência na conversão de energia mecânica em elétrica. A segunda é uma condição dependente da função objetivo, que favorece uma distribuição distinta de material ao invés de uma distribuição de materiais intermediários no domínio de projeto, para uma mesma quantidade de material piezelétrico utilizada.

Alguns trabalhos publicados na literatura científica abordam também como variável de projeto o sentido da polarização de materiais piezelétricos (KOGL; SILVA, 2005; RUPP et al., 2009). A abordagem clássica vista na literatura utilizada para otimizar as direções 
de polarização em materiais piezelétricos é a definição de diversas polarizações locais como variáveis de projeto. Numa estrutura de elementos finitos, esta abordagem pode ser ilustrada conforme a Fig. 2.6, onde cada seta representa a direção de polarização local em cada elemento e é definida pelo ângulo $\theta_{i}$ relativo a um referencial fixo. Assim, as variáveis de projeto podem assumir valores contínuos dentro de um certo intervalo pré-definido. Entretanto, uma desvantagem desta abordagem é que espaço de solução global torna-se altamente não-convexo (STEGMANN; LUND, 2005).

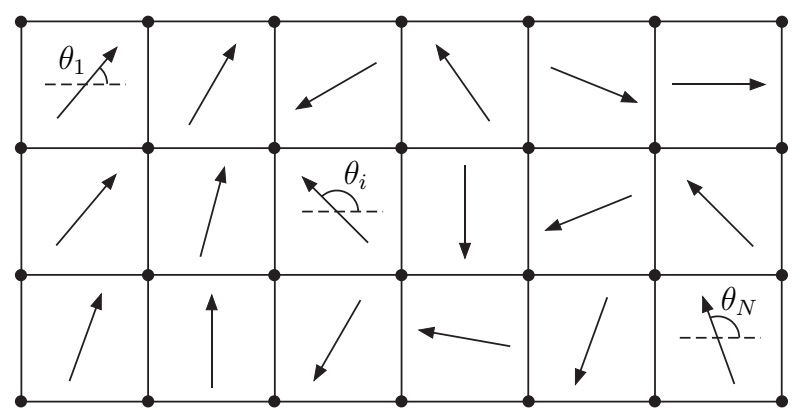

Figura 2.6: Esquemático do conceito clássico de otimização de orientação de polarização numa malha de elementos finitos. As setas indicam as direções de polarização em cada elemento, definidas pelas variáveis de projeto $\left(\theta_{i}\right)$.

O método denominado por Otimização de Material Discreto (OMD), apresentado por Stegmann e Lund (2005), é baseado na otimização topológica multifásica, utiliza gradientes combinados com programação matemática para resolver um problema discreto de otimização e reduz o risco de se obter soluções ótimas locais. Esta abordagem foi primeiramente introduzida como uma otimização topológica multifásica por Sigmund e Torquato (SIGMUND; TORQUATO, 1997), utilizada para projetar materiais com expansão térmica extrema e, posteriormente, para projetar materiais com módulo de Bulk (GIBIANSKY; SIGMUND, 2000). Neste trabalho, o OMD é utilizado para resolver problemas de orientação de polarização de materiais piezelétricos convertido num problema de seleção de materiais. A implementação deste método é descrita na Seção 2.3.4.

Dessa forma, o problema de otimização de piezocompósitos com gradação funcional visando aplicações de coleta de energia pode ser escrito como:

$$
\begin{array}{ll}
\underset{\rho(\mathbf{x}), \theta(\mathbf{x})}{\operatorname{Maximizar}:} & F(\rho(\mathbf{x}), \theta(\mathbf{x}))=k \\
& 0 \leq \rho(\mathbf{x}) \leq 1 \\
& -90^{\circ} \leq \theta(\mathbf{x}) \leq 90^{\circ} \\
& \text { condições de simetria } \\
& \text { controle de gradação e complexidade }
\end{array}
$$

onde $\rho(\mathbf{x})$ e $\theta(\mathbf{x})$ são as variáveis de projeto e $F$ é a função objetivo a ser maximizada. Não há a necessidade de um controle da rigidez, pois trata-se de uma otimização de dois 
materiais fisicamente existentes, ao invés de um material e vazio. As condições de simetria e de contorno são descritas na Seção 2.3.3.

\subsection{Implementação Numérica}

Nesta seção é apresentada a implementação numérica utilizada neste capítulo. Primeiramente, é apresentada a implementação numérica do método de homogeneização, conforme mostrado na Seção 2.1, onde as Eqs. (2.12), (2.13) e (2.14) das propriedades homogeneizadas são calculadas através do MEF. Em seguida é apresentado o cálculo das sensibilidades das propriedades homogeneizadas, as quais são utilizadas no método de otimização. Então o problema de otimização é formulado em termos do MEF e é apresentado o fluxograma de solução do problema. São apresentados a implementação do elemento finito poligonal, o método de Otimização de Material Discreto (OMD), o cálculo do coeficiente de acoplamento eletromecânico $(k)$ para coleta de energia, as condições de simetria e a formulação do problema de otimização para o projeto de materiais piezocompósitos.

\subsubsection{Elementos Finitos Gradados}

Nos materiais piezelétricos MGF as propriedades variam de forma contínua no interior do domínio. Essa variação contínua deve ser levada em conta na solução das equações de MEF. Na literatura, em geral (ALMAJID; TAYA; HUDNUT, 2001), essa variação contínua é aproximada por uma variação em camadas o que leva a discrepâncias na distribuição de tensões que se apresenta de forma descontínua no domínio. Esse problema pode ser contornado inicialmente implementando-se o conceito de elementos finitos gradados piezelétricos proposto por Silva et al. (SILVA; CARBONARI; PAULINO, 2007) tal que nas integrais de MEF as propriedades são integradas em conjunto avaliando-as nos pontos de Gauss. Esse conceito permite obter uma distribuição de tensões contínua coerente com a distribuição contínua das propriedades no MGF. O exemplo da fig. 2.7 mostra os efeitos de uma variação exponencial de propriedades quando são utilizados elementos em múltiplas camadas ou elementos finitos gradados.

Segundo Silva et al. (SILVA; CARBONARI; PAULINO, 2007) as propriedades do material (avaliadas em cada ponto de integração de Gauss) podem ser interpoladas a partir das propriedades do material nodal do elemento finito e utilizando as mesmas funções de forma que são utilizadas para os deslocamentos ou funções características (ver Eq. (2.27)).

Generalizando o conceito de elementos finitos gradados a materiais anisotrópicos e 


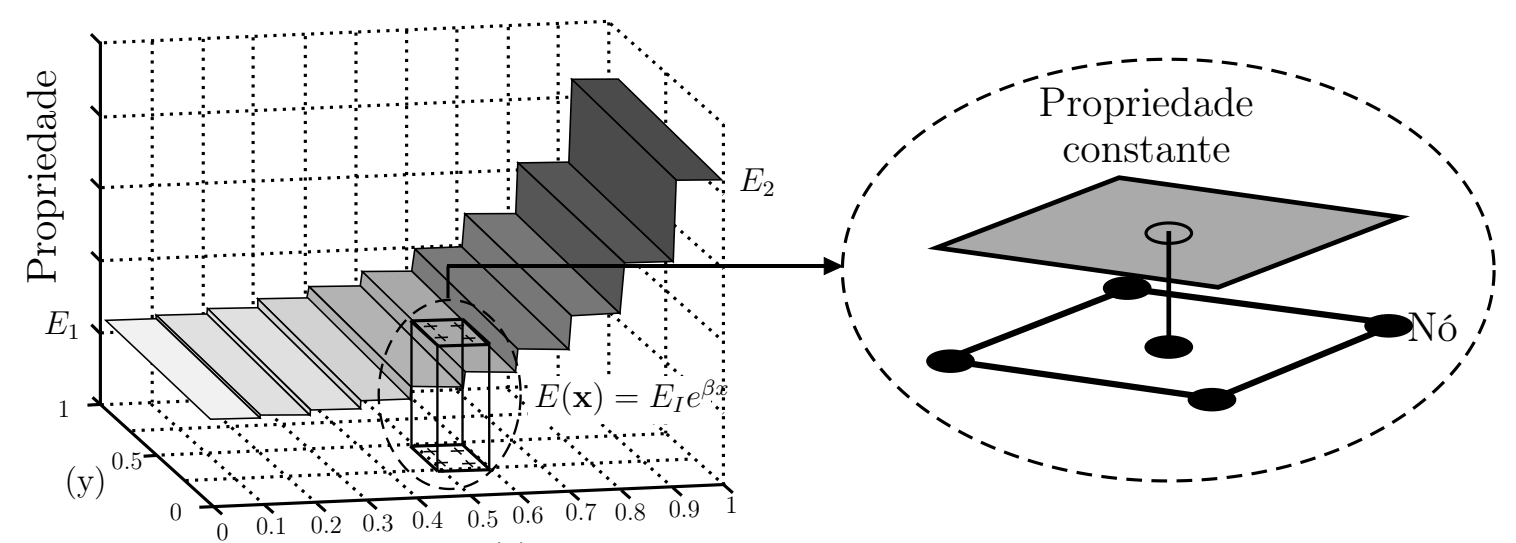

(x)

(a)

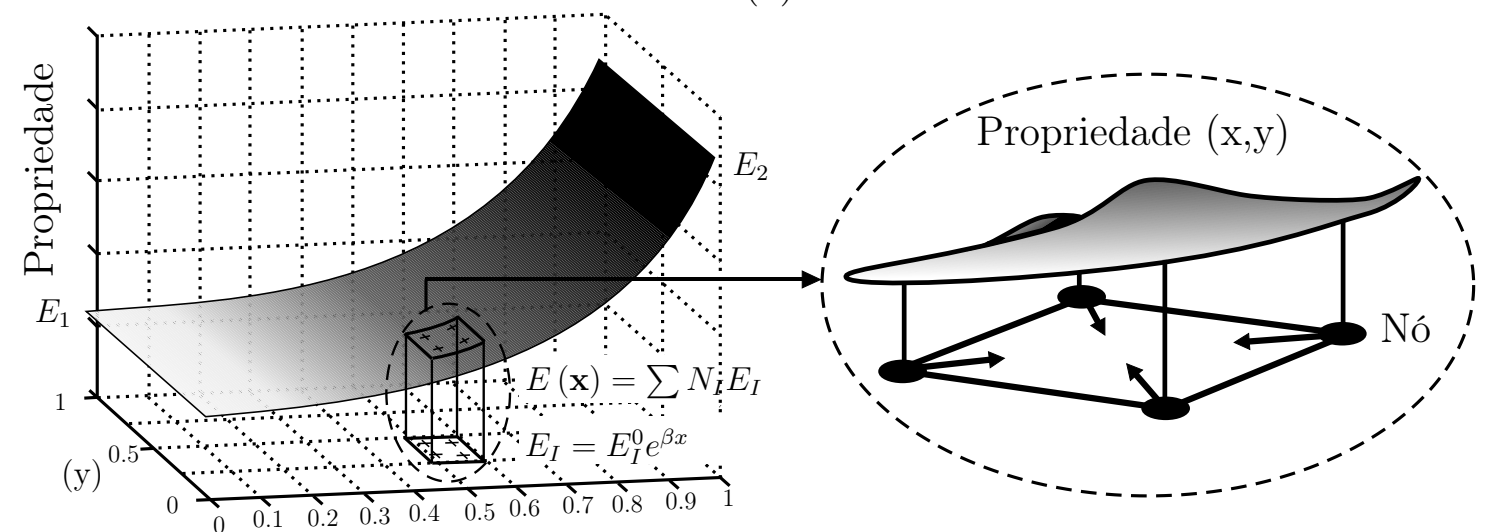

(x)

(b)

Figura 2.7: Conceito de elementos finitos gradados utilizando como exemplo uma variação exponencial de material. (a) Elemento Finito Homogêneo; (b) Elemento Finito Gradado (SILVA; CARBONARI; PAULINO, 2007).

considerando que a matriz de rigidez $\mathbf{K}_{u \boldsymbol{u}}$ de dimensões $6 \times 6$ envolve 9 constantes elásticas $\left(C_{i j}\right)$, a matriz piezelétrica $\mathbf{K}_{\boldsymbol{u} \phi}$ de dimensões $6 \times 3$ envolve 5 constantes piezelétricas $\left(e_{i j}\right)$ e a matriz dielétrica $\mathbf{K}_{\phi \phi}$ de dimensões $3 \times 3$ envolve 3 constantes dielétricas $\left(\varepsilon_{i j}\right)$, temos:

$$
\begin{array}{rlrl}
C_{i j} & =\sum_{k=1}^{m} N_{k}\left(C_{i j}\right)_{k} & \mathrm{p} / i=j=1 . .3 & \\
C_{44}=\sum_{k=1}^{m} N_{k}\left(C_{44}\right)_{k} & C_{55}=\sum_{k=1}^{m} N_{k}\left(C_{55}\right)_{k} & C_{66}=\sum_{k=1}^{m} N_{k}\left(C_{66}\right)_{k} \\
e_{15}=\sum_{k=1}^{m} N_{k}\left(e_{15}\right)_{k} & e_{24}=\sum_{k=1}^{m} N_{k}\left(e_{24}\right)_{k} & e_{i j}=\sum_{k=1}^{m} N_{k}\left(e_{i j}\right)_{k} \quad \mathrm{p} / i=3, j=1 . .3 \\
\varepsilon_{11}=\sum_{k=1}^{m} N_{k}\left(\varepsilon_{11}\right)_{k} & \varepsilon_{22}=\sum_{k=1}^{m} N_{k}\left(\varepsilon_{22}\right)_{k} & \varepsilon_{33}=\sum_{k=1}^{m} N_{k}\left(\varepsilon_{33}\right)_{k}
\end{array}
$$

O parâmetro $m$ destas equações é o número de pontos nodais no elemento e e $N_{i}$ são as funções de forma. Nestas equações a notação $e$ (elemento) tem sido omitida para simplificar as expressões.

As variáveis de projeto são definidas para cada nó do elemento, ao invés de cada 
elemento finito. Essa formulação é conhecida como CAMD (Continuous Approximation of Material Distribution) (MATSUI; TERADA, 2004; RAHMATALLA; SWAN, 2004) e pelo fato de permitir representar uma distribuição contínua de material de uma forma mais natural, é fortemente compatível com o conceito de MGF. Desse modo, a pseudo-densidade $(\rho)$ no interior de cada elemento finito é dada por

$$
\rho(\mathbf{x})=\sum_{I=1}^{n_{d}} \rho_{I} N_{I},
$$

onde $\rho_{I}$ é a variável de projeto nodal, $N_{I}$ é a função de forma do elemento finito que deve ser adotada para evitar valores não-negativos das variáveis de projeto e $n_{d}$ é o número de nós em cada elemento (por exemplo, quatro nós no caso bidimensional).

\subsubsection{Elementos finitos poligonais}

A maioria dos trabalhos publicados no tema de otimização topológica utiliza o elemento finito quadrilátero de 4 nós. Entretanto, este tipo de elemento apresenta alguns problemas numéricos, tais como instabilidade de xadrez e conexões de um nó entre elementos, os quais tem sido objetos de ampla pesquisa (SIGMUND; PETERSSON, 1998). Outro problema menos comum é que malhas estruturais podem ocasionar dependência de malha em orientações de membros, gerando topologias não-ótimas. Uma possível solução para evitar estes problemas numéricos é a utilização de malhas de elementos finitos poligonais construídas a partir de diagramas de Voronoi, as quais possuem alto grau de isotropia geométrica e oferecem alta flexibilidade de discretização em domínios complexos (TALISCHI et al., 2009). A Fig. 2.8 ilustra um exemplo de uma malha de elementos finitos poligonais. Com o auxílio do algoritmo de Lloyd (LLOYD, 1982), é possível evitar distorções excessivas dos elementos e obter malhas com elementos de tamanho uniforme.

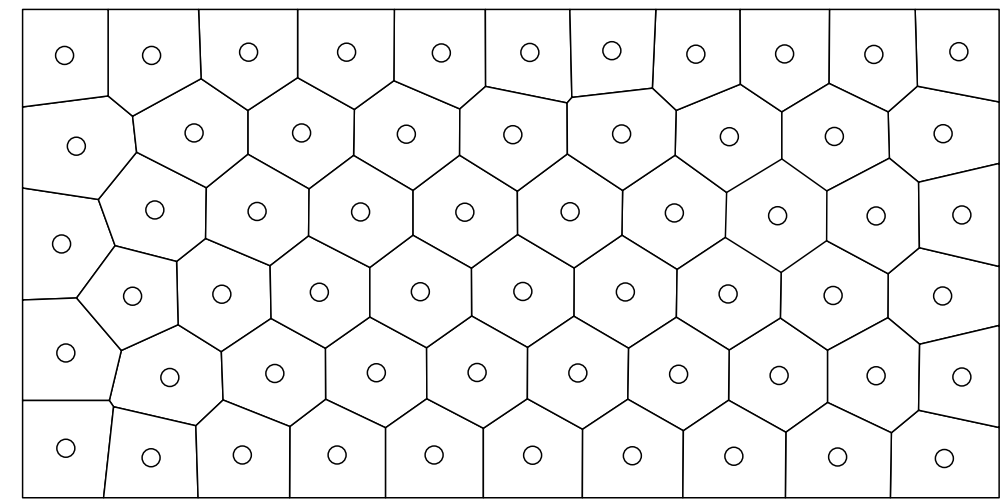

Figura 2.8: Exemplo de uma malha de elementos finitos poligonais, onde os pontos azuis são os pontos geradores.

O principal conceito de geração de malhas de elementos finitos poligonais é a escolha de um conjunto de pontos geradores, ou 'sementes', de tal forma que o diagrama de Voronoi 
incorpore as fronteiras do domínio. Neste trabalho, adota-se a formulação descrita por Talischi et al (TALISCHI et al., 2009), estendida para análise de materiais piezelétricos.

\section{- Conceitos e definições}

Dado um conjunto de pontos geradores $P=\left\{\mathbf{p}_{i}\right\}_{i=1}^{n}$ existente num domínio $\Omega \subseteq \mathbb{R}^{d}$ (ver Fig. 2.8, o diagrama de Voronoi $P$, definido por $\mathcal{V}(P)$, é a divisão do domínio $\Omega$ em $n$ regiões definidas por

$$
V_{i}=\bigcap_{\forall j, j \neq i}\left\{\mathbf{x} \in \Omega \mid \delta\left(\mathbf{x}, \mathbf{p}_{i}\right) \leq \delta\left(\mathbf{x}, \mathbf{p}_{j}\right)\right\}, i=1, \ldots, n
$$

onde $\delta(\cdot, \cdot)$ é a distância euclidiana. Em outras palavras, a célula de Voronoi $V_{i}$ é composta pelos pontos que estão mais próximos a $\mathbf{p}_{i}$ do que qualquer outro ponto em $P$. Nota-se que cada célula $V_{i}$ é não-vazia, uma vez que $\mathbf{p}_{i} \in V_{i}$. Além disso, todas as células de Voronoi são necessariamente polígonos convexos, uma vez que são formadas pela intersecção de meio-planos. Isto é importante, pois a formulação de discretização utilizada neste trabalho é restrita à polígonos convexos. O diagrama de Voronoi é de ponto centróide se cada ponto gerador coincide com o centróide da célula de Voronoi correspondente, isto é,

$$
\mathbf{p}_{i}=\overline{\mathbf{p}}_{i} \quad \forall_{\mathrm{i}}=1, \ldots \mathrm{n} \quad \text { where } \quad \overline{\mathbf{p}}_{i}:=\frac{\int_{V_{i}} \mathbf{x} \mu(\mathbf{x}) d x}{\int_{V_{i}} \mu(\mathbf{x}) d x}
$$

e $\mu(\mathbf{x})$ é dado pela função densidade sobre o domínio $\Omega$ (DU; FABER; GUNZBURGER, 1999). Para $\mu(\mathbf{x}) \equiv 1, \mathbf{p}_{i}$ é o centro geométrico de $V_{i}$.

Um dos algoritmos mais populares para se construir um diagrama de Voronoi de ponto centróide é um método iterativo denominado por algoritmo de Lloyd (LLOYD, 1982), pois é convergente localmente (DU; EMELIANENKO; JU, 2006). A versão determinística do algoritmo de Lloyd pode ser dada da seguinte forma:

- Construir o diagrama de Voronoi $\mathcal{V}(P)$ associado a $P=\left\{\mathbf{p}_{i}\right\}_{i=1}^{n}$.

- Calcular o centróide da cada célula Voronoi $\overline{\mathbf{p}}_{i}$.

- Substituir o conjunto de pontos geradores inicial $P$ pelos centróides $\bar{P}=\left\{\overline{\mathbf{p}}_{i}\right\}_{i=1}^{n}$ e voltar ao passo 1 até que a convergência seja atingida.

O critério de convergência pode ser baseado na redução dos deslocamentos do pontos geradores. 


\section{- Algoritmo de Geração da Malha Poligonal}

A malha de elementos finitos poligonais pode ser gerada a partir de um conjunto de pontos do domínio $\Omega$, incluindo pontos adicionais de forma que o diagrama de Voronoi resultante forme uma aproximação na fronteira $\partial \Omega$. O procedimento proposto em (BOLANDER; SAITO, 1998; YIP; MOHLE; BOLANDER, 2005) é resumido a seguir:

- O interior de $\Omega$ é preenchido por um determinado número de pontos 'sementes'. Este conjunto de pontos adicionais é denominado por $P_{\text {int }}$.

- Para gerar uma aproximação nas fronteiras do domínio, os pontos do interior são refletidos em relação às arestas do domínio. Este conjunto de pontos auxiliares são denominados por $P_{a u x}$.

- Gera-se o diagrama de Voronoi do conjunto de pontos $P=P_{\text {int }} \cup P_{\text {aux }}$.

- Obtém-se uma discretização poligonal a partir das células associadas aos pontos $P_{\text {int }}$.

Nota-se que se um ponto 'semente' e o seu reflexo possuir células de Voronoi adjacentes, a aresta em comum entre elas se alinha com a fronteira $\partial \Omega$. Este procedimento é ilustrado na Fig. 2.9, numa região quadrilátera, para um conjunto de 10 pontos aleatórios.

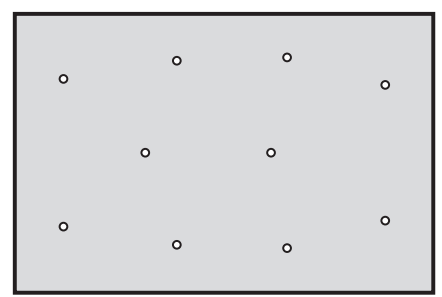

(a)

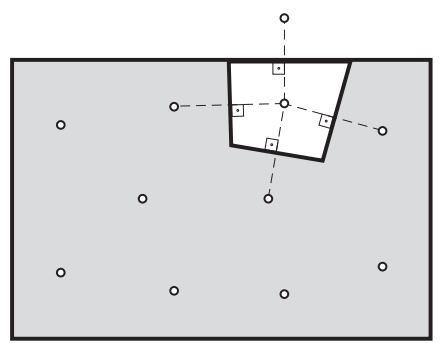

(b)

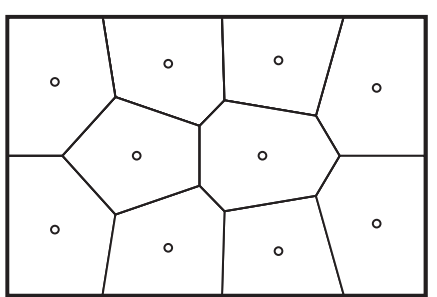

(c)

Figura 2.9: Procedimento para geração do diagrama de Voronoi: (a) o interior do domínio é preenchido com 10 pontos 'sementes' (círculos); (b) espelham-se os pontos

auxiliares e traça-se a célula de Voronoi, para um dado ponto 'semente' (pontos auxiliares desnecessários omitidos); (c) diagrama de Voronoi completo no interior do domínio.

\section{- Formulação do Elemento Poligonal}

Nesta seção, resume-se brevemente a formulação do elemento finito para polígonos convexos descrita em (SUKUMAR; TABARRAEI, 2004). Esta formulação gera um espaço de conformação de malhas poligonais utilizando funções de interpolação dos vizinhos naturais e transformações isoparamétricas. 
Considere um conjunto de nós $\left\{\mathbf{q}_{i}\right\}$ a de pontos $\mathbf{p}$, onde quer-se interpolar as informações nodais. Os pontos $\mathbf{p}$ e $\mathbf{q}_{i}$ são 'vizinhos naturais' se suas células de Voronoi tem uma aresta em comum (SUKUMAR; TABARRAEI, 2004). Define-se o conjunto de vizinhos naturais de $\mathbf{p}$ da seguinte forma:

$$
\mathcal{I}(\mathbf{p})=\left\{i \mid V_{i} \cap V_{p} \neq \emptyset\right\}
$$

onde $V_{i}$ e $V_{p}$ denotam as células de Voronoi de $\mathbf{q}_{i}$ e $\mathbf{p}$, respectivamente. O interpolador de Laplace correspondente a $\mathbf{q}_{i}$ é dado por:

$$
\phi_{i}(\mathbf{x})=\frac{w_{i}(\mathbf{x})}{\sum_{j \in \mathcal{I}} w_{j}(\mathbf{x})} \quad \text { onde } \quad \mathrm{w}_{\mathrm{i}}(\mathbf{x})=\frac{\mathrm{s}_{\mathrm{i}}(\mathbf{x})}{\mathrm{h}_{\mathrm{i}}(\mathbf{x})}
$$

Aqui, $\mathbf{x}$ são as coordenadas de $\mathbf{p}, s_{i}$ é a distância entre $V_{i}$ e $V_{p}$ e $h_{i}$ denota a distância entre $\mathbf{p}$ e $\mathbf{q}_{i}$, conforme ilustrado na Fig. 2.10. Pela construção, as funções de Laplace são não-negativas, contínuas e atendem o critério de completude:

$$
0 \leq \phi_{i}(\mathbf{x}) \leq 1, . \quad \sum_{i \in \mathcal{I}} \phi_{i}(\mathbf{x})=1
$$

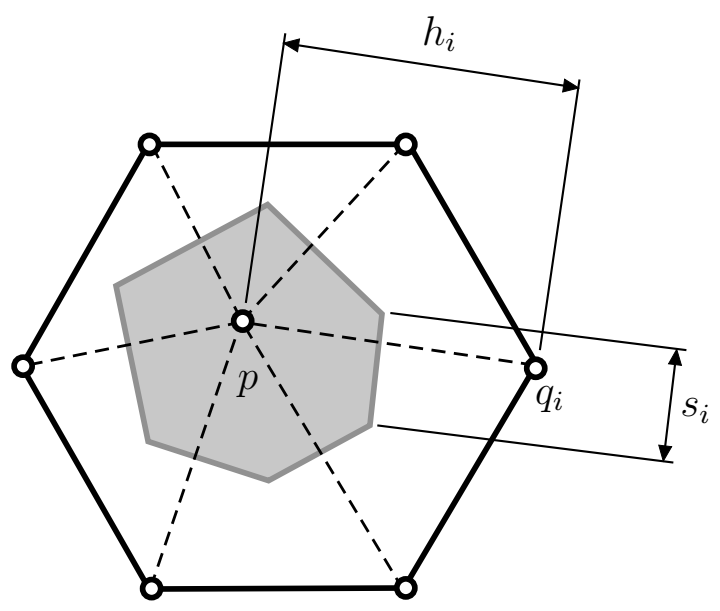

Figura 2.10: Num polígono convexo, todo ponto interior é um vizinho natural de todos os vértices. As dimensões geométricas $s_{i}$ e $h_{i}$ utilizadas para definir as funções de forma de Laplace estão mostradas aqui.

Além disso, pode ser demonstrado que estas funções são linearmente precisas:

$$
\sum_{i \in \mathcal{I}} \mathbf{x}_{i} \phi_{i}(\mathbf{x})=\mathbf{x}
$$

Nesta equação, $\mathbf{x}_{i}$ representa as coordenadas do nó $\mathbf{q}_{i}$. Além disso, as funções de Laplace são lineares nas fronteiras do elemento e satisfazem a propriedade do delta de Kronecker: 


$$
\phi_{i}\left(\mathbf{x}_{j}\right)=\delta_{i j}
$$

Isto significa que o valor interpolado num nó é igual ao valor nodal. A Fig. 2.11 mostra uma típica função de forma de Laplace. Se os nós forem posicionados nos vértices do polígono convexo, qualquer ponto interior do polígono tem $\left\{\mathbf{q}_{i}\right\}$ como vizinhos naturais. Portanto, as funções de forma de Laplace correspondentes a $\left\{\mathbf{q}_{i}\right\}$ formam um elemento finito poligonal.

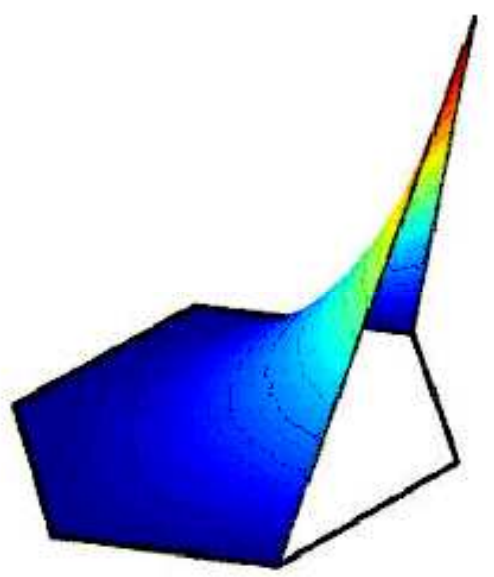

Figura 2.11: Típica função de forma de Laplace para um hexágono regular.

\subsubsection{Método da homogeneização}

As Eqs. (2.12), (2.13) e (2.14) das propriedades homogeneizadas são resolvidas utilizando o MEF. Assumimos que a célula unitária é discretizada por $N$ elementos finitos, ou seja:

$$
Y=\bigcup_{n=1}^{N} \Omega^{e}
$$

onde $\Omega^{e}$ é o domínio de cada elemento. Um elemento bilinear poligonal com três graus de liberdade por nó (dois deslocamentos e um potencial elétrico) é empregado na implementação do elemento finito bidimensional. É implementado também o conceito de elementos finitos gradados, descrito na Seção 2.3.1. São consideradas funções de interpolação bilineares para deslocamentos e potenciais elétricos. Portanto, as funções características previamente definidas são expressas para cada elemento em função das funções de forma $\left(N_{I}\right)$ :

$$
\begin{aligned}
\chi_{i}^{(m n)} \cong N_{I} \chi_{i I}^{(m n)} & \psi^{(m n)} \cong N_{I} \psi_{I}^{(m n)} & \\
\Phi_{i}^{(m)} \cong N_{I} \Phi_{i I}^{(m)} & R^{(m)} \cong N_{I} R_{I}^{(m)} & I=1, N N
\end{aligned}
$$


onde $N N$ é o número de nós por elemento finito (neste caso, quatro). Relações similares são escritas para os deslocamentos virtuais v e potencial elétrico virtual $\varphi$. Substituindo as relações (2.27) nas Eq. (C.20) a (C.23) e montando matrizes individuais para cada elemento, obtemos o seguinte sistema matricial global para cada carregamento $m n$ ou $m$ (SILVA et al., 1999; SILVA; FONSECA; KIKUCHI, 1997; SILVA et al., 1999):

$$
\begin{aligned}
{\left[\begin{array}{cc}
\mathbf{K}_{\mathbf{u u}} & \mathbf{K}_{\mathbf{u} \phi} \\
\mathbf{K}_{\mathbf{u} \phi}^{t} & -\mathbf{K}_{\phi \phi}
\end{array}\right]\left\{\begin{array}{l}
\widehat{\boldsymbol{\chi}}^{(m n)} \\
\widehat{\boldsymbol{\psi}}^{(m n)}
\end{array}\right\}=\left\{\begin{array}{l}
\mathbf{F}^{(m n)} \\
\mathbf{Q}^{(m n)}
\end{array}\right\} } \\
{\left[\begin{array}{cc}
\mathbf{K}_{\mathbf{u u}} & \mathbf{K}_{\mathbf{u} \phi} \\
\mathbf{K}_{\mathbf{u} \phi}^{t} & -\mathbf{K}_{\phi \phi}
\end{array}\right]\left\{\begin{array}{l}
\widehat{\boldsymbol{\Phi}}^{(m)} \\
\widehat{\mathbf{R}}^{(m)}
\end{array}\right\}=\left\{\begin{array}{l}
\mathbf{F}^{(m)} \\
\mathbf{Q}^{(m)}
\end{array}\right\} }
\end{aligned}
$$

onde $\widehat{\boldsymbol{\chi}}, \widehat{\boldsymbol{\psi}}, \widehat{\boldsymbol{\Phi}}$ e $\widehat{\mathbf{R}}$ são os valores nodais correspondentes das funções características $\boldsymbol{\chi}$, $\boldsymbol{\psi}, \boldsymbol{\Phi}$ e $\mathbf{R}$, respectivamente. As matrizes de rigidez, piezelétrica e dielétrica globais $\left(\mathbf{K}_{\mathbf{u u}}\right.$, $\mathbf{K}_{\mathbf{u} \phi}$ e $\mathbf{K}_{\phi \phi}$, respectivamente) são montadas a partir das matrizes individuais de cada elemento e os vetores de força e carga elétrica globais (F e $\mathbf{Q})$ são montados a partir dos vetores individuais de força de cada elemento:

$$
\begin{array}{rlrl}
\mathbf{K}_{\mathbf{u u}} & =A_{e=1}^{N} \mathbf{K}_{\mathbf{u u}}^{e} & \mathbf{K}_{\mathbf{u} \phi}=A_{e=1}^{N} \mathbf{K}_{\mathbf{u} \phi}^{e} & \mathbf{K}_{\phi \phi}=A_{e=1}^{N} \mathbf{K}_{\phi \phi}^{e} \\
\mathbf{F} & =A_{e=1}^{N} \mathbf{F}^{e} & \mathbf{Q}=A_{e=1}^{N} \mathbf{Q}^{e} &
\end{array}
$$

As matrizes e vetores do elemento são dados pelas seguintes expressões:

$$
\begin{array}{rlrl}
K_{u u(i I j J)}^{e} & =\int_{\Omega^{e}} c_{i p j q}^{E} \frac{\partial N_{I}}{\partial y_{p}} \frac{\partial N_{J}}{\partial y_{q}} d \Omega^{e} \quad ; \quad K_{u \phi(i I J)}^{e}=\int_{\Omega^{e}} e_{k i j} \frac{\partial N_{I}}{\partial y_{j}} \frac{\partial N_{J}}{\partial y_{k}} d \Omega^{e} \\
K_{\phi \phi(I J)}^{e} & =\int_{\Omega^{e}} \varepsilon_{i j}^{S} \frac{\partial N_{I}}{\partial y_{i}} \frac{\partial N_{J}}{\partial y_{j}} d \Omega^{e} & & \\
F_{i I}^{e(m n)} & =-\int_{\Omega^{e}} c_{i j m n}^{E} \frac{\partial N_{I}}{\partial y_{j}} d \Omega^{e} \quad ; \quad Q_{I}^{e(m n)}=-\int_{\Omega^{e}} e_{k m n} \frac{\partial N_{I}}{\partial y_{k}} d \Omega^{e} \\
F_{i I}^{e(m)} & =-\int_{\Omega^{e}} e_{m i j} \frac{\partial N_{I}}{\partial y_{j}} d \Omega^{e} \quad ; \quad Q_{I}^{e(m)}=\int_{\Omega^{e}} \varepsilon_{m j}^{S} \frac{\partial N_{I}}{\partial y_{j}} d \Omega^{e}
\end{array}
$$

Em problemas bidimensionais existem cinco casos de carregamento a serem resolvidos independentemente. Três deles surgem das Eq. (C.24) e (C.25), onde os índices $m$ e $n$ podem ser 1 ou 3, resultando nas combinações 11, 33 e 13 ou 31, para mn. Os outros dois casos de carregamento surgem das Eq. (C.26) e (C.27) onde o índice $m$ pode ser 1 e 3. Todos os casos de carregamento devem ser resolvidos sob a condição de contorno de periodicidade na célula unitária para os deslocamentos e potenciais elétricos (SILVA et al., 1999; SILVA; FONSECA; KIKUCHI, 1997; SILVA et al., 1999). Caso a célula unitária possua alguma simetria relativa aos eixos $x$ e $z$, é possível reduzir o custo computacional. Neste caso, o processo de otimização e homogeneização é realizado apenas em uma parte do 
domínio de projeto.

No caso do estado plano de deformação, se a célula unitária for simétrica em relação a um ou dois eixos os coeficientes homogeneizados serão ao menos ortotrópicos, uma vez que o material piezelétrico é ortotrópico e está presente na célula unitária. Se não houver nenhuma simetria os coeficientes homogeneizados serão anisotrópicos. Se o piezocompósito for anisotrópico, o número de coeficientes homogeneizados é reduzido para quatro coeficientes elásticos e dois coeficientes dielétricos para o caso 2D. Já os coeficientes homogeneizados piezelétricos dependem do tipo de classe cristalina do material piezelétrico de base. Para a classe hexagonal $6 \mathrm{~mm}$ considerada neste estudo, existem três coeficientes homogeneizados piezelétricos para o caso 2D. As condições de simetria são impostas nas funções características $\boldsymbol{\chi}, \boldsymbol{\psi} \Phi$ e $R$, apresentadas na Eq. (C.16). No estado plano de deformação existem cinco casos de carregamento a serem resolvidos no problema de homogeneização. Para cada um desses casos são apresentadas as condições de simetria para as funções características (deslocamentos e potenciais elétricos) nas Tabelas C.1 e C.2. Estas condições de contorno foram obtidas ampliando as condições de contorno de microestruturas elásticas (SILVA et al., 1999; SILVA; FONSECA; KIKUCHI, 1997; SILVA et al., 1999) para o caso piezelétrico a partir das Eq. (C.39), (C.40) e (C.41). Estas condições de contorno assumem que a célula unitária é ortotrópica com eixo de polarização piezelétrica na direção $z$ (ou 3). No caso do modelo discreto, as condições de contorno dos cinco casos de carregamento para o caso de simetria de $1 / 4$ de célula unitária estão ilustradas na Fig. 2.12. Ressalta-se que, embora as condições de contorno possam ser resumidas em dois desenhos esquemáticos, a diferença entre os cinco casos analisados é devida aos carregamentos distintos para cada um deles. Os carregamentos distintos para cada caso seguem as Eqs. (2.31).

Tabela 2.1: Condições de contorno em $x=0$ e $x=X_{1}$.

\begin{tabular}{|c|c|}
\hline Casos & Condições de Contorno em $x=0$ e $x=X_{1}$ \\
\hline 1) $m=n(1$ ou 3$)$ & $\chi_{1}^{(m n)}=0 ; \quad T_{31}=0 ; \quad \frac{\partial \psi^{(m n)}}{\partial x_{1}}=0$ \\
\hline 2) $m n=13($ ou 31) & $\chi_{3}^{(13)}=0 ; \quad T_{11}=0 ; \quad \psi^{(13)}=0$ \\
\hline 3) $m=1$ & $\Phi_{3}^{(1)}=0 ; \quad T_{11}=0 ; \quad R^{(1)}=0$ \\
\hline 4) $m=3$ & $\Phi_{1}^{(3)}=0 ; \quad T_{31}=0 ; \quad \frac{\partial R^{(3)}}{\partial x_{1}}=0$ \\
\hline
\end{tabular}

Tabela 2.2: Condições de contorno em $z=0$ e $z=Z_{3}$.

\begin{tabular}{|c|c|}
\hline Casos & Condições de Contorno em $z=0$ e $z=Z_{3}$ \\
\hline 1) $m=n(1$ ou 3$)$ & $\chi_{3}^{(m n)}=0 ; \quad T_{13}=0 ; \quad \psi^{(m n)}=0$ \\
\hline 2) $m n=13($ ou 31$)$ & $\chi_{1}^{(13)}=0 ; \quad T_{33}=0 ; \quad \frac{\partial \psi^{(13)}}{\partial z_{3}}=0$ \\
\hline 3) $m=1$ & $\Phi_{1}^{(1)}=0 ; \quad T_{33}=0 ; \quad \frac{\partial R^{(1)}}{\partial z_{3}}=0$ \\
\hline 4) $m=3$ & $\Phi_{3}^{(3)}=0 ; \quad T_{13}=0 ; \quad R^{(3)}=0$ \\
\hline
\end{tabular}




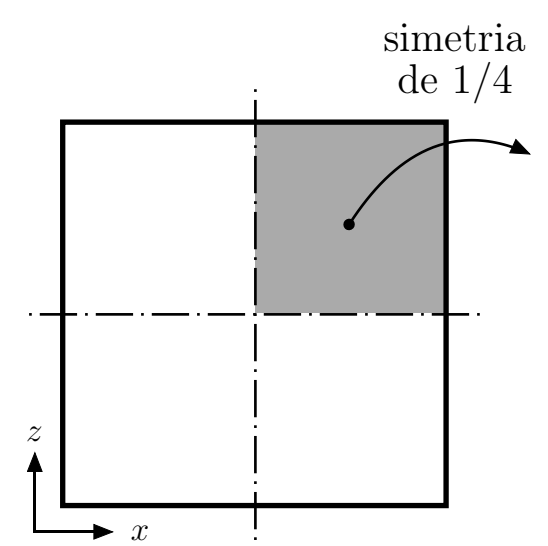

casos 1,2 e 5

casos 3 e 4

$(m n=11, m n=33$ ou $m=3)$ $(m n=13$ ou $m=1)$

célula unitária
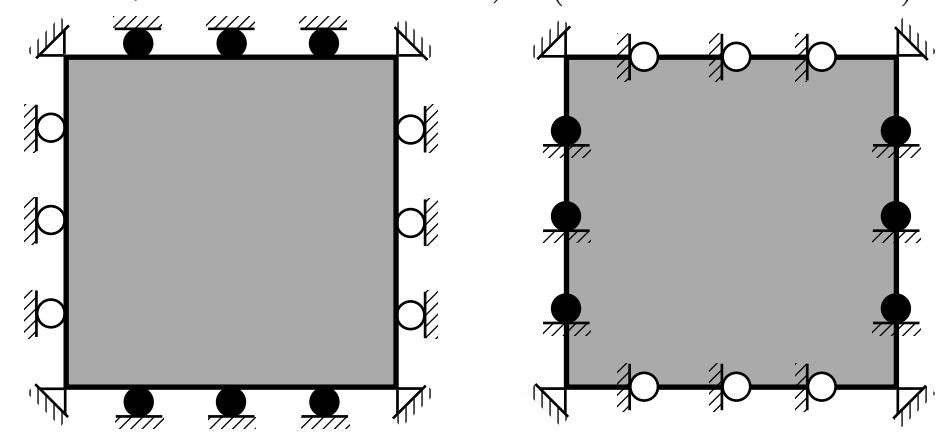

\begin{tabular}{|c|c|c|c|}
\hline $\begin{array}{c}\text { Graus de liberdade } \\
\text { prescritos }\end{array}$ & Aी & P & ก $~$
\end{tabular}

Figura 2.12: Condições de contorno para um quarto da célula unitária.

Os deslocamentos e potencial elétrico de algum ponto da célula deve ser prescrito a fim de evitar o problema de mais de uma solução; de outro modo, o problema será mal posto. A escolha do ponto para os valores prescritos não afeta os coeficientes homogeneizados, uma vez que para os seus cálculos utilizamos apenas derivadas das funções características (SILVA et al., 1999; SILVA; FONSECA; KIKUCHI, 1997; SILVA et al., 1999). Após resolver os deslocamentos e potenciais elétricos característicos, os coeficientes efetivos são calculados utilizando as Eq. (2.12), (2.13) e (2.14), as quais também são discretizadas em somas de $N$ elementos.

Para ilustrar os cinco casos de carregamento, toma-se como exemplo a distribuição de material mostrada na Tabela 2.3 e ilustrada na Fig. 2.13b. A escala de cinza presente ao redor do material 1 (representado em preto) é devido ao CAMD, pois o material é distribuído de forma contínua no interior dos elementos. As condições de contorno utilizadas neste exemplo estão mostradas na Fig. 2.12. Os carregamentos e funções características desse exemplo são mostrados nas Fig. 2.14 a 2.18. Para melhor visualização, esses carregamentos são mostrados apenas em 1/4 da célula unitária, já que nesse exemplo são aplicadas simetrias nos eixos $x$ e $z$.

Através das Eq. (2.30), monta-se as matrizes de rigidez $\mathbf{K}_{\mathbf{u u}}, \mathbf{K}_{\mathbf{u} \phi}$ e $\mathbf{K}_{\phi \phi}$ e os vetores de carregamento F e Q. Resolvendo os sistemas das Eq. (2.28) e (2.29), obtêm-se os valores nodais $\widehat{\boldsymbol{\chi}}, \widehat{\boldsymbol{\psi}}, \widehat{\boldsymbol{\Phi}}$ e $\widehat{\mathbf{R}}$. Em posse desses valores pode-se calcular as propriedades efetivas do material através das Eq. (C.36), (C.37) e (C.38). Os valores dos carregamentos 
Tabela 2.3: Exemplo: distribuição de material em uma malha de $7 \times 7$ elementos (ver Fig. 2.13b).

\begin{tabular}{|c||c|c|c|c|c|c|c|c|}
\hline Nó & 1 & 2 & 3 & 4 & 5 & 6 & 7 & 8 \\
\hline \hline 1 & 0 & 0 & 0 & 0 & 1 & 1 & 1 & 1 \\
\hline 2 & 0 & 0 & 0 & 0 & 1 & 1 & 1 & 1 \\
\hline 3 & 0 & 0 & 0 & 0 & 1 & 1 & 1 & 1 \\
\hline 4 & 0 & 0 & 0 & 1 & 1 & 1 & 1 & 1 \\
\hline 5 & 1 & 1 & 1 & 1 & 1 & 0 & 0 & 0 \\
\hline 6 & 1 & 1 & 1 & 1 & 0 & 0 & 0 & 0 \\
\hline 7 & 1 & 1 & 1 & 1 & 0 & 0 & 0 & 0 \\
\hline 8 & 1 & 1 & 1 & 1 & 0 & 0 & 0 & 0 \\
\hline
\end{tabular}

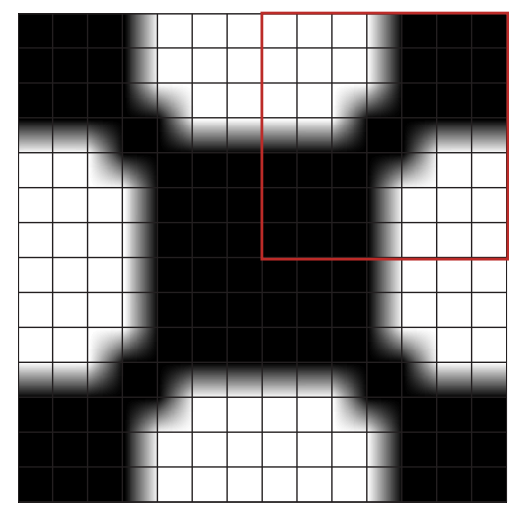

(a)

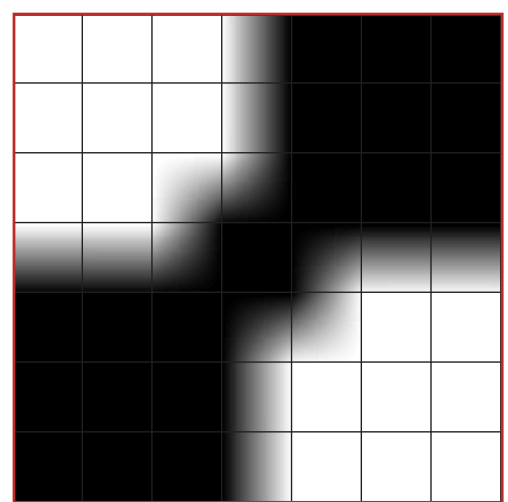

(b)

Figura 2.13: Exemplo de distribuição de material: (a) célula unitária e (b) domínio de projeto (1/4 da célula unitária); os valores de $\rho$ estão listados na Tabela 2.3.

$\mathbf{F}$ e $\mathbf{Q}$ e os valores nodais das funções características $\chi, \boldsymbol{\psi}, \boldsymbol{\Phi}$ e $\mathbf{R}$ para o exemplo da Fig. 2.13 são mostrados nas Fig. 2.14 a 2.18.

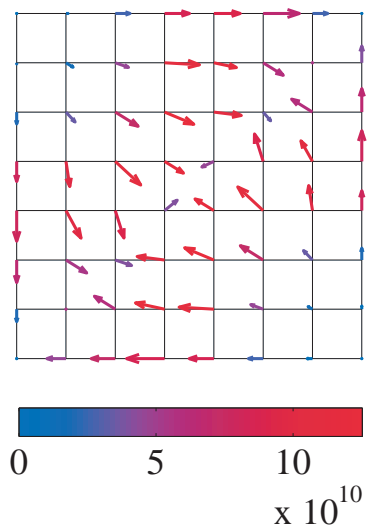

(a) $\mathbf{F}^{(11)}$
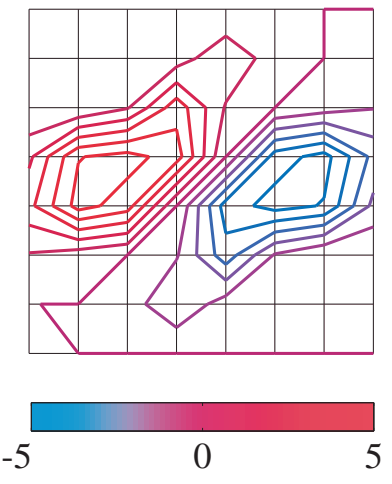

(b) $\mathbf{Q}^{(11)}$

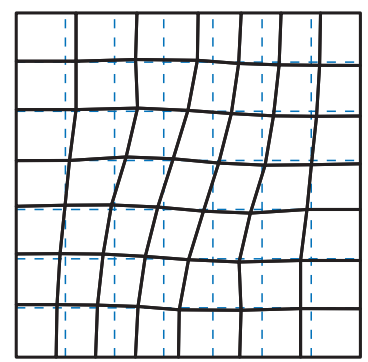

Valor máx.: $7.4 \mathrm{e}+000$

(c) $\chi^{(11)}$
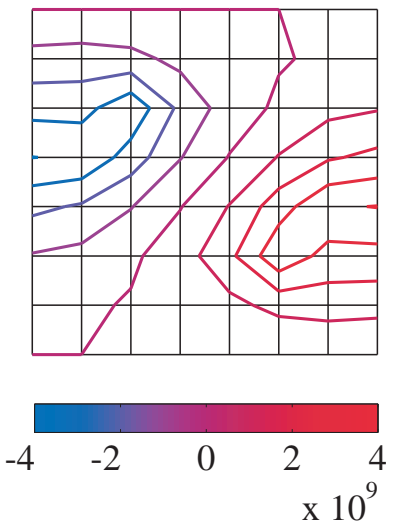

(d) $\psi^{(11)}$

Figura 2.14: Caso $1(m n=11)$ : carregamentos e funções características.

No Apêndice C mostra-se a verificação da implementação numérica do Método de Homogeneização. 


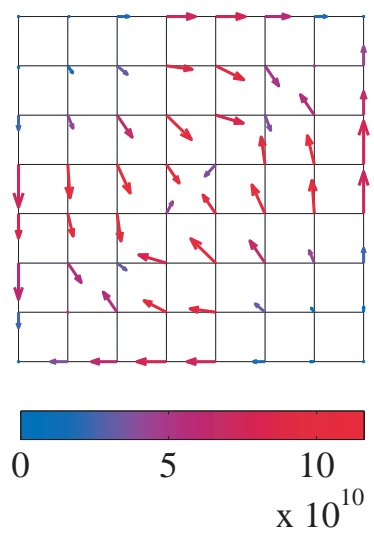

(a) $\mathbf{F}^{(33)}$
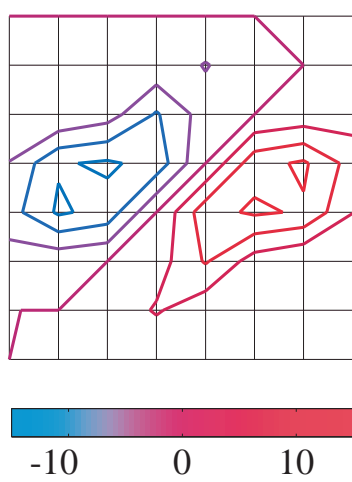

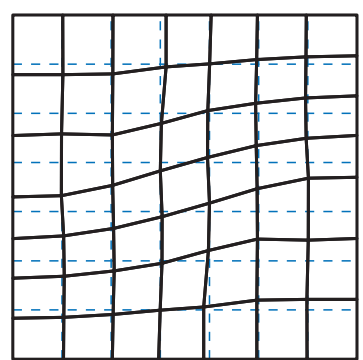

Valor máx.: $6.7 \mathrm{e}+000$
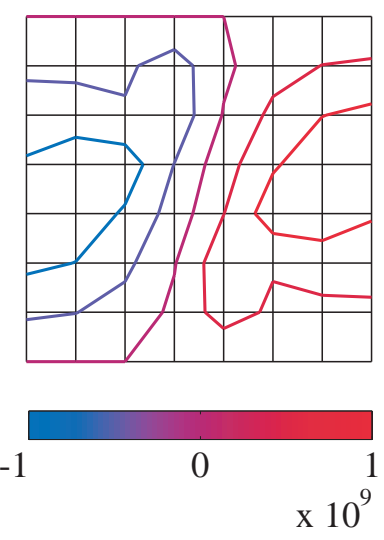

(d) $\boldsymbol{\psi}^{(33)}$

Figura 2.15: Caso $2(m n=33)$ : carregamentos e funções características.

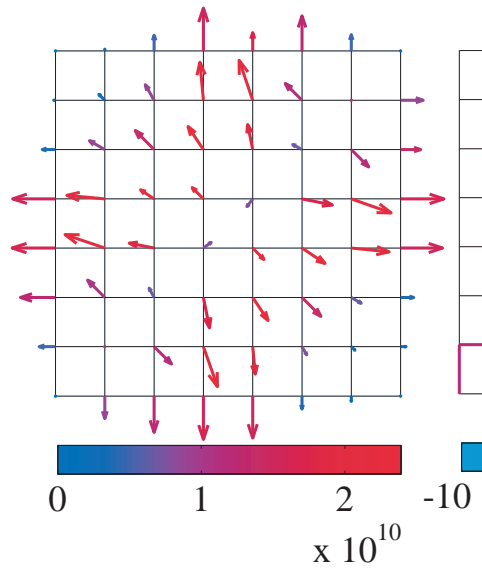

(a) $\mathbf{F}^{(13)}$
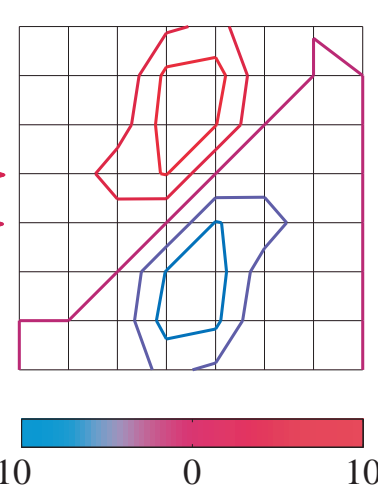

(b) $\mathbf{Q}^{(13)}$

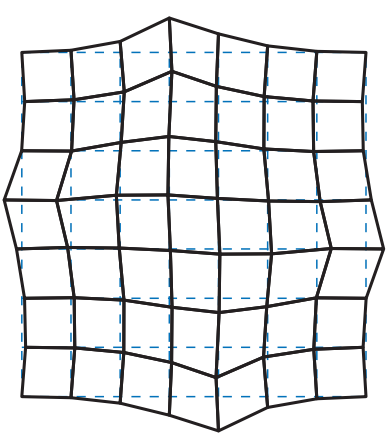

Valor máx.: $2.4 \mathrm{e}+000$

(c) $\chi^{(13)}$
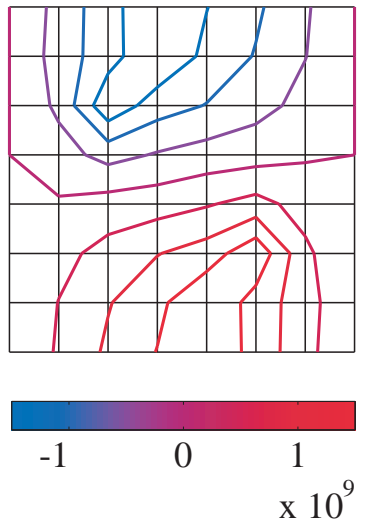

(d) $\boldsymbol{\psi}^{(13)}$

Figura 2.16: Caso $3(m n=13)$ : carregamentos e funções características.

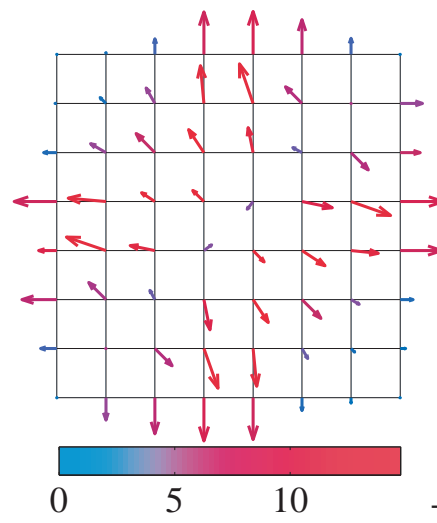

(a) $\mathbf{F}^{(1)}$

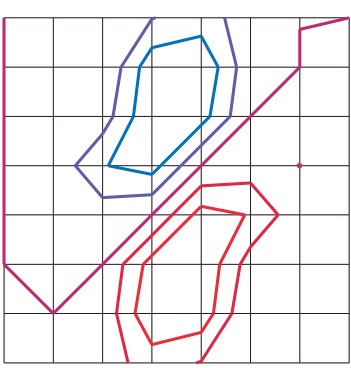

0

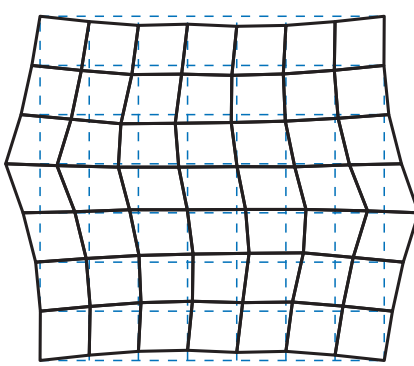

Valor máx.: 1.4e-009

(c) $\chi^{(1)}$
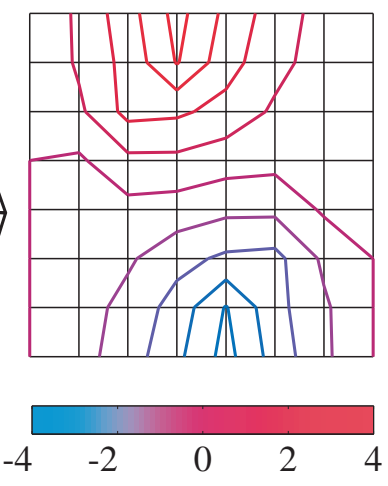

(d) $\boldsymbol{\psi}^{(1)}$

Figura 2.17: Caso $4(m=1)$ : carregamentos e funções características.

\subsubsection{Otimização de Material Discreto (OMD)}

A idéia central do OMD é basicamente uma extensão do MOT estrutural; porém, ao invés de escolher entre um sólido ou vazio, tem-se um conjunto de materiais com 


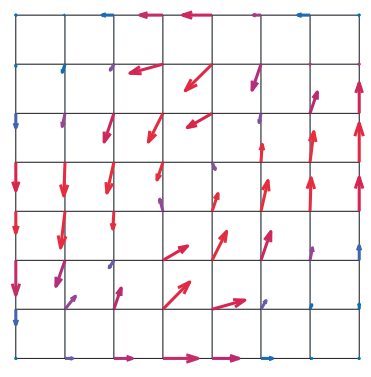

\begin{tabular}{llll}
\hline & 5 & 10 & 15
\end{tabular}
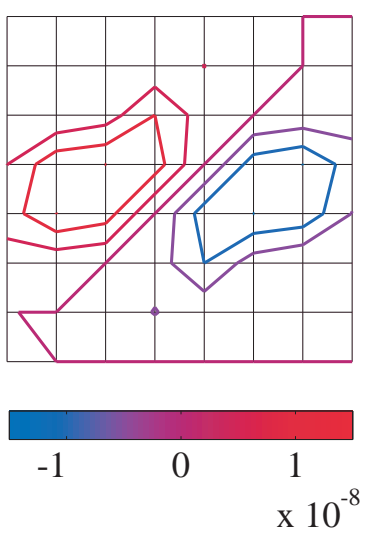

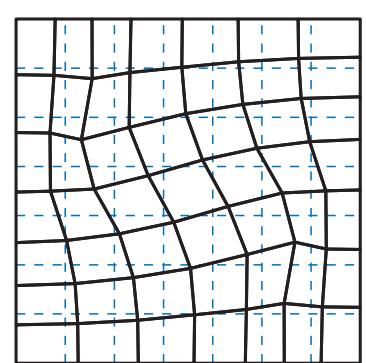

Valor máx.: 1.0e-009

(c) $\chi^{(3)}$

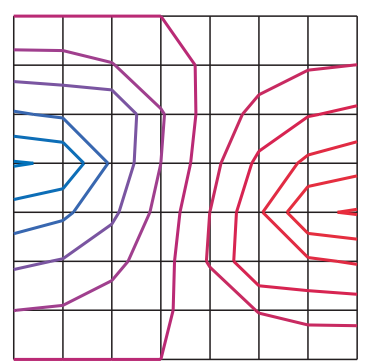

$-5$ 5

(d) $\boldsymbol{\psi}^{(3)}$

(a) $\mathbf{F}^{(3)}$

(b) $\mathbf{Q}^{(3)}$

Figura 2.18: Caso $5(m=3)$ : carregamentos e funções características.

propriedades distintas. Esta metodologia pode ser definida como: em cada posição (ou elemento) do domínio de projeto, encontre um material distinto a partir de um conjunto de materiais candidatos, de tal forma que a função objetivo seja maximizada. Para o caso de orientação da polarização de materiais piezelétricos, cada material candidato tem as propriedades calculadas num determinado ângulo. Assim, define-se uma quantidade pré-determinada de ângulos e cabe ao OMD indicar qual é o melhor material candidato (ver Fig. 2.19).

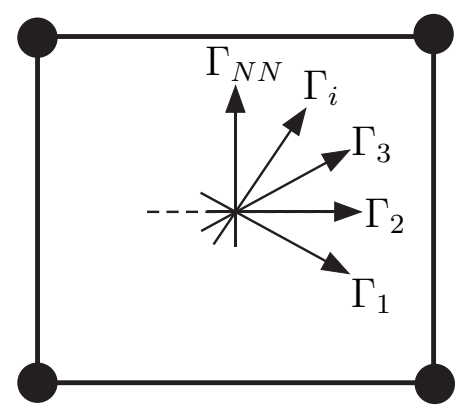

Figura 2.19: Ao invés de considerar as variáveis de projeto como contínuas, define-se $N N$ materiais candidatos a serem considerados na otimização.

Conforme a abordagem clássica do MOT, a parametrização do OMD é realizada no nível do elemento finito. O tensor piezelétrico do elemento, $\mathbf{e}^{e}$, é expresso como uma somatória ponderada dos tensores piezelétricos dos materiais candidatos, $\mathbf{e}_{i}$, conforme mostra a Eq. (2.32):

$$
\mathbf{e}_{m a t_{j}}^{e}=\sum_{i=1}^{n^{e}} w_{i} \mathbf{e}_{i}=w_{1} \mathbf{e}_{1}+w_{2} \mathbf{e}_{2}+\cdots+w_{n^{e}} \mathbf{e}_{n^{e}}, \quad 0 \leq w_{i} \leq 1
$$

onde $w_{i}$ são os fatores de peso, $n^{e}$ é o número de ângulos candidatos à polarização piezelétrica, $\mathbf{e}_{m a t_{j}}^{e}$ é o tensor piezelétrico resultante do elemento $e$ do material $j$, e $\mathbf{e}_{i}$ é o tensor piezelétrico rotacionado para o ângulo candidato $i$. Cada matriz constitutiva 
$\mathbf{e}_{i}$ pode ser calculada pela seguinte expressão:

$$
\mathbf{e}_{i}=\left(\mathbf{R}_{1}^{\theta_{i}}\right)^{t} \mathbf{e}_{m a t_{j}}^{0} \mathbf{R}_{2}^{\theta_{i}}
$$

e

$$
\mathbf{R}_{1}^{\theta_{i}}=\left[\begin{array}{ccc}
c^{2} & s^{2} & s c \\
s^{2} & c^{2} & -s c \\
-2 s c & 2 s c & c^{2}-s^{2}
\end{array}\right] \quad \text { e } \quad \mathbf{R}_{2}^{\theta_{i}}=\left[\begin{array}{cc}
c & s \\
-s & c
\end{array}\right]
$$

onde $\mathbf{e}_{\text {mat }_{j}}^{0}$ é o tensor piezelétrico original do material $j$ com direção positiva do eixo $z$, $s=\sin \left(\theta_{i}\right), c=\cos \left(\theta_{i}\right)$, e $\theta_{i}$ é o ângulo discreto adotado para cada material. Os índices $i$ e $j$ representam o número de ângulos candidatos e o material A ou B da Eq. (4.6), respectivamente.

Os fatores de peso $w_{i}$ da Eq. (2.32) devem assumir valores entre 0 e 1 , pois nenhum tensor piezelétrico pode contribuir com mais do que a propriedade piezelétrica do material e valores negativos não tem sentido físico. Semelhante à abordagem clássica do MOT, esses pesos agem como "chaveadores", ligando e desligando cada ângulo de polarização candidato, de tal forma a maximizar a função objetivo. No início do processo de otimização, o tensor $\mathbf{e}^{e}$ é formado por contribuições de vários ângulos de polarização candidatos. Entretanto, ao final do processo, apenas um ângulo candidato deve se sobressair (STEGMANN; LUND, 2005). Assim, o OMD deve assegurar que o otimizador seja capaz de conduzir os fatores de peso à zero e apenas um seja unitário. Para isso, mais variáveis de projeto são necessárias para representar o ângulo candidato, denominadas por $\gamma_{i}^{e}$, que podem assumir valores contínuos entre 0 e 1 . As funções de peso são calculadas por:

$$
w_{i}=\frac{\hat{w}_{i}}{\sum_{k=1}^{n^{e}} \hat{w}_{k}}, \quad \text { onde } \quad \hat{w}_{i}=\left(\gamma_{i}^{e}\right)^{p_{\gamma}} \prod_{j=1, j \neq i}^{n^{e}}\left(1-\left(\gamma_{i}^{e}\right)^{p_{\gamma}}\right)
$$

Para conduzir os valores de $\gamma_{i}^{e}$ para 0 ou 1, é implementada uma técnica de penalização utilizando o expoente $p_{\gamma}$, o qual penaliza valores intermediários de $\gamma_{i}^{e}$. Além disso, o termo $\left(1-\left(\gamma_{i}^{e}\right)^{p_{\gamma}}\right)_{j \neq i}$ é introduzido de forma que um aumento de $\gamma_{i}^{e}$ resulta em uma diminuição de todos as outras funções de peso do mesmo elemento $e$. Os pesos são normalizados de forma a satisfazer a restrição que a soma das funções de peso seja unitária. Nota-se também que a Eq. (2.35) necessita apenas de restrições de caixa das variáveis de projeto $\gamma_{i}^{e}$, eliminando assim a adição de restrições complexas no problema de otimização. Esta formulação conduz o problema a um maior número de variáveis de projeto; porém, desse modo é possível reduzir soluções de ótimos locais (STEGMANN; LUND, 2005). 


\subsubsection{Controle de Gradação de Material}

A formulação CAMD garante a distribuição contínua de material nos elementos finitos; entretanto, esta formulação não fornece um controle de gradação do material. Para obter o controle de gradação do material e garantir independência de malha, neste trabalho aplica-se a formulação CAMD (CARBONARI; SILVA; PAULINO, 2007) em conjunto com a técnica de projeção baseada no trabalho de Bruns e Tortorelli (BRUNS; TORTORELLI, 2001). Esta técnica adiciona uma nova camada de pseudo-densidades nodais de material $\left(d_{n}\right)$ sobreposta a atual camada de variáveis de projeto nodais $\left(\rho_{n}\right)$. Para cada variável de projeto $\rho_{i}$ define-se uma área circular de raio $r_{\text {grad }}$, cujo centro é a própria posição do nó $i$, conforme mostrado na Fig. 2.20. Os valores de $\rho_{i}$ passam a ser em função dos valores das pseudo-densidades pertencentes a esta área circular e às distâncias entre cada pseudo-densidade e o centro da área.
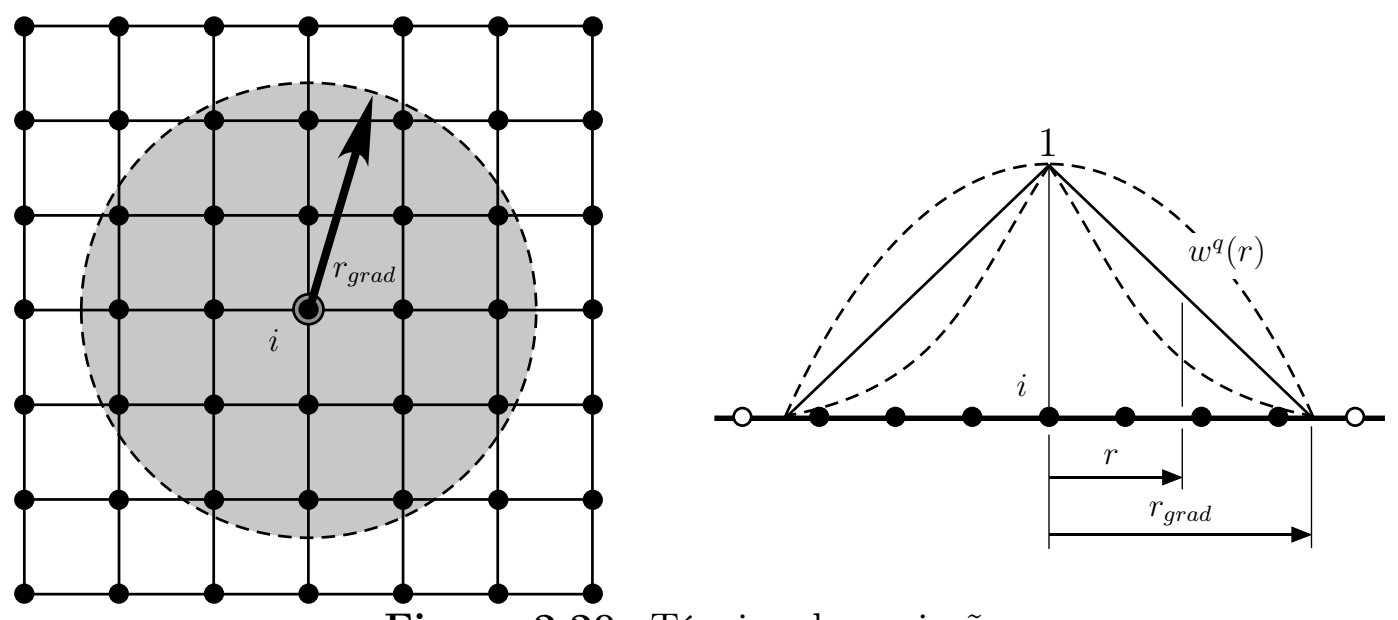

Figura 2.20: Técnica de projeção.

Assim:

$$
\rho_{n}=f\left(d_{n}\right)
$$

onde

$$
\rho_{i}=f\left(d_{j}\right)=\frac{\sum_{j \in S_{i}} d_{j} w^{q}\left(r_{i j}\right)}{\sum_{j \in S_{i}} w^{q}\left(r_{i j}\right)}
$$

e $r_{i j}$ é a distância entre os nós $i$ e $j$

$$
r_{i j}=\left\|\mathbf{x}_{j}-\mathbf{x}_{i}\right\|
$$

e $S_{i}$ é o sub-conjunto dos nós no interior da área circular. O peso do multiplicador $w$ é definido por: 


$$
w^{q}\left(r_{i j}\right)=\left\{\begin{array}{cr}
\left(\frac{r_{\text {grad }}-r_{i j}}{r_{\text {grad }}}\right)^{q}, & \text { se } \mathbf{x}_{j} \in S_{i} \\
0, & \text { caso contrário }
\end{array}\right.
$$

e o coeficiente $q$ pode ser utilizado para relações não-lineares, se for adotado como diferente de 1.

A sensibilidade em relação às variáveis de projeto são obtidas utilizando a regra da cadeia:

$$
\frac{\partial(.)}{\partial d_{i}}=\sum_{j \in \Omega} \frac{\partial(.)}{\partial \rho_{j}} \frac{\partial \rho_{j}}{\partial d_{i}}
$$

onde $\Omega$ é o domínio de projeto e $\partial \rho_{j} / \partial d_{i}$ é não-nulo apenas nos nós $j$ pertencentes a $S_{i}$ referente ao nó $i$. Além disso,

$$
\frac{\partial \rho_{j}}{\partial d_{i}}=\frac{w^{q}\left(r_{i j}\right)}{\sum_{k \in S_{j}} w^{q}\left(r_{k j}\right)}
$$

onde $\partial(.) / \partial \rho_{j}$ é calculado utilizando métodos tradicionais, tais como o método adjunto, por exemplo.

\subsubsection{Análise de Sensibilidade}

A análise de sensibilidade fornece os gradientes das propriedades homogeneizadas, os quais permitem que o programa de otimização defina as direções que serão tomadas para cada variável de projeto. Para o cálculo dos gradientes é aplicado o método adjunto. O cálculo das sensibilidades parte da derivação das Eq. (C.30), (C.31) e (C.32) em relação às variáveis de projeto $\rho_{n}$. Para facilitar os cálculos das sensibilidades, pode-se reescrever as Eq. (2.28) e (2.29) na forma de um único sistema:

$$
\left[\begin{array}{cc}
\mathbf{K}_{\mathbf{u u}} & \mathbf{K}_{\mathbf{u} \varphi} \\
\mathbf{K}_{\mathbf{u} \varphi}^{t} & -\mathbf{K}_{\varphi \varphi}
\end{array}\right]\left[\begin{array}{ll}
\boldsymbol{\chi}^{(m n)} & \boldsymbol{\Phi}^{(m)} \\
\boldsymbol{\Psi}^{(m n)} & \mathbf{R}^{(m)}
\end{array}\right]=\left[\begin{array}{ll}
\mathbf{F}^{(m n)} & \mathbf{F}^{(m)} \\
\mathbf{Q}^{(m n)} & \mathbf{Q}^{(m)}
\end{array}\right] \Leftrightarrow \mathbf{K X}=\mathbf{Y}
$$

Derivando este sistema de equações, tem-se que:

$$
\frac{\partial \mathbf{K}}{\partial \rho_{i}} \mathbf{X}+\mathbf{K} \frac{\partial \mathbf{X}}{\partial \rho_{i}}=\frac{\partial \mathbf{Y}}{\partial \rho_{i}} \Leftrightarrow \frac{\partial \mathbf{X}}{\partial \rho_{i}}=\mathbf{K}^{-1} \mathbf{M}, \quad \text { onde } \quad \mathbf{M}=\frac{\partial \mathbf{Y}}{\partial \rho_{i}}-\frac{\partial \mathbf{K}}{\partial \rho_{i}} \mathbf{X}
$$

Para cada elemento finito, tem-se que: 


$$
\begin{aligned}
& \left\{\frac{\partial \boldsymbol{\chi}^{(m n)}}{\partial \rho_{i}}\right\}_{e}=\mathbf{H}_{e m}^{t} \mathbf{K}^{-1} \mathbf{M}^{(m n)} \Rightarrow\left\{\frac{\partial \boldsymbol{\chi}^{(m n)}}{\partial \rho_{i}}\right\}_{e}^{t}=\left(\mathbf{M}^{(m n)}\right)^{t} \mathbf{K}^{-1} \mathbf{H}_{e m} \\
& \left\{\frac{\partial \boldsymbol{\psi}^{(m n)}}{\partial \rho_{i}}\right\}_{e}=\mathbf{H}_{e l}^{t} \mathbf{K}^{-1} \mathbf{M}^{(m n)} \Rightarrow\left\{\frac{\partial \boldsymbol{\psi}^{(m n)}}{\partial \rho_{i}}\right\}_{e}^{t}=\left(\mathbf{M}^{(m n)}\right)^{t} \mathbf{K}^{-1} \mathbf{H}_{e l} \\
& \left\{\frac{\partial \boldsymbol{\Phi}^{(m)}}{\partial \rho_{i}}\right\}_{e}=\mathbf{H}_{e l}^{t} \mathbf{K}^{-1} \mathbf{M}^{(m)} \Rightarrow\left\{\frac{\partial \boldsymbol{\Phi}^{(m)}}{\partial \rho_{i}}\right\}_{e}^{t}=\left(\mathbf{M}^{(m)}\right)^{t} \mathbf{K}^{-1} \mathbf{H}_{e l} \\
& \left\{\frac{\partial \mathbf{R}^{(m)}}{\partial \rho_{i}}\right\}_{e}=\mathbf{H}_{e l}^{t} \mathbf{K}^{-1} \mathbf{M}^{(m)} \Rightarrow\left\{\frac{\partial \mathbf{R}^{(m)}}{\partial \rho_{i}}\right\}_{e}^{t}=\left(\mathbf{M}^{(m)}\right)^{t} \mathbf{K}^{-1} \mathbf{H}_{e l}
\end{aligned}
$$

onde $\mathbf{H}_{e m}$ é uma matriz $N M \times 5$ preenchida de 1 nos graus de liberdade mecânicos do elemento e 0 para todos os demais componentes; e $\mathbf{H}_{e l}$ é uma matriz $N E \times 5$ preenchida de 1 nos graus de liberdade elétricos do elemento e 0 para todos os demais componentes. $N M$ e $N E$ são, respectivamente, os números de graus de liberdade mecânicos e elétricos de todo o domínio.

A seguir, são apresentadas separadamente as equações para cada propriedade, em conjunto com o método adjunto aplicado em cada uma delas.

\section{- Derivada do tensor elástico (c)}

A derivada da Eq. (C.30) é dada por (SILVA et al., 1999):

$$
\begin{aligned}
\frac{\partial \mathbf{c}_{H}}{\partial \rho_{i}} & =\frac{1}{|Y|}\left\{\int _ { Y } \left[\left(\mathbf{I}+\partial_{\mathbf{y}} \boldsymbol{\chi}(\mathbf{x}, \mathbf{y})\right): \frac{\partial \mathbf{c}(\mathbf{x}, \mathbf{y})}{\partial \rho_{i}}:\left(\mathbf{I}+\partial_{\mathbf{y}} \boldsymbol{\chi}(\mathbf{x}, \mathbf{y})\right)+\right.\right. \\
& +\left(\mathbf{I}+\partial_{\mathbf{y}} \boldsymbol{\chi}(\mathbf{x}, \mathbf{y})\right): \frac{\partial \mathbf{e}(\mathbf{x}, \mathbf{y})}{\partial \rho_{i}} \nabla_{\mathbf{y}} \boldsymbol{\psi}(\mathbf{x}, \mathbf{y})+ \\
& +\frac{\partial\left(\partial_{\mathbf{y}} \boldsymbol{\chi}(\mathbf{x}, \mathbf{y})\right)}{\partial \rho_{i}}: \mathbf{c}(\mathbf{x}, \mathbf{y}):\left(\mathbf{I}+\partial_{\mathbf{y}} \boldsymbol{\chi}(\mathbf{x}, \mathbf{y})\right)+ \\
& \left.\left.+\left(\mathbf{I}+\partial_{\mathbf{y}} \boldsymbol{\chi}(\mathbf{x}, \mathbf{y})\right): \mathbf{e}(\mathbf{x}, \mathbf{y}) \frac{\partial\left(\nabla_{\mathbf{y}} \boldsymbol{\psi}(\mathbf{x}, \mathbf{y})\right)}{\partial \rho_{i}}\right] d Y\right\}
\end{aligned}
$$

ou, na forma matricial:

$$
\begin{aligned}
\frac{\partial \mathbf{c}_{H}}{\partial \rho_{i}}=\sum_{e} & \left\{(\mathbf{I}+\boldsymbol{\chi})_{e}^{t}\left(\frac{\partial \mathbf{K}_{\mathbf{u u}}}{\partial \rho_{i}}\right)_{e}(\mathbf{I}+\boldsymbol{\chi})_{e}+(\mathbf{I}+\boldsymbol{\chi})_{e}^{t}\left(\frac{\partial \mathbf{K}_{\mathbf{u} \phi}}{\partial \rho_{i}}\right)_{e}\left(\boldsymbol{\psi}^{(m n)}\right)_{e}+\right. \\
& \left.+\left\{\frac{\partial \boldsymbol{\chi}^{(m n)}}{\partial \rho_{i}}\right\}_{e}^{t}\left(\mathbf{K}_{\mathbf{u u}}\right)_{e}(\mathbf{I}+\boldsymbol{\chi})_{e}+(\mathbf{I}+\boldsymbol{\chi})_{e}^{t}\left(\mathbf{K}_{\mathbf{u} \phi}\right)_{e}\left(\frac{\partial \boldsymbol{\psi}^{(m n)}}{\partial \rho_{i}}\right)_{e}\right\}
\end{aligned}
$$

Os termos desconhecidos são $\partial \mathbf{c}(\mathbf{x}, \mathbf{y}) / \partial \rho_{i}, \quad \partial \mathbf{e}(\mathbf{x}, \mathbf{y}) / \partial \rho_{i}, \quad \partial\left(\partial_{\mathbf{y}} \boldsymbol{\chi}(\mathbf{x}, \mathbf{y})\right) / \partial \rho_{i} \quad \mathrm{e}$ $\partial\left(\nabla_{\mathbf{y}} \psi(\mathbf{x}, \mathbf{y})\right) / \partial \rho_{i}$. Para calcular $\partial \mathbf{c}(\mathbf{x}, \mathbf{y}) / \partial \rho_{i}$ tem-se que: 


$$
\begin{gathered}
\mathbf{c}=\rho \mathbf{c}_{1}+(1-\rho) \mathbf{c}_{2} \Rightarrow \frac{\partial \mathbf{c}}{\partial \rho}=\mathbf{c}_{1}-\mathbf{c}_{2} \\
\rho=\sum_{i=1}^{4} \rho_{i}^{p} N_{i} \Rightarrow \frac{\partial \rho}{\partial \rho_{i}}=p \rho_{i}^{p-1} N_{i}
\end{gathered}
$$

e, pela regra da cadeia, vem:

$$
\frac{\partial \mathbf{c}}{\partial \rho_{i}}=\frac{\partial \mathbf{c}}{\partial \rho} \frac{\partial \rho}{\partial \rho_{i}} \Rightarrow \frac{\partial \mathbf{c}}{\partial \rho_{i}}=\left(\mathbf{c}_{1}-\mathbf{c}_{2}\right) p \rho_{i}^{p-1} N_{i}
$$

Para calcular $\partial \mathbf{e}(\mathbf{x}, \mathbf{y}) / \partial \rho_{i}$, tem-se que:

$$
\mathbf{e}=\rho \mathbf{e}_{1}+(1-\rho) \mathbf{e}_{2} \Rightarrow \frac{\partial \mathbf{e}}{\partial \rho}=\mathbf{e}_{1}-\mathbf{e}_{2}
$$

e, pela regra da cadeia, vem:

$$
\frac{\partial \mathbf{e}}{\partial \rho_{i}}=\frac{\partial \mathbf{e}}{\partial \rho} \frac{\partial \rho}{\partial \rho_{i}} \Rightarrow \frac{\partial \mathbf{e}}{\partial \rho_{i}}=\left(\mathbf{e}_{1}-\mathbf{e}_{2}\right) p \rho_{i}^{p-1} N_{i}
$$

O cálculo das Eq. (2.52) e (2.54) depende apenas dos nós dos elementos vizinhos, pois os termos $\partial \mathbf{c} / \partial \rho$ e $\partial \mathbf{e} / \partial \rho$ são nulos para os demais elementos. Portanto, estas equações têm um custo computacional baixo.

O cálculo explícito dos termos $\partial\left(\partial_{\mathbf{y}} \chi(\mathbf{x}, \mathbf{y})\right) / \partial \rho_{i}$ e $\partial\left(\nabla_{\mathbf{y}} \psi(\mathbf{x}, \mathbf{y})\right) / \partial \rho_{i}$ gera um custo computacional alto, pois dependem de todas as variáveis do domínio de projeto. Para contornar este problema também utiliza-se o método adjunto. Assim, a terceira parcela da Eq.(2.49) pode ser reescrita por:

$$
\left\{\frac{\partial \boldsymbol{\chi}^{(m n)}}{\partial \rho_{i}}\right\}_{e}^{t}\left(\mathbf{K}_{\mathbf{u u}}\right)_{e}(\mathbf{I}+\boldsymbol{\chi})_{e}=\left(\mathbf{M}^{(m n)}\right)^{t} \mathrm{I}_{e}
$$

Utilizando a Eq. (2.44), tem-se que:

$$
\begin{aligned}
\left(\mathbf{M}^{(m n)}\right)^{t} \mathbf{K}^{-1} \mathbf{H}_{e m}\left(\mathbf{K}_{\mathbf{u u}}\right)_{e}(\mathbf{I}+\boldsymbol{\chi})_{e} & =\left(\mathbf{M}^{(m n)}\right)^{t} \mathbf{I}_{e} \\
\mathbf{K} \mathbf{I}_{e} & =\mathbf{J}_{e}
\end{aligned}
$$

onde $\mathbf{J}_{e}=\mathbf{H}_{e m}\left(\mathbf{K}_{\mathbf{u u}}\right)_{e}(\mathbf{I}+\boldsymbol{\chi})_{e}$. Obtendo-se a matriz $\mathbf{I}_{e}$, pode-se calcular a Eq. (2.55).

De modo análogo, pode-se impor que a quarta parcela da Eq. (2.49) seja igual a: 


$$
\left(\mathbf{I}+\boldsymbol{\chi}^{(m n)}\right)_{e}^{t}\left(\mathbf{K}_{\mathbf{u} \varphi}\right)_{e}\left\{\frac{\partial \boldsymbol{\psi}^{(m n)}}{\partial \mathbf{x}}\right\}_{e}=\mathbf{P}_{e}^{t} \mathbf{M}^{(m n)}
$$

Utilizando a Eq. (2.45), tem-se que:

$$
\begin{aligned}
(\mathbf{I}+\boldsymbol{\chi})_{e}^{t}\left(\mathbf{K}_{\mathbf{u} \varphi)_{e}} \mathbf{H}_{e l}^{t} \mathbf{K}^{-1} \mathbf{M}^{(m n)}\right. & =\mathbf{P}_{e}^{t} \mathbf{M}^{(m n)} \\
\left(\mathbf{M}^{(m n)}\right)^{t} \mathbf{K}^{-1} \mathbf{V}_{e} & =\left(\mathbf{M}^{(m n)}\right)^{t} \mathbf{P}_{e} \\
\mathbf{K} \mathbf{P}_{e} & =\mathbf{V}_{e}
\end{aligned}
$$

onde $\mathbf{V}_{e}=(\mathbf{I}+\boldsymbol{\chi})_{e}^{t}\left(\mathbf{K}_{\mathbf{u} \varphi}\right)_{e} \mathbf{H}_{e l}^{t}$. Obtendo-se $\mathbf{P}_{e}$, pode-se calcular a Eq. (2.57).

\section{- Derivada do tensor piezelétrico (e)}

A derivada da Eq. (C.31) é dada por (SILVA et al., 1999):

$$
\begin{aligned}
\frac{\partial \mathbf{e}_{H}}{\partial \rho_{i}} & =\frac{1}{|Y|}\left\{\int _ { Y } \left[\left(\mathbf{I}+\partial_{\mathbf{y}} \boldsymbol{\chi}(\mathbf{x}, \mathbf{y})\right): \frac{\partial \mathbf{e}(\mathbf{x}, \mathbf{y})}{\partial \rho_{i}}\left(\mathbf{I}+\nabla_{\mathbf{y}} \mathbf{R}(\mathbf{x}, \mathbf{y})\right)-\right.\right. \\
& -\left(\mathbf{I}+\nabla_{\mathbf{y}} \mathbf{R}(\mathbf{x}, \mathbf{y})\right) \frac{\partial \boldsymbol{\varepsilon}^{S}(\mathbf{x}, \mathbf{y})}{\partial \rho_{i}} \nabla_{\mathbf{y}} \boldsymbol{\psi}(\mathbf{x}, \mathbf{y})+ \\
& +\frac{\partial\left(\partial_{\mathbf{y}} \boldsymbol{\chi}(\mathbf{x}, \mathbf{y})\right)}{\partial \rho_{i}}: \mathbf{e}(\mathbf{x}, \mathbf{y})\left(\mathbf{I}+\nabla_{\mathbf{y}} \mathbf{R}(\mathbf{x}, \mathbf{y})\right)- \\
& \left.\left.-\left(\mathbf{I}+\nabla_{\mathbf{y}} \mathbf{R}(\mathbf{x}, \mathbf{y})\right) \boldsymbol{\varepsilon}^{S}(\mathbf{x}, \mathbf{y}) \frac{\partial\left(\nabla_{\mathbf{y}} \boldsymbol{\psi}(\mathbf{x}, \mathbf{y})\right)}{\partial \rho_{i}}\right] d Y\right\}
\end{aligned}
$$

ou, na forma matricial:

$$
\begin{aligned}
\frac{\partial \mathbf{e}_{H}}{\partial \rho_{i}}=\sum_{e} & \left\{(\mathbf{I}+\boldsymbol{\chi})_{e}^{t}\left(\frac{\partial \mathbf{K}_{\mathbf{u} \phi}}{\partial \rho_{i}}\right)_{e}\left(\mathbf{I}+\mathbf{R}^{(m)}\right)_{e}+\left(\mathbf{I}+\mathbf{R}^{(m)}\right)_{e}^{t}\left(\frac{\partial \mathbf{K}_{\phi \phi}}{\partial \rho_{i}}\right)_{e}\left(\boldsymbol{\psi}^{(m n)}\right)_{e}+\right. \\
& \left.+\left\{\frac{\partial \boldsymbol{\chi}^{(m n)}}{\partial \rho_{i}}\right\}_{e}^{t}\left(\mathbf{K}_{\mathbf{u} \phi}\right)_{e}\left(\mathbf{I}+\mathbf{R}^{(m)}\right)_{e}+\left(\mathbf{I}+\mathbf{R}^{(m)}\right)_{e}^{t}\left(\mathbf{K}_{\phi \phi}\right)_{e}\left(\frac{\partial \boldsymbol{\psi}^{(m n)}}{\partial \rho_{i}}\right)_{e}\right\}(2.6)
\end{aligned}
$$

O termo $\partial \mathbf{e}(\mathbf{x}, \mathbf{y}) / \partial \rho_{i}$ pode ser visto na Eq. (2.54). Os termos desconhecidos são $\partial \varepsilon^{S}(\mathbf{x}, \mathbf{y}) / \partial \rho_{i}, \partial\left(\partial_{\mathbf{y}} \boldsymbol{\chi}(\mathbf{x}, \mathbf{y})\right) / \partial \rho_{i}$ e $\partial\left(\nabla_{\mathbf{y}} \boldsymbol{\psi}(\mathbf{x}, \mathbf{y})\right) / \partial \rho_{i}$. Para calcular $\partial \varepsilon^{S}(\mathbf{x}, \mathbf{y}) / \partial \rho_{i}$ tem-se que:

$$
\varepsilon=\rho \varepsilon_{1}+(1-\rho) \varepsilon_{2} \Rightarrow \frac{\partial \varepsilon}{\partial \rho}=\varepsilon_{1}-\varepsilon_{2}
$$

Pela regra da cadeia, vem:

$$
\frac{\partial \varepsilon}{\partial \rho_{i}}=\frac{\partial \varepsilon}{\partial \rho} \frac{\partial \rho}{\partial \rho_{i}} \Rightarrow \frac{\partial \varepsilon}{\partial \rho_{i}}=\left(\varepsilon_{1}-\varepsilon_{2}\right) p \rho_{i}^{p-1} N_{i}
$$


Analogamente às Eqs. (2.52) e (2.54), o custo computacional da Eq. (2.62) é baixo. Novamente, ao invés de se calcular explicitamente os termos $\partial\left(\partial_{\mathbf{y}} \boldsymbol{\chi}(\mathbf{x}, \mathbf{y})\right) / \partial \rho_{i}$ e $\partial\left(\nabla_{\mathbf{y}} \boldsymbol{\psi}(\mathbf{x}, \mathbf{y})\right) / \partial \rho_{i}$, calcula-se a terceira e quarta parcela da Eq. (2.59) pelo método adjunto. Assim, reescrevendo a terceira parcela da Eq. (2.60), tem-se que:

$$
\left\{\frac{\partial \boldsymbol{\chi}^{(m n)}}{\partial \rho_{i}}\right\}_{e}^{t}\left(\mathbf{K}_{\mathbf{u} \varphi}\right)_{e}\left(\mathbf{I}+\mathbf{R}^{(m)}\right)_{e}=\left(\mathbf{M}^{(m n)}\right)^{t} \mathbf{A}_{e}
$$

Utilizando a Eq. (2.45), tem-se que:

$$
\begin{aligned}
\left(\mathbf{M}^{(m n)}\right)^{t} \mathbf{K}^{-1} \mathbf{H}_{e m}\left(\mathbf{K}_{\mathbf{u} \varphi)_{e}}\left(\mathbf{I}+\mathbf{R}^{(m)}\right)_{e}\right. & =\left(\mathbf{M}^{(m n)}\right)^{t} \mathbf{A}_{e} \\
\mathbf{K} \mathbf{A}_{e} & =\mathbf{W}_{e}
\end{aligned}
$$

onde $\mathbf{W}_{e}=\mathbf{H}_{e l}\left(\mathbf{K}_{\mathbf{u} \varphi}\right)_{e}\left(\mathbf{I}+\mathbf{R}^{(m)}\right)_{e}$. Obtendo-se $\mathbf{A}_{e}$, pode-se calcular a Eq. (2.63).

Já a quarta parcela da Eq. (2.60) pode ser reescrita por:

$$
\left(\mathbf{I}+\mathbf{R}^{(m)}\right)_{e}^{t}\left(\mathbf{K}_{\varphi \varphi}\right)_{e}\left\{\frac{\partial \boldsymbol{\psi}^{(m n)}}{\partial \mathbf{x}}\right\}_{e}=\mathbf{G}_{e}^{t} \mathbf{M}^{(m n)}
$$

Utilizando a eq. (2.47), tem-se que:

$$
\begin{aligned}
\left(\mathbf{I}+\mathbf{R}^{(m)}\right)_{e}^{t}\left(\mathbf{K}_{\varphi \varphi}\right)_{e} \mathbf{H}_{e l}^{t} \mathbf{K}^{-1} \mathbf{M}^{(m n)} & =\mathbf{G}_{e}^{t} \mathbf{M}^{(m n)} \\
\left(\mathbf{M}^{(m n)}\right)^{t} \mathbf{K}^{-1} \mathbf{Z}_{e} & =\left(\mathbf{M}^{(m n)}\right)^{t} \mathbf{G}_{e} \\
\mathbf{K} \mathbf{G}_{e} & =\mathbf{Z}_{e}
\end{aligned}
$$

onde $\mathbf{Z}_{e}^{t}=\left(\mathbf{I}+\mathbf{R}^{(m)}\right)_{e}^{t}\left(\mathbf{K}_{\varphi \varphi}\right)_{e} \mathbf{H}_{e l}^{t}$. Obtendo-se $\mathbf{G}_{e}$, pode-se calcular a Eq. (2.65).

\section{- Derivada do tensor dielétrico $(\varepsilon)$}

A derivada da Eq. (C.32) é dada por (SILVA et al., 1999):

$$
\begin{aligned}
\frac{\partial \varepsilon_{H}}{\partial \rho_{i}} & =\frac{1}{|Y|}\left\{\int _ { Y } \left[\left(\mathbf{I}+\nabla_{\mathbf{y}} \mathbf{R}(\mathbf{x}, \mathbf{y})\right): \frac{\partial \boldsymbol{\varepsilon}^{S}(\mathbf{x}, \mathbf{y})}{\partial \rho_{i}}\left(\mathbf{I}+\nabla_{\mathbf{y}} \mathbf{R}(\mathbf{x}, \mathbf{y})\right)-\right.\right. \\
& -\partial_{\mathbf{y}} \mathbf{\Phi}(\mathbf{x}, \mathbf{y}): \frac{\partial \mathbf{e}(\mathbf{x}, \mathbf{y})}{\partial \rho_{i}}\left(\mathbf{I}+\nabla_{\mathbf{y}} \mathbf{R}(\mathbf{x}, \mathbf{y})\right)+ \\
& +\frac{\partial\left(\nabla_{\mathbf{y}} \mathbf{R}(\mathbf{x}, \mathbf{y})\right)}{\partial \rho_{i}}: \boldsymbol{\varepsilon}^{S}(\mathbf{x}, \mathbf{y})\left(\mathbf{I}+\nabla_{\mathbf{y}} \mathbf{R}(\mathbf{x}, \mathbf{y})\right)- \\
& \left.\left.-\frac{\partial\left(\partial_{\mathbf{y}} \mathbf{\Phi}(\mathbf{x}, \mathbf{y})\right)}{\partial \rho_{i}}: \mathbf{e}(\mathbf{x}, \mathbf{y})\left(\mathbf{I}+\nabla_{\mathbf{y}} \mathbf{R}(\mathbf{x}, \mathbf{y})\right)\right] d Y\right\}
\end{aligned}
$$


ou, na forma matricial:

$$
\begin{aligned}
\frac{\partial \boldsymbol{\varepsilon}_{H}}{\partial \rho_{i}}=\sum_{e} & \left\{(\mathbf{I}+\mathbf{R})_{e}^{t}\left(\frac{\partial \mathbf{K}_{\phi \phi}}{\partial \rho_{i}}\right)_{e}\left(\mathbf{I}+\mathbf{R}^{(m)}\right)_{e}-\left(\boldsymbol{\Phi}^{(m)}\right)_{e}^{t}\left(\frac{\partial \mathbf{K}_{\mathbf{u} \phi}}{\partial \rho_{i}}\right)_{e}\left(\mathbf{I}+\mathbf{R}^{(m)}\right)_{e}+\right. \\
& \left.+\left\{\frac{\partial \mathbf{R}^{(m)}}{\partial \rho_{i}}\right\}_{e}^{t}\left(\mathbf{K}_{\phi \phi}\right)_{e}\left(\mathbf{I}+\mathbf{R}^{(m)}\right)_{e}-\left\{\frac{\partial \boldsymbol{\Phi}^{(m)}}{\partial \rho_{i}}\right\}_{e}^{t}\left(\mathbf{K}_{\mathbf{u} \phi}\right)_{e}\left(\mathbf{I}+\mathbf{R}^{(m)}\right)_{e}\right\}
\end{aligned}
$$

Os termos $\partial \mathbf{e}(\mathbf{x}, \mathbf{y}) / \partial \rho_{i}$ e $\partial \varepsilon^{S}(\mathbf{x}, \mathbf{y}) / \partial \rho_{i}$ podem ser vistos nas Eqs. (2.54) e (2.62), respectivamente. Novamente, ao invés de se calcular explicitamente os termos $\partial\left(\nabla_{\mathbf{y}} \mathbf{R}(\mathbf{x}, \mathbf{y})\right) / \partial \rho_{i}$ e $\partial\left(\partial_{\mathbf{y}} \mathbf{\Phi}(\mathbf{x}, \mathbf{y})\right) / \partial \rho_{i}$, calcula-se a terceira e quarta parcela da Eq. (2.67) pelo método adjunto. Assim, reescrevendo a terceira parcela da Eq. (2.68), tem-se que:

$$
\left\{\frac{\partial \mathbf{R}^{(m)}}{\partial \rho_{i}}\right\}_{e}^{t}\left(\mathbf{K}_{\varphi \varphi}\right)_{e}\left(\mathbf{I}+\mathbf{R}^{(m)}\right)_{e}=\left(\mathbf{M}^{(m)}\right)^{t} \mathbf{C}_{e}
$$

Utilizando a Eq. (2.45), tem-se que:

$$
\begin{aligned}
\left(\mathbf{M}^{(m)}\right)^{t} \mathbf{K}^{-1} \mathbf{H}_{e l}\left(\mathbf{K}_{\mathbf{u} \varphi}\right)_{e}\left(\mathbf{I}+\mathbf{R}^{(m)}\right)_{e} & =\left(\mathbf{M}^{(m)}\right)^{t} \mathbf{C}_{e} \\
\mathbf{K C}_{e} & =\mathbf{W}_{e}
\end{aligned}
$$

Obtendo-se $\mathbf{C}_{e}$, pode-se calcular a Eq. (2.69).

Já a quarta parcela da Eq. (2.68) pode ser reescrita por:

$$
\left\{\frac{\partial \boldsymbol{\Phi}^{(m)}}{\partial \rho_{i}}\right\}_{e}^{t}\left(\mathbf{K}_{\mathbf{u} \varphi)_{e}}\left(\mathbf{I}+\mathbf{R}^{(m)}\right)_{e}=\left(\mathbf{M}^{(m)}\right)^{t} \mathbf{D}_{e}\right.
$$

Utilizando a Eq. (2.47), tem-se que:

$$
\begin{aligned}
\left(\mathbf{M}^{(m)}\right)^{t} \mathbf{K}^{-1} \mathbf{H}_{e l}\left(\mathbf{K}_{\mathbf{u} \varphi}\right)_{e}\left(\mathbf{I}+\mathbf{R}^{(m)}\right)_{e} & =\left(\mathbf{M}^{(m)}\right)^{t} \mathbf{D}_{e} \\
\mathbf{K D}_{e} & =\mathbf{Z}_{e}
\end{aligned}
$$

Obtendo-se $\mathbf{D}_{e}$, pode-se calcular a Eq. (2.71).

\section{- Derivada da formulação OMD}

Em relação à formulação OMD, derivando a Eq. (2.32) tem-se que: 


$$
\frac{\partial \mathbf{e}_{m a t_{j}}^{e}}{\partial \gamma_{n}^{e}}=\sum_{i=1}^{n^{e}} \frac{\partial w_{i}}{\partial \gamma_{n}^{e}} \mathbf{e}_{i} \quad \text { e } \quad \frac{\partial w_{i}}{\partial \gamma_{n}^{e}}=\frac{\frac{\partial \hat{w}_{i}}{\partial \gamma_{n}^{e}}}{\sum_{k=1}^{n_{c}} \hat{w}_{k}}
$$

onde

$$
\frac{\partial \hat{w}_{i}}{\partial \gamma_{n}^{e}}=\left\{\begin{array}{c}
p_{\gamma}\left(\gamma_{n}^{e}\right)^{p_{\gamma}-1} \prod_{j=1, j \neq i}^{n^{e}}\left(1-\left(\gamma_{j}^{e}\right)^{p_{\gamma}}\right) \\
-\left(\gamma_{i}^{e}\right)^{p_{\gamma}} p_{\gamma}\left(\gamma_{n}^{e}\right)^{p_{\gamma}-1} \prod_{j=1, j \neq i, j \neq n}^{n^{e}}\left(1-\left(\gamma_{j}^{e}\right)^{p_{\gamma}}\right) \text { se } n=i
\end{array}\right.
$$

\subsubsection{Formulação do Problema na Forma Discreta}

A definição do problema de otimização é reescrita na forma discreta da seguinte forma:

$$
\begin{array}{ll}
\underset{d_{I}, \gamma_{I}^{\alpha}}{\operatorname{Maximizar}:} & F\left(d_{I}, \gamma_{I}^{\alpha}\right)=k \\
\text { Restrito a: } & \text { Equações discretas }(2.28) \text { a }(2.29) \\
& 0 \leq d_{I} \leq 1 \quad I=1 . . N V P \\
& 0 \leq \gamma_{I}^{\alpha} \leq 1 \quad \alpha=1 . . N A C \\
& \text { condições de simetria } \\
& \text { controle de gradação e complexidade }
\end{array}
$$

onde $F$ é a função objetivo a ser maximizada, $d_{I}$ são as variáveis de projeto definidas em cada nó dos elementos finitos pertencentes ao domínio de projeto e $\gamma_{I}^{\alpha}$ representa o ângulo candidato $\alpha$ do nó $I$. O valor de $N V P$ é o total de variáveis de projeto e $N A C$ é o número de possíveis ângulos candidatos a polarização piezelétrica. O valor $d_{I}=1$ representa o material $\mathrm{A}, d_{I}=0$ representa o material B. Conforme imposto pelo método OMD, o valor de $\gamma_{I}^{\alpha}$ será igual a 1 em apenas um índice $\alpha$ no elemento $I$, de forma que as propriedades piezelétricas sejam definidas por apenas um ângulo de polarização em cada nó ao final da otimização.

A Fig. 2.22 ilustra o fluxograma que descreve o algoritmo de otimização implementado. Os elementos utilizados são isoparamétrico bilinear, com formulação de estado plano de deformação, e poligonal, com um ponto de integração por triangularização (TALISCHI et al., 2009). Primeiramente montam-se as matrizes elásticas, piezelétricas e dielétricas dos materiais base. A primeira iteração deve ser iniciada com uma distribuição inicial da distribuição de $d_{N}$ e $\gamma_{N}$. Esta distribuição inicial não pode ser uniforme, pois caso contrário os gradientes teriam valores iguais para todas as variáveis de projeto e, portanto, o método de otimização não teria uma direção preferencial para escolha. Assim, a distribuição adotada é aleatória. Definida a distribuição inicial, aplica-se a função de 
Domínio de Projeto

(1/4 da célula unitária)

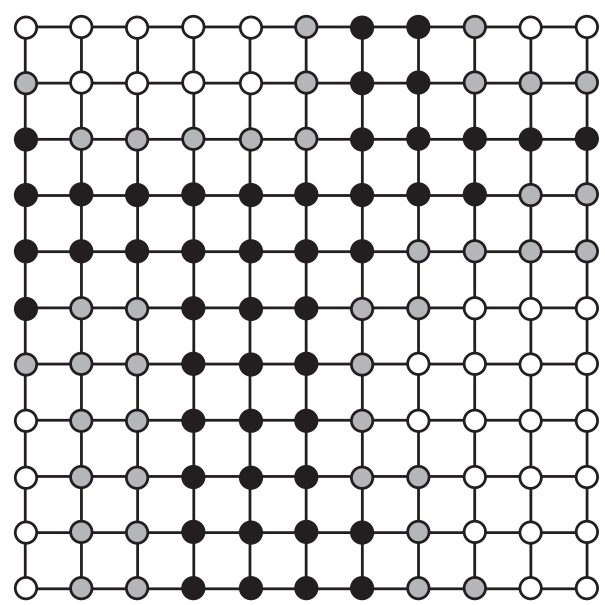

- material 1

- material 2

- material intermediário

Figura 2.21: Domínio de projeto: exemplo de malha de $10 \times 10$ elementos (as variáveis de projeto são definidas para cada nó).

projeção desejada e calculam-se as propriedades homogeneizadas. No processo iterativo, calculam-se a função objetivo e as sensibilidades, as quais são parâmetros de entrada do algoritmo de otimização. O problema de otimização é resolvido através do algoritmo MMA (Method of Moving Asymptotes) (SVANBERG, 1987). Da otimização obtém-se um novo conjunto de variáveis de projeto. Novamente são calculadas as propriedades homogeneizadas e é verificada a convergência, comparando-se os valores da função objetivo atual e anteriores. As iterações procedem até que a variação percentual da função objetivo seja menor que $10^{-3}$ (definida por experiência) ou que ocorram 50 iterações. O problema é intrinsecamente não-único. Dependendo da distribuição inicial pode-se obter diferentes topologias, ou muitas definições de periodicidades para uma mesma célula unitária.

A seguir, são mostrados os resultados obtidos da otimização topológica, visando o projeto otimizado de materiais utilizados para coleta de energia.

\subsection{Resultados}

Nesta seção são apresentados exemplos numéricos de microestruturas otimizadas. Primeiramente, são obtidas células unitárias sem gradação de material, a fim de se verificar as influências da malha de elementos finitos e da direção de polarização. Posteriormente, a verificação das influências de ambos os parâmetros é realizada novamente para células unitárias com gradação de material. O domínio de projeto adotado é de um quarto de uma célula unitária quadrada bidimensional, a fim de se reduzir o custo computacional. Assim, antes de se iniciar o processo iterativo de otimização, é necessário mapear os valores nodais em toda a célula unitária, em função das variáveis de projeto do domínio de projeto. Em todas as simulações apresentadas nesta seção a distribuição de material é 


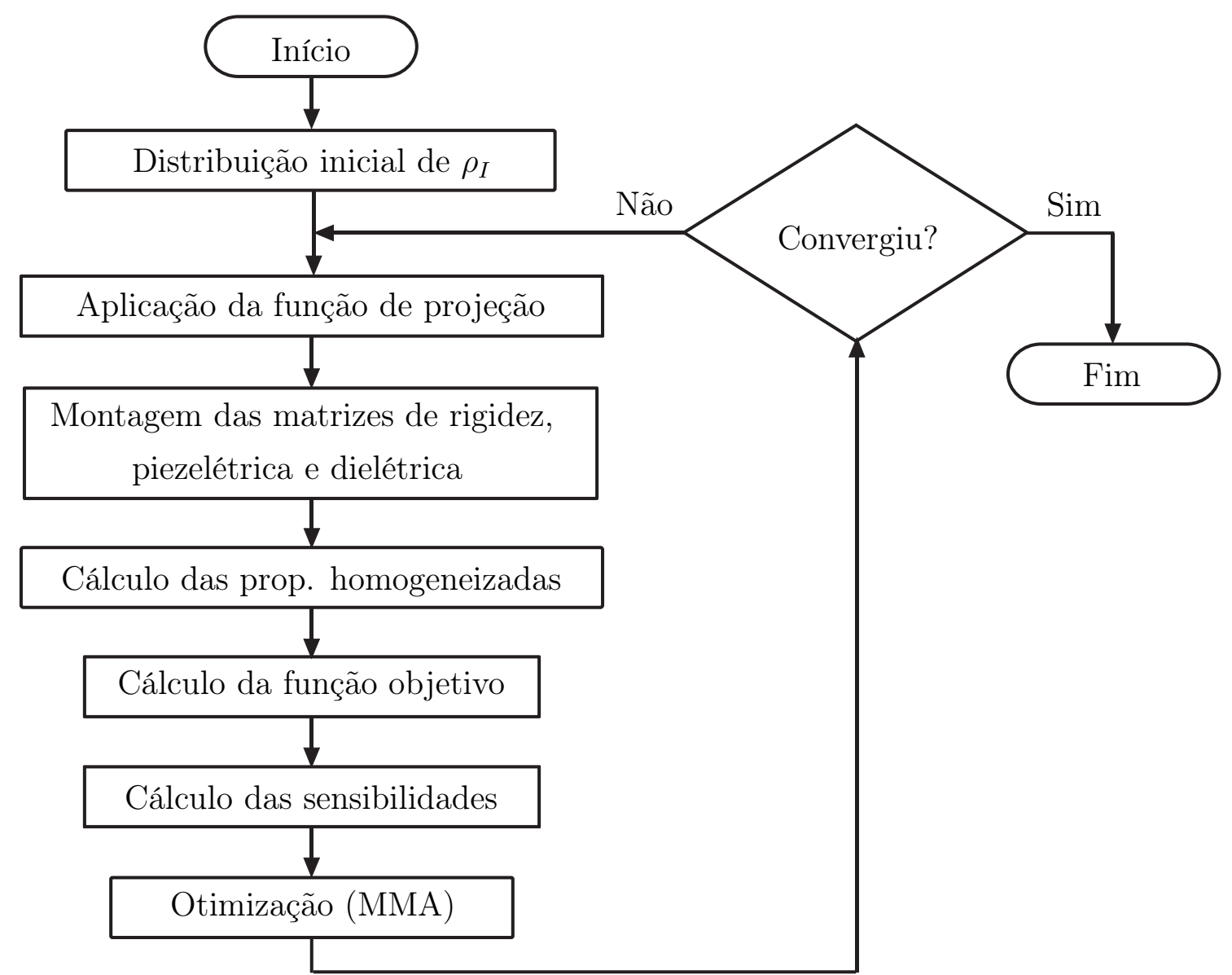

Figura 2.22: Fluxograma do algoritmo de otimização.

simétrica em relação aos eixos $x$ e $y$. Para os casos onde a direção de polarização é livre, a direção de polarização é simétrica apenas em relação ao eixo $y$; em relação ao eixo $x$, a direção de polarização dos valores nodais é idêntica a das respectivas variáveis de projeto. A Fig. 2.23 ilustra este esquema de mapeamento dos valores nodais. Todas as simulações são realizadas para a célula unitária inteira, aplicando-se condições de periodicidade. O domínio projeto é discretizado em 81 elementos, de modo que a malha quadrilátera seja formada por $9 \times 9$ elementos e na malha poligonal os elementos são gerados de acordo com algoritmo de Lloyd apresentado na Seção 2.3.2. Os materiais adotados são PZT-5A e epóxi, cujas propriedades estão listadas na Tabela 2.4. Os coeficientes de penalização adotados tem valores iguais a $p_{c}=2, p_{e}=2$ e $p_{\varepsilon}=4$, de acordo com as duas condições para que haja uma convergência estável do problema de otimização (KIM et al., 2010).

No caso com variação de direção de polarização do PZT-5A, adotam-se três ângulos distintos: $0^{\circ}, 45^{\circ}$ e $90^{\circ}$. Os resultados apresentados neste capítulo são obtidos utilizando-se $p_{\gamma}=6$. Cerca de 10 simulações são realizadas para cada caso e o melhor desempenho obtido é apresentado na próxima seção. O coeficiente de acoplamento eletromecânico $k$ do material PZT-5A puro é igual a 0,145. Variando-se a fração de volume do piezocompósito 2-2 (ver Fig. 2.24b), o máximo valor de $k$ obtido é igual ao do PZT-5A puro (SILVA et al., 1999). Ambos os casos são para a polarização fixada na vertical. Esses resultados servirão 


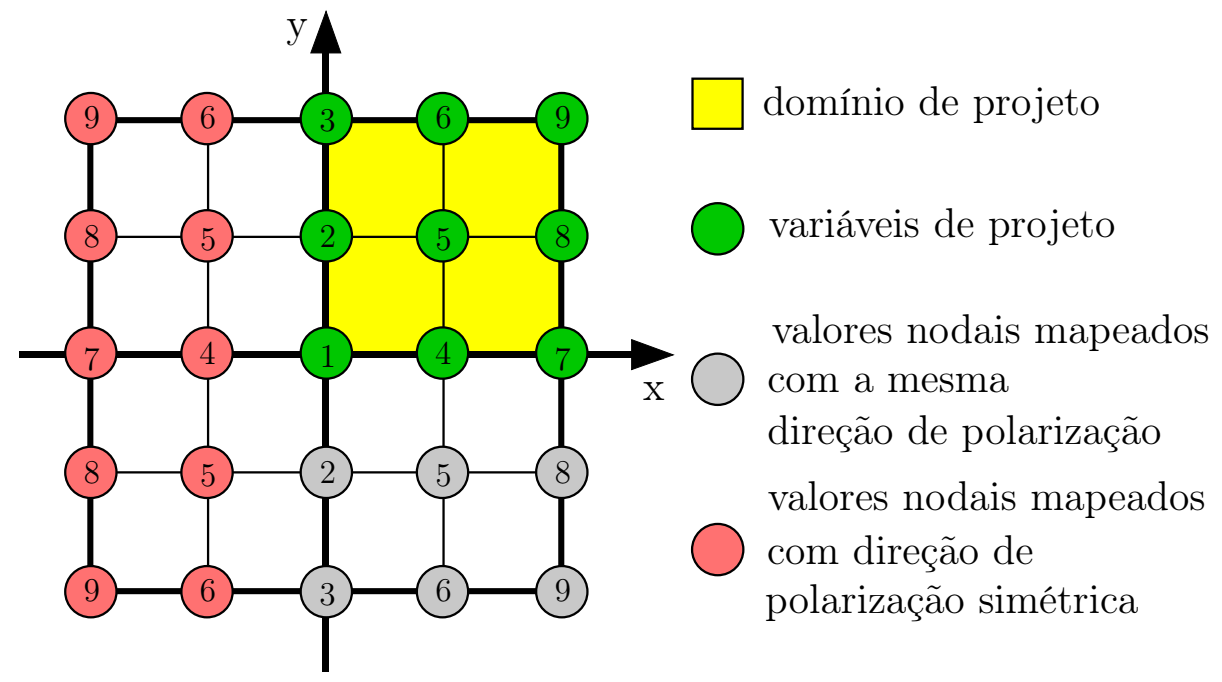

Figura 2.23: Desenho esquemático do domínio de projeto em $1 / 4$ da célula unitária formada por $2 \times 2$ elementos e o mapeamento dos valores nodais das pseudo-densidades de material e das direções de polarização.

de referência para os resultados obtidos do processo de otimização.

Tabela 2.4: Propriedades dos materiais PZT-5A e polímero epóxi.

\begin{tabular}{|c|c|c|}
\hline Propriedade & PZT-5A & Epóxi \\
\hline \hline$c_{11}^{E}\left(10^{10} \mathrm{~N} / \mathrm{m}^{2}\right)$ & 12,1 & 0,53 \\
\hline$c_{12}^{E}\left(10^{10} \mathrm{~N} / \mathrm{m}^{2}\right)$ & 7,54 & 0,31 \\
\hline$c_{13}^{E}\left(10^{10} \mathrm{~N} / \mathrm{m}^{2}\right)$ & 7,52 & 0,31 \\
\hline$c_{33}^{E}\left(10^{10} \mathrm{~N} / \mathrm{m}^{2}\right)$ & 11,1 & 0,53 \\
\hline$c_{13}^{E}\left(10^{10} \mathrm{~N} / \mathrm{m}^{2}\right)$ & 2,10 & 0,11 \\
\hline$e_{13}\left(C / \mathrm{m}^{2}\right)$ & $-5,4$ & 0 \\
\hline$e_{33}\left(C / \mathrm{m}^{2}\right)$ & 15,8 & 0 \\
\hline$e_{15}\left(C / m^{2}\right)$ & 12,3 & 0 \\
\hline$\varepsilon_{11}^{S} / \varepsilon_{0}{ }^{1}$ & 1650 & 4 \\
\hline$\varepsilon_{33}^{S} / \varepsilon_{0}{ }^{1}$ & 1700 & 4 \\
\hline Peso específico $\left(\mathrm{kg} / \mathrm{m}^{3}\right)$ & 7500 & 1384 \\
\hline \multicolumn{2}{|c|}{${ }^{1} \varepsilon_{0}=8,854188 \cdot 10^{-12} \mathrm{~F} / \mathrm{m}$} \\
\hline
\end{tabular}

Para avaliar as tensões mecânicas no interior da célula unitária, é preciso definir um modelo macroscópico, com condições de contorno e carregamento bem definidos. O modelo adotado consiste num bloco retangular com $20 \mathrm{~mm}$ de comprimento e $5 \mathrm{~mm}$ de espessura, de material homogêneo, e com um carregamento distribuído $\sigma_{0}$, conforme mostrado na Fig. 2.24a. As propriedades do material homogêneo consideradas são as propriedades efetivas obtidas para cada solução otimizada. Este modelo macroscópico é simulado no estado plano de deformações.

A partir da análise macroscópica, obtém-se as deformações e potenciais elétricos macroscópicos, os quais são uniformes em todo o modelo. Em seguida, as tensões mecânicas microscópicas são calculadas para cada célula unitária utilizando a Eq. (2.15). As distribuições das microtensões mecânicas são mostradas juntamente com as células 


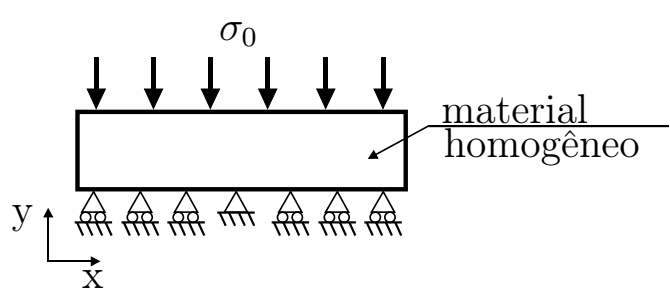

(a)

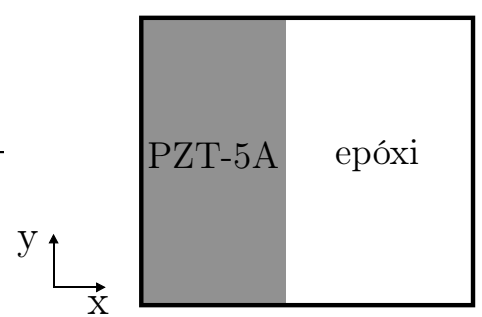

(b)

Figura 2.24: (a) Modelo adotado para se calcular as tensões mecânicas macroscópicas e (b) exemplo de célula unitária do piezocompósito 2-2.

unitárias nas próximas figuras.

\subsubsection{Projeto de materiais sem gradação de material}

A primeira abordagem é verificar a influência do tipo de elemento finito utilizado e da direção de polarização na função objetivo, sem considerar gradação de material na célula unitária. A Fig. 2.25 mostra a célula unitária otimizada utilizando-se uma malha formada por elementos finitos quadriláteros e direção de polarização fixada na vertical, a matriz periódica formada por $3 \times 3$ células unitárias e a distribuição de microtensões mecânicas no interior da célula unitária. A Fig. 2.26 mostra o resultado utilizando-se uma malha de elementos finitos poligonais e direção de polarização fixada na vertical. Comparando ambas as figuras, nota-se que utilizando-se a malha poligonal é possível obter uma topologia com arredondamento mais suave do que a obtida com a malha quadrilátera. Além disso, nota-se também que a máxima tensão é menor no caso da malha poligonal. As Fig. 2.27 e 2.28 mostram os resultados otimizados para uma malha de elementos quadriláteros e poligonais, respectivamente, com direção de polarização livre através do conceito de OMD. Comparando ambos os casos, nota-se novamente que a topologia obtida com a malha quadrilátera segue a própria estrutura da malha, isto é, a distribuição de material apresenta contornos mais retilíneos; já com a malha poligonal esse efeito não ocorre. As propriedades efetivas de cada solução estão listadas na tabela 2.5.

Na Tabela 2.6 pode-se observar os valores de $\mathrm{k}$ para os quatro casos otimizados, o ganho em relação ao material PZT-5A puro e piezocompósito 2-2 (cujos valores de k são iguais a 0,145) além dos valores máximos relativos das tensões mecânicas na célula unitária. Comparando-se as microestruturas otimizadas com direção de polarização fixa, nota-se que o modelo com malha poligonal (ganho $=105,5 \%$ ) apresenta um desempenho superior ao do modelo com malha quadrilátera $($ ganho $=100,7 \%$ ). Este fenômeno repete-se para os casos com direção de polarização livre, onde o modelo com malha quadrilátera possui um ganho de 113,1\% e o com malha poligonal, 120,0\%. Além disso, comparando-se a influência da direção de polarização, nota-se que os desempenhos das 


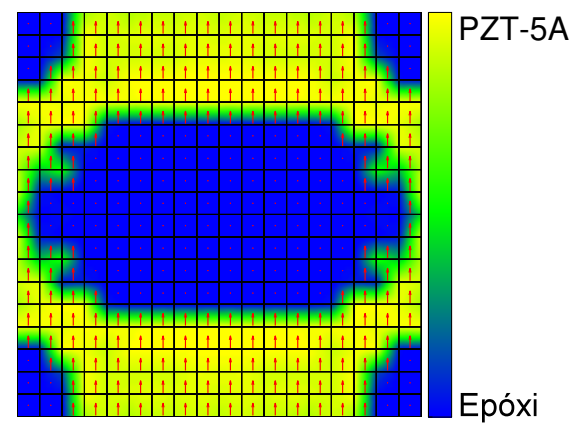

(a)

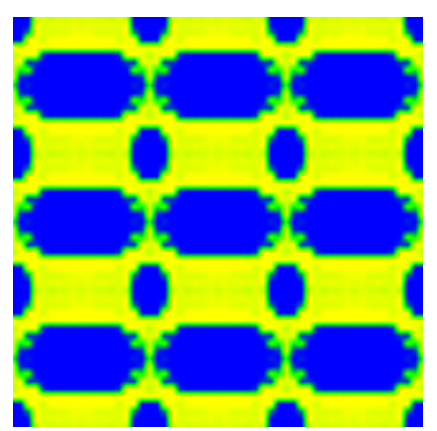

(b)

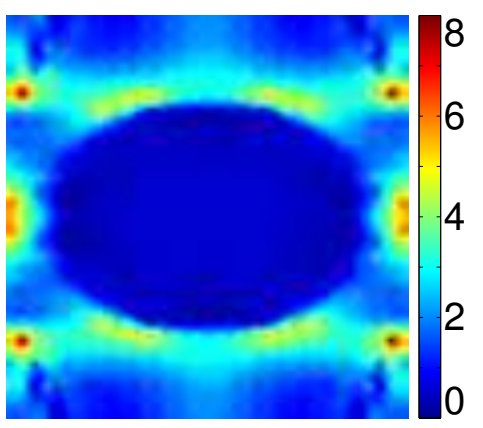

(c)

Figura 2.25: Microestrutura otimizada sem gradação de material, utilizando elementos quadriláteros e direção de polarização fixada na vertical: (a) distribuição de material, (b) matriz periódica e (c) distribuição das tensões microscópicas no interior da célula unitária.

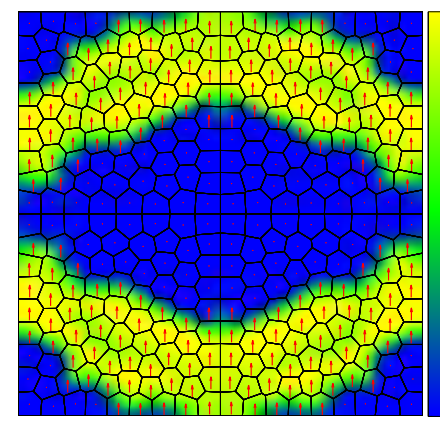

(a)

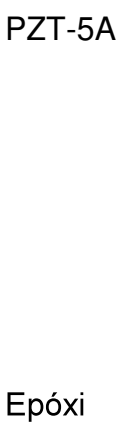

Epóxi

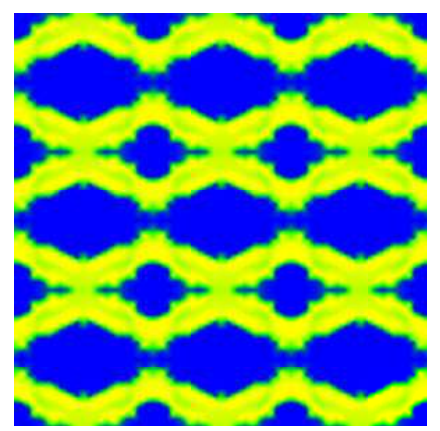

(b)

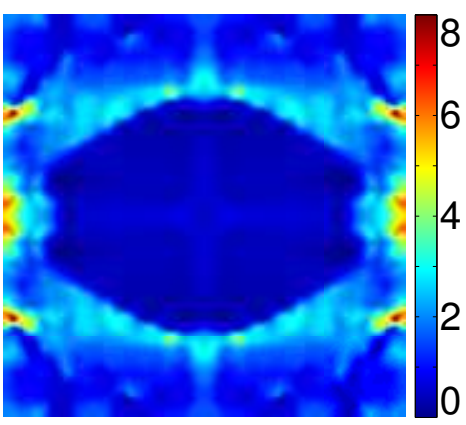

(c)

Figura 2.26: Microestrutura otimizada sem gradação de material, utilizando elementos poligonais e direção de polarização fixada na vertical: (a) distribuição de material, (b) matriz periódica e (c) distribuição das tensões microscópicas no interior da célula unitária.

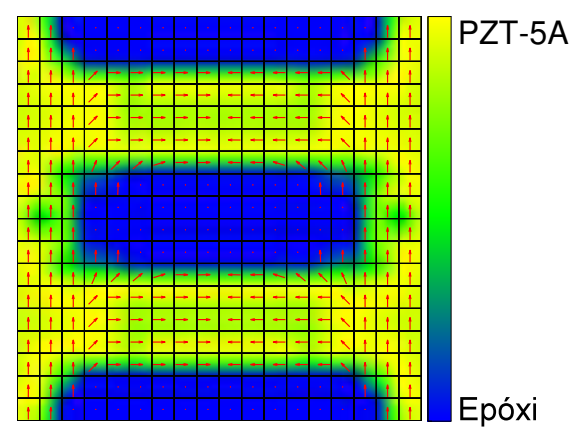

(a)

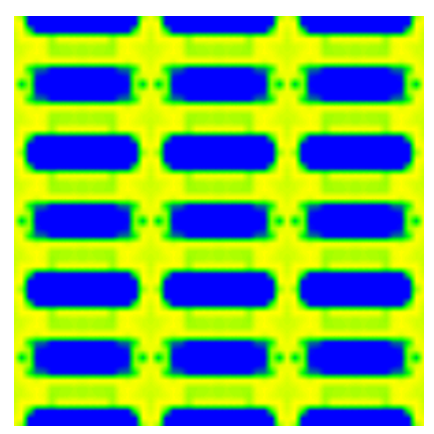

(b)

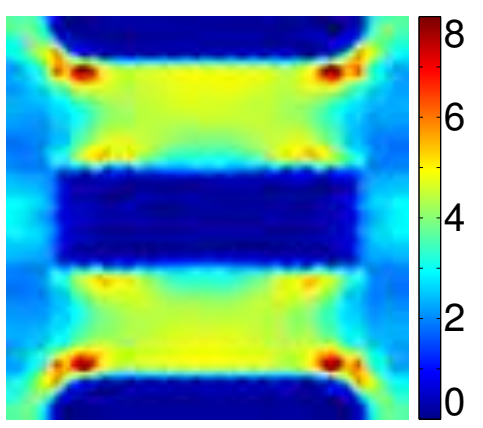

(c)

Figura 2.27: Microestrutura otimizada sem gradação de material, utilizando elementos quadriláteros e direção de polarização livre: (a) distribuição de material, (b) matriz periódica e (c) distribuição das tensões microscópicas no interior da célula unitária.

microestruturas das Fig. 2.27 e 2.28 são melhores do que os resultados obtidos com direção de polarização fixa. A distribuição de tensões mecânicas apresentadas nessas figuras correspondem às máximas tensões principais, as quais são sempre positivas. Para o caso da Fig. 2.25, nota-se que as microtensões podem alcançar valores até 7,8 vezes maiores do que a tensão obtida através do modelo macroscópico. Para os casos das Fig. 


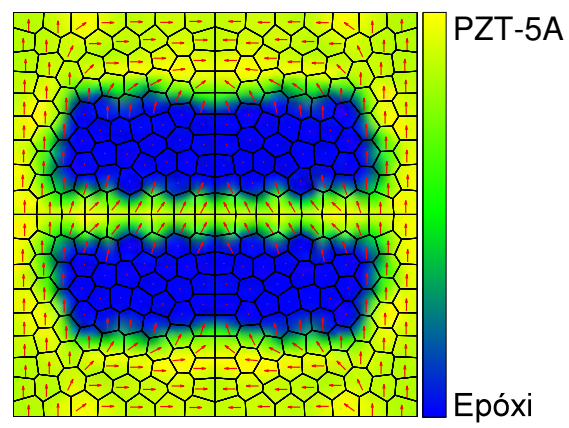

(a)

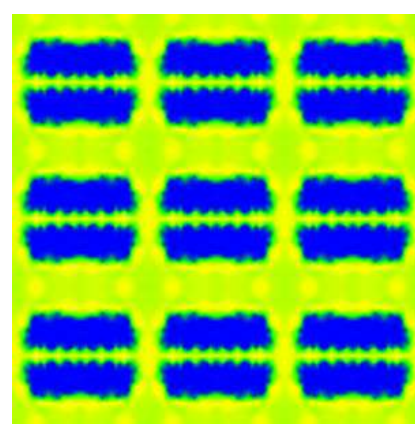

(b)

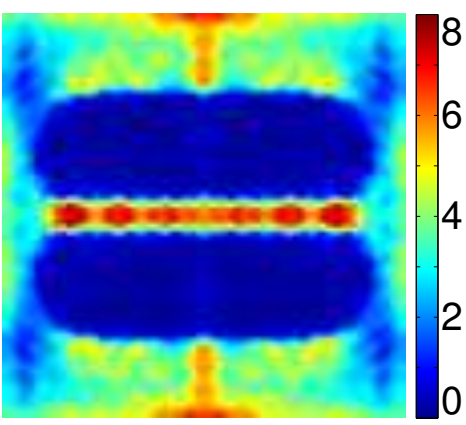

(c)

Figura 2.28: Microestrutura otimizada sem gradação de material, utilizando elementos poligonais e direção de polarização livre: (a) distribuição de material, (b) matriz periódica e (c) distribuição das tensões microscópicas no interior da célula unitária.

Tabela 2.5: Propriedades efetivas das microestruturas otimizadas sem gradação de material.

\begin{tabular}{|c|c|c|c|c|c|c|c|c|c|}
\hline Fig. & Polarização & Elemento & \multicolumn{3}{|c|}{$\mathbf{c}_{H}^{E}(\mathrm{MPa})$} & \multicolumn{2}{|c|}{$\mathbf{e}_{H}\left(C / m^{2}\right)$} & \multicolumn{2}{|c|}{$\varepsilon_{H}^{E} / \varepsilon_{0}$} \\
\hline 2.25 & vertical & quadrilátero & {$\left[\begin{array}{c}38.36 \\
16.70 \\
0\end{array}\right.$} & $\begin{array}{c}16.70 \\
27.11 \\
0\end{array}$ & $\begin{array}{c}0 \\
0 \\
6.63\end{array}$ & {$\left[\begin{array}{r}0 \\
0 \\
2.5\end{array}\right.$} & $\begin{array}{c}0.95 \\
4.70 \\
0\end{array}$ & {$\left[\begin{array}{c}906 \\
0\end{array}\right.$} & $\begin{array}{c}0 \\
548\end{array}$ \\
\hline 2.26 & vertical & poligonal & {$\left[\begin{array}{c}31.37 \\
15.30 \\
0\end{array}\right.$} & $\begin{array}{c}15.30 \\
22.96 \\
0\end{array}$ & $\begin{array}{c}0 \\
0 \\
6.53\end{array}$ & $\begin{array}{r}0 \\
0 \\
2.3\end{array}$ & $\begin{array}{c}1.49 \\
3.77 \\
0\end{array}$ & $\begin{array}{c}764 \\
0\end{array}$ & $\begin{array}{c}0 \\
498\end{array}$ \\
\hline 2.27 & livre & quad. & {$\left[\begin{array}{c}49.55 \\
13.05 \\
0\end{array}\right.$} & $\begin{array}{c}13.05 \\
24.37 \\
0\end{array}$ & $\begin{array}{c}0 \\
0 \\
4.28\end{array}$ & $\begin{array}{c}0 \\
0 \\
0.45\end{array}$ & $\begin{array}{c}-0.05 \\
3.96 \\
0\end{array}$ & {$\left[\begin{array}{c}1038 \\
0\end{array}\right.$} & $\begin{array}{c}0 \\
403\end{array}$ \\
\hline 2.28 & livre & poligonal & {$\left[\begin{array}{c}59.43 \\
17.08 \\
0\end{array}\right.$} & $\begin{array}{c}17.08 \\
29.50 \\
0\end{array}$ & $\begin{array}{c}0 \\
0 \\
5.66\end{array}$ & $\begin{array}{c}0 \\
0 \\
0.66\end{array}$ & $\begin{array}{c}-0.31 \\
4.99 \\
0\end{array}$ & {$\left[\begin{array}{c}1249 \\
0\end{array}\right.$} & $\begin{array}{c}0 \\
508\end{array}$ \\
\hline
\end{tabular}

2.26 a 2.28, os máximos valores de tensão relativos são 7,6, 7,9 e 7,5, respectivamente. A partir destes resultados, pode-se concluir que, para a mesma quantidade de elementos utilizada em todos os casos, a malha poligonal conduz o problema para melhores mínimos locais. Além disso, direções de polarização livres no interior da célula unitária oferecem um significativo aumento no desempenho das microestruturas otimizadas.

Tabela 2.6: Comparação da microestruturas otimizadas, sem gradação de material.

\begin{tabular}{|c|c|c|c|c|c|}
\hline Fig. & Polarização & Elemento & $\mathrm{k}$ & Ganho $^{1}$ & $\sigma / \sigma_{0}$ \\
\hline 2.25 & vertical & quadrilátero & 0,291 & $100,7 \%$ & 7,8 \\
\hline 2.26 & vertical & poligonal & 0,298 & $105,5 \%$ & 7,6 \\
\hline 2.27 & livre & quadrilátero & 0,309 & $113,1 \%$ & 7,9 \\
\hline 2.28 & livre & poligonal & 0,319 & $120,0 \%$ & 7,5 \\
\hline
\end{tabular}




\subsubsection{Projeto de materiais com gradação funcional}

É sabido que para atingir melhores desempenhos, os materiais compósitos devem apresentar distribuições de materiais discretas (0-1), isto é, com variações bruscas entre fases (SIGMUND, 2000a). Entretanto, é muito comum obter gradações mínimas entre os materiais nos processos de fabricação de compósitos. Portanto, uma pergunta pertinente é como essa gradação influencia no comportamento de materiais compósitos. Para responder a esta pergunta, investiga-se novamente os casos analisados na seção anterior, considerando um raio de gradação de 10\% do comprimento da célula unitária. As Fig. 2.29 a 2.32 apresentam as microestruturas otimizadas. Comparando estes resultados com os obtidos anteriormente, nota-se que o efeito de contornos mais retilíneos obtidos na malha quadrilátera não ocorre com a presença da gradação funcional. Isso mostra que o filtro de projeção utilizado diminui a dependência de malha. Entretanto, os resultados obtidos com a malha quadrilátera e poligonal diferem-se significativamente. As propriedades efetivas de cada solução estão listadas na tabela 2.7 .

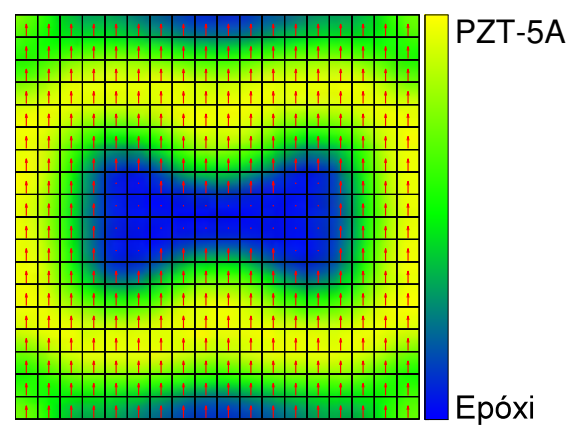

(a)

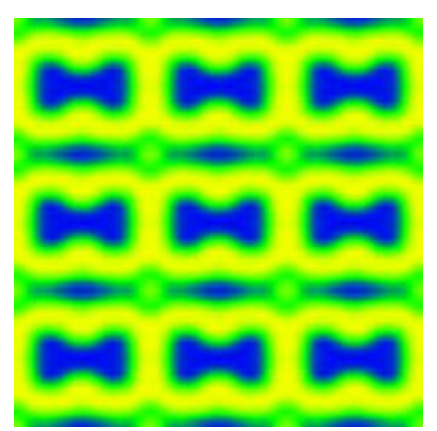

(b)

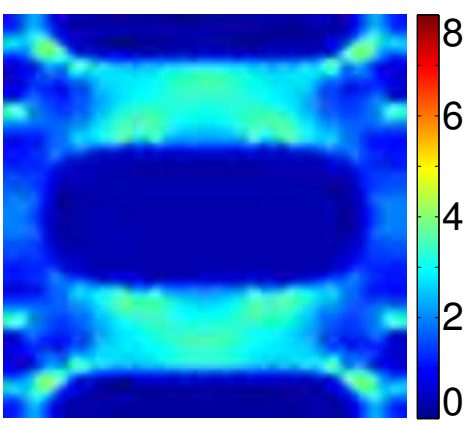

(c)

Figura 2.29: Microestrutura otimizada com gradação de material, utilizando elementos quadriláteros e direção de polarização fixada na vertical: (a) distribuição de material, (b) matriz periódica e (c) distribuição das tensões microscópicas no interior da célula unitária.

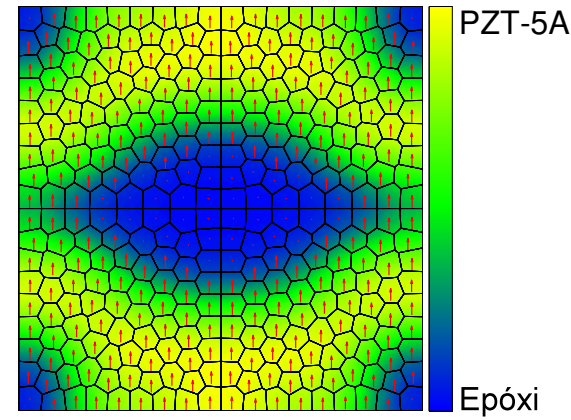

(a)

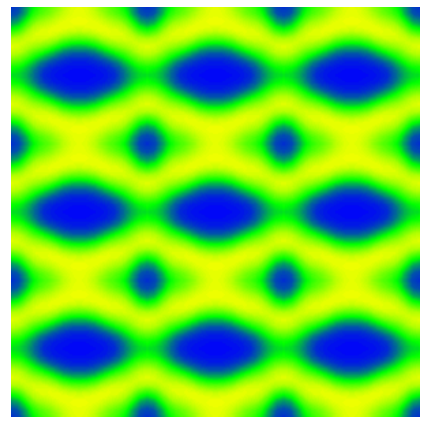

(b)

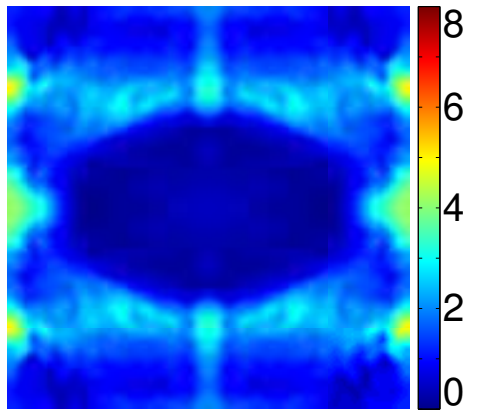

(c)

Figura 2.30: Microestrutura otimizada com gradação de material, utilizando elementos poligonais e direção de polarização fixada na vertical: (a) distribuição de material, (b) matriz periódica e (c) distribuição das tensões microscópicas no interior da célula unitária. 


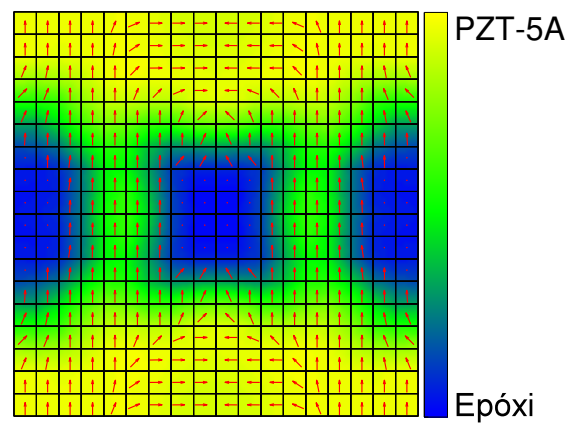

(a)

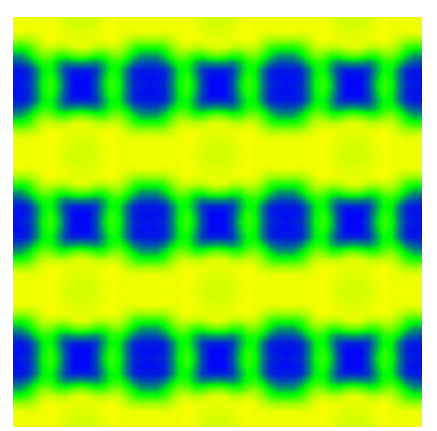

(b)

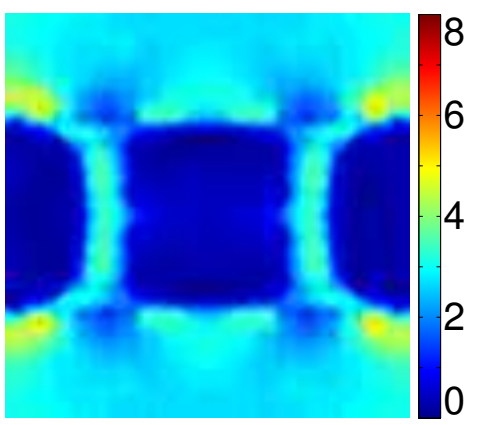

(c)

Figura 2.31: Microestrutura otimizada com gradação de material, utilizando elementos quadriláteros e direção de polarização livre: (a) distribuição de material, (b) matriz periódica e (c) distribuição das tensões microscópicas no interior da célula unitária.

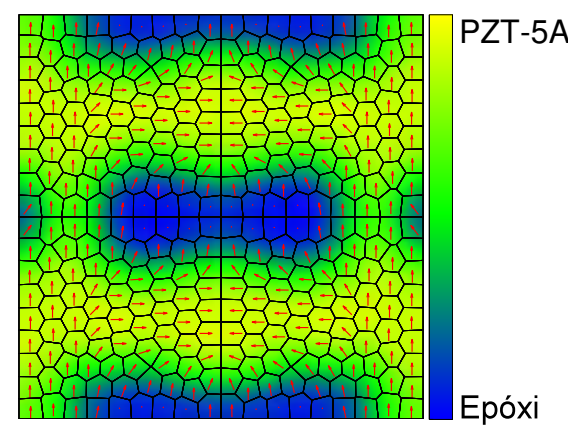

(a)

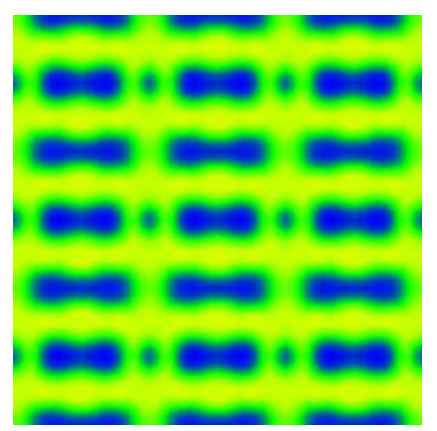

(b)

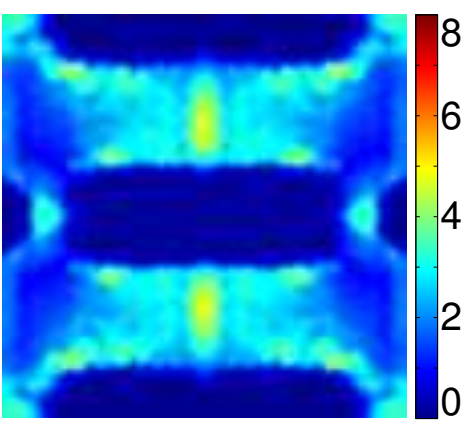

(c)

Figura 2.32: Microestrutura otimizada com gradação de material, utilizando elementos poligonais e direção de polarização livre: (a) distribuição de material, (b) matriz periódica e (c) distribuição das tensões microscópicas no interior da célula unitária.

A convergência da função objetivo ao longo das iterações pode ser vista na Fig. 2.33, que mostra o gráfico da função objetivo para o modelo com gradação funcional, polarização livre e malha poligonal (Fig. 2.32).

Na Tabela 2.8 pode-se observar os valores do coeficiente $k$ para os quatro casos otimizados das microestruturas com gradação funcional e o ganho em relação ao material PZT-5A puro. Comparando-se as microestruturas otimizadas tanto com direção de polarização fixa ou livre, nota-se que os modelos com malha poligonal apresentam desempenho superior aos dos modelos com malha quadrilátera, similar aos resultados apresentados anteriormente para os casos sem gradação de material. Comparando-se os valores máximos relativos das tensões mecânicas, novamente, os modelos com elementos poligonais apresentam menores valores do que os obtidos com elementos quadriláteros. Comparando-se a influência da gradação de material, nota-se que os ganhos do coeficiente $k$ são menores do que os obtidos sem gradação de material. Entretanto, as microestruturas com gradação de material mostradas nas Fig. 2.29 a 2.32 apresentam menores valores de microtensões relativas quando comparados aos casos sem gradação de material das Fig. 2.25 a 2.28. Portanto, embora os valores das funções objetivos sejam menores com a 
Tabela 2.7: Propriedades efetivas das microestruturas otimizadas com gradação de material.

\begin{tabular}{|c|c|c|c|c|c|c|c|c|c|}
\hline Fig. & Polarização & Elemento & \multicolumn{3}{|c|}{$\mathbf{c}_{H}^{E}(\mathrm{MPa})$} & \multicolumn{2}{|c|}{$\mathbf{e}_{H}\left(C / m^{2}\right)$} & \multicolumn{2}{|c|}{$\varepsilon_{H}^{E} / \varepsilon_{0}$} \\
\hline 2.29 & vertical & quadrilátero & {$\left[\begin{array}{c}42.68 \\
13.32 \\
0\end{array}\right.$} & $\begin{array}{c}13.32 \\
22.92 \\
0\end{array}$ & $\begin{array}{c}0 \\
0 \\
4.63\end{array}$ & {$\left[\begin{array}{r}0 \\
0 \\
1.7\end{array}\right.$} & $\begin{array}{c}0.04 \\
3.60 \\
0\end{array}$ & {$\left[\begin{array}{c}984 \\
0\end{array}\right.$} & $\left.\begin{array}{c}0 \\
402\end{array}\right]$ \\
\hline 2.30 & vertical & poligonal & {$\left[\begin{array}{c}28.25 \\
13.48 \\
0\end{array}\right.$} & $\begin{array}{c}13.48 \\
20.93 \\
0\end{array}$ & $\begin{array}{c}0 \\
0 \\
5.85\end{array}$ & {$\left[\begin{array}{r}0 \\
0 \\
2.1\end{array}\right.$} & $\begin{array}{c}1.24 \\
3.05 \\
0\end{array}$ & $\begin{array}{c}712 \\
0\end{array}$ & $\left.\begin{array}{c}0 \\
378\end{array}\right]$ \\
\hline 2.31 & livre & quadrilátero & {$\left[\begin{array}{c}57.32 \\
17.55 \\
0\end{array}\right.$} & $\begin{array}{c}17.55 \\
32.09 \\
0\end{array}$ & $\begin{array}{c}0 \\
0 \\
5.36\end{array}$ & {$\left[\begin{array}{c}0 \\
0 \\
1.32\end{array}\right.$} & $\begin{array}{c}-0.12 \\
5.40 \\
0\end{array}$ & {$\left[\begin{array}{c}1209 \\
0\end{array}\right.$} & $\begin{array}{c}0 \\
565\end{array}$ \\
\hline 2.32 & livre & poligonal & {$\left[\begin{array}{c}57.66 \\
16.52 \\
0\end{array}\right.$} & $\begin{array}{c}16.52 \\
28.76 \\
0\end{array}$ & $\begin{array}{c}0 \\
0 \\
5.86\end{array}$ & $\begin{array}{c}0 \\
0 \\
0.86\end{array}$ & $\begin{array}{c}-0.28 \\
4.63 \\
0\end{array}$ & {$\left[\begin{array}{c}1213 \\
0\end{array}\right.$} & $\begin{array}{c}0 \\
474\end{array}$ \\
\hline
\end{tabular}

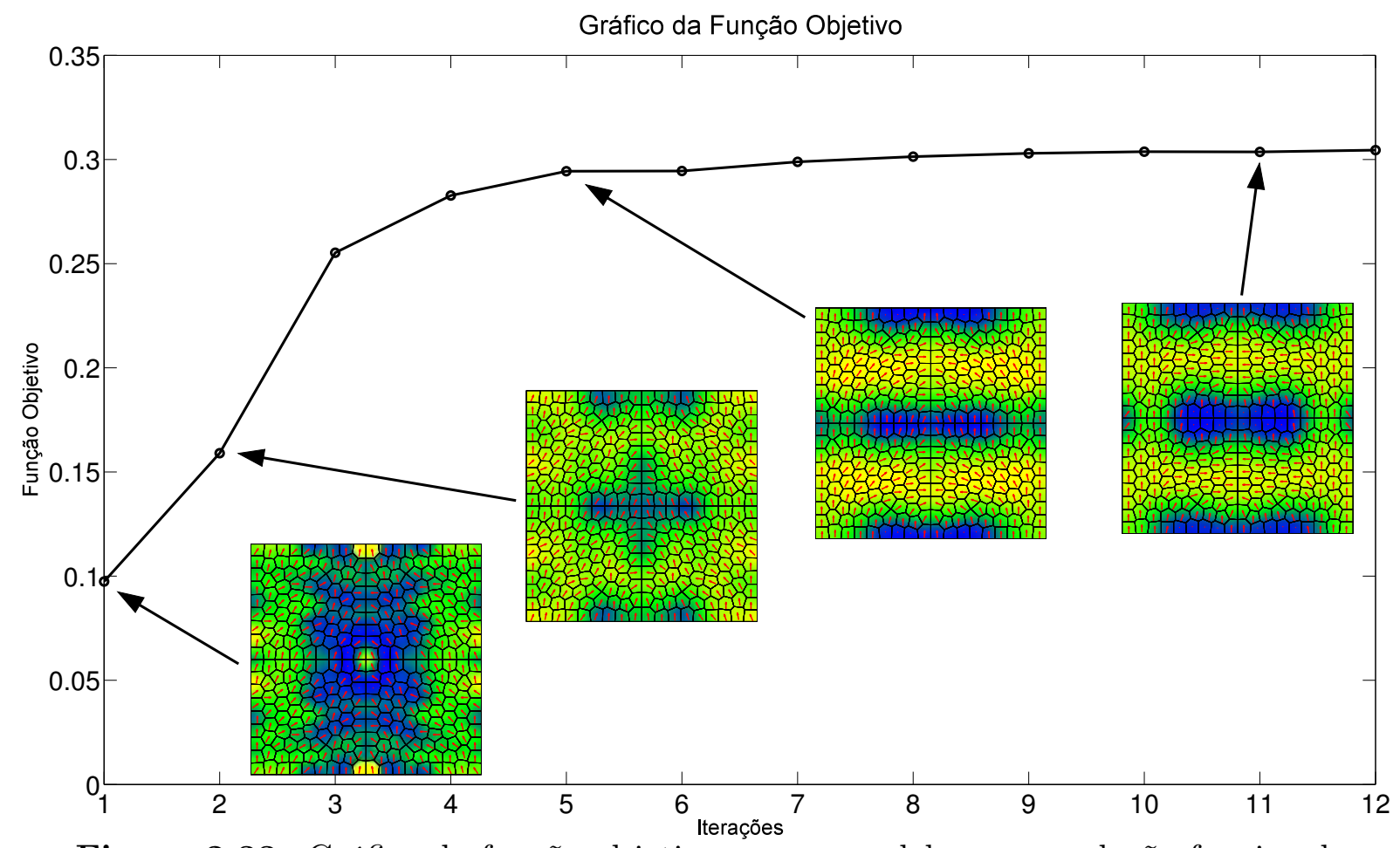

Figura 2.33: Gráfico da função objetivo para o modelo com gradação funcional, polarização livre e malha poligonal (Fig. 2.32). 
presença da gradação de material, os valores máximos das microtensões relativas também diminuem. Assim, existe uma relação de compromisso entre a maximização do coeficiente $k$ e os valores máximos relativos das microtensões na célula unitária.

Tabela 2.8: Comparação da microestruturas otimizadas, com gradação de material.

\begin{tabular}{|c|c|c|c|c|c|}
\hline Fig. & Polarização & Elemento & $\mathrm{k}$ & Ganho $^{1}$ & $\sigma / \sigma_{0}$ \\
\hline 2.29 & vertical & quadrilátero & 0,285 & $96,6 \%$ & 5,7 \\
\hline 2.30 & vertical & poligonal & 0,294 & $102,8 \%$ & 5,1 \\
\hline 2.31 & livre & quadrilátero & 0,299 & $106,2 \%$ & 5,9 \\
\hline 2.32 & livre & poligonal & 0,305 & $110,3 \%$ & 6,0 \\
\hline
\end{tabular}

\subsection{Considerações Finais}

Este capítulo apresenta uma metodologia para projeto de MPGF, que considera importantes aspectos no processo de projeto de materiais piezocompósitos visando aplicações de coleta de energia, tais como: influência de diferentes direções de polarização piezelétrica, influência da gradação de materiais entre as fases constituintes na célula unitária e influência dos elementos finitos quadriláteros e poligonais. A variação da direção de polarização é implementada utilizando o conceito OMD.

A partir dos exemplos de microestruturas obtidas neste capítulo, conclui-se que as direções de polarização variáveis na célula unitária são fatores importantes no projeto de MPGF e permitem obter microestruturas com melhores desempenhos. Além disso, utilizando o mesmo número de elementos finitos, malhas poligonais não estruturadas conduzem o problema a melhores mínimos locais, quando comparadas a malhas quadriláteras estruturadas.

A capacidade do método de homogeneização de determinar microtensões mostra que, para piezocompósitos, valores de tensão efetivos calculados através de um modelo em escala macroscópica podem induzir a uma má seleção de materiais em projetos estruturais. Tais valores de tensão macroscópicos podem ser menores que valores máximos de tensão ocasionados na microestrutura, os quais podem gerar microdefeitos no material. Assim, comprova-se novamente que o método de homogeneização apresenta-se como uma poderosa ferramenta no projeto de materiais com propriedades específicas para cada aplicação, pois é possível obter informações detalhadas do campo de tensões microscópicos que ocorrem na microestrutura.

É sabido que a aplicação do conceito de MGF no projeto de materiais utilizando o MOT reduz a concentração de tensões mecânicas entre os materiais constituintes, pois a eliminação de interfaces naturalmente evita este problema. Portanto, torna-se 
desnecessária a consideração da concentração de tensões mecânicas na função objetivo ou nas restrições do problema de otimização. Entretanto, neste trabalho o foco é quantificar numericamente a redução destas tensões no projeto de materiais piezocompósitos. Dos exemplos apresentados neste trabalho, verifica-se que as concentrações de microtensões podem ser redistribuídas na célula unitária em função da gradação dos materiais. Assim, embora estruturas discretas apresentem valores maiores de $k$, estas também podem apresentar maiores valores de microtensões na célula unitária. Desse modo, para evitar falhas, estas estruturas suportarão menores carregamentos externos e, consequentemente, converterão menos energia mecânica em elétrica. Por outro lado, embora estruturas com gradação funcional apresentem valores menores de $k$, estas apresentam também menores valores de microtensões na célula unitária. Assim, podem suportar maiores carregamentos externos e, consequentemente, podem converter mais energia. Portanto, há uma solução de compromisso entre a maximização do coeficiente de acoplamento eletromecânico e das concentrações de tensões microscópicas no interior da célula unitária. 


\section{Projeto de Materiais Piezocompósitos com Gradação Funcional Visando Aplicações Fonônicas}

O método da homogeneização implementado no capítulo anterior pode ser utilizado apenas para aplicações estáticas, isto é, quando o comprimento de onda do fenômeno de interesse é muito maior do que o tamanho da célula unitária. Porém, o interesse neste trabalho é projetar também materiais piezocompósitos para aplicações dinâmicas. Uma propriedade relevante dos materiais compósitos é a possibilidade de ter faixas de frequência, mais conhecidas por band gaps, nas quais ondas elásticas não se propagam (VASSEUR et al., 1998; TANAKA; TOMOYASU; TAMURA, 2000; SIGMUND; JENSEN, 2003). A Fig. 3.1 ilustra um exemplo deste efeito. No material sem band gap, uma onda elástica propaga-se livremente, da esquerda para a direita; já no material com band gap, a onda é atenuada, conforme ela atravessa o material.

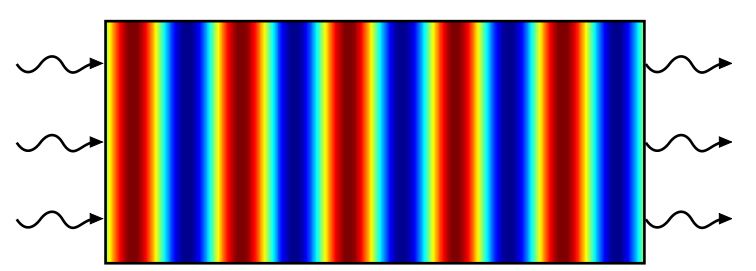

(a)

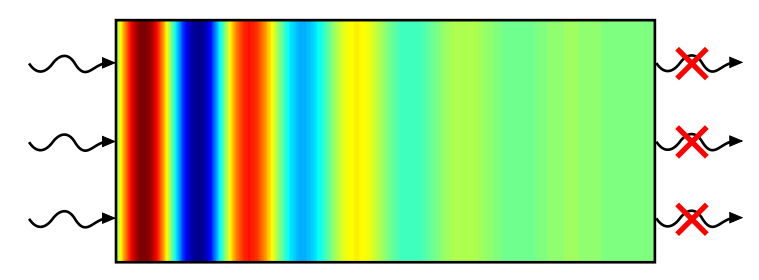

(b)

Figura 3.1: Propagação de uma onda elástica em um material (a) sem band gap e (b) com band gap.

A propagação de ondas elásticas num meio heterogêneo tem comportamento dispersivo, isto é, a onda se decompõe em múltiplas ondas de diferentes frequências. Esse fenômeno de atenuação ocorre devido ao princípio de interferências destrutivas das ondas que sofrem espalhamento (HUSSEIN et al., 2006). Materiais que apresentam band gaps acústicos, denominados por materiais fonônicos, são de grande interesse para diversas aplicações, tais como transdutores, filtros acústicos, controle de ruído, vibração, entre outros (GONELLA; TO; LIU, 2009). Portanto, é essencial dominar o projeto de materiais compósitos com band gaps maximizados ou, de preferência, com posição e 
largura prescritas. Diversos trabalhos tem sido realizados focando o aumento da largura dos band gaps (SIGMUND; JENSEN, 2003; ZHANG et al., 2003; WU et al., 2009; WEI et al., 2009) e uma das conclusões é que a largura dos band gaps são determinadas pelo contraste das propriedades elásticas dos materiais constituintes, assim como pela fração de volume destes na célula unitária da matriz periódica.

O fenômeno gerador dos band gaps é a reflexão de ondas entre as fases constituintes do material compósito. A reflexão das ondas é maximizada quando a onda encontra uma interface bem definida, isto é, uma região onde a diferença de impedância é a maior possível. Portanto, a maximização de band gaps naturalmente gera topologias de material discretas (RUPP et al., 2007). No entanto, a obtenção de uma topologia discreta com interfaces perfeitas entre materiais é utópica, pois há a necessidade do uso de substâncias para adesão dos materiais, como colas, por exemplo, ou geralmente há uma gradação de material existente entre as fases constituintes do material compósito provenientes, por exemplo, de processos de sinterização de compósitos.

O MOT consiste em uma abordagem robusta no projeto de materiais fonônicos. Porém, conforme descrito na Seção 1.2, o problema relaxado de otimização topológica admite soluções com valores intermediários de propriedade de material. Para se obter distribuições de material de forma discreta na célula unitária, pode-se utilizar coeficientes de penalização no modelo de material e controles de gradação de distribuição de material, como filtros, por exemplo. Entretanto, normalmente não é possível obter estruturas totalmente discretas e algumas regiões ainda continuam com valores intermediários (comumente denominadas por regiões cinzentas no MOT tradicional). Quando esses valores intermediários são interpretados na forma 0-1 no caso de aplicações fonônicas, o diagrama de dispersão pode sofrer significativas alterações e o resultado da otimização é perdido. Este efeito indesejado é exemplificado na Fig. 3.2.

A Fig. 3.2a mostra um exemplo de resultado típico da otimização topológica, onde há a presença de regiões cinzentas na distribuição de material final. No topo da figura, mostram-se as células unitárias, formadas por 40 camadas. As regiões pretas representam o PZT-5A e, as brancas, alumínio. As regiões cinzentas indicam a mistura destes dois materiais. Cada camada é representada pelos tensores das propriedades elásticas, piezelétricas e dielétricas. A célula unitária é integrante de uma matriz periódica na direção horizontal e infinita na direção vertical. Os gráficos da distribuição de material indicam as proporções das propriedades intermediárias de cada camada. Os diagramas de dispersão ilustram as regiões de propagação de onda e dos band gaps, destacadas em cinza, onde a abscissa representa as frequências e a ordenada, a parte real do vetor de onda, $k$. A parte real do vetor de onda indica onde há regiões de band gap. A Fig. 3.2b mostra 
Célula Unitária
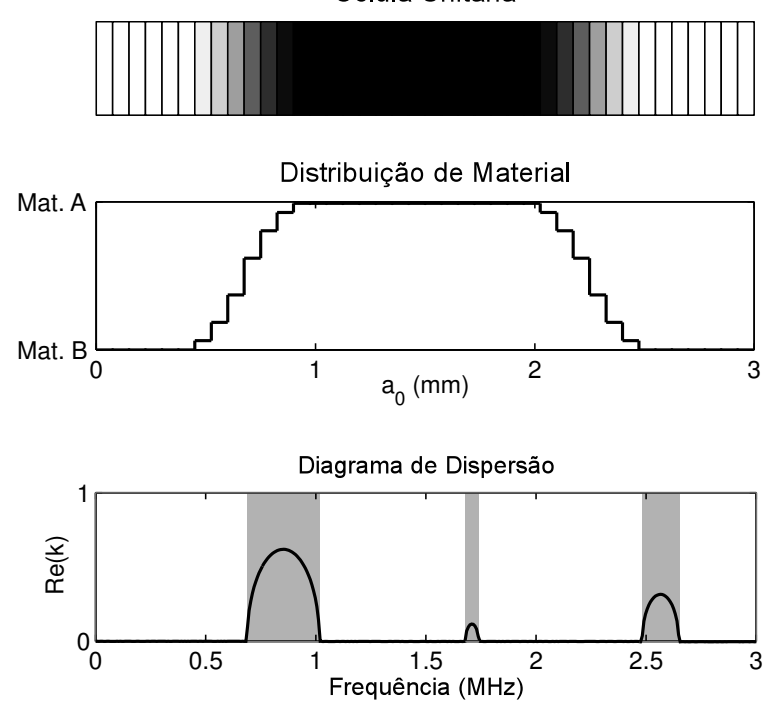

(a)

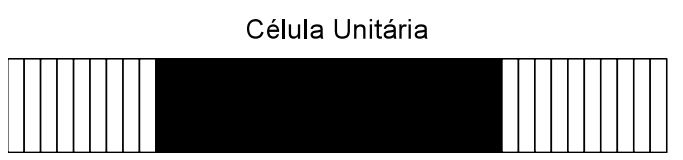

Distribuição de Material
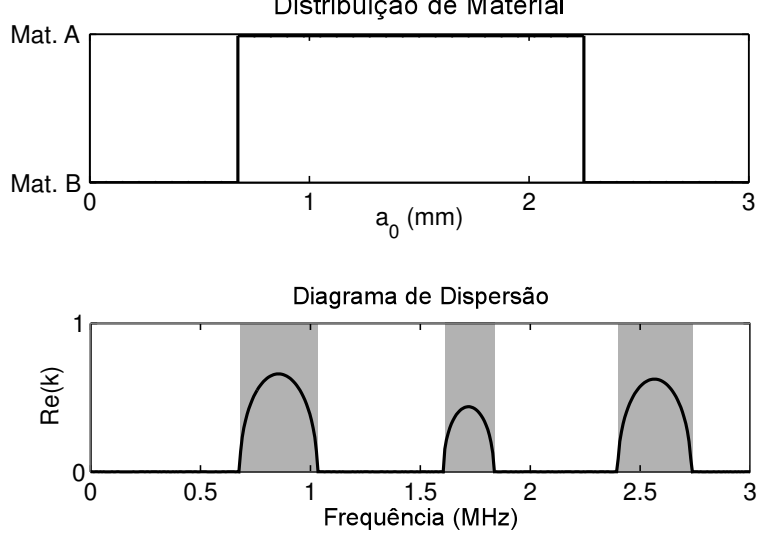

(b)

Figura 3.2: Exemplo da influência das propriedades intermediárias (regiões cinzentas) na célula unitária: (a) resultado otimizado e (b) pós-processado.

o mesmo exemplo, porém o resultado obtido da otimização é pós-processado, isto é, os valores intermediários das variáveis de projeto menores que 0,5 são projetados a 0 e, caso contrário, a 1. A Tabela 3.1 lista as posições e larguras dos band gaps para ambos os casos. Nota-se que tanto as posições quanto as larguras são modificados significativamente e o caso pós-processado apresenta band gaps mais largos. Esse aumento da largura é esperado, conforme descrito anteriormente.

Tabela 3.1: Comparação das larguras dos band gaps dos exemplos da Fig. 3.2.

\begin{tabular}{|c|c|c|c|c|c|c|}
\hline \multirow{2}{*}{ Band Gap } & \multicolumn{3}{|c|}{ Resultado otimizado $(\mathrm{MHz})$} & \multicolumn{3}{c|}{ Resultado pós-processado (MHz) } \\
\cline { 2 - 7 } & $\omega_{i}$ & $\omega_{i+1}$ & $\Delta \omega$ & $\omega_{i}$ & $\omega_{i+1}$ & $\Delta \omega$ \\
\hline $1^{o}$ & 0,690 & 1,020 & 0,330 & 0,682 & 1,035 & 0,353 \\
\hline $2^{o}$ & 1,687 & 1,748 & 0,061 & 1,612 & 1,830 & 0,218 \\
\hline $3^{o}$ & 2,480 & 2,655 & 0,175 & 2,400 & 2,738 & 0,338 \\
\hline
\end{tabular}

Assim, o pós-processamento de resultados otimizados de aplicações dinâmicas, onde o valor da função objetivo é prescrito (por exemplo, posição de um determinado band gap pré-definida), pode tornar-se extremamente difícil.

Uma forma de lidar com os problemas expostos anteriormente é utilizar o conceito de MGF no projeto de materiais fonônicos, uma vez que as regiões de propriedades intermediárias são permitidas.

Finalmente, o conceito de MGF traz outras vantagens no projeto de materiais fonônicos. Utilizando como exemplo um transdutor de ultrassom (RUBIO et al., 2009; RUBIO; SILVA; PAULINO, 2009), o conceito MGF pode contribuir na redução de reflexões nas interfaces entre materiais piezelétrico/não-piezelétrico: piezelétrico/camada-amortecimento (backing) e piezelétrico/camada-casamento 
(matching) (ver Fig. 3.3). Assim, é possível reduzir ondas indesejáveis que ecoam entre as superfícies do disco piezelétrico, permitindo o aumento da largura de banda do transdutor. Essas características são desejáveis, por exemplo, para aumentar a resolução na aquisição de imagens em aplicações de ensaios não destrutivos (END) e imagens médicas (KRAUTKRAMER J.; KRAUTKRAMER, 1977).

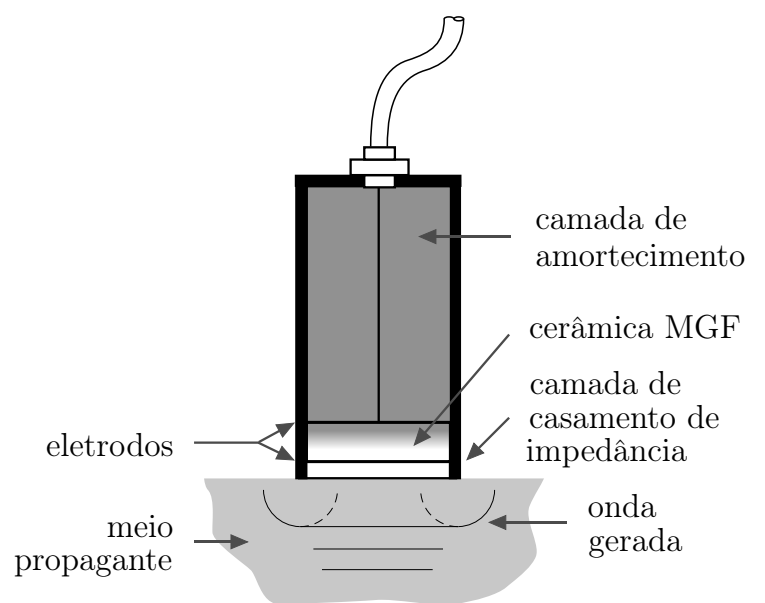

Figura 3.3: Exemplo de aplicação do conceito MGF: desenho esquemático de um transdutor piezelétrico com um piezocompósito com gradação funcional.

Por outro lado, é importante avaliar a influência de diferentes curvas de gradação de material no desempenho de materiais fonônicos, uma vez que a reflexão de ondas está diretamente relacionada com o contraste das propriedades físicas dos materiais que compõe o compósito. Esse estudo também pode ser realizado empregando-se o MOT, pois o modelo de material é relaxado, permitindo a distribuição de material com valores intermediários das propriedades, além de ser possível controlar a curva de gradação com filtros de projeção (VATANABE; SILVA, 2011b).

No caso de estruturas unidimensionais, a gradação de material pode ser obtida utilizando-se diversas camadas alternadas de materiais diferentes. Wu et al. (WU et al., 2009) investigaram a influência da gradação de material visando o aproveitamento das propriedades térmicas e mecânicas de um compósito. O estudo apresentado focou na propagação de ondas elásticas em materiais compósitos unidimensionais com gradação funcional utilizando elementos finitos espectrais e o método de matriz de transferência. Entretanto, esse estudo limitou-se a variar as propriedades dos materiais de acordo com a lei da potência, sem explorar o projeto de materiais fonônicos visando o posicionamento ou maximização dos band gaps.

Diversos trabalhos publicados até o momento concentram-se em materiais fonônicos formados por materiais isotrópicos (HLADKYHENNION; DECARPIGNY, 1991; ESQUIVELSIRVENT; COCOLETZI, 1994; LANGLET; HLADKYHENNION; DECARPIGNY, 1995; JAMES et al., 1995; SIGALAS, 1998; HALKJAER; SIGMUND; JENSEN, 2005, 2006; PHANI; 
WOODHOUSE; FLECK, 2006) e utilizando materiais piezelétricos (HOU; WU; LIU, 2004; WANG et al., 2007; ZOU et al., 2008; GONELLA; TO; LIU, 2009; WANG et al., 2009). Diversas dessas abordagens exploram modelos com formas geométricas simplificadas e limitam-se apenas em análises de sensibilidade de parâmetros geométricos. Sigmund e Jensen (SIGMUND; JENSEN, 2003) aplicaram o MOT no projeto de materiais fonônicos, a fim de minimizar as vibrações em uma determinada região (amortecimento de ondas) ou maximizá-la (guias de ondas). Rupp et al. (RUPP et al., 2007) utilizaram o MOT para projetar estruturas fonônicas 2D e 3D aplicadas a filtros de ondas de superfície e guias de onda. No entanto, nenhum trabalho visto até o momento na literatura foca no projeto de materiais piezocompósitos fonônicos com gradação de material explorado nesta pesquisa.

Uma vez que a propriedade principal dos materiais fonônicos é a presença de band gaps e como cada tipo de aplicação requer band gaps distintos, é essencial desenvolver uma metodologia robusta para projeto de materiais fonônicos, onde seja possível definir previamente parâmetros como largura e posição dos band gaps ou que maximize a largura de um determinado band gap. Assim, a motivação deste capítulo é implementar um método sistemático e genérico, como o MOT, para o projeto de materiais piezocompósitos fonônicos com gradação funcional. Duas abordagens são investigadas nesse estudo: a primeira, de modelagem unidimensional, visa projetar materiais piezocompósitos fonônicos com posição de band gaps prescrita, utilizando o Método de Matriz Em Cadeia (MMC) em conjunto ao MOT. Esse estudo tem a finalidade de explorar o conceito do problema de propagação de ondas elásticas em materiais piezocompósitos. A segunda abordagem, de modelagem bidimensional, visa o projeto de piezocompósitos com e sem gradação funcional, com o objetivo de estudar a maximização de band gaps de diversas ordens, utilizando o MOT combinado com o MEF. Nessa abordagem também é investigada a influência de diversas curvas de gradação de material no comportamento dos materiais piezocompósitos fonônicos.

Este capítulo é organizado da seguinte forma: na Seção 3.1, é brevemente apresentada a fundamentação teórica utilizada neste capítulo, que abrange o MMC, teoria de Bloch-Floquet e o MOT aplicado ao projeto de materiais fonônicos. Na Seção 3.2, é apresentada a formulação do problema de otimização topológica para band gaps prescritos em problemas unidimensionais e para maximização dos band gaps em problemas bidimensionais. Na Seção 3.3 é apresentada a implementação numérica realizada nesse estudo. Na Seção 3.4, são apresentados as verificações dos métodos utilizados e também resultados numéricos uni- e bidimensionais. Finalmente, na Seção 3.5 são listadas as conclusões obtidas neste capítulo. 


\subsection{Fundamentação Teórica}

É utilizado inicialmente um modelo analítico unidimensional a fim de explorar a física do problema de propagação de ondas elásticas em materiais compósitos. Um método unidimensional que permite a análise de materiais piezocompósitos com gradação funcional é o Método da Matriz Em Cadeia. Esse método geralmente é utilizado na literatura no projeto de transdutores piezelétricos de potência. Entretanto, esse estudo investiga a sua utilização no projeto de materiais piezocompósitos fonônicos unidimensionais. Através desse método, a distribuição de materiais num material compósito pode ser modelada através de diversas camadas enfileiradas, cada uma com propriedades diferentes. Esse método também permite realizar análises dinâmicas do material, descrevendo o seu comportamento em função da frequência de excitação. O MMC é brevemente descrito na Seção 3.1.1.

Já para estruturas bidimensionais é necessário utilizar um modelo numérico para análise. Na literatura científica, tem-se visto trabalhos de materiais fonônicos utilizando o método de Plane Wave Expansion (PWE) (WANG et al., 2007; ZOU et al., 2008; WU; WU; CHEN, 2009; WEI et al., 2009) ou o Método de Elementos Finitos Espectral (ROHAN; MIARA, 2006; ROHAN; MIARA; SEIFRT, 2009); porém, estes métodos permitem utilizar apenas inclusões de formas geométricas simples em um material base. A maior parte dos trabalhos tem utilizado o MEF (HALKJAER; SIGMUND; JENSEN, 2005, 2006; MOGAMI et al., 2006; PHANI; WOODHOUSE; FLECK, 2006; RUPP et al., 2007; DAHL; JENSEN; SIGMUND, 2008; WU; WU; CHEN, 2009), o qual permite trabalhar com distribuições de material de formas complexas na célula unitária. Assim, para projetar materiais piezocompósitos fonônicos bidimensionais é utilizado o MEF em conjunto com a teoria de Bloch-Floquet, brevemente descrito nas Seções 3.1.2 e 3.1.2. As condições de contorno de periodicidade são apresentadas na Seção 3.1.2.

\subsubsection{Modelo Unidimensional}

\section{Método de Matriz Em Cadeia (MMC)}

O Método da Matriz Em Cadeia (MMC) é um método analítico bastante eficaz utilizado para gerar uma descrição funcional dos parâmetros de impedância elétrica de diversos dispositivos de modelagem unidimensional, tais como transdutores (KRASZEWSKI et al., 1991), ou para obter campos acústicos em estruturas complexas gerados por transdutores piezelétricos de multicamadas (ADAMOWSKI et al., 1997). Uma das vantagens deste método é a possibilidade de se obter uma única matriz a qual descreve uma estrutura formada por diversas camadas acopladas, com propriedades distintas. 
A matriz em cadeia é uma matriz quadrada de dimensão 4 que define a relação de dois vetores, cada um composto por dois parâmetros acústicos e dois elétricos, onde cada vetor corresponde a uma das fronteiras transversais de uma camada de material. Neste modelo, a matriz em cadeia descreve o comportamento da camada como um todo, utilizando-se apenas das informações de ambos os vetores. Numa estrutura unidimensional, formada por diversas camadas sobrepostas, as fronteiras transversais das camadas vizinhas se coincidem e, através do "encadeamento" de diversas matrizes em cadeia, cada uma representando uma camada, é possível obter os campos acústicos e elétricos na estrutura completa.

Os elementos da matriz em cadeia são derivados a partir das equações constitutivas piezelétricas fundamentais (ver Apêndice A). O desenvolvimento da matriz em cadeia assume que o material possa ser considerado como um meio de espessura conhecida, e comprimento e largura infinitos, ou seja, como um modelo unidimensional. Assim, baseia-se na hipótese que ocorre apenas o modo de espessura, enquanto que os outros modos de vibração são desprezíveis. A formulação do MMC pode ser utilizada tanto para materiais piezelétricos como não-piezelétricos; a diferença é que as propriedades piezelétricas são nulas num material não-piezelétrico. Um diagrama da $n$-ésima camada é ilustrada na Fig. 3.4, onde $F(x)$ é a força atuante na seção transversal em $x, v(x)$ é a velocidade das partículas na posição $x, I(x)$ é a corrente de entrada na seção transversal e $V(x)$ é o potencial elétrico na seção transversal. É assumido que todas as grandezas estejam apenas em função de $x$.

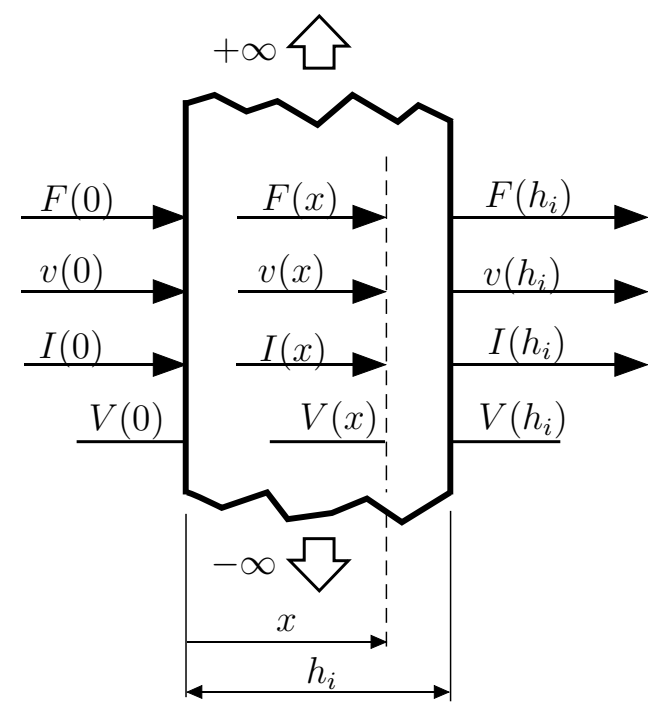

Figura 3.4: Variáveis de entrada e saída do Método da Matriz Em Cadeia.

Para ondas longitudinais propagando ao longo do eixo $x$, a matriz em cadeia relaciona as variáveis de saída força $F$, velocidade $v$, corrente elétrica $I$ e potencial elétrico $V$ no posição $x$ com os respectivos valores em $x=0$ pela equação 


$$
\left\{\begin{array}{c}
F(x) \\
v(x) \\
V(x) \\
I(x)
\end{array}\right\}=[C]\left\{\begin{array}{c}
F(0) \\
v(0) \\
V(0) \\
I(0)
\end{array}\right\}
$$

A matriz $[C]$ da Eq. (3.1) é a matriz em cadeia, dada por (KRASZEWSKI; SIGELMANN; HERBERTZ, 1991; ADAMOWSKI et al., 1997)

$$
[C]=\left[\begin{array}{cccc}
\cos (k x) & -j Z \sin (k x) & 0 & -j \beta[1-\cos (k x)] \\
-\frac{j \sin (k x)}{Z} & \cos (k x) & 0 & \frac{\beta \sin (k x)}{Z} \\
-\frac{\beta \sin (k x)}{Z} & j \beta[1-\cos (k x)] & 1 & -\left[\frac{1}{j \omega C_{0}}+\frac{j \beta^{2} \sin (k x)}{Z}\right] \\
0 & 0 & 0 & 1
\end{array}\right]
$$

onde $k$ é o número de onda, $\omega$ é a velocidade angular, $Z=A \rho c$ é a impedância acústica, $A$ é a área da seção transversal da estrutura, $\rho$ é o peso específico, $c$ é a velocidade de propagação, $C_{0}=\varepsilon^{S} A / h, \beta=e / \omega \varepsilon^{S}$, onde $e$ é o coeficiente piezelétrico, $\varepsilon^{S}$ é a constante dielétrica sob deformação constante e $h$ é a espessura da camada.

Se a célula unitária for formada por $n$ camadas, sendo que cada uma pode ter uma composição de material diferente (ver Fig. 3.5), a matriz em cadeia da camada inteira $\left([C]_{T}\right)$ é calculada como o produto da matriz em cadeia de cada camada $\left([C]_{i}\right)$, isto é, (ver Fig. 3.5) (ADAMOWSKI et al., 1997)

$$
[C]_{T}=[C]_{1}[C]_{2} \ldots[C]_{n-1}[C]_{n}=\prod_{i=1, \ldots, n}[C]_{i}
$$

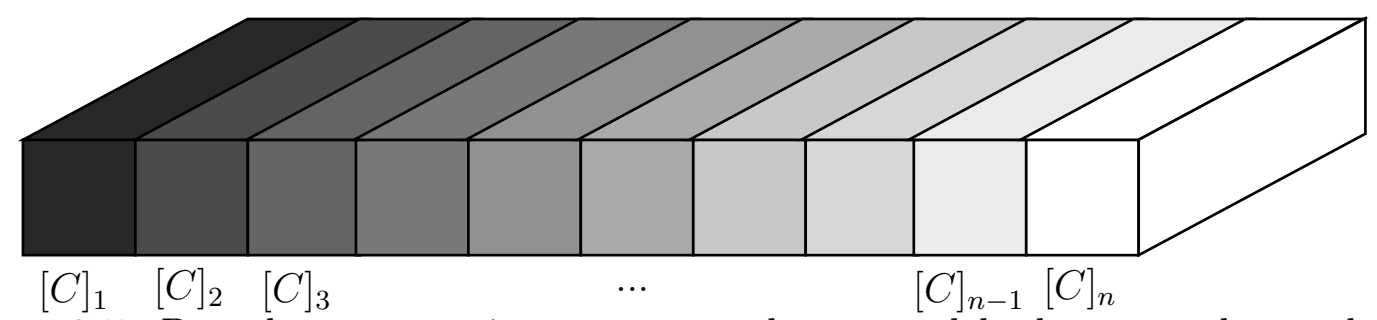

Figura 3.5: Desenho esquemático representando um modelo de $n$ camadas, onde cada uma possui uma propriedade distinta de material.

A dinâmica da propagação de onda ao longo de um material fonônico é determinada através das constantes de propagação da estrutura. A matriz em cadeia $[C]$ tem dois autovalores dependentes da frequência (RUZZENE; BAZ, 2000; TONGELE; CHEN, 2004; YEH; CHEN, 2006) dados por:

$$
\lambda_{1}=e^{\mu} \quad \text { e } \quad \lambda_{2}=e^{-\mu}
$$


onde $\mu$ é a constante de propagação. A parte real de $\mu$ representa o decaimento logarítmico da amplitude da onda propagante de uma célula unitária a outra e a parte imaginária de $\mu$ é a diferença de fase entre as células unitárias adjacentes. A propagação de ondas em estruturas periódicas ocorre quando $\mu$ possui apenas a parte imaginária e a atenuação ocorre quando $\mu$ possui parte real.

\subsubsection{Modelo Bidimensional}

\section{Teoria de Bloch-Floquet}

O segundo modelo adotado neste capítulo é de um material piezocompósito bidimensional, infinitamente periódico, assumindo o estado plano de deformações. $\mathrm{Na}$ Fig. 3.6a é mostrado um exemplo de célula unitária com uma inclusão cilíndrica numa base, cada um de materiais distintos. Devido à periodicidade do material, as bordas $A_{1} \mathrm{e}$ $A_{2}$, paralelas ao eixo $y$, e as bordas $B_{1}$ e $B_{2}$, paralelas ao eixo $x$, limitam a célula unitária, que possui dimensões $2 d_{a}$ na direção $x$ e $2 d_{b}$ na direção $y$. A célula unitária é excitada por uma onda plana monocromática, a qual é caracterizada pelo vetor de onda k, cujo módulo, k, representa o número de onda. A direção de incidência é caracterizada pelo ângulo $\theta$ em relação ao eixo $x$ positivo. A dependência do tempo é definida por $e^{-j \omega t}$. Uma vez que o material considerado é periódico e infinito nas direções $x$ e $y$, qualquer função de espaço $F$ deve satisfazer a clássica relação de Bloch (LANGLET; HLADKYHENNION; DECARPIGNY, 1995)

$$
F\left(x+2 d_{a}, y+2 d_{b}\right)=e^{j 2 d_{a} k \cos \theta} e^{j 2 d_{b} k \sin \theta} F(x, y)=e^{j \phi_{a}} e^{j \phi_{b}} F(x, y)
$$

Para se construir o diagrama de dispersão, deve-se variar a direção e comprimento do vetor de onda $\mathbf{k}$ e calcular as frequências de ressonância do modelo. À princípio, deveria-se calcular o diagrama de dispersão variando-se $\mathbf{k}$ em todas as direções da célula unitária mas, embora não tenha sido provado, a maioria dos pesquisadores alegam que é possível caracterizar uma célula unitária completamente analisando apenas a primeira zona irredutível de Brillouin (SIGMUND; JENSEN, 2003), sem perda de informações. A Fig. 3.6b mostra a primeira zona irredutível de Brillouin para uma célula unitária ortotrópica. No campo da matemática e da física dos estados sólidos, a primeira zona de Brillouin é definida unicamente pela célula primitiva do espaço recíproco. Os limites desta célula são dados pelos planos relacionados aos pontos da matriz recíproca. 


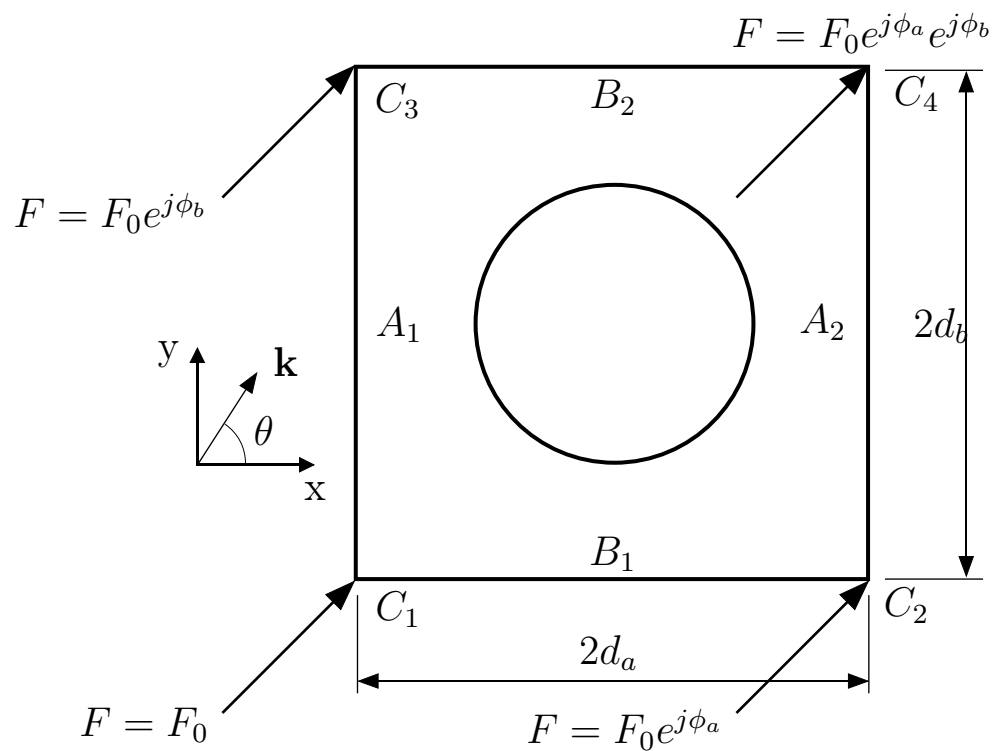

(a)

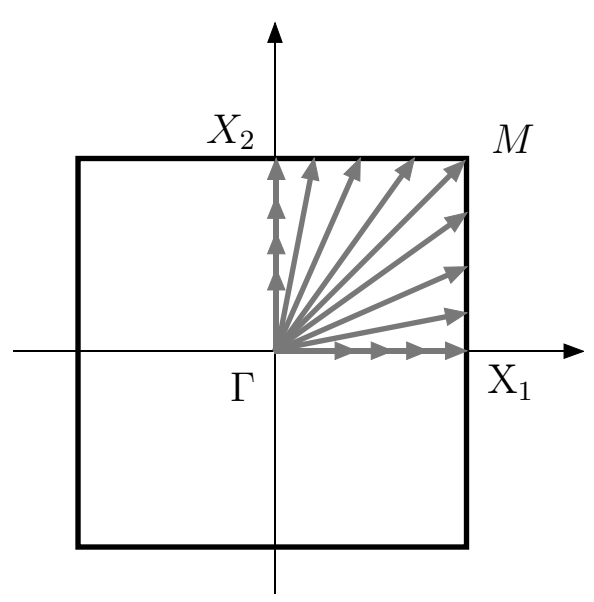

(b)

Figura 3.6: (a) Esquemático de uma célula unitária infinitamente periódica nas direções $x$ e $y$; (b) primeira zona de Brillouin para uma célula unitária ortotrópica $\left(\Gamma \rightarrow \mathrm{X}_{1} \rightarrow \mathrm{M} \rightarrow \mathrm{X}_{2} \rightarrow \Gamma\right)$.

\section{Método de Elementos Finitos (MEF) Aplicado à Estruturas Fonônicas}

No primeiro capítulo foi utilizado o MEF para aplicações estáticas. Neste capítulo, torna-se necessário implementar a formulação do MEF dinâmico com a teoria de piezeletricidade. Essa formulação é bem conhecida na literatura científica e apenas uma breve descrição é dada a seguir. Para mais informações, ver Apêndice B.

Uma vez que o material seja considerado como um meio infinitamente periódico, é possível reduzir o modelo de elementos finitos para apenas uma única célula unitária. Isto é realizado através da Eq. (3.5) nos graus de liberdade posicionados nos limites do modelo. No caso de materiais piezelétricos, existem três graus de liberdade em cada nó, os quais são deslocamentos $\mathbf{u}$ (nas direções $x$ e $y$ ) e o potencial elétrico $\phi$.

As equações de elemento finito para modelar um meio linear piezelétrico, considerando excitação harmônica, podem ser escritas da seguinte forma (NAILLON; COURSANT; BESNIER, 1983):

$$
\left(\left[\begin{array}{ll}
\mathbf{K}_{\mathbf{u u}} & \mathbf{K}_{\mathbf{u} \phi} \\
\mathbf{K}_{\mathbf{u} \phi}^{t} & \mathbf{K}_{\phi \phi}
\end{array}\right]-\omega^{2}\left[\begin{array}{cc}
\mathbf{M} & 0 \\
0 & 0
\end{array}\right]\right)\left\{\begin{array}{l}
\mathbf{U} \\
\phi
\end{array}\right\}=\left(\mathbf{K}^{*}-\omega^{2} \mathbf{M}^{*}\right) \mathbf{U}^{*}=\mathbf{0}
$$

onde $\mathbf{M}, \mathbf{K}_{\mathbf{u u}}, \mathbf{K}_{\mathbf{u} \phi}$, e $\mathbf{K}_{\phi \phi}$ são as matrizes de massa, rigidez, piezelétrica e dielétrica, respectivamente, e $\mathbf{U}$ e $\phi$ são os vetores de deslocamento e potencial elétrico nodais, respectivamente. O sistema de equações (3.6) descreve o modelo de elementos finitos para um meio piezelétrico sem considerar efeitos de amortecimento. Em todos os exemplos mostrados neste capítulo são utilizados elementos isoparamétricos, com interpolação linear 
ao longo dos lados do elemento.

\section{Condições de Contorno de Periodicidade}

As condições de contorno periódicas aplicadas nas fronteiras da célula unitária impõem que a relação de fases dos nós entre $A_{1}$ e $A_{2}$ e entre $B_{1}$ e $B_{2}$ devem ser incorporadas na equação matricial (3.6). Para isso, divide-se a célula unitária em nove partes: quatro arestas $A_{1}, A_{2}, B_{1}$, e $B_{2}$, quatro vértices $C_{1}, C_{2}, C_{3}$, e $C_{4}$, e a região interna $I$. O vetor de deslocamento $\mathbf{U}$ e o vetor de potencial elétrico $\phi$ são, portanto, divididos em nove partes, correspondentes às mesmas regiões citadas anteriormente. Devido à Eq. (3.5), tem-se que

$$
\begin{aligned}
& \mathbf{U}_{A_{2}}^{*}=\mathbf{U}_{A_{1}}^{*} e^{j \varphi_{a}}, \quad \mathbf{U}_{B_{2}}^{*}=\mathbf{U}_{B_{1}}^{*} e^{j \varphi_{b}} \quad \mathbf{U}_{C_{2}}^{*},=\mathbf{U}_{C_{1}}^{*} e^{j \varphi_{a}} \\
& \mathbf{U}_{C_{3}}^{*}=\mathbf{U}_{C_{1}}^{*} e^{j \varphi_{b}}, \quad \mathbf{U}_{C_{4}}^{*}=\mathbf{U}_{C_{1}}^{*} e^{j\left(\varphi_{a}+\varphi_{b}\right)}
\end{aligned}
$$

Pode-se definir o vetor reduzido $\mathbf{U}_{R}$, que contém os valores nodais dos deslocamentos nas arestas $A_{1}$ e $B_{1}$, no vértice $C_{1}$ e no interior do domínio, $I$. Assim, utilizando a Eq. (3.7), pode-se relacionar os vetores $\mathbf{U}$ e $\mathbf{U}_{R}$ da seguinte forma:

$$
\mathbf{U}^{*}=\mathbf{P}_{U} \mathbf{U}_{R}=\mathbf{P}_{U}\left[\begin{array}{c}
\mathbf{U}_{A_{1}}^{*} \\
\mathbf{U}_{B_{1}}^{*} \\
\mathbf{U}_{C_{1}}^{*} \\
\mathbf{U}_{I}^{*}
\end{array}\right]
$$

onde $\mathbf{P}_{U}$ tem o mesmo tamanho da matriz de rigidez $\mathbf{K}^{*}$. Em termos de elementos finitos, estas operações visam a condensação estática dos graus de liberdade pertencentes à $A_{2}$, $B_{2}, C_{2}, C_{3}$ e $C_{4}$. Assim, pode-se reescrever a Eq. (3.6) da seguinte forma:

$$
\mathbf{P}_{U}^{t}\left(\mathbf{K}^{*}-\omega^{2} \mathbf{M}^{*}\right) \mathbf{P}_{U} \mathbf{U}_{R}=\left(\mathbf{K}_{R}-\omega^{2} \mathbf{M}_{R}\right) \mathbf{U}_{R}=\mathbf{0}
$$

onde

$$
\mathbf{K}_{R}=\mathbf{P}_{U}^{t} \mathbf{K}^{*} \mathbf{P}_{U} \quad \text { e } \quad \mathbf{M}_{\mathrm{R}}=\mathbf{P}_{\mathrm{U}}^{\mathrm{t}} \mathbf{M}^{*} \mathbf{P}_{\mathrm{U}}
$$

A frequência angular $\omega$ é uma função periódica do vetor de onda k. Portanto, o problema é reduzido à primeira zona de Brillouin. Os diagramas de dispersão são obtidos através da varredura do vetor de onda $\mathbf{k}$ nas bordas da primeira zona de Brillouin. 


\subsection{Formulação do Problema de Otimização Topológica}

Como a propriedade de interesse dos materiais fonônicos é a presença de band gaps, os quais surgem devido ao contraste das propriedades dos materiais constituintes, o problema de otimização naturalmente tende a uma distribuição de material discreta. Entretanto, como esse estudo visa o projeto de materiais piezocompósitos considerando possíveis gradações entre os materiais constituintes, é necessário relaxar o problema de otimização. Assim, nesse estudo a formulação do problema de otimização utiliza um modelo de material baseado no SIMP (BENDSØE; SIGMUND, 2003), o qual define as propriedades efetivas localmente, isto é, em cada elemento finito, da seguinte forma:

$$
\begin{aligned}
\gamma^{h} & =\rho \gamma_{m_{A} t_{A}}+(1-\rho) \gamma_{m_{a t}} \\
\mathbf{c}^{h} & =\rho \mathbf{c}_{\text {mat }_{A}}+(1-\rho) \mathbf{c}_{\text {mat }_{B}} \\
\mathbf{e}^{h} & =\rho \mathbf{e}_{\text {mat }_{A}}+(1-\rho) \mathbf{e}_{\text {mat }_{B}} \\
\boldsymbol{\varepsilon}^{h} & =\rho \boldsymbol{\varepsilon}_{\text {mat }_{A}}+(1-\rho) \boldsymbol{\varepsilon}_{\text {mat }_{B}}
\end{aligned}
$$

onde $\gamma, \mathbf{c}_{\text {mat }_{i}}, \mathbf{e}_{\text {mat }_{i}}$ e $\varepsilon_{m a t_{i}}$ correspondem ao peso específico, tensores elástico, piezelétrico e dielétrico, respectivamente, dos materiais A e B. A variável $\rho$ é a pseudo-densidade que descreve a quantidade de material em cada ponto do domínio, a qual pode assumir valores entre 0 e 1 . Nesse estudo não é necessário penalizar as variáveis de projeto durante o processo de otimização pois, conforme dito anteriormente, o problema de otimização naturalmente tende a uma distribuição de material discreta.

\subsubsection{Modelo Unidimensional}

No caso de estruturas unidimensionais, a gradação de material pode ser obtida utilizando-se diversas camadas alternadas de materiais diferentes. Cada variável de projeto representa uma camada da célula unitária. Assim, o problema de otimização consiste em encontrar a melhor distribuição de material para cada camada, de forma a extremizar a função objetivo proposta. No caso do modelo unidimensional, o objetivo é obter uma topologia para a célula unitária que apresente um band gap em uma faixa de frequência prescrita. Assume-se que o meio propagante é infinitamente periódico e o domínio de projeto, que representa a célula unitária, é dividido em $n$ camadas, sendo que cada uma delas é representada por uma variável de projeto.

A formulação do problema de otimização definida para o projeto de materiais piezocompósitos fonônicos unidimensionais é dada por: 


$$
\begin{array}{ll}
\underset{\rho}{\text { Minimizar }:} & F(\rho)=\left(\omega_{1}^{c}-\omega_{1}^{p}\right)^{2}+\left(\omega_{2}^{c}-\omega_{2}^{p}\right)^{2} \\
\text { Restrito a: } & 0 \leq \rho \leq 1 \\
& \text { condições de periodicidade } \\
& \text { controle de gradação }
\end{array}
$$

onde $\omega_{1}^{p}$ e $\omega_{2}^{p}$ são o início e fim do band gap que se quer prescrever, respectivamente, $\omega_{1}^{c}$ e $\omega_{2}^{c}$ são o início e fim do band gap da estrutura calculada, respectivamente. A primeira parcela da função objetivo é aproximar os inícios do primeiro band gap, tanto do valor prescrito quanto do calculado. Da mesma forma, quer-se minimizar a diferença dos valores finais do primeiro band gap. A Fig. 3.7 ilustra a formulação do problema de otimização unidimensional através de um exemplo, onde o objetivo é posicionar as frequências calculadas $\omega_{1}^{c}$ e $\omega_{2}^{c}$ nas frequências prescritas $\omega_{1}^{p}$ e $\omega_{2}^{p}$, respectivamente.

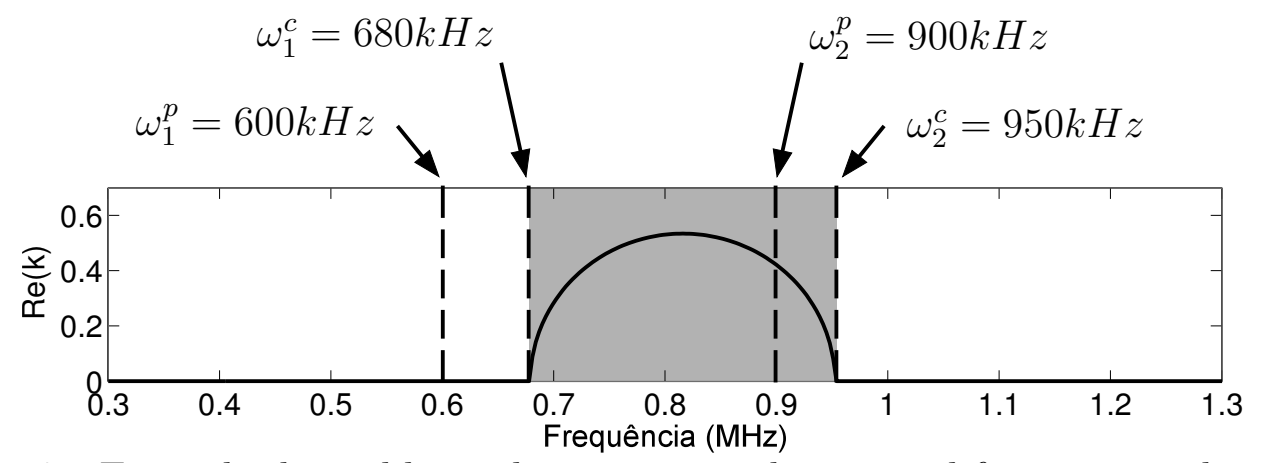

Figura 3.7: Exemplo de problema de otimização de material fonônico unidimensional.

\subsubsection{Modelo Bidimensional}

No caso do modelo bidimensional, as variáveis de projeto são distribuídas no domínio de projeto. O problema de otimização topológica consiste em encontrar a melhor distribuição de material que maximize a largura de um determinado band gap, isto é, que maximize a diferença entre dois autovalores adjacentes $\omega_{n}$ e $\omega_{n+1}$, o qual pode ser descrito por (RUPP, 2009):

$$
\begin{aligned}
\underset{d_{i}, \beta_{1}, \beta_{2}}{\operatorname{Maximizar}:} & F=\beta_{2}-\beta_{1} \\
\operatorname{restrito} a: & {\left[\mathbf{K}^{*}(\mathbf{k})-\omega^{2} \mathbf{M}^{*}\right] \mathbf{U}^{*}=\mathbf{0} \quad \mathbf{k} \in\left[\Gamma \rightarrow \mathrm{X}_{1} \rightarrow \mathrm{M} \rightarrow \mathrm{X}_{2} \rightarrow \Gamma\right] } \\
& {\left[\omega_{j+1}^{2}(\mathbf{k})\right]_{m} \geq \beta_{2} \quad m=1 \ldots n_{m} } \\
& {\left[\omega_{j}^{2}(\mathbf{k})\right]_{m} \leq \beta_{1} } \\
& 0 \leq d_{i} \leq 1 \quad i=1 \ldots N \\
& \text { controle de gradiente }
\end{aligned}
$$

onde $\omega_{j}$ é o autovalor $j$ em ordem crescente, $m$ corresponde ao vetor de onda da discretização $n_{m}$ do espaço de vetor de onda e $\beta_{1}$ e $\beta_{2}$ são variáveis de projeto 
independentes que representam individualmente os limites inferior e superior do band gap que se quer maximizar. Desta forma, não é necessário que a distribuição inicial de material já apresente um band gap pré-existente, nem que o band gap desejado sempre exista durante o processo de otimização. Assim, esta formulação faz com que o otimizador busque a melhor distribuição de material que distancie esses dois limites, mesmo que não seja possível obter um band gap na posição desejada. Além disso, o problema de inversão de modos (HAFTKA; GüRDAL, 1993) que possa ocorrer durante ou ao final da otimização não interfere na solução do problema de otimização. O controle de gradiente de material adotado no modelo bidimensional é o mesmo descrito na Seção 2.3.5.

\subsection{Implementação Numérica}

A implementação numérica dos modelos é realizada através do software MATLAB ${ }^{\circledR}$. Na abordagem unidimensional, para solução do problema analítico, calcula-se a matriz $[C]_{T}$ do modelo completo, considerando todas as camadas, e calculam-se os autovalores complexos de $[C]_{T}$ (o MATLAB ${ }^{\circledR}$ possui uma biblioteca capaz de calcular autovalores complexos). Dado os autovalores $\lambda_{1}$ e $\lambda_{2}$, calculam-se os valores de $\mu$. Construindo o gráfico da parte imaginárias de $\mu$, obtém-se o gráfico de dispersão. Para encontrar os intervalos dos band gaps, é necessário fazer uma varredura na frequência, analisando para quais valores da frequência que o vetor de onda $k$ é nulo ou igual a $\pi$.

A sensibilidade do problema unidimensional é calculada utilizando o Método de Diferenças Finitas, pois não é possível calcular analiticamente a derivada da função objetivo, uma vez que os valores de $\omega_{1}^{c}$ e $\omega_{2}^{c}$ foram obtidos via varredura da frequência e, portanto, não se tem a curva de frequência em função do vetor de onda. Finalmente, é aplicado o controle de gradação de material descrito na Seção 2.3.5 e o problema de otimização é solucionado utilizando o algoritmo MMA (Method of Moving Asymptotes) (SVANBERG, 1987).

Na abordagem bidimensional, utiliza-se o elemento quadrilátero bilinear de 4 nós devido ao custo computacional ser relativamente baixo e, portanto, pode-se trabalhar com um domínio de projeto mais discretizado. A célula unitária é dividida em 8 partes simétricas e as variáveis de projeto são distribuídas em uma dessas regiões. Entretanto, a análise é realizada considerando toda a célula unitária, pois a resposta dinâmica do sistema não é necessariamente simétrica. A direção de polarização piezelétrica é sempre definida na vertical, para toda a célula unitária. A Fig. 3.8 ilustra um exemplo do domínio de projeto e a condição de simetria adotada.

Em seguida, montam-se as matrizes de rigidez e de massa reduzidas pela Eq. (3.9) 


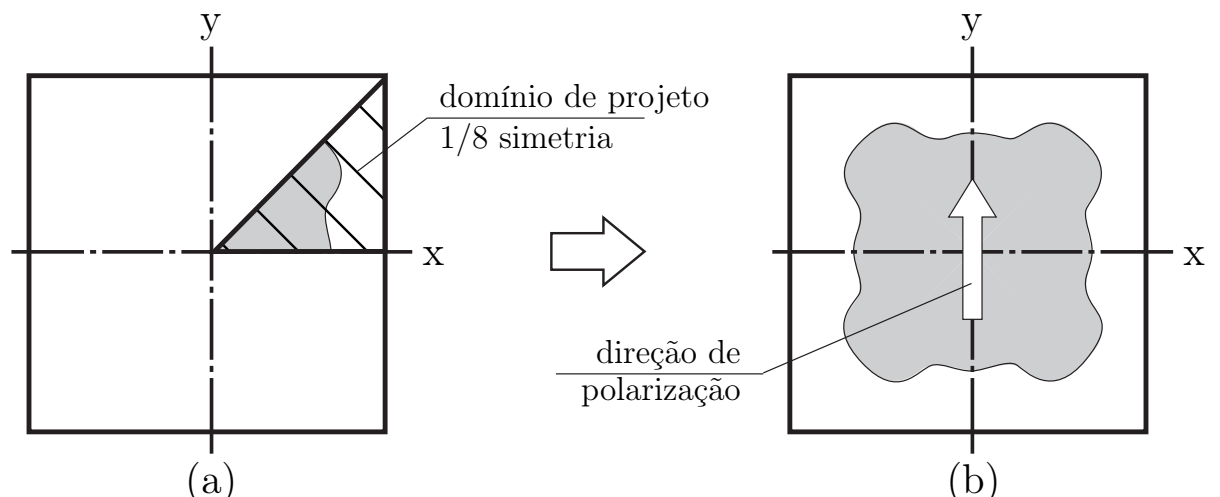

Figura 3.8: (a) Domínio de projeto alocado em $1 / 8$ da célula unitária e (b) célula unitária completa indicando a direção de polarização.

e, para cada valor do vetor de onda reduzido $\mathbf{k}$, calculam-se os autovalores através da Eq. (3.6). Dados os autovalores, constrói-se o diagrama de dispersão e avaliam-se os intervalos do primeiro band gap. A sensibilidade dos autovalores é calculada derivando a Eq. (3.8) em relação às variáveis de projeto $\rho_{i}$, cuja equação é dada da seguinte forma (SILVA; KIKUCHI, 1999):

$$
\begin{aligned}
\frac{d \omega^{2}}{d d_{i}} & =\sum_{j \in \Omega} \frac{d \omega^{2}}{d \rho_{j}} \frac{d \rho_{j}}{d d_{i}} \\
\frac{d \omega^{2}}{d \rho_{i}} & =\frac{\mathbf{U}_{R}^{t}\left(\frac{d \mathbf{K}_{R}}{d \rho_{i}}-\omega^{2} \frac{d \mathbf{M}_{R}}{d \rho_{i}}\right) \mathbf{U}_{R}}{\mathbf{U}_{R}^{t} \mathbf{M}_{R} \mathbf{U}_{R}}
\end{aligned}
$$

O controle de gradação de material é baseado na técnica descrita na Seção 2.3.5. Neste capítulo, um dos objetivos é avaliar a influência da gradação de material nos diagramas de dispersão calculados através de modelo bidimensional. Isto é realizado alterando-se o coeficiente de penalização $q$ da Eq. (2.37). Assim, dado um raio de gradação fixo $r_{\text {grad }}$, pode-se variar a forma de gradação imposta no interior da área de projeção, desde uma gradação brusca até uma gradação mais suave.

Uma vez que o domínio de projeto adotado é infinitamente periódico e, portanto, a solução do problema não é única, adota-se uma distribuição inicial aleatória de valores entre 0 e 1 para as variáveis de projeto a fim de não causar um "vício" na solução do sistema, isto é, para que a solução do problema de otimização se inicie cada vez de um ponto inicial distinto. Assim, o procedimento adotado envolve resolver o mesmo problema de otimização diversas vezes, cada vez com uma distribuição inicial diferente, até que as respostas obtidas para cada caso convirjam.

A Fig. 3.9 mostra o fluxograma do problema de otimização para o projeto de materiais piezocompósitos fonônicos. 


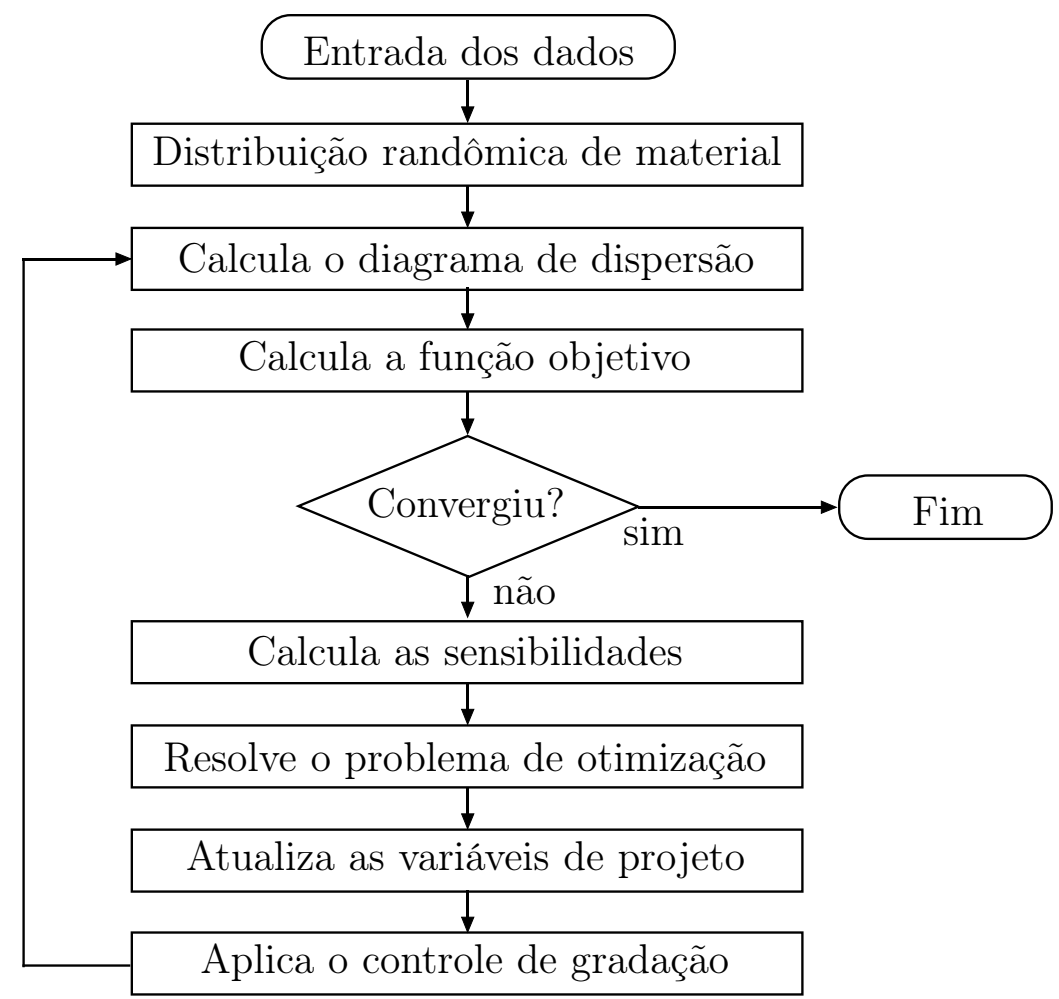

Figura 3.9: Fluxograma do problema de otimização para o projeto de materiais piezocompósitos fonônicos, tanto para o modelo unidimensional, quanto bidimensional.

\subsection{Resultados}

Nesta seção são apresentados os resultados numéricos obtidos a partir das formulações uni- e bidimensionais do problema de otimização topológica apresentado na Seção 3.2.

\subsubsection{Modelo Unidimensional}

Para o modelo unidimensional, primeiramente é realizada a verificação do modelo matemático proposto neste capítulo. Posteriormente, é avaliada a influência do efeito piezelétrico no diagrama de dispersão e, finalmente, são mostrados os resultados obtidos da otimização.

\section{Verificação do modelo implementado}

Primeiramente é realizada a verificação do modelo matemático proposto neste capítulo, através de duas formas: comparando com um modelo numérico, no caso, o método de elementos finitos, e comparando com um protótipo experimental. O modelo analítico solucionado pelo Método da Matriz Em Cadeia, adotado para verificação é mostrado na Fig. 3.10. Este modelo baseia-se numa célula unitária de duas camadas de material, uma de alumínio e outra de água. Nesse estudo, a modelagem da água é realizada como se fosse um material isotrópico. Isso é possível pois, embora a água não apresente resistência 
ao cisalhamento, no modelo unidimensional há apenas esforços de tração e compressão. As propriedades dos materiais e as dimensões do modelo estão listadas na Fig. 3.10. As propriedades são obtidas previamente através de um ensaio experimental por ultrassom. Nos modelos numéricos apresentados neste capítulo não é considerada perda, tal como amortecimento, por exemplo.

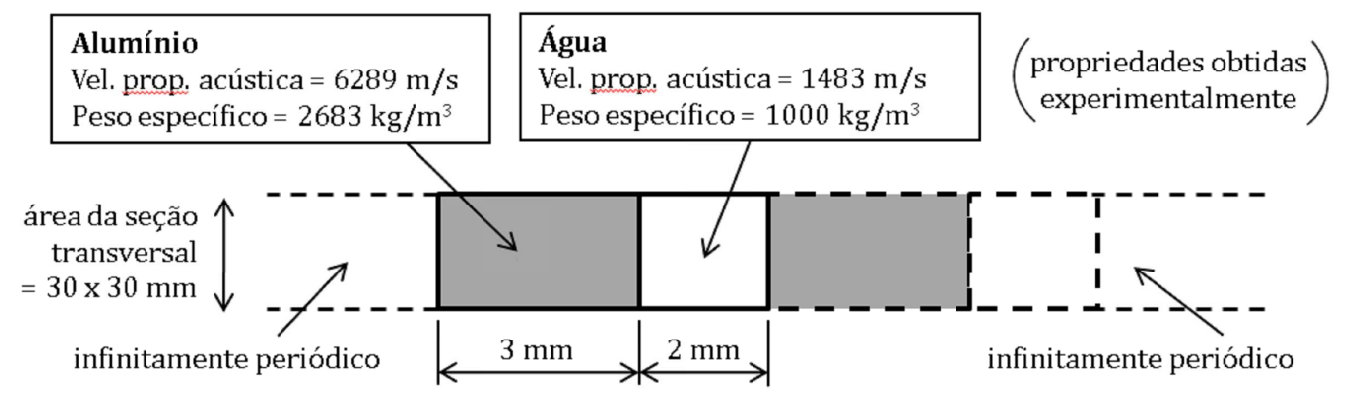

Figura 3.10: Modelo analítico utilizado para verificação do modelo numérico.

Diferentemente do modelo matemático baseado no Método da Matriz Em Cadeia, este modelo de verificação não é infinitamente periódico, a fim de se avaliar quantas células unitárias são necessárias para se obter band gaps. O modelo numérico é simulado no software ANSYS ${ }^{\circledR}$ (ver Fig. 3.11). É utilizado o elemento PLANE42, de quatro nós, e o tamanho dos elementos é de $50 \mu \mathrm{m}$. Um deslocamento prescrito harmônico, de amplitude $10 \mu m$, é imposto na face da esquerda do modelo e é medido o deslocamento na face da direita. O modelo consiste em apenas um elemento na espessura e nos nós inferior e superior do modelo são impostas condições de simetria, a fim de se avaliar apenas os efeitos unidimensionais. Considera-se que o modelo comporte-se no estado plano de deformações. O comprimento dos elementos utilizados é de 0,05 mm. A análise é do tipo harmônica e a varredura em frequência é realizada de 0 a 2 MHz, dividida em 2000 passos.

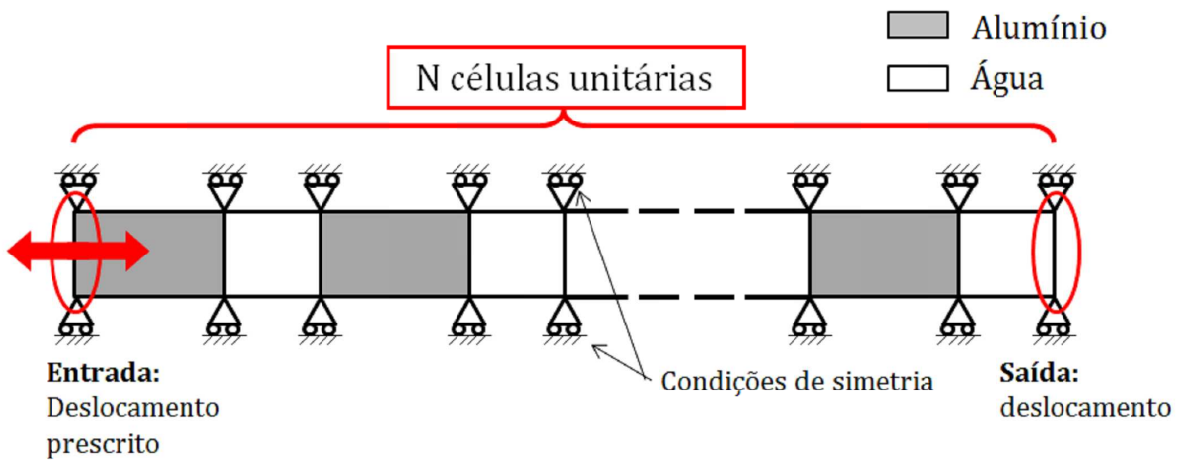

Figura 3.11: Modelo numérico utilizado para verificação do modelo.

A primeira análise a ser realizada é avaliar o número mínimo necessário de células unitárias consideradas no modelo para que haja band gaps no gráfico de dispersão. Assim, são simulados 3 casos distintos, considerando 5, 10 e 20 células unitárias. O resultado pode ser visto na Fig. 3.12. Destes diagramas é possível ver que as respostas de ambos os 
modelos são semelhantes, uma vez que a saída do modelo numérico dá-se em picos apenas nas regiões fora dos band gaps. O fato da resposta numérica ser em forma de picos, e não da forma contínua como do modelo analítico, é devido ao modelo numérico não ser infinitamente periódico. Cada pico representa uma frequência de ressonância do modelo numérico (Fig. 3.11). As condições de contorno na entrada e na saída refletem a onda propagante na estrutura, formando ondas estacionárias no seu interior. Nota-se também que o aumento de células unitárias na estrutura finita aumenta o número de frequências de ressonância nos intervalos entre os band gaps.

(a)

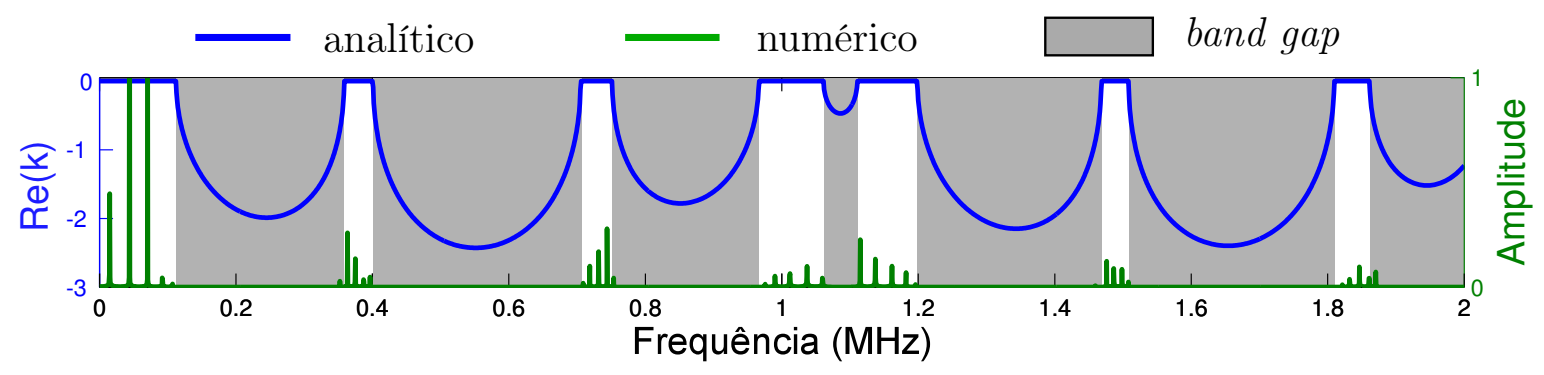

(b)

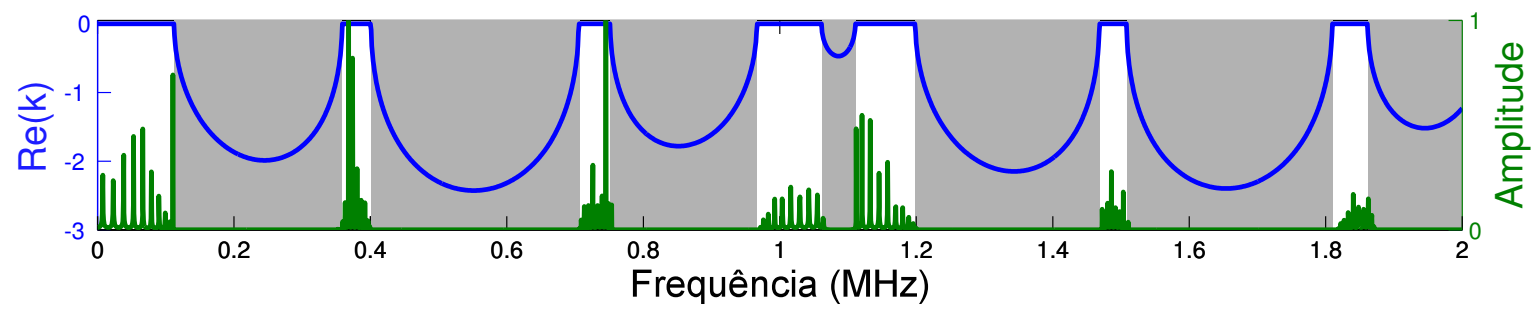

(c)

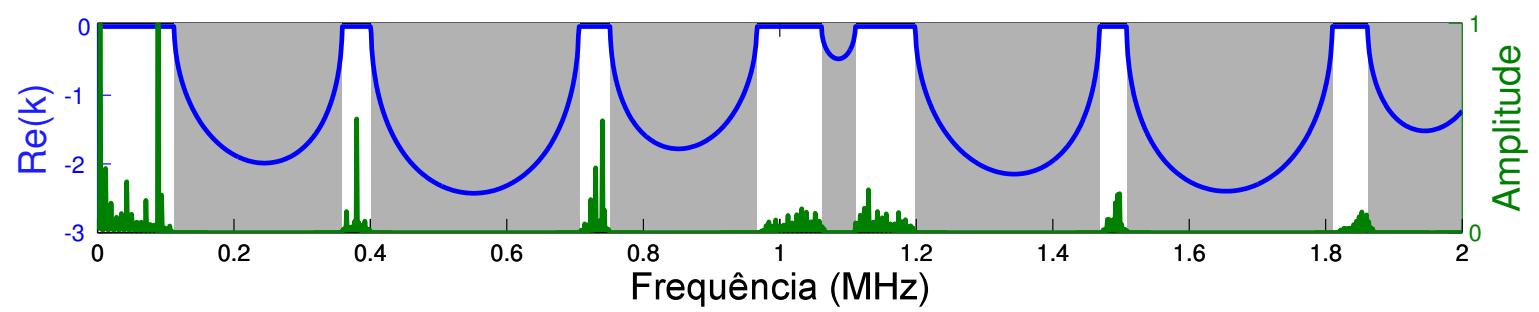

Figura 3.12: Comparação dos diagramas de dispersão dos modelos analítico e numérico, considerando: (a) 5, (b) 10 e (c) 20 células unitárias.

Como segunda forma de verificação do modelo, são realizados testes experimentais com um protótipo de alumínio submerso em água. As dimensões estão indicadas na Fig. 3.13. A Fig. 3.14a mostra o esquemático do aparato experimental e a Fig. 3.14b ilustra a medição.

O ensaio é realizado utilizando um impedômetro HP4194A. O sinal de saída considerado é obtido pela divisão do sinal do transdutor ultrassônico 2 pelo sinal do transdutor ultrassônico 1. Os transdutores utilizados são de banda larga e de frequência central em 1 e 3,4 MHz. A Fig. 3.15 mostra a curva da função transferência dos transdutores utilizados. A unidade da amplitude é arbitrária, uma vez que o interesse nesse estudo é o comportamento do material.

A Fig. 3.16 compara o gráfico de dispersão do modelo analítico com o resultado 


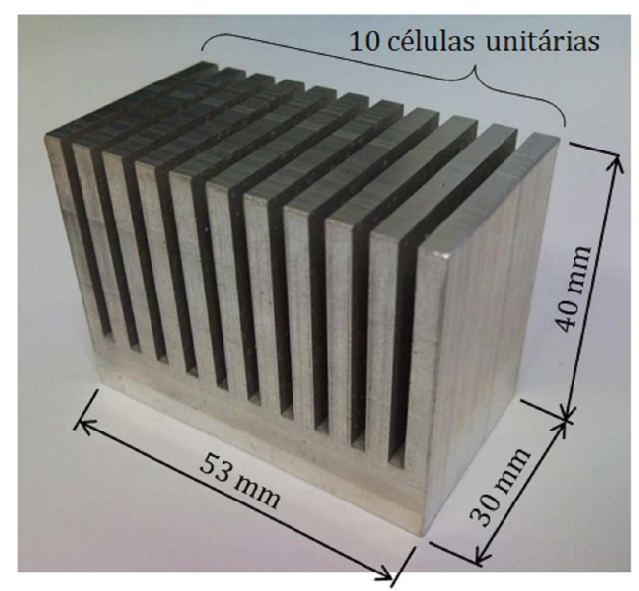

Figura 3.13: Dimensões do protótipo fabricado, utilizado para verificação do modelo.

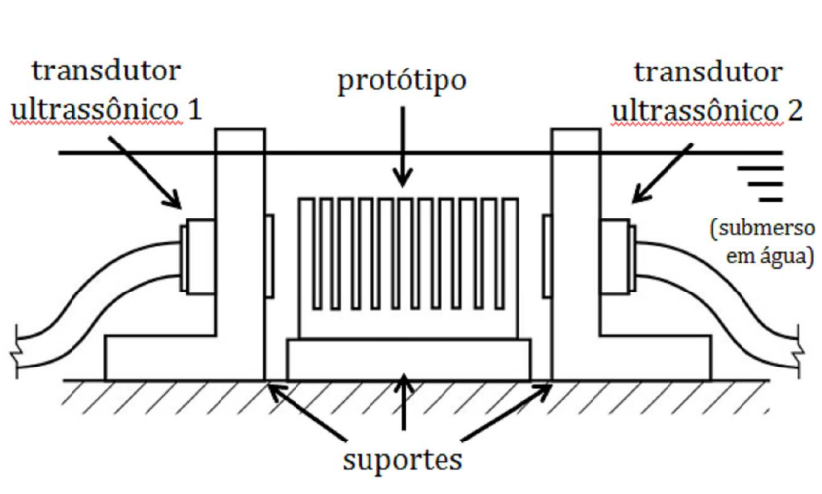

(a)

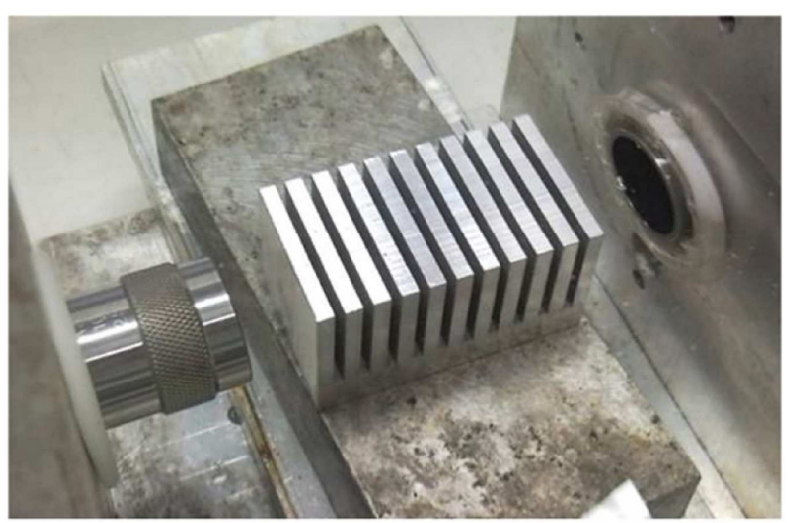

(b)

Figura 3.14: (a) Desenho esquemático do aparato experimental; (b) imagem do ensaio experimental.

experimental. O sinal de saída medido experimentalmente corresponde à amplitude da onda na face de saída do protótipo, normalizado em relação ao seu valor máximo para facilitar a visualização. Nesse gráfico, nota-se que a região entre 1 e 1,2 MHz há a propagação de ondas no experimento, ao contrário do que indica o resultado analítico. Isso pode ser explicado por três motivos. O primeiro é que justamente nessa região a sensibilidade dos transdutores utilizados é maior, como pode ser visto na Fig. 3.15. Além disso, é sabido que a intensidade da atenuação da onda incidente nos band gaps é exponencialmente relacionada ao valor imaginário do vetor de onda (HUSSEIN et al., 2006). Portanto, a onda propagante não pode ser totalmente atenuada nessa região entre 1 e 1,2 MHz. Para sanar esse problema, uma solução é aumentar o número de células unitárias. Uma terceira justificativa para a ocorrência deste fenômeno é a transmissão da onda através da base do protótipo, isto é, onde os rasgos usinados no alumínio terminam.

Da Fig. 3.16, nota-se que o resultado experimental também se dá na forma de picos, semelhante ao resultado numérico, devido ao modelo não ser infinitamente periódico. A concordância do sinal medido com o modelo analítico é bastante próxima, principalmente para baixas frequências. A amplitude do sinal medido é maior na região de $1 \mathrm{MHz}$, a 


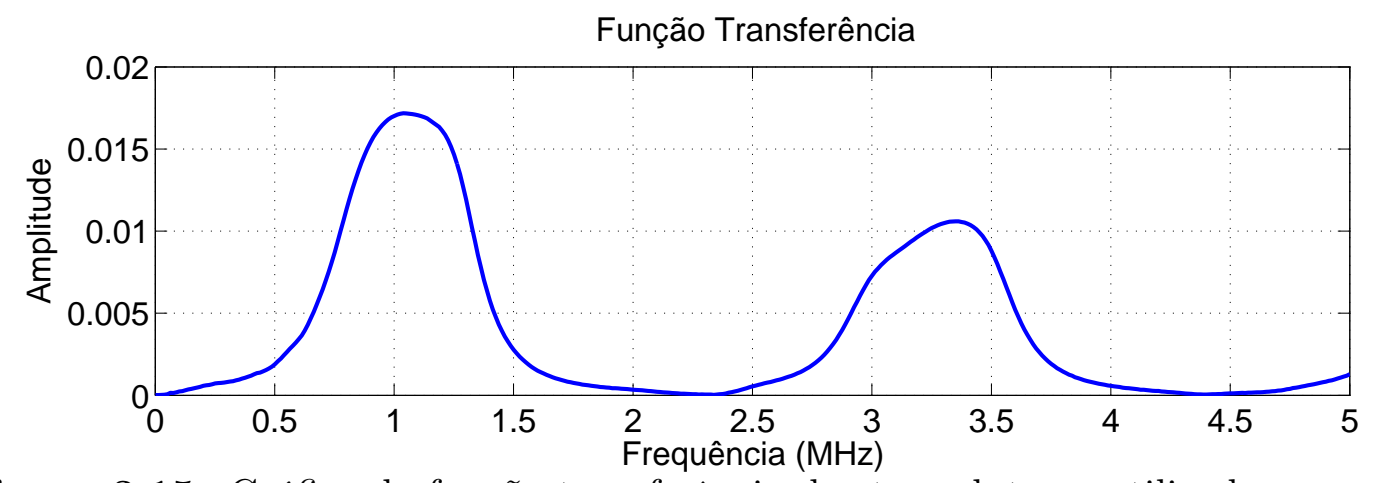

Figura 3.15: Gráfico da função transferência dos transdutores utilizados nos ensaios experimentais.
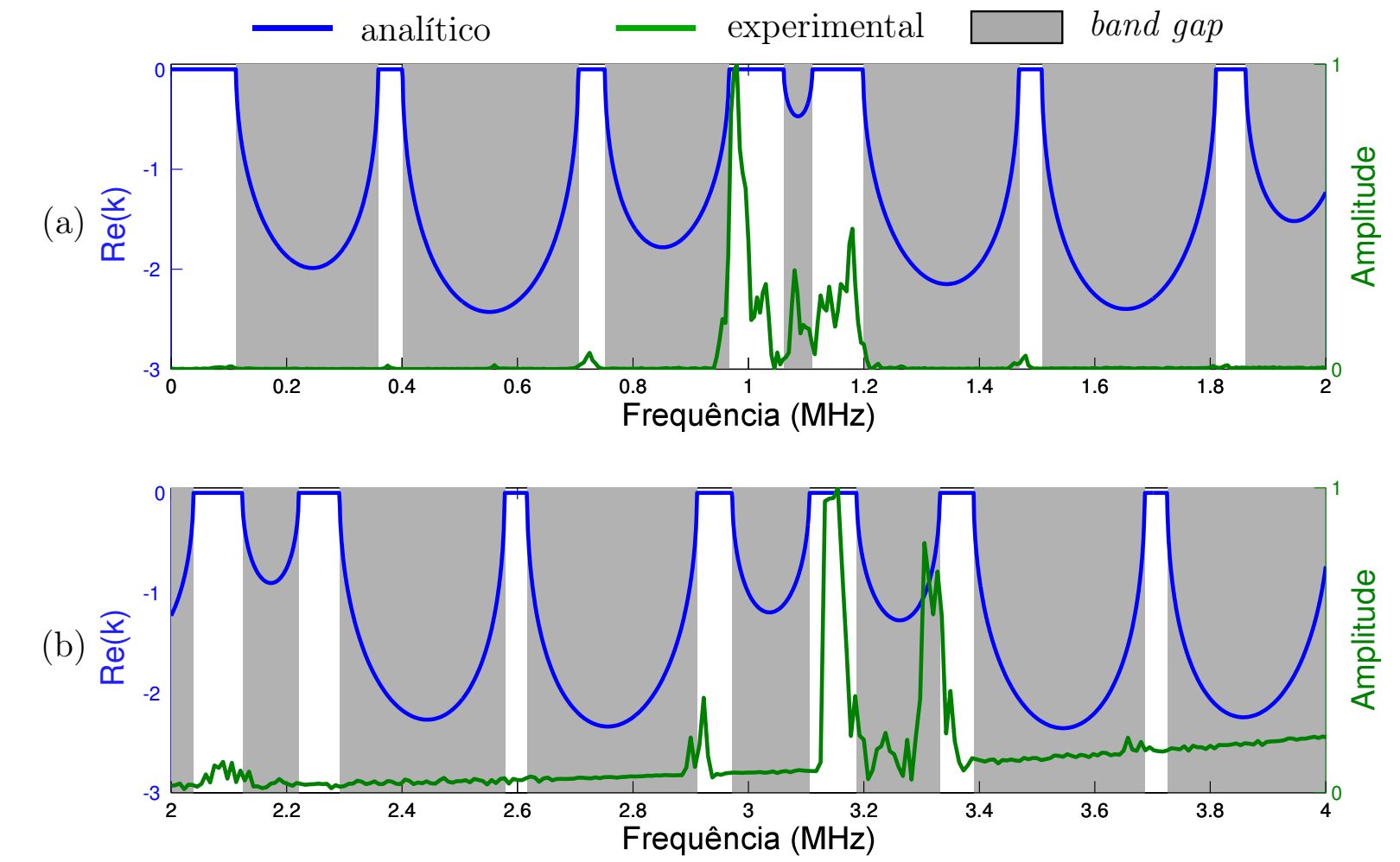

Figura 3.16: Comparação dos diagramas de dispersão do modelo analítico e medido experimentalmente: intervalos de frequência (a) de 0 a $2 \mathrm{MHz}$ e (b) de 2 a $4 \mathrm{MHz}$.

mesma da frequência central do transdutor.

A partir dos diagramas de dispersão mostrados nas Fig. 3.12 e 3.16, conclui-se que o modelo proposto neste trabalho é válido.

\section{Influência do efeito piezelétrico}

Para verificar a influência do efeito piezelétrico no diagrama de dispersão, primeiramente é calculado o desempenho de uma célula unitária composta por camadas alternadas de PZT-5A e alumínio. As propriedades dos materiais utilizadas estão listadas na Tabela 3.2 (a polarização do PZT-5A é considerada na vertical). A fração de volume adotada de PZT-5A é de 0,5, conforme mostrado na Fig. 3.17. São analisados dois casos, um com propriedades piezelétricas iguais as da Tabela 3.2 e outro com propriedades piezelétricas 
nulas.

Tabela 3.2: Tabela de propriedades do material PZT-5A e do alumínio utilizadas no modelo unidimensional.

\begin{tabular}{|c|c|c|}
\hline Propriedade & PZT-5A & Alumínio \\
\hline$c_{33}^{E}\left(10^{10} \mathrm{~N} / \mathrm{m}^{2}\right)$ & 11,1 & 108,2 \\
\hline$e_{33}\left(C / \mathrm{m}^{2}\right)$ & 15,8 & 0 \\
\hline$\varepsilon_{33}^{S} / \varepsilon_{0}$ & 1700 & 8,9 \\
\hline Peso específico $\left(\mathrm{kg} / \mathrm{m}^{3}\right)$ & 7500 & 2700 \\
\hline
\end{tabular}

Célula Unitária

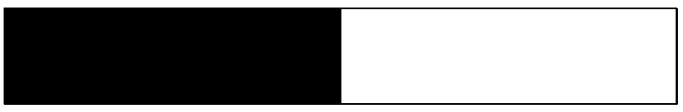

(a)

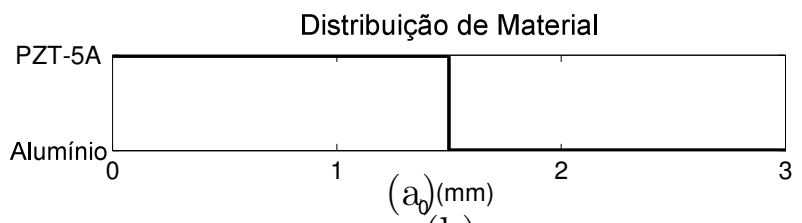

(b)

Figura 3.17: Exemplo do influência do efeito piezelétrico na célula unitária com camadas alternadas de PZT-5A e alumínio: (a) célula unitária e (b) distribuição de material.

Os diagramas de dispersão para ambos os casos são mostrados na Fig. 3.18, onde é possível notar que as larguras do band gap obtidos com propriedades piezelétricas nulas são menores que as obtidas com propriedades piezelétricas não nulas (ver Tabela 3.3). Este exemplo mostra que a presença das propriedades piezelétricas pode contribuir na obtenção de band gaps maiores.

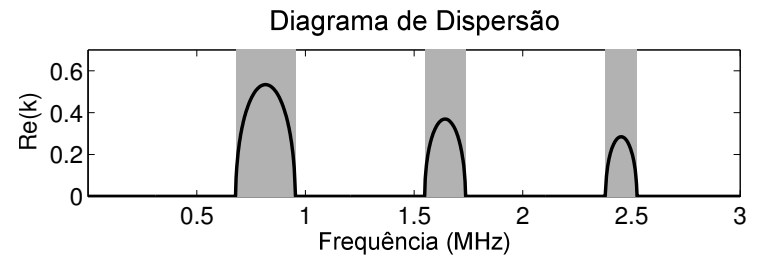

(a) não polarizado

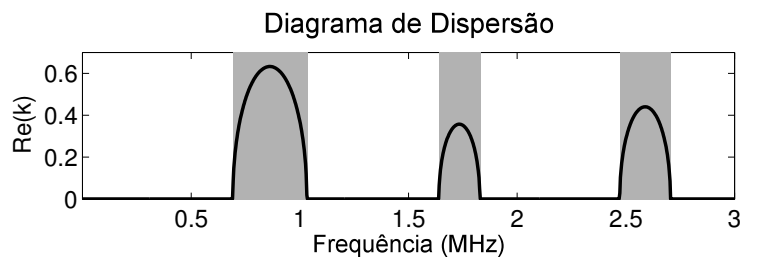

(b) polarizado

Figura 3.18: Diagramas de dispersão mostrando a influência do efeito piezelétrico na célula unitária com camadas alternadas de PZT-5A e alumínio.

\section{Projeto de materiais fonônicos gradados com band gaps prescritos}

O projeto de materiais fonônicos visando a posição e largura dos band gaps é possível de ser realizado através da variação da proporção entre os materiais constituintes em cada camada. Para isso, cada camada de material da célula unitária é representada por uma variável de projeto. Embora um grande número de camadas na célula unitária permita obter distribuições de material mais complexas e, consequentemente, um controle maior do comportamento do diagrama de dispersão, isso gera também um aumento no custo computacional e de manufatura. Portanto, nos exemplos mostrados a seguir adotam-se 40 camadas no domínio de projeto. A distribuição inicial de material é gerada de forma aleatória. O raio de projeção adotado nestes exemplos é fixado em $r_{\text {grad }}=10 \%$, a fim de 
Tabela 3.3: Largura dos band gaps obtidos com e sem polarização piezelétrica.

\begin{tabular}{|c|c|c|}
\hline Band gap & $\begin{array}{c}\text { PZT-5A } \\
\text { não polarizado }(\mathrm{kHz})\end{array}$ & $\begin{array}{c}\text { PZT-5A } \\
\text { polarizado }(\mathrm{kHz})\end{array}$ \\
\hline $1^{o}$ & 273 & 342 \\
\hline $2^{o}$ & 186 & 189 \\
\hline $3^{o}$ & 144 & 234 \\
\hline
\end{tabular}

se obter uma gradação suave na distribuição de material na célula unitária. O intervalo de frequências analisado varia de 0 a $2 \mathrm{MHz}$, dividido em 400 passos.

Os exemplos mostrados nessa seção combinam os materiais PZT-5A e alumínio. As propriedades dos materiais utilizadas estão listadas na Tabela 3.2 (a polarização do PZT-5A considerada é na vertical). São simulados seis casos para mostrar a viabilidade do projeto de materiais piezocompósitos fonônicos com prescrição de band gap. Para os quatro primeiros casos são definidas as seguintes frequências para o primeiro band gap: de 400 a $500 \mathrm{kHz}$, de 500 a $600 \mathrm{kHz}$, de 600 a $700 \mathrm{kHz}$ e de $700 \mathrm{kHz}$ a $800 \mathrm{kHz}$. Os comprimentos da célula unitária adotado para esses quatro primeiros casos são: $5,5 \mathrm{~mm}$, 4,5 mm, 3,9 mm e 3,3 mm, respectivamente. Os objetivos do quinto e sexto casos é posicionar o terceiro band gap entre 1,5 e 1,6 $\mathrm{MHz}$ e 1,6 e 1,7 MHz, respectivamente, com células unitárias de 5,5 e 4,6 mm de comprimento, respectivamente. O critério de convergência considerado em todos os casos é um desvio menor do que $5 \mathrm{kHz}$ na função objetivo. As Fig. 3.19 a 3.21 mostram as células unitárias otimizadas para cada caso, incluindo a distribuição de material nas camadas ao longo da célula unitária e os diagramas de dispersão. Os quatro primeiros casos apresentam uma curva de gradação suave ao longo da célula unitária, enquanto que a gradação de material do quinto e sexto caso são mais oscilatórias. Apenas o primeiro exemplo apresenta regiões de PZT-5A puro, enquanto que nos outros casos sempre há uma mistura dos materiais constituintes. É possível observar também a condição de periodicidade imposta no projeto através das curvas de distribuição de material contínuas nas bordas das células unitárias. As soluções apresentadas para cada caso não são únicas pois, por serem estruturas periódicas, quaisquer intervalos de comprimento da célula unitária apresentarão os mesmos resultados.

\subsubsection{Modelo Bidimensional}

Esta seção explora o projeto de materiais piezocompósitos fonônicos bidimensionais utilizando o MOT. Primeiramente é realizada a verificação do modelo numérico implementado, através da comparação com resultado disponível na literatura. Então, é mostrada a influência do efeito piezelétrico em materiais piezocompósitos fonônicos bidimensionais. Em seguida, é apresentado o projeto de diversas células unitárias visando 

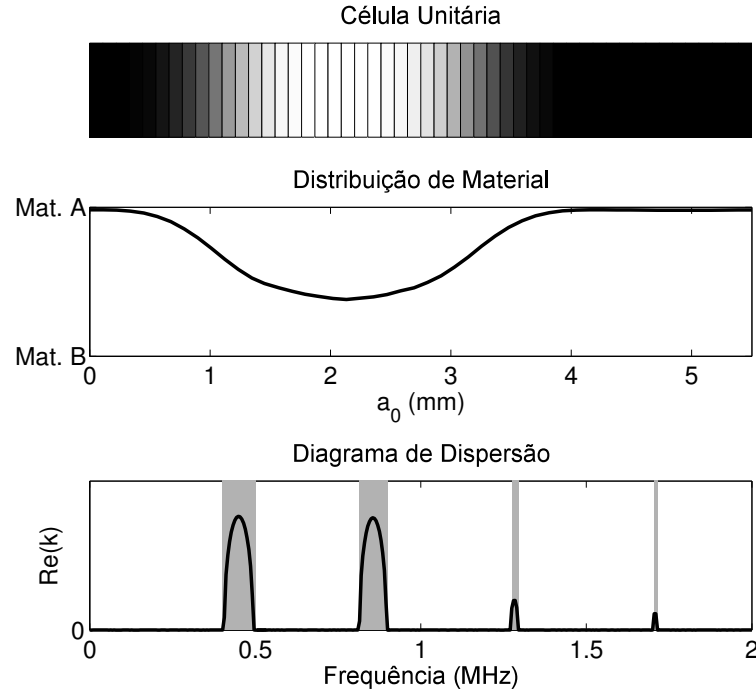

(a)

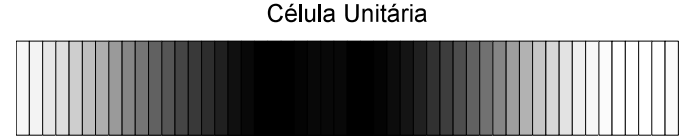

Distribuição de Material
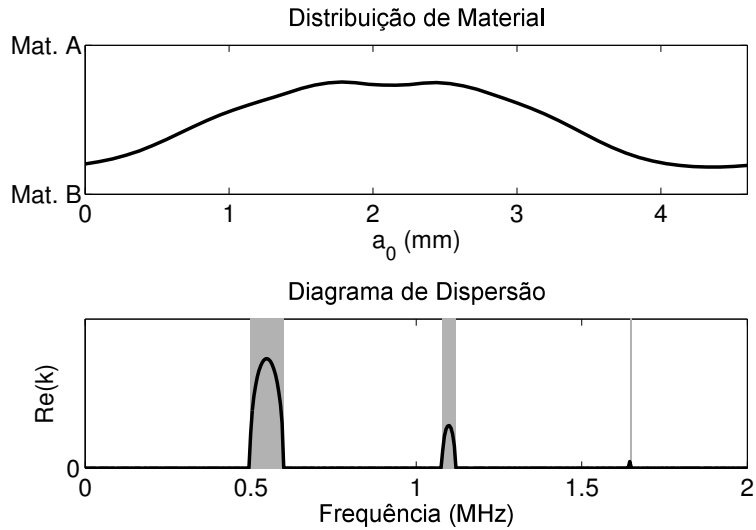

(b)

Figura 3.19: Resultado otimizado com primeiro band gap prescrito entre (a) 400 a 500 kHz e (b) 500 a $600 \mathrm{kHz}$.

Célula Unitária
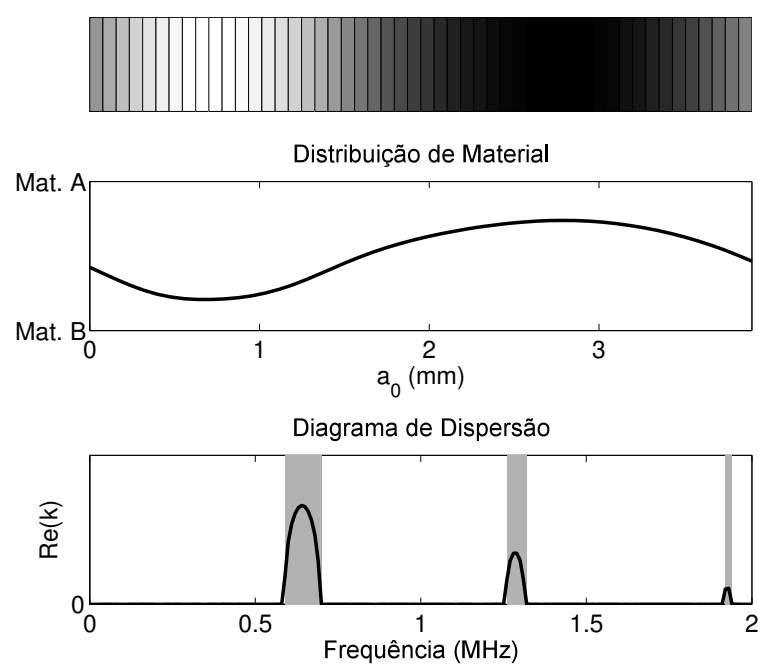

(a)

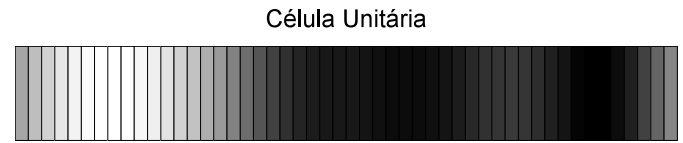

Distribuição de Material
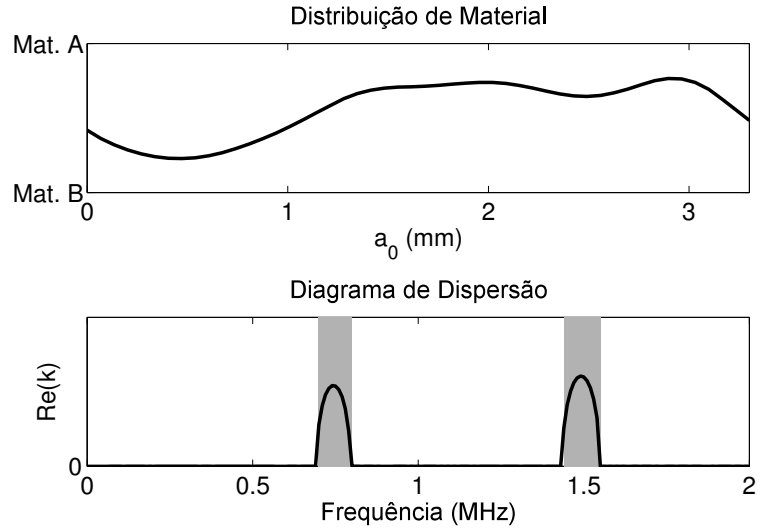

(b)

Figura 3.20: Resultado otimizado com primeiro band gap prescrito entre (a) 600 a 700 kHz e (b) 700 a 800 kHz. 
Célula Unitária
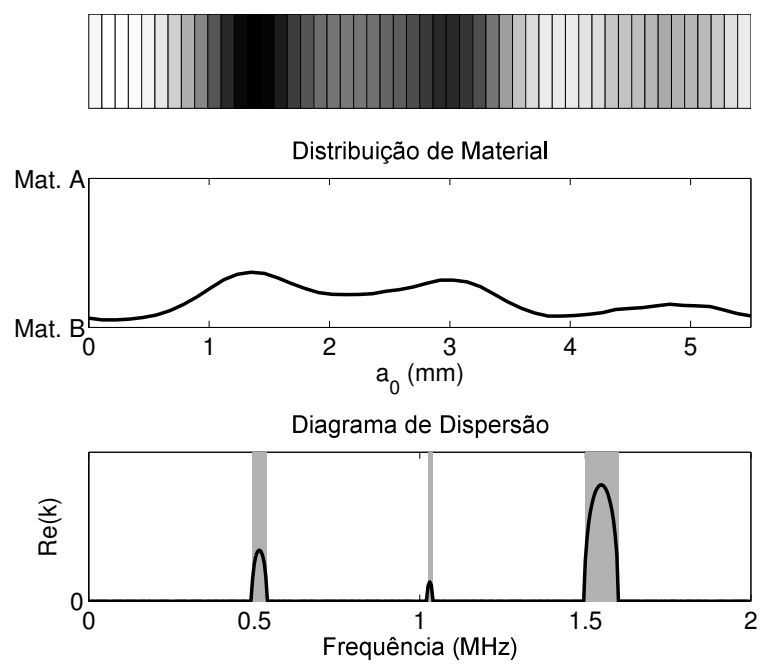

(a)
Célula Unitária
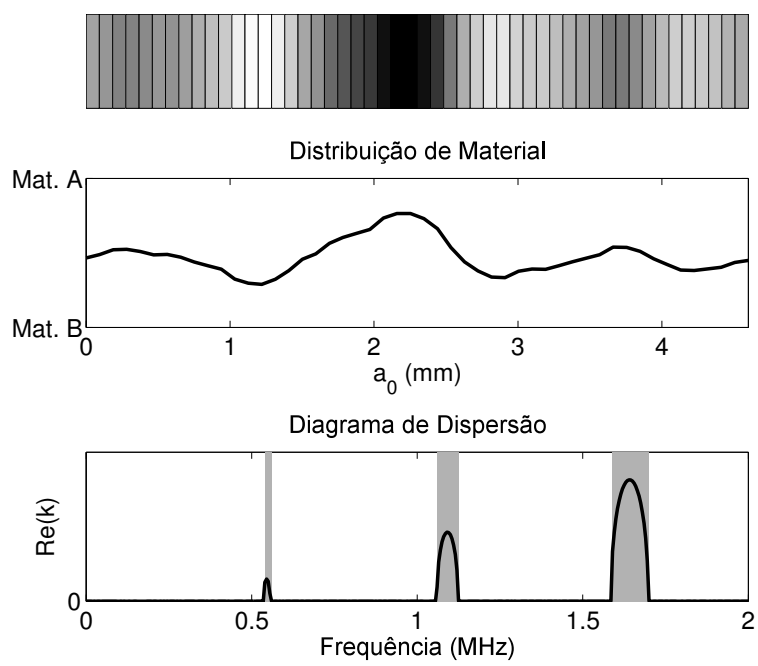

(b)

Figura 3.21: Resultado otimizado com terceiro band gap prescrito entre (a) 1,5 a 1,6 $\mathrm{MHz}$ e (b) 1,6 a $1,7 \mathrm{kHz}$.

a maximização de band gaps de diversas ordens. Os materiais utilizados nestas análises são a cerâmica piezelétrica PZT-5A e o polímero epóxi, cujas propriedades estão listadas na Tabela 2.4. Ressalta-se que nesses exemplos, a direção de polarização piezelétrica é fixada na direção $y$. Por fim, é verificada a influência de diferentes curvas de gradação de material nos materiais piezocompósitos fonônicos bidimensionais alterando-se os valores do coeficiente de penalização $q$.

\section{Verificação do modelo implementado}

A verificação da implementação numérica pode ser realizada comparando-se os resultados do MEF com um modelo analítico da literatura. Para isso, é calculado o diagrama de dispersão para um modelo isotrópico elástico, homogêneo, infinitamente periódico e assumindo o estado plano de deformações. O material adotado é alumínio, cujas propriedades consideradas são $E=73,1 G P a, \nu=0,325$ e $\rho=2770 \mathrm{kgm}^{-3}$. O modelo é um quadrado com comprimento de aresta igual a $2 d=4 \mathrm{~cm}$, discretizado por $20 \times 20$ elementos quadriláteros bilineares. O vetor de onda $\mathbf{k}$ analisado é o referente ao primeiro triângulo de Brillouin. O modelo analítico pode ser calculado através da equação (LANGLET; HLADKYHENNION; DECARPIGNY, 1995):

$$
\omega=c \sqrt{\left(k_{x}+n \pi / d\right)^{2}+\left(k_{y}+m \pi / d\right)^{2}}
$$

onde $n$ e $m$ são inteiros, $c$ é a velocidade de propagação longitudinal $c_{L}$ ou transversal $c_{T}$, e $k_{x}$ e $k_{y}$ são as componentes do vetor de onda $k$ projetados nos eixos $x$ e $y$.

A Fig. 3.22 mostra o diagrama de dispersão para o modelo de elementos finitos e 


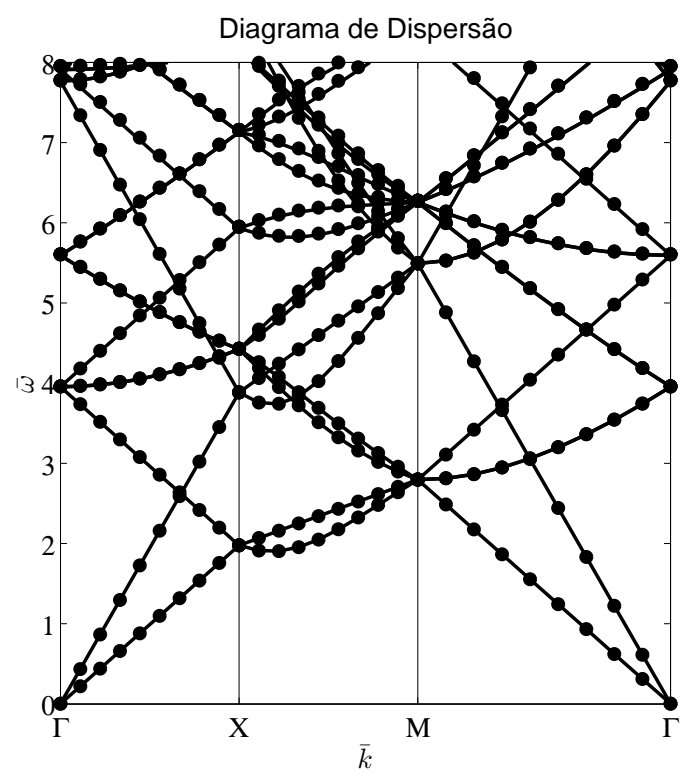

Figura 3.22: Verificação do modelo de elementos finitos bidimensional implementado com um modelo analítico (linhas: analítico; pontos: resultados do MEF).

analítico, utilizando a frequência angular reduzida $\bar{\omega}=2 d \omega \sqrt{E / 3(1-2 \nu)}$ em função do vetor de onda reduzido $\bar{k}=2 d k / \pi$. Do gráfico, nota-se a concordância dos resultados numéricos com o analítico.

O modelo numérico bidimensional implementado também é verificado com um exemplo disponível na literatura (LANGLET; HLADKYHENNION; DECARPIGNY, 1995), o qual consiste em uma matriz de PVC $\left(E=3,78 G P a, \nu=0,4, \gamma=1430 \mathrm{kgm}^{-3}\right)$ com um furo circular periódico (ver modelo discretizado na Fig. 3.23a). A dimensão do furo em relação ao comprimento da célula unitária é de $a / d=0,8$. O diagrama de dispersão calculado utilizando o modelo numérico implementado pode ser visto na Fig. 3.23b, onde é considerado apenas a propagação de ondas na direção do eixo $y$. Deste gráfico, é possível ver um band gap entre as frequências normalizadas 0,2638 e 0,5313, coincidindo com o resultado apresentado por Langlet, Hladkyhennion e Decarpigny (1995).

\section{Influência do efeito piezelétrico}

É verificada novamente a influência do efeito piezelétrico no diagrama de dispersão, porém agora utilizando um exemplo bidimensional. São analisados dois exemplos de distribuição de epóxi e PZT-5A, sendo que um dos exemplos tem propriedades piezelétricas iguais às da Tabela 2.4 e outro com propriedades piezelétricas nulas. A fração de volume adotada de PZT-5A é de 0,5. Os diagramas de dispersão para ambos os casos são mostrados na Fig. 3.24. O eixo das ordenadas representa a frequência normalizada $\omega a_{0} / 2 \pi c_{A}$, onde $a_{0}$ é a largura da célula unitária e $c_{A}$ é a velocidade de propagação do material $\mathrm{A}\left(\sqrt{E_{A} / \rho_{A}}\right)$. É possível notar que a largura do band gap obtido com propriedades piezelétricas nulas $(\Delta \omega=0,4283)$ é menor que a obtida com propriedades 


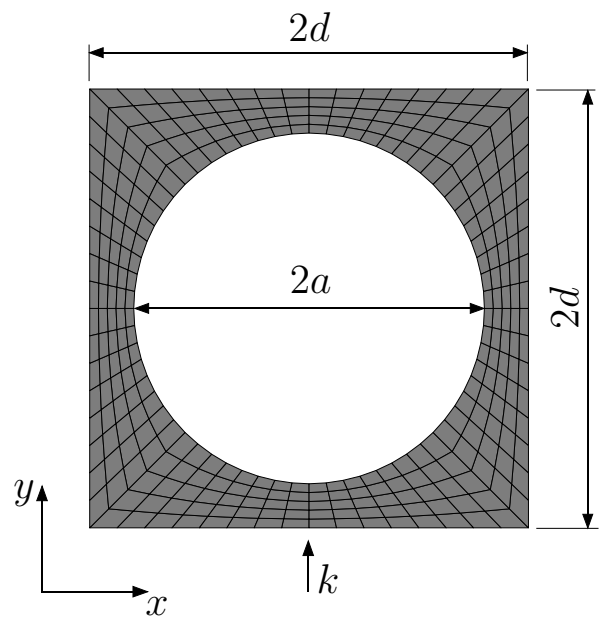

(a)

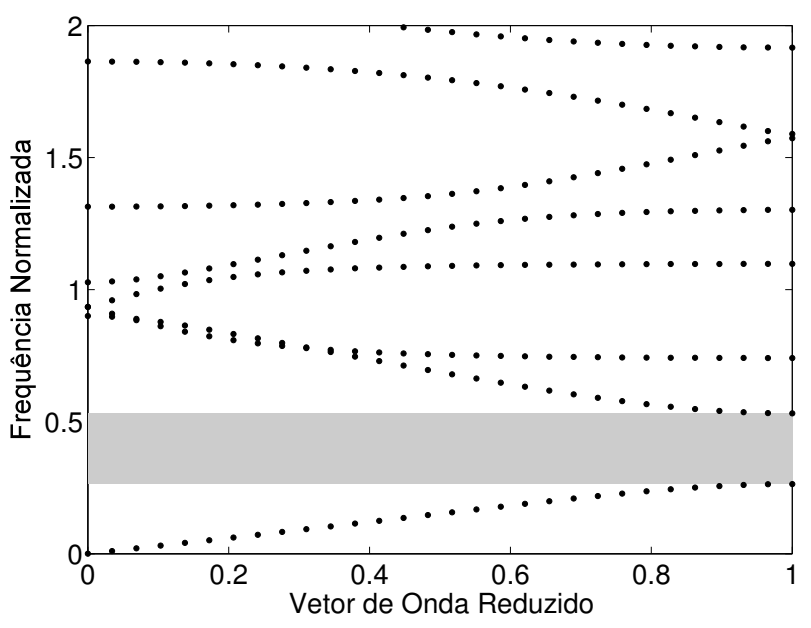

(b)

Figura 3.23: Verificação do modelo numérico implementado: (a) célula unitária de PVC com furo cilíndrico e (b) diagrama de dispersão (cinza: band gap).

piezelétricas não nulas $(\Delta \omega=0,4916)$, representando um aumento de $15 \%$ na largura do band gap. Semelhante ao caso unidimensional, este exemplo mostra que a presença das propriedades piezelétricas pode contribuir na obtenção de band gaps maiores.

\section{Maximização de band gaps}

Nesta seção são apresentados os resultados de materiais piezocompósitos fonônicos considerando a maximização de band gaps de diversas ordens. A célula unitária é discretizada em $60 \times 60$ elementos (três graus de liberdade por nó, sendo dois de deslocamento e um de potencial elétrico). O raio da projeção $r_{\min }$ considerado é igual a $5 \%$ da célula unitária e é adotado o valor do coeficiente da projeção igual a $q=8$, a fim de se obter uma topologia final discreta. São maximizados dez band gaps como exemplo, mostrados a seguir.

O primeiro exemplo visa a maximização do band gap entre a $3^{a}$ e $4^{a}$ frequência, resultando numa largura normalizada igual a 0,5312 (ver Fig. 3.25a), e a distribuição de PZT-5A obtida assume a forma de octógono. Nota-se a presença de inclusões de PZT na matriz de epóxi como esperado nos resultados mostrados na literatura (RUPP et al., 2007). Interpretando esse resultado para uma topologia discreta, isto é, assumindo que todas as variáveis de projeto acima de um determinado valor (por exemplo, $d \geq 0,5$ ) sejam igual a 1 e os outros igual a 0, a largura do band gap aumenta para 0,5454 (ganho de 2,7\% em relação ao resultado não interpretado), mostrado na Fig. 3.25b. Conforme discutido anteriormente, quanto mais discreta for a solução, maior será as reflexões de onda no material e maior será a largura do band gap. Além disso, no exemplo apresentado, essa diferença de largura do band gap depende do tamanho do raio de projeção $r_{\text {min }}$ utilizado e do coeficiente $q$. Como o valor de $r_{\text {min }}$ adotado nesses exemplos é de apenas 


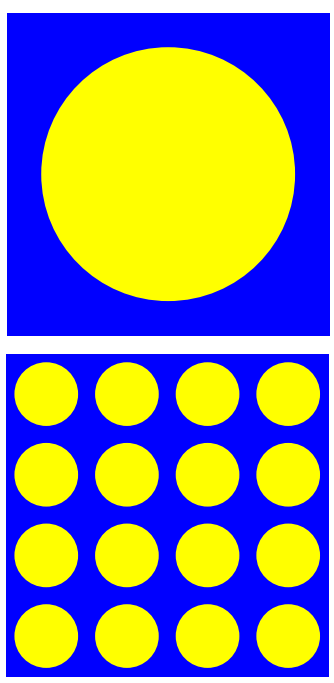

(a)

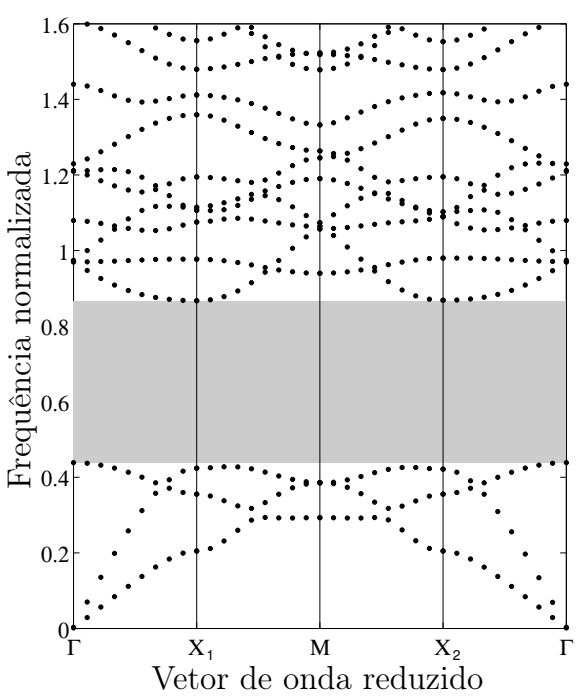

(b)

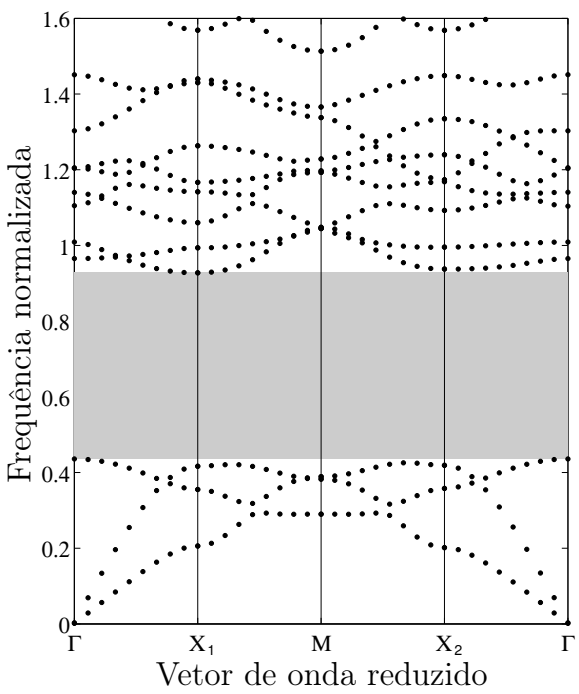

(c)

(a)

Figura 3.24: Influência do efeito piezelétrico na célula unitária com cilindros de PZT-5A inclusos em uma matriz de epóxi: (a) célula unitária e matriz periódica, (b) diagrama de dispersão sem propriedades piezelétricas e (c) com propriedades piezelétricas (amarelo: PZT-5A, azul: epóxi).

5\% do tamanho da célula unitária e $q=8$, a topologia final obtida da otimização já está muito próxima de uma distribuição 0-1 e, portanto, a largura do band gap não se altera significativamente.

O segundo exemplo visa a maximização do band gap entre a $6^{a}$ e ra frequência. Foi obtida numa largura normalizada igual a 0,4512 (ver Fig. 3.26a) e a distribuição de PZT-5A também apresenta a forma de octógono, porém, com regiões de PZT-5A distribuídas em forma de "X". Já o resultado interpretado apresenta um band gap de largura normalizada igual a 0,4578 (ver Fig. 3.26b), com um ganho de 1,6\%. Os próximos resultados, quando interpretados para uma estrutura discreta, não apresentaram ganho superior a 4\%. Portanto, apenas os resultados obtidos da otimização são mostrados.

O terceiro exemplo visa a maximização do band gap entre a "qa e $8^{a}$ frequência. Foi obtida uma largura normalizada igual a 0,0385 (ver Fig. 3.27a). Este intervalo é o menor de todos os obtidos nesse estudo. Nesse caso, há um band gap adicional localizado entre a $3^{a}$ e $4^{a}$ frequência, o qual tem uma largura normalizada igual a 0,2287. Entretanto, este band gap adicional é menor do que o obtido no primeiro caso, isto é, quando o próprio band gap adicional é a própria função objetivo. É possível notar também que PZT-5A concentra-se no meio da célula unitária, com uma geometria mais complexa, e com inclusões de epóxi no próprio PZT-5A.

O quarto exemplo visa a maximização do band gap entre a $10^{a}$ e $11^{a}$ frequência, resultando numa largura normalizada igual a 0,0691 (ver Fig. 3.27b). Neste caso, a célula 

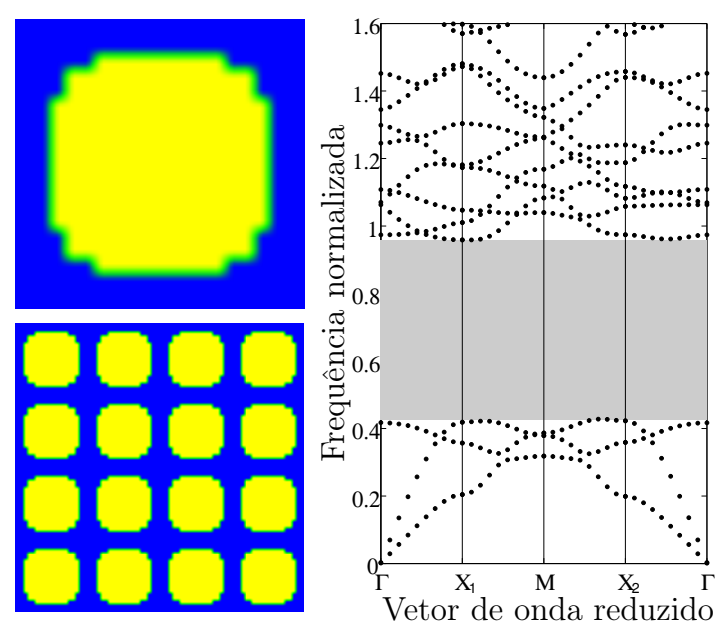

(a)
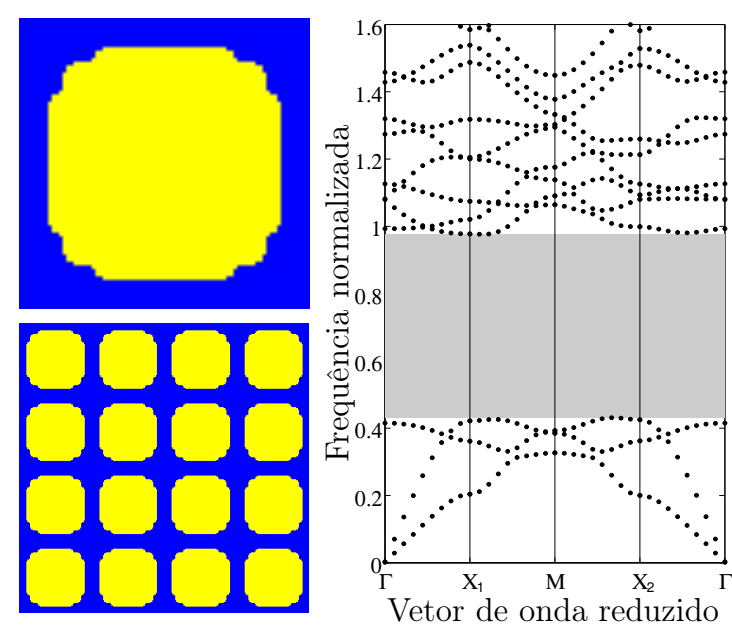

(b)

Figura 3.25: Células unitárias, matrizes periódicas e diagramas de dispersão para a maximização do band gap entre a $3^{a}$ e $4^{a}$ frequência: (a) resultado da otimização topológica e (b) resultado interpretado (amarelo: PZT-5A, azul: epóxi).
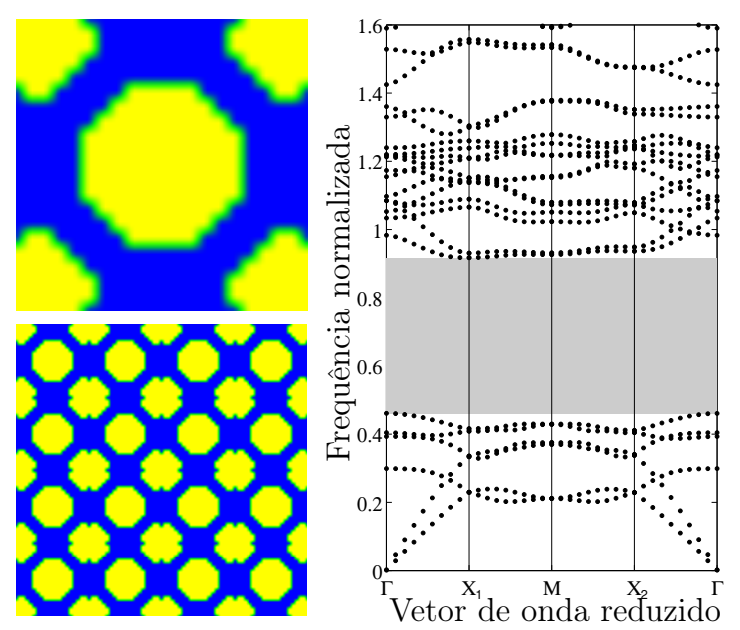

(a)
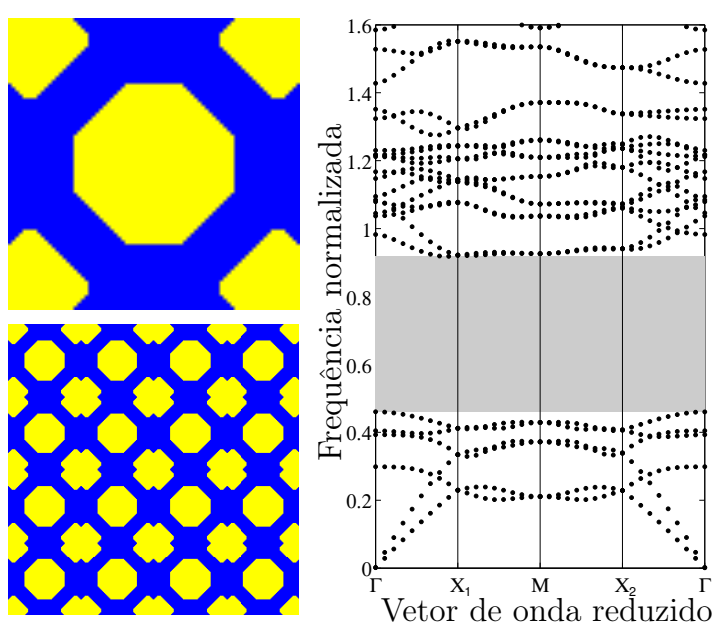

(b)

Figura 3.26: Células unitárias, matrizes periódicas e diagramas de dispersão para a maximização do band gap entre a $6^{a}$ e $7^{a}$ frequência: (a) resultado da otimização topológica e (b) resultado interpretado (amarelo: PZT-5A, azul: epóxi). 

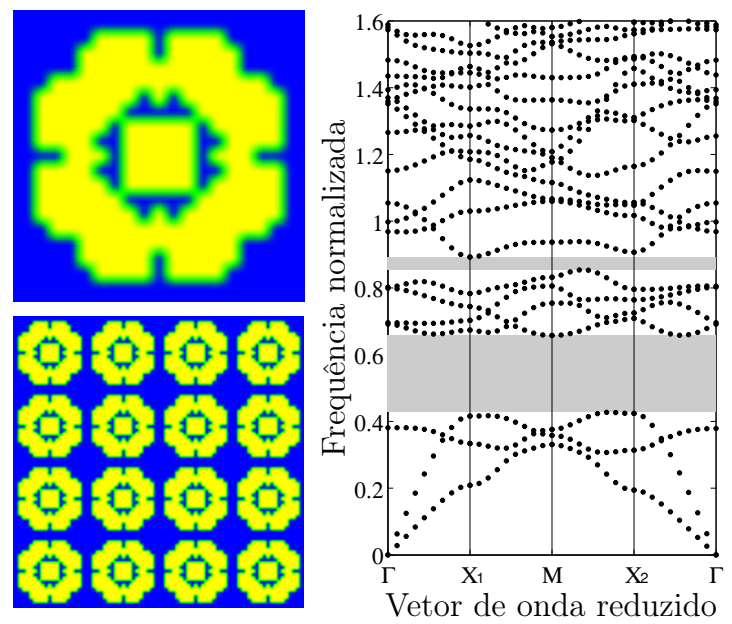

(a)
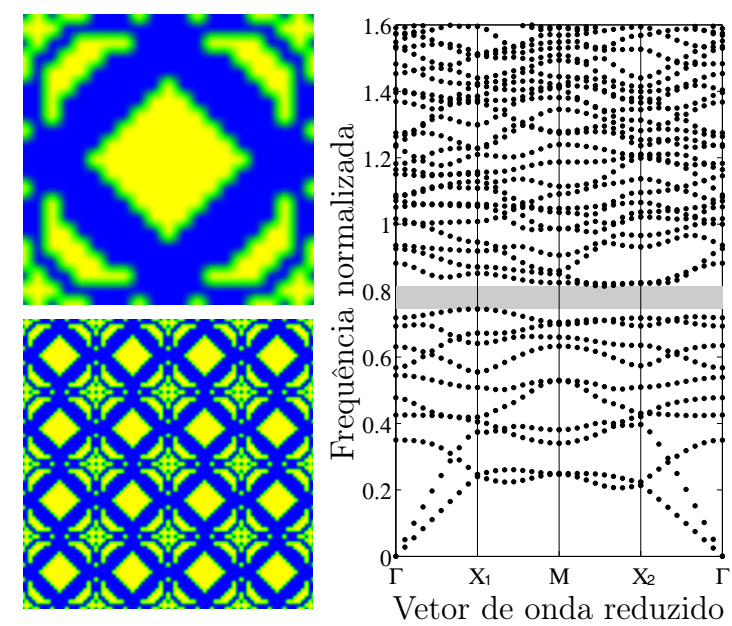

(b)

Figura 3.27: Células unitárias, matrizes periódicas e diagramas de dispersão para a maximização do band gap entre: (a) $7^{a}$ e $8^{a}$ frequência e (b) $10^{a}$ e $11^{a}$ frequência (amarelo: PZT-5A, azul: epóxi).

unitária otimizada apresenta uma região quadrada de PZT-5A na região central, com pequenas regiões de PZT-5A ao redor.

O quinto exemplo visa a maximização do band gap entre a $12^{a}$ e $13^{a}$ frequência. Foi obtida numa largura normalizada igual a 0,5491 (ver Fig. 3.28a) e é a maior obtida nesse estudo. A concentração de PZT-5A com formato de octógono no meio da célula unitária é muito similar (entretanto, não é idêntico) à célula unitária mostrada na Fig. 3.25. Esse exemplo mostra que o diagrama de dispersão é muito sensível em relação ao efeito de escala da topologia.

O sexto exemplo visa a maximização do band gap entre a $13^{a}$ e $14^{a}$ frequência, resultando numa largura normalizada igual a 0,0792 (ver Fig. 3.28b). Nesse caso, há um band gap adicional entre a $17^{a}$ e $18^{a}$ frequência, com largura normalizada igual a 0,1232. Entretanto, esse band gap adicional é menor do que o obtido quando o mesmo é maximizado (ver a seguir).

O sétimo exemplo visa a maximização do band gap entre a $15^{a}$ e $16^{a}$ frequência, resultando numa largura normalizada igual a 0,3266 (ver Fig. 3.29a). A topologia obtida nesse caso é formada por uma região de PZT-5A concentrada no meio da célula unitária, com uma "ilha" de epóxi no seu interior, e partes simétricas de PZT-5A ao redor. $O$ oitavo exemplo visa a maximização do band gap entre a $17^{\text {ra }}$ e $18^{a}$ frequência. Foi obtida uma largura normalizada igual a 0,1584 (ver Fig. 3.29b). Nesse caso, a topologia é quase inversa ao caso anterior, isto é, há uma região pura de PZT-5A no meio da célula unitária e regiões simétricas de PZT-5A com "ilhas" de epóxi. O nono exemplo visa a maximização

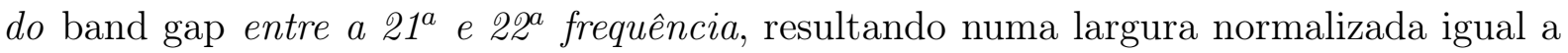
0,2301 (ver Fig. 3.30a) e a topologia é formada por inclusões de PZT-5A na matriz de 

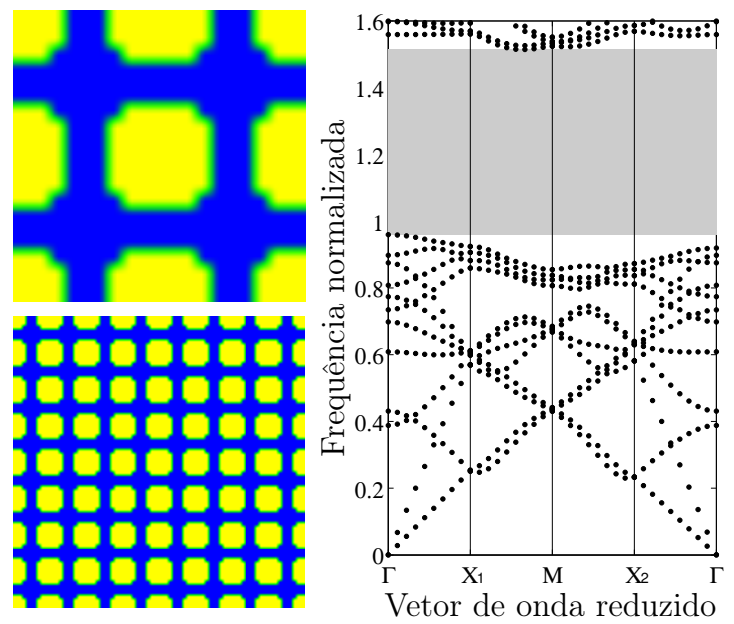

(a)
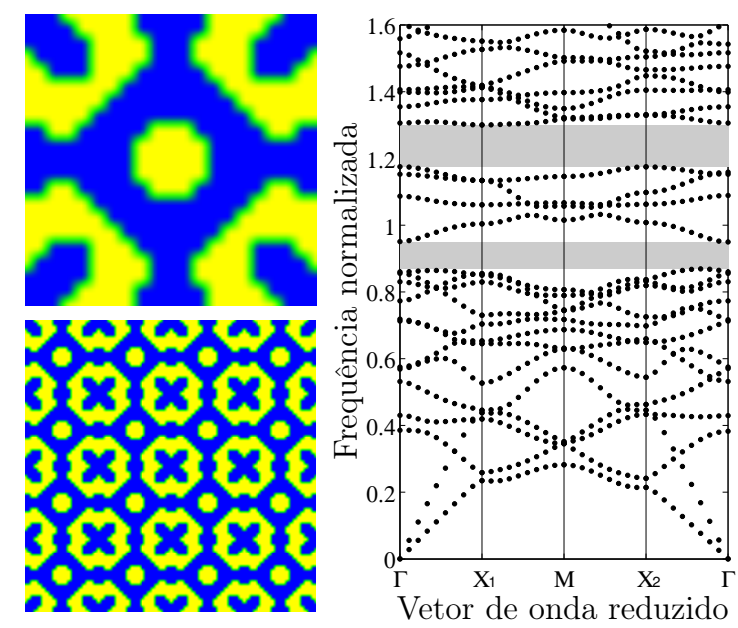

(b)

Figura 3.28: Células unitárias, matrizes periódicas e diagramas de dispersão para a maximização do band gap entre: (a) $12^{a}$ e $13^{a}$ frequência e (b) $13^{a}$ e $14^{a}$ frequência (amarelo: PZT-5A, azul: epóxi).
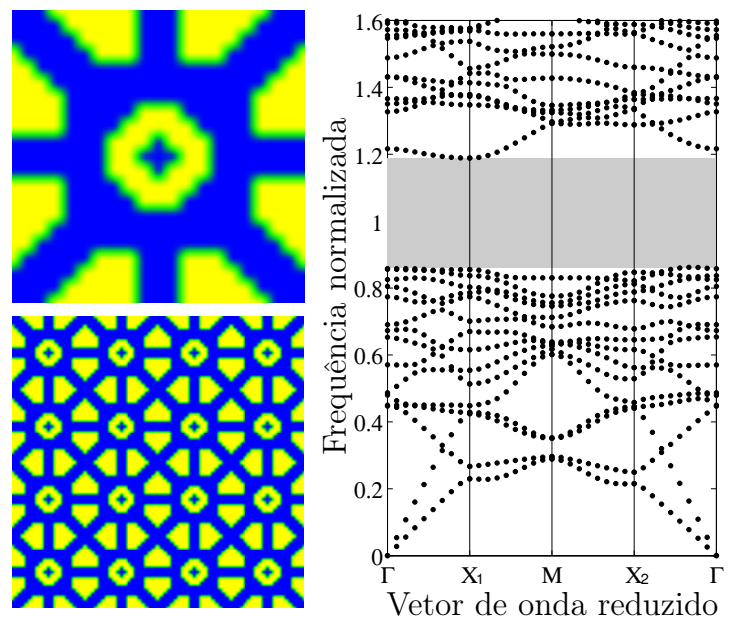

(a)
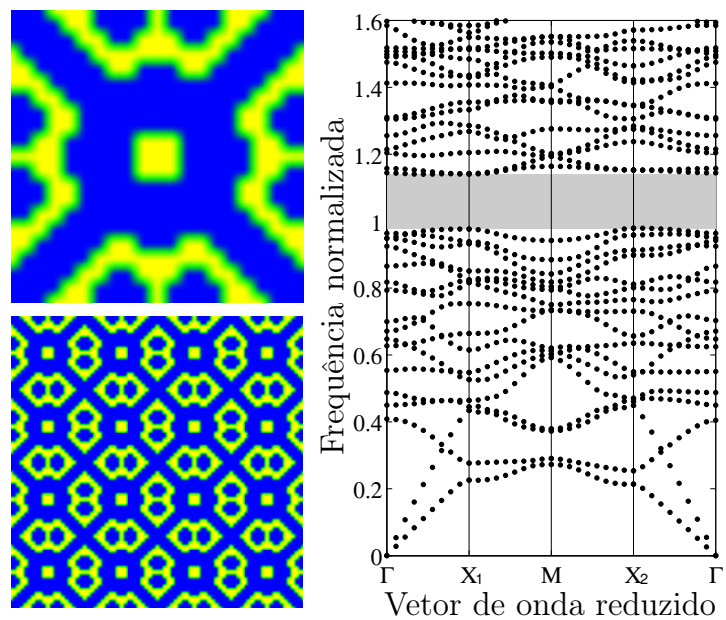

(b)

Figura 3.29: Células unitárias, matrizes periódicas e diagramas de dispersão para a maximização do band gap entre: (a) $15^{a}$ e $16^{a}$ frequência e (b) $17^{a}$ e $18^{a}$ frequência (amarelo: PZT-5A, azul: epóxi).

epóxi. O décimo exemplo visa a maximização do band gap entre a $24^{a}$ e $25^{a}$ frequência, resultando numa largura normalizada igual a 0,3194 (ver Fig. 3.30b).

\section{Influência da gradação de materiais}

Nessa seção, o objetivo é maximizar a largura do primeiro band gap, para diferentes valores do coeficiente de penalização $q$, a fim de avaliar a influência da gradação de material entre os materiais constituintes. O raio de gradação adotado é de $10 \%$ do comprimento da célula unitária. São escolhidos os seguintes valores de $q$ : 0,25, 0,5, 1, 2, 4 e 8. Os valores dos pesos na área de projeção podem ser vistos na Fig. 3.31. Para $q>1$ os pesos da gradação favorecem as propriedades do PZT-5A, enquanto que para valores $q<1$, a projeção favorece as propriedades do epóxi. Para $q=1$, os pesos da gradação variam 

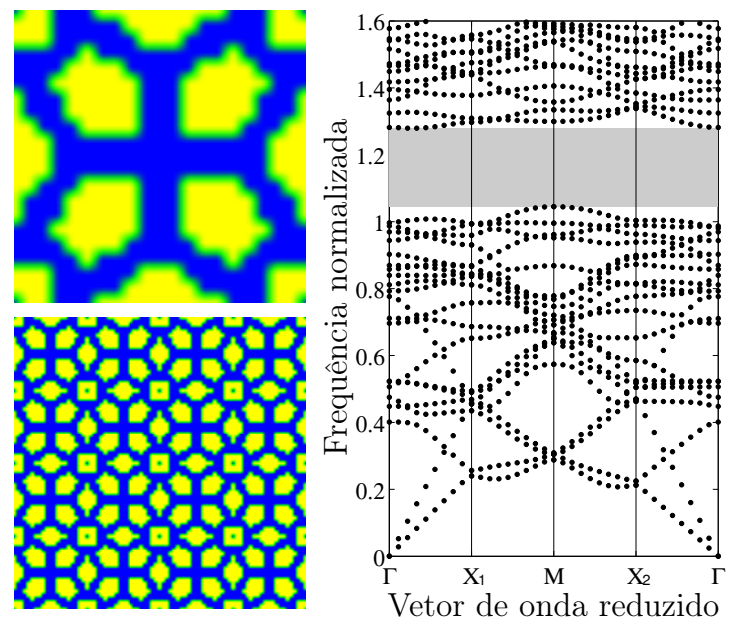

(a)
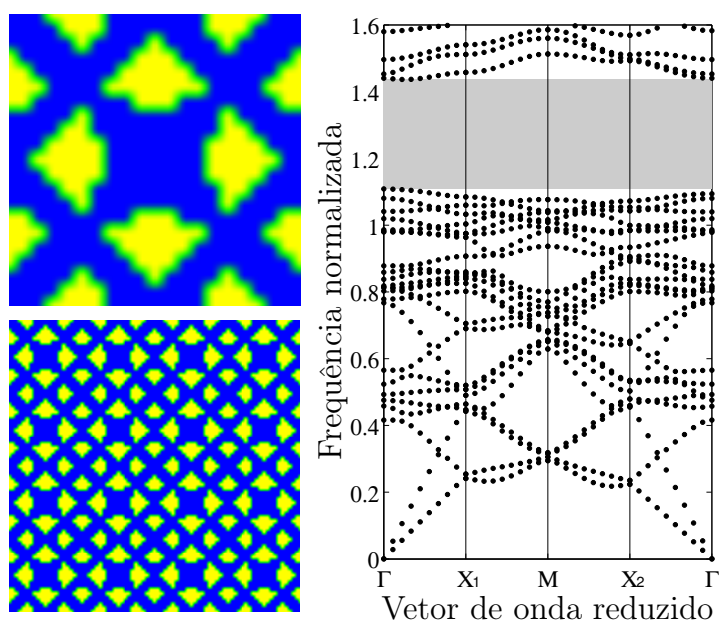

(b)

Figura 3.30: Células unitárias, matrizes periódicas e diagramas de dispersão para a maximização do band gap entre: (a) $21^{a}$ e $22^{a}$ frequência e (b) $24^{a}$ e $25^{a}$ frequência (amarelo: PZT-5A, azul: epóxi).

linearmente em função de $r_{\text {grad }}$.

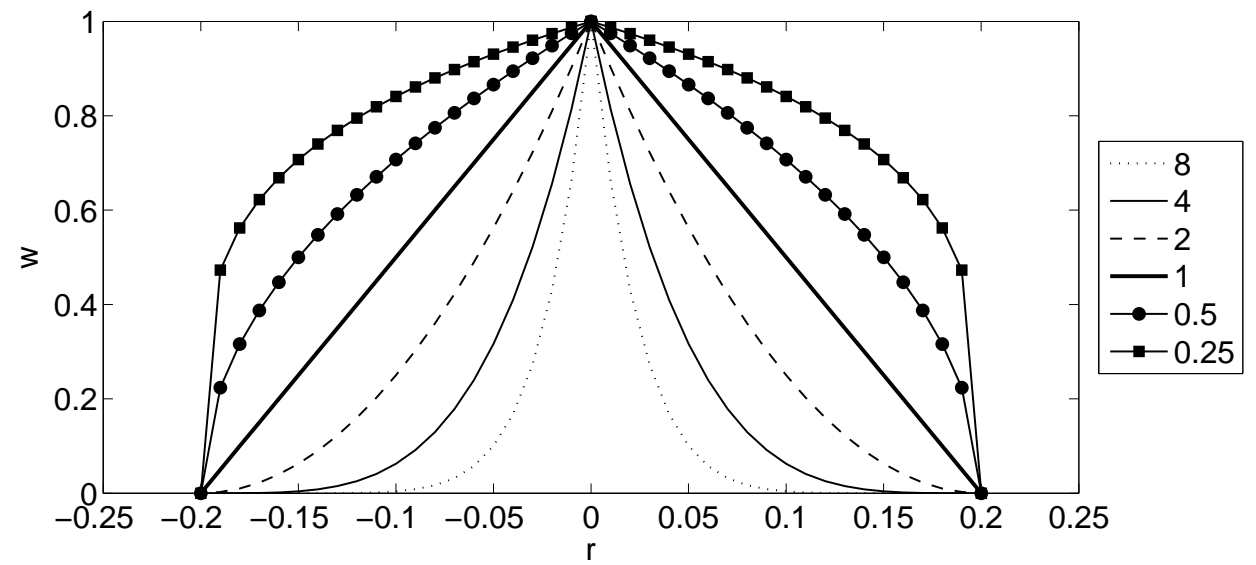

Figura 3.31: Pesos da projeção em função do raio de gradação $r_{\text {grad }}$, para diferentes valores do coeficiente de penalização $q$ (ver Fig. 2.20).

Para cada valor de $q$ o problema de otimização descrito na Seção 3.2 .2 é novamente resolvido. A Fig. 3.32 mostra os resultados para cada valor de $q$. Nota-se que a região de gradação aumenta para os maiores valores de $q$, conforme o esperado. Para valores baixos de $q$, o PZT-5A se apresenta de forma circular e, conforme $q$ aumenta, esta região circular torna-se mais quadrada, de cantos arredondados. Para $q=8$, o PZT-5A assume uma forma semelhante a um octógono.

Primeiramente é escolhido o band gap de baixa ordem entre a $3^{a}$ e $4^{a}$ frequência. As células unitárias otimizadas para os diversos valores $q$ adotados são mostradas na Fig. 3.32. Nota-se que as seis topologias obtidas são semelhantes, embora haja uma mudança gradual desde um formato mais arredondado $(q=0,25)$ até uma forma mais quadrada $(q=8)$. A topologia obtida com $q=8$ assemelha-se à da Fig. 3.25a, a qual também é 
obtida com $q=8$; porém, no caso mostrado na Fig. 3.25a o raio de gradação é menor (5\% do comprimento da célula unitária).

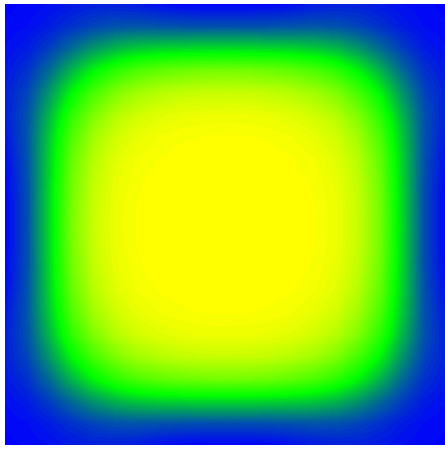

(a) $q=0,25$

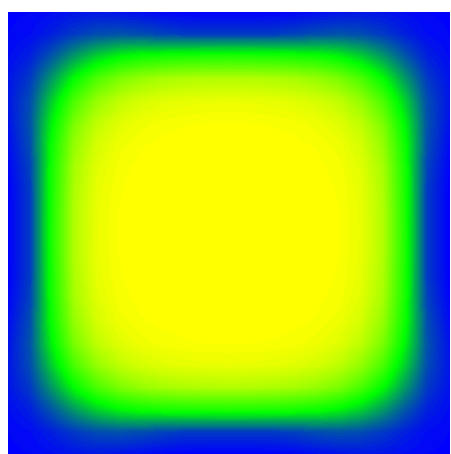

(d) $q=2$

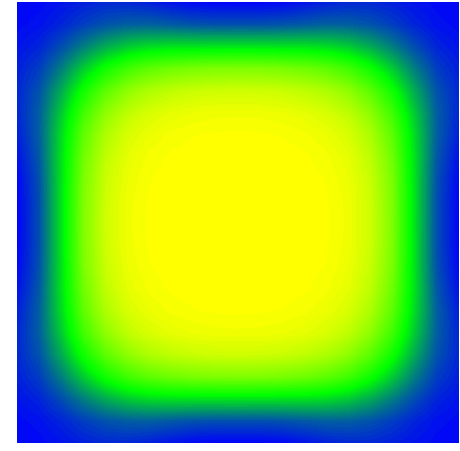

(b) $q=0,50$

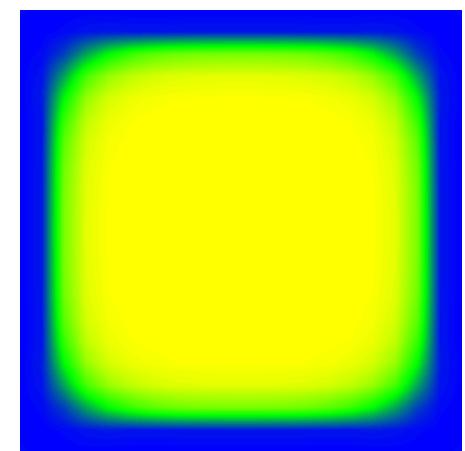

(e) $q=4$

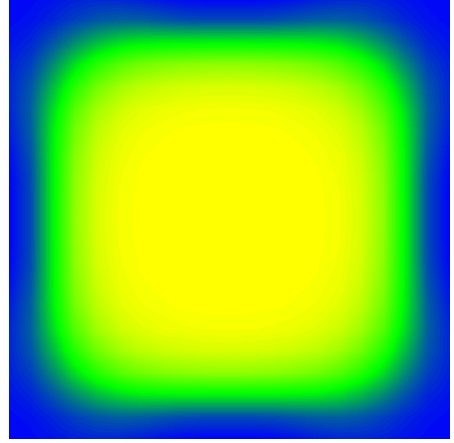

(c) $q=1$

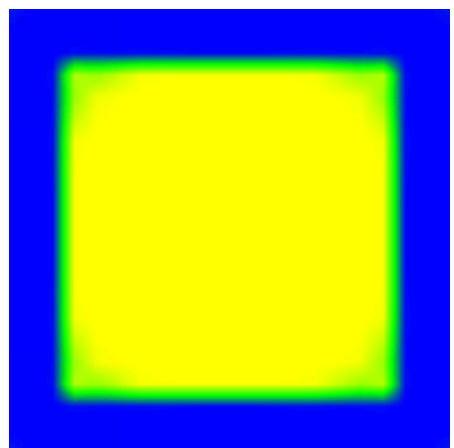

(f) $q=8$

Figura 3.32: Células unitárias que maximizam a largura do band gap entre a $3^{a}$ e $4^{a}$ frequência, para diversos valores do coeficiente de gradação $q$ (amarelo: PZT5A; azul: epóxi).

Os diagramas de dispersão para os 6 valores de $q$ adotados podem ser vistos na Fig. 3.33. Os band gaps são destacados em cinza. A partir destes gráficos é possível notar que a largura do band gap é menor para valores de $q$ iguais a 0,5, 1 e 2. Para $q=0,25$, há um pequeno aumento da largura do band gap em relação à obtida com $q=0,5$. Já para valores maiores de $q$ a largura do band gap aumenta drasticamente, conforme a topologia final torna-se mais discreta. Este fenômeno pode ser observado na Fig. 3.33g. Portanto, o conceito de MGF pode ser utilizado para "sintonizar" os band gaps, satisfazendo assim os requisitos de cada aplicação, mediante o controle do coeficiente $q$. 


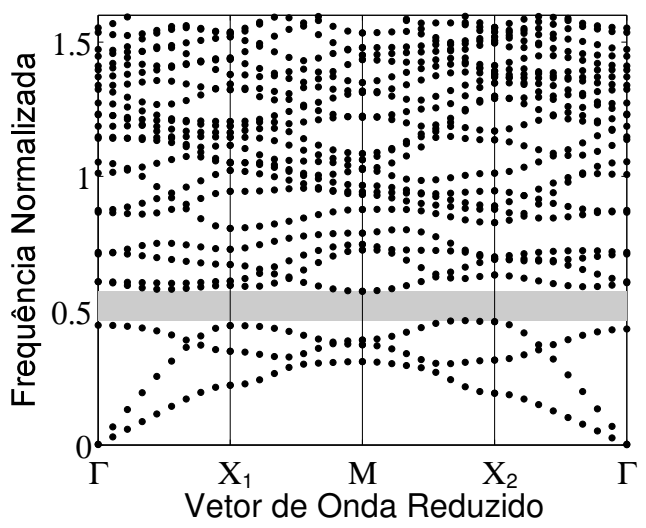

(a) $q=0,25$

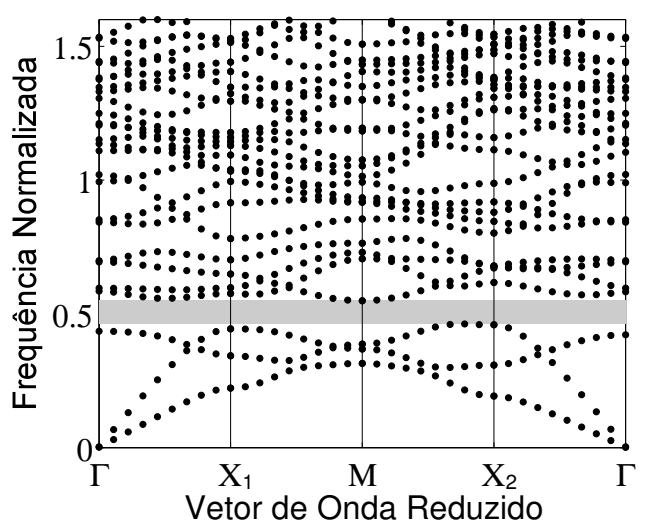

(c) $q=1$

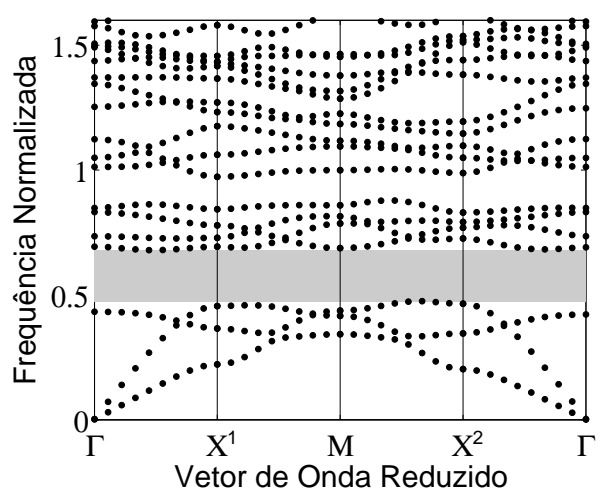

(e) $q=4$

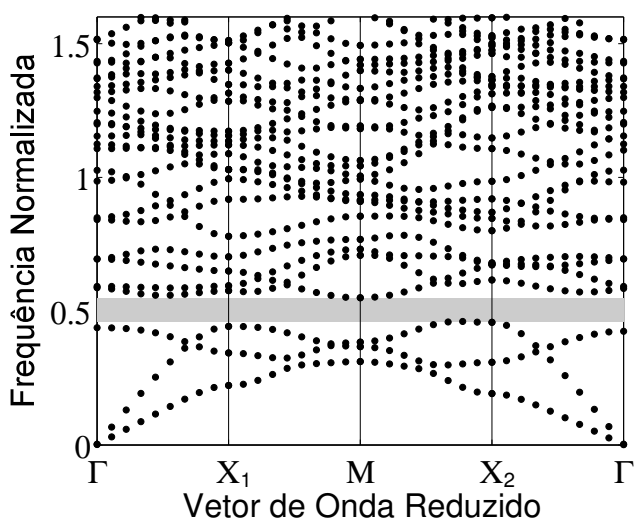

(b) $q=0,50$

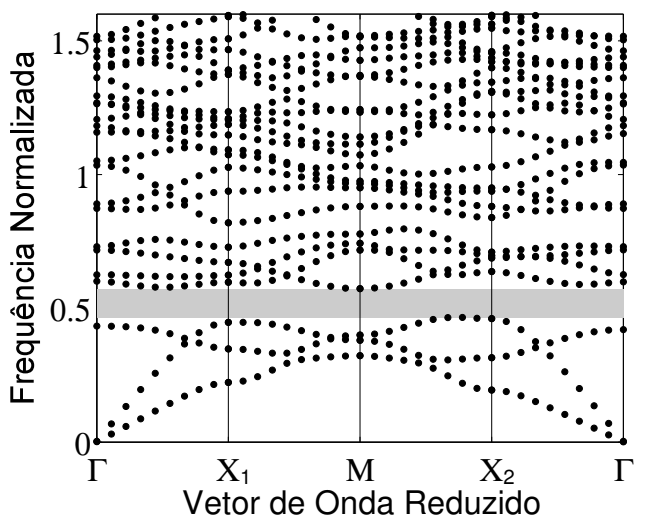

(d) $q=2$

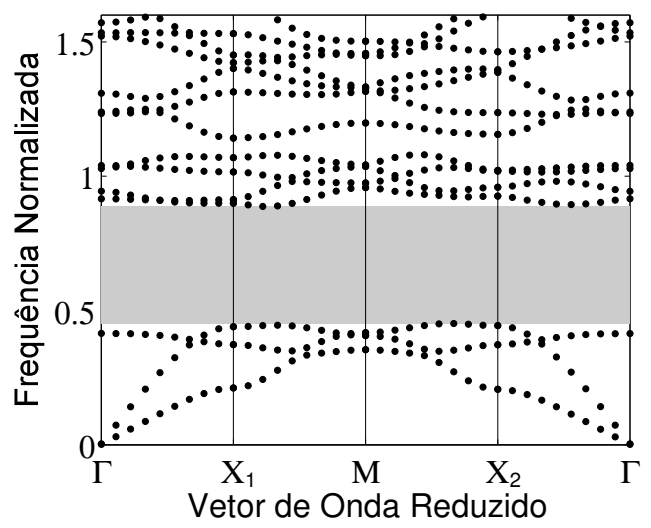

(f) $q=8$

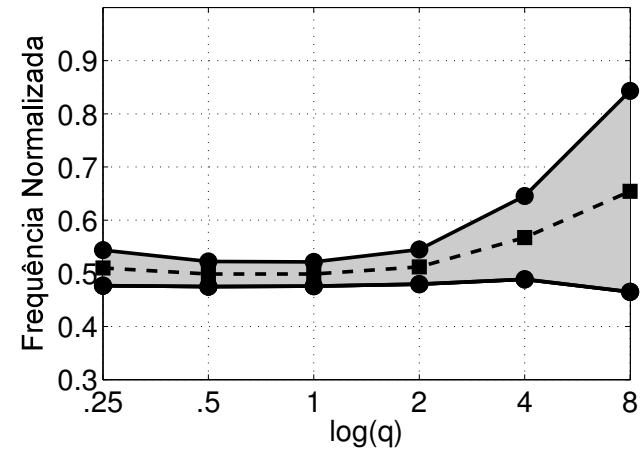

(g)

Figura 3.33: Maximização do band gap entre a $3^{a}$ e $4^{a}$ frequência para diferentes valores de $q$ : (a-f) diagramas de dispersão; (g) Band gaps em função do coeficiente $q$ (as áreas cinzentas representam o band gap). 
Esse mesmo procedimento é realizado visando a maximização do band gap entre a $24^{a}$ e $25^{a}$ frequência, a fim de se observar a influência da gradação de material em band gaps de ordens maiores. As células unitárias otimizadas para os mesmo valores de $q$ adotados são mostradas na Fig. 3.34. Nesse caso também observa-se a uma mudança gradual desde um formato mais arredondado $(q=0,25)$ até uma forma mais retilínea $(q=8)$, com o mesmo número de "ilhas" de PZT-5A na célula unitária.

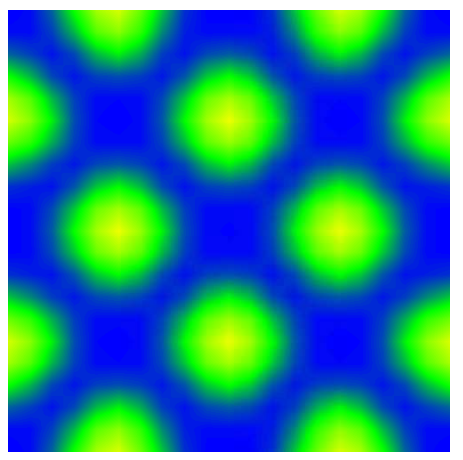

(a) $q=0,25$

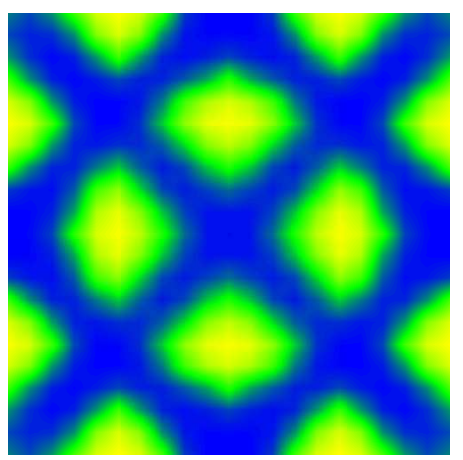

(d) $q=2$

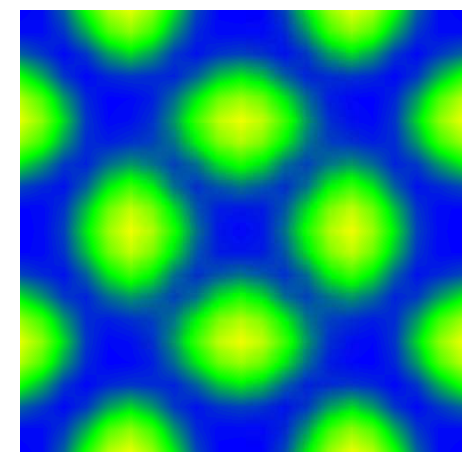

(b) $q=0,50$

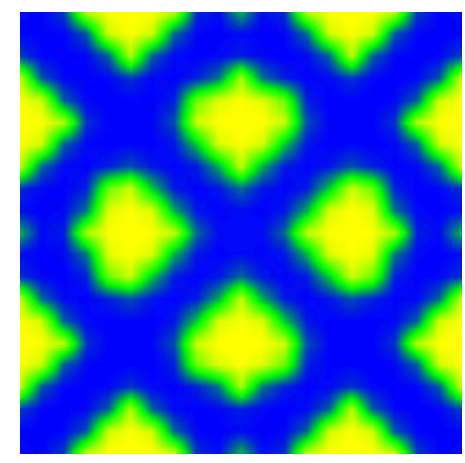

(e) $q=4$

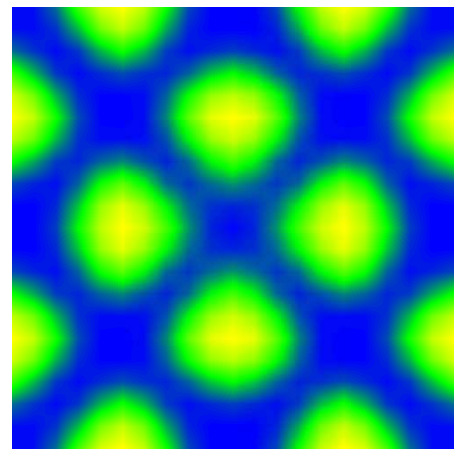

(c) $q=1$

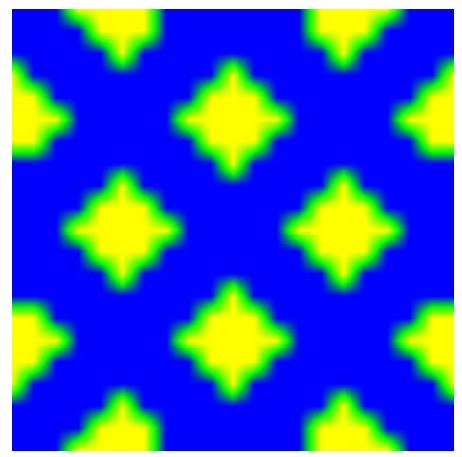

(f) $q=8$

Figura 3.34: Células unitárias que maximizam a largura do band gap entre a $24^{a}$ e $25^{a}$ frequência, para diversos valores do coeficiente de gradação $q$ (amarelo: PZT5A; azul: epóxi).

Os diagramas de dispersão podem ser vistos na Fig. 3.35. Novamente, observa-se que a largura do band gap é menor para valores de $q$ iguais a 0,25 e 0,5 . Para valores maiores de $q$ a largura do band gap também aumenta drasticamente, semelhante ao caso anterior, conforme a topologia final torna-se mais discreta. Este fenômeno pode ser observado na Fig. 3.35g. Portanto, nesse caso o conceito de MGF também pode ser utilizado para "sintonizar" os band gaps mediante o controle do coeficiente $q$. 


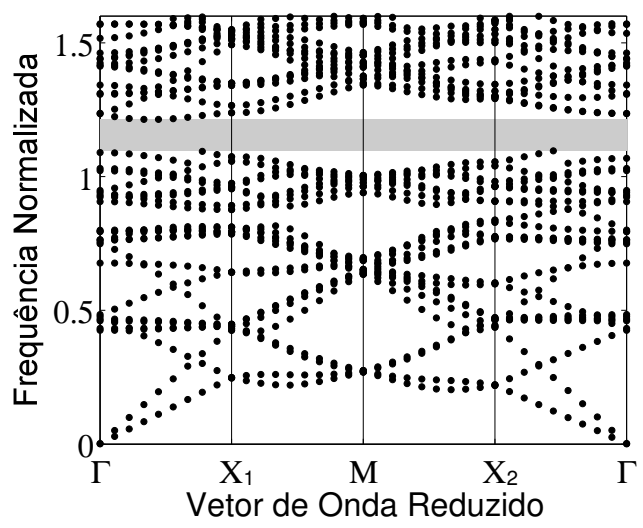

(a) $q=0,25$

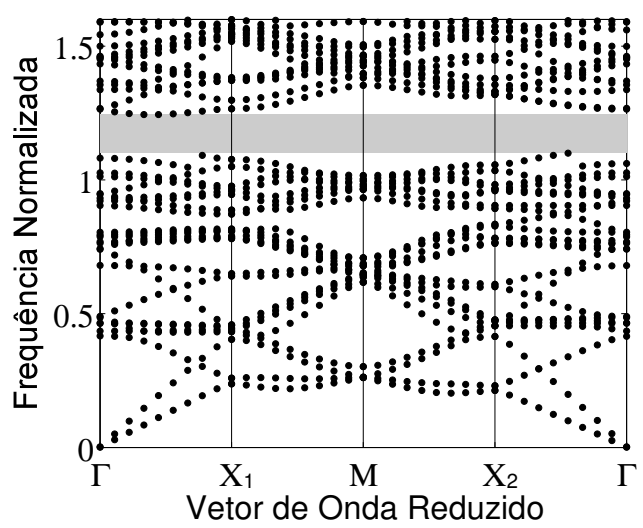

(c) $q=1$

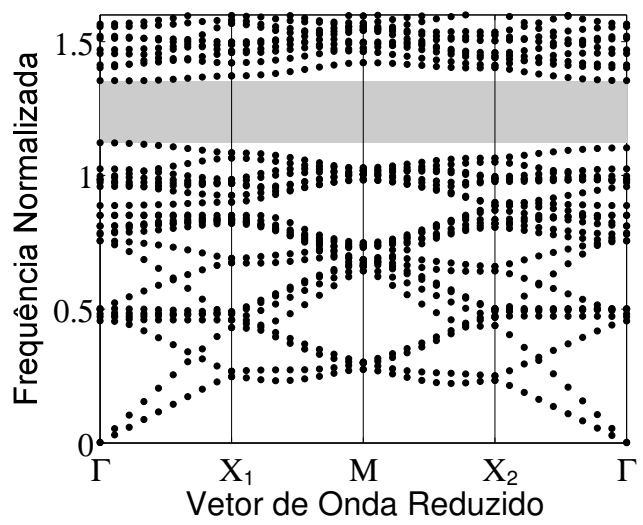

(e) $q=4$

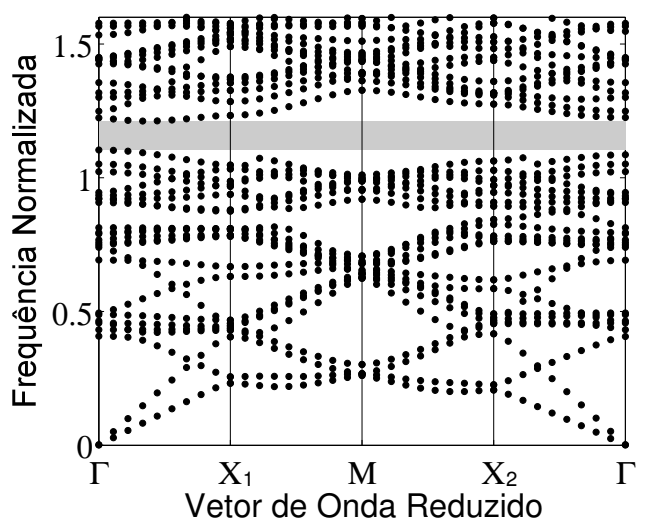

(b) $q=0,50$

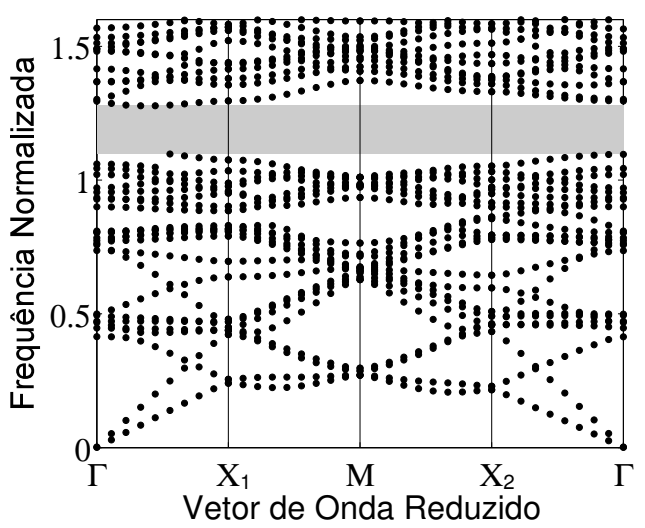

(d) $q=2$

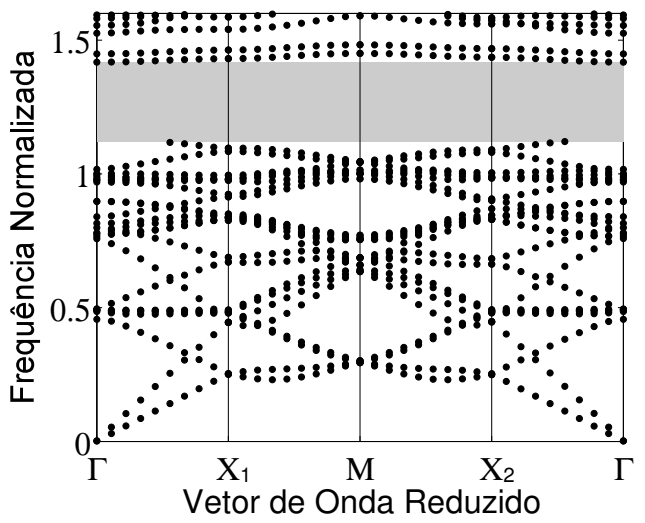

(f) $q=8$

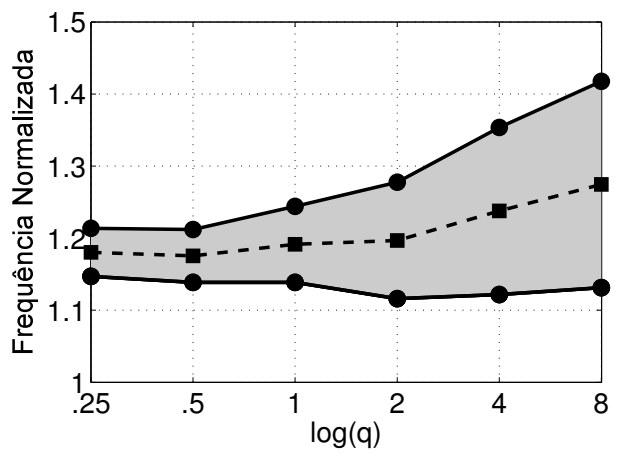

(g)

Figura 3.35: Maximização do band gap entre a $24^{a}$ e $25^{a}$ frequência para diferentes valores de $q$ : (a-f) diagramas de dispersão; (g) Band gaps em função do coeficiente $q$ (as áreas cinzentas representam o band gap). 


\subsection{Considerações Finais}

Neste capítulo é apresentada a viabilidade do projeto de materiais piezocompósitos fonônicos, tanto para posição de band gaps prescrita quanto para maximização de band gaps. São implementados dois modelos numéricos: um unidimensional, baseado no método analítico MMC, que utiliza diversas camadas alternadas de materiais diferentes, e outro bidimensional, que une a teoria de Bloch-Floquet com o MEF e MOT, para projeto de estruturas mais complexas.

Os resultados obtidos a partir do modelo unidimensional mostram que o conceito de MGF pode ser aplicado para projetar materiais piezocompósitos fonônicos com prescrição de largura e posição de band gaps. A maximização de band gaps naturalmente gera topologias de material discretas, entretanto, o conceito de MGF pode ser utilizado para "sintonizar" os band gaps de acordo com os requisitos de cada aplicação.

Utilizando o modelo bidimensional no projeto de MPGF fonônicos, são obtidos diversos materiais piezocompósitos fonônicos visando a maximização de band gaps. Todas as topologias otimizadas obtidas pela metodologia de projeto proposta apresentam inclusões de PZT-5A em uma matriz de epóxi. Nem todos os intervalos entre as frequências de ressonância podem ser abertos, devido a simetria imposta no domínio de projeto. A maximização do primeiro e quinto band gaps apresentam células unitárias semelhantes, porém, as inclusões de PZT-5A do segundo caso são metade do tamanho em relação ao primeiro caso, com diagramas de dispersão distintos. Isso mostra que o efeito de escala da topologia obtida tem grande influência no comportamento de materiais fonônicos. A maximização do terceiro e sexto band gaps apresentam band gaps adicionais no intervalo de frequência analisado. Entretanto, estes band gaps adicionais são menores do que se esses mesmos band gaps são considerados como função objetivo.

A partir da análise da influência de diferentes curvas de gradação de material entre as fases constituintes, no caso do band gap entre a $3^{a}$ e $4^{a}$ frequência, é possível notar que a largura do band gap é menor para valores de $q$ próximos a 1 . Para $q=0,25$, há um pequeno aumento da largura do band gap em relação à obtida com $q=0,5$. Já para valores maiores de $q$ a largura do band gap aumenta drasticamente. Esse efeito é devido a distribuição de material na célula unitária que se torna cada vez mais próxima de uma distribuição discreta para valores altos de $q$. Para o caso do band gap de alta ordem, entre a $24^{a}$ e $25^{a}$ frequência, observa-se também que a largura do band gap é menor para valores baixos de $q(0,25$ e 0,5$)$. Para valores maiores de $q$ a largura do band gap também aumenta drasticamente, conforme a topologia final torna-se mais discreta. É verdade que para valores pequenos de $q$ a distribuição de material tende a ser discreta também; 
entretanto, nos resultados obtidos nesse estudo esse efeito foi só observado para os valores maiores de $q$ adotados. 


\section{$4 \quad$ Projeto de Transdutores Piezocompósitos com Gradação Geométrica}

Neste capítulo é investigada a aplicação do conceito de gradação geométrica (STROMBERG et al., 2011; VATANABE; SILVA, 2011a; VATANABE; PAUliNO; SILVA, 2012), juntamente com o MOT, no projeto de estruturas piezocompósitas do ponto de vista da macroescala, conforme discutido na Seção 1.5. Assim, novos parâmetros de projeto são adicionados, do ponto de vista da gradação geométrica, tais como o número de repetições do padrão no domínio de projeto e a variação das dimensões (ou gradação) do padrão ao longo da estrutura.

Conforme dito anteriormente, uma das propostas desse estudo é expandir o conceito de gradação funcional utilizando o conceito de gradação geométrica, de forma a propor métodos alternativos de projeto de MGF que contornem possíveis dificuldades de manufatura. Além disso, mesmo no MGF, quando observados em uma escala suficientemente pequena, é possível notar que a distribuição de materiais na microestrutura assume uma forma discreta, conforme descrito na Seção 1.5 (ver Fig. $1.5)$.

Nesse estudo investiga-se o conceito expandido de gradação funcional geométrica, baseado em repetições de padrão ao longo do domínio de projeto, porém cada repetição tem um ou mais comprimentos modificados, de forma gradual. Dessa forma, embora a distribuição de materiais seja discreta, suas propriedades (por exemplo, rigidez) alteram-se progressivamente ao longo da estrutura. A repetição de padrões é definida como o número de vezes que uma determinada região pré-definida, denominada padrão, de uma estrutura qualquer é repetida ao longo de uma ou mais direções. A gradação geométrica consiste em alterar continuamente (ou gradativamente) uma ou mais dimensões do padrão a cada repetição ao longo da estrutura (ver Fig. 4.1).

Enquanto que o estudo apresentado no Capítulo 2 limita-se a aplicações quasi-estáticas de microestruturas piezocompósitas na coleta de energia segundo uma abordagem de 


\section{Gradação Funcional de Material}

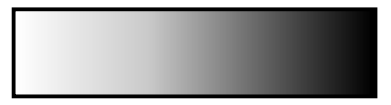

célula unitária domínio de projeto
Gradação Funcional Geométrica domínio de projeto

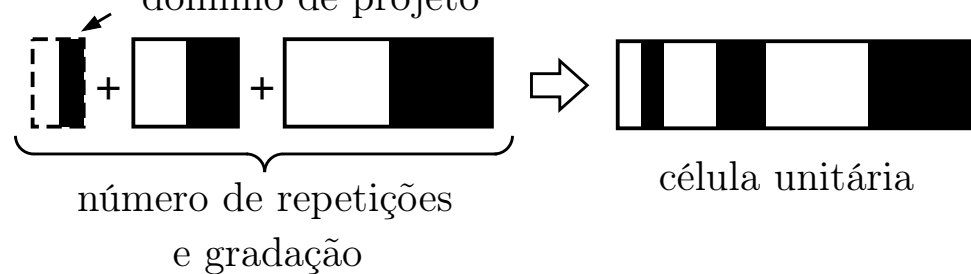

Figura 4.1: Substituição da gradação de material pela gradação geométrica, no interior da célula unitária.

microescala, nesse tópico é investigado a aplicação do conceito de gradação geométrica (STROMBERG et al., 2011) no projeto de estruturas piezocompósitas considerando a mesma aplicação, porém em aplicações dinâmicas, segundo uma abordagem de macroescala. A topologia da estrutura piezocompósita é obtida distribuindo-se dois materiais no domínio de projeto. O modelo de material adotado assume que as propriedades em cada ponto do domínio da célula pode variar de uma fase a outra proporcionalmente a um parâmetro $\rho$, que é a variável de projeto do problema de otimização. Uma técnica de controle de gradiente do material é implementada baseada na projeção de Bruns e Tortorelli (2001).

Este capítulo é organizado da seguinte forma: na Seção 4.1 é brevemente apresentada a formulação do MEF para modelagem completa do sistema em estudo. Na Seção 4.2 é apresentada a formulação do problema de otimização. Na Seção 4.3 é apresentada a implementação numérica do conceito de gradação geométrica, juntamente com os diferentes domínios de projeto adotados neste estudo. Na Seção 4.4 são apresentados os resultados numéricos mostrando a influência do conceito de gradação geométrica no projeto de dispositivos de coleta de energia. Finalmente, na Seção 4.5 são discutidas algumas considerações finais do estudo apresentado neste capítulo.

\subsection{Método de Elementos Finitos Dinâmico para Materiais Piezelétricos}

Assumindo que os deslocamentos e potencial elétrico tenham comportamento harmônico $\left(\mathbf{u}=\mathbf{u}_{e} e^{i \omega t}, \phi=\phi_{e} e^{i \omega t}\right)$ e frequência de excitação $\omega$, o sistema piezelétrico dinâmico com amortecimento estrutural pode ser escrito na forma de elementos finitos da seguinte forma: 


$$
\begin{aligned}
& -\omega^{2}\left[\begin{array}{cc}
\mathbf{M} & \mathbf{0} \\
\mathbf{0} & \mathbf{0}
\end{array}\right]\left\{\begin{array}{l}
\mathbf{U} \\
\mathbf{\Phi}
\end{array}\right\}+i \omega\left(\left[\begin{array}{ll}
\mathbf{C} & \mathbf{0} \\
\mathbf{0} & \mathbf{0}
\end{array}\right]+\left[\begin{array}{cc}
\mathbf{0} & \mathbf{0} \\
\mathbf{0} & \mathbf{K}_{R}
\end{array}\right]\right)\left\{\begin{array}{l}
\mathbf{U} \\
\mathbf{\Phi}
\end{array}\right\}+ \\
& +\left[\begin{array}{ll}
\mathbf{K}_{\mathbf{u u}} & \mathbf{K}_{\mathbf{u} \phi} \\
\mathbf{K}_{\mathbf{u} \phi}^{t} & \mathbf{K}_{\phi \phi}
\end{array}\right]\left\{\begin{array}{l}
\mathbf{U} \\
\mathbf{\Phi}
\end{array}\right\}=\left\{\begin{array}{l}
\mathbf{F} \\
\mathbf{Q}
\end{array}\right\}
\end{aligned}
$$

onde $\omega$ é a frequência angular, $\mathbf{M}, \mathbf{C}, \mathbf{K}_{R}, \mathbf{K}_{\mathbf{u u}}, \mathbf{K}_{\mathbf{u} \phi}$ e $\mathbf{K}_{\phi \phi}$ são as matrizes de massa, de amortecimento, do resistor, de rigidez, piezelétrica e dielétrica, respectivamente, e $\mathbf{U}, \mathbf{\Phi}, \mathbf{F}$ e $\mathbf{Q}$ são os vetores de deslocamento, potencial elétrico, de força e carga elétrica, respectivamente. O MATLAB ${ }^{\circledR}$ possui uma biblioteca capaz de calcular sistemas complexos.

A matriz de amortecimento estrutural adotada é dada por:

$$
\mathbf{C}=\alpha \mathbf{K}_{\mathbf{u u}}
$$

onde $\alpha$ é o fator de amortecimento. A matriz do resistor, em uma análise harmônica, é obtida da seguinte forma:

$$
\mathbf{K}_{R}=\left(-\frac{1}{\omega^{2} R}\right)\left[\begin{array}{cc}
1 & -1 \\
-1 & 1
\end{array}\right]\left\{\begin{array}{l}
\phi_{1} \\
\phi_{2}
\end{array}\right\}
$$

onde $\phi_{1}$ e $\phi_{2}$ são os graus de liberdade elétricos nos quais o resistor está conectado. Como os eletrodos acoplam os nós das faces inferior e superior, essa matriz do resistor é adicionada apenas em um dos nós de cada eletrodo.

O coeficiente de acoplamento eletromecânico $k$ e a potência elétrica no resistor são calculados da seguinte forma (LERCH, 1990):

$$
k^{2}=\frac{(\text { Energia Eletromecânica })^{2}}{(\text { Energia Elétrica)(Energia Mecânica) }}=\frac{\left(\mathbf{U}^{t} \mathbf{K}_{\mathbf{u} \phi} \boldsymbol{\Phi}\right)^{2}}{\left(\mathbf{U}^{t} \mathbf{K}_{\mathbf{u u}} \mathbf{U}\right)\left(\Phi^{t} \mathbf{K}_{\phi \phi} \boldsymbol{\Phi}\right)}
$$

$\mathrm{e}$

$$
P=\frac{V_{r m s}^{2}}{R}
$$

onde $V_{r m s}$ é a voltagem média quadrática, ou eficaz, obtida nos eletrodos e R é o valor do resistor acoplado ao transdutor. 


\subsection{Formulação do Problema de Otimização Topológica}

O projeto de transdutores piezelétricos para coleta de energia pode ser utilizado em aplicações quasi-estáticas, onde a frequência de excitação é muito menor do que a frequência de ressonância, conforme mostrado no Capítulo 2, ou para aplicações dinâmicas, onde o objetivo é trabalhar com o transdutor operando em uma faixa de frequência o mais próxima possível de alguma frequência de ressonância. Em aplicações quasi-estáticas, normalmente o objetivo é maximizar a conversão de energia mecânica em elétrica, a qual pode ser medida através de diversos critérios, sendo o coeficiente de acoplamento eletromecânico $(k)$ o mais comumente utilizado. Já no caso de aplicações dinâmicas, geralmente investiga-se a maximização direta da potência elétrica gerada em uma determinada carga (RUPP et al., 2009; LEE; YOUN, 2011; EL-SABBAGH; BAZ, 2011). Ao se trabalhar com o coeficiente $k$ não é necessário incluir no projeto do transdutor a carga onde será utilizada ou armazenada a energia elétrica convertida, tais como resistores ou capacitores, por exemplo. Já em aplicações dinâmicas há a necessidade de incluir a carga no modelo, o que adiciona mais variáveis no projeto, tais como a localização e dimensões dos eletrodos, o valor ótimo da carga, a necessidade de considerar amortecimento estrutural para evitar problemas numéricos quando se opera na frequência de ressonância, tornando assim o projeto de transdutores piezelétricos mais complexo. Entretanto, aspectos importantes no projeto desses transdutores são muitas vezes negligenciados (VATANABE; SILVA, 2012).

Recentemente, técnicas de otimização tem sido utilizadas para projeto de coletores de energia. Assim, Zheng et al. (ZHENG; CHANG; GEA, 2009) utilizam otimização topológica para maximizar a conversão de energia mecânica em elétrica para aplicações estáticas de placas piezelétrica. Elka e Bucher (ELKA; BUCHER, 2009) obtém a distribuição otimizada dos eletrodos a fim de se obter filtros de modos de vibrar de atuadores piezelétricos. Já Rupp et al. (RUPP et al., 2009) utilizaram uma abordagem computacional para projetar um sistema dinâmico de coleta de energia composto por camadas de piezocerâmicas acopladas a um substrato. As variáveis de projeto foram a distribuição de material piezelétrico no substrato e o sentido de polarização, de forma a maximizar a potência elétrica gerada em um resistor. Chen et al. (CHEN et al., 2010) propuseram um método de otimização topológica utilizando level-sets para projetar dispositivos de coleta de energia. Nakasone e Silva (NAKASONE; SILVA, 2010) apresentaram uma formulação para projetar placas laminadas piezelétricas utilizadas em sensores piezelétricos, atuadores e em coleta de energia. Lee e Youn (LEE; YOUN, 2011) apresentaram um método de segmentação de uma camada piezelétrica acoplada a uma superfície vibratória, orientado através de linhas de inflexão de múltiplos modos de vibrar, de modo a minimizar o cancelamento de voltagem. 
El-Sabbagh e Baz (EL-SABBAGH; BAZ, 2011) investigaram o emprego de múltiplos pares de eletrodos para evitar o problema de cancelamento de voltagem em atuadores piezelétricos bilaminares aplicados a sistemas de coleta de energia.

Na abordagem em estudo, a otimização consiste em se distribuir o material no interior de um domínio de forma a extremizar uma função objetivo desejada (rigidez, deslocamento gerado, etc.). As etapas do MOT no projeto de transdutores piezelétricos são apresentadas na Fig. 4.2. Definido o domínio de projeto, onde são aplicadas as condições de contorno e o carregamento, o mesmo é então discretizado em elementos finitos. Então, definem-se as repetições do padrão e a gradação geométrica ao longo do domínio, assim como a região onde serão dispostas as variáveis de projeto. Assim, é realizado o processo iterativo de otimização, até que seja obtida a distribuição de material otimizada. Finalmente, é realizada a etapa de pós-processamento, onde esse resultado é interpretado, verificado e encaminhado para fabricação.

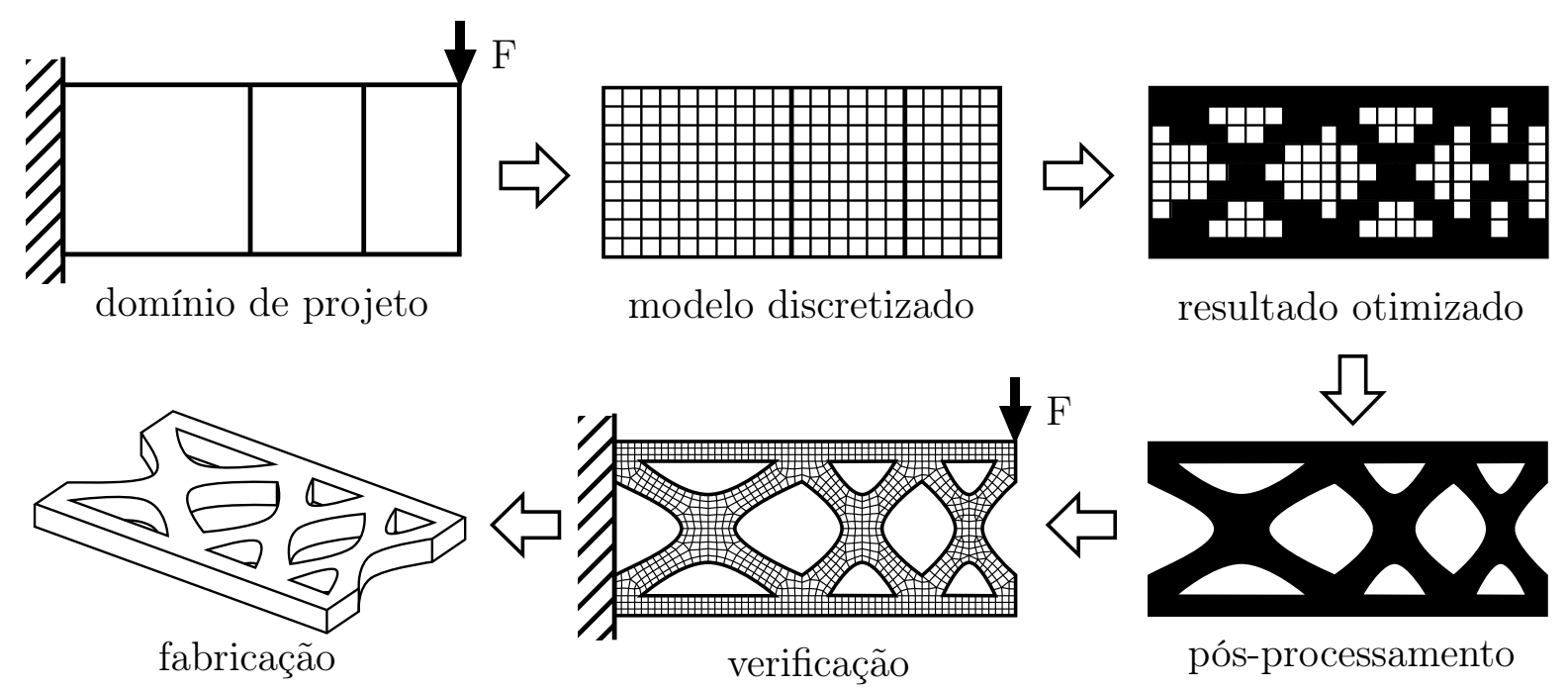

Figura 4.2: Etapas do projeto de transdutores piezelétricos utilizando o MOT.

Para a aplicação de coleta de energia adotada como exemplo nesse estudo, utiliza-se um domínio retangular formado por duas camadas piezelétricas, o qual é engastado em uma das extremidades, sendo aplicado uma força harmônica na extremidade livre. As superfícies são conectadas por eletrodos, onde também é acoplado um resistor, que faz o papel da carga a ser alimentada eletricamente. O objetivo nesse estudo é a maximização da potência elétrica gerada na carga.

Assim, a formulação do problema discreto é definida como: 


$$
\begin{array}{ll}
\underset{\mathbf{d}}{\operatorname{Maximizar}:} & P \\
\text { Restrito a: } & \text { Equação do sistema dinâmico (B.18) } \\
& 0 \leq \mathbf{d} \leq 1 \\
& V(\mathbf{d})=\sum_{i=1}^{N} \mathbf{d} d \Omega \leq V_{s}
\end{array}
$$

onde $P$ é a potência elétrica gerada no resistor, a qual é calculada através da diferença de potencial gerada nos eletrodos (ver Eq. (4.5)). O vetor $\mathbf{d}$ representa as pseudo-densidades sobrepostas às variáveis de projeto $\rho_{N}$, conforme descrito na Seção 2.3.5.

\subsubsection{Modelo de Material}

Nesse estudo a formulação do problema de otimização topológica utiliza um modelo de material baseado no SIMP (Solid Isotropic Material With Penalization) (BENDSøE; SIGMUND, 2003), o qual define as propriedades efetivas localmente, isto é, em cada elemento finito, da seguinte forma:

$$
\begin{aligned}
\mathbf{c}^{h} & =\rho^{p_{c}} \mathbf{c}_{\text {mat }_{A}}+\left(1-\rho^{p_{c}}\right) \mathbf{c}_{\text {mat }_{B}} \\
\mathbf{e}^{h} & =\rho^{p_{e}} \mathbf{e}_{\text {mat }_{A}}+\left(1-\rho^{p_{e}}\right) \mathbf{e}_{\text {mat }_{B}} \\
\boldsymbol{\varepsilon}^{h} & =\rho^{p_{\varepsilon}} \boldsymbol{\varepsilon}_{\text {mat }_{A}}+\left(1-\rho^{p_{\varepsilon}}\right) \boldsymbol{\varepsilon}_{\text {mat }_{B}}
\end{aligned}
$$

onde $\mathbf{c}_{\text {mat }_{i}}$, $\mathbf{e}_{\text {mat }_{i}}$ e $\varepsilon_{\text {mat }_{i}}$ correspondem aos tensores elástico, piezelétrico e dielétrico, respectivamente, dos materiais A e B. A variável $\rho$ é a pseudo-densidade que descreve a quantidade de material em cada ponto do domínio, a qual pode assumir valores entre 0 e 1 , e $p_{c}, p_{e}$ e $p_{\varepsilon}$ são os coeficientes de penalização. Esses coeficientes de penalização são definidos de forma a satisfazer as duas condições descritas por Kim et al. (KIM et al., 2010) (ver Seção 2.2.1).

Conforme descrito na Seção 2.2.1, uma solução topológica pode ser obtida aplicando coeficientes de penalização $p_{c}, p_{e}$ e $p_{\varepsilon}$ no modelo de material para recuperar a distribuição discreta 0-1, juntamente com técnicas de controle de gradiente na distribuição de material, tais como filtros ou técnicas de projeção (BENDSøE; SIGMUND, 2003).

\subsection{Implementação Numérica}

A implementação do conceito de gradação geométrica é baseada no conceito do MTOP - Multiresolution Topology Optimization (NGUYEN et al., 2010), onde as variáveis de projeto são desvinculadas na malha de elementos finitos (ver Fig. 4.3). Para isso, 
adiciona-se uma camada de pseudo-densidades, as quais são funções das variáveis de projeto segundo um método de projeção similar ao apresentado na Seção 2.3.5.

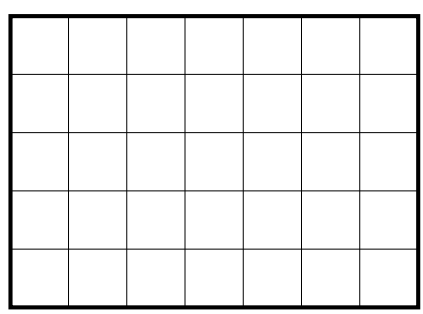

(a)

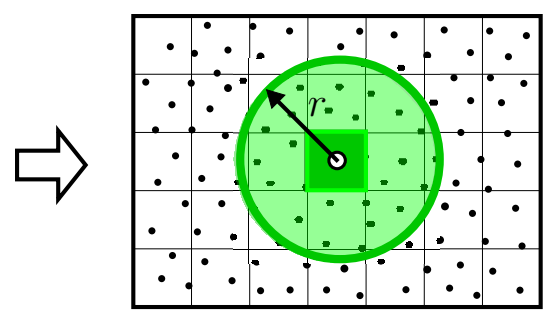

(b)

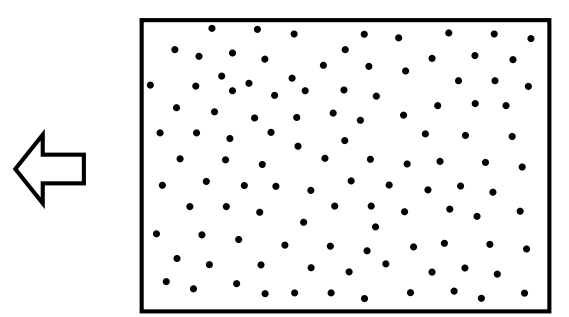

(c)

Figura 4.3: Ilustração do conceito MTOP: (a) malha de elementos finitos; (b) malha de pseudo-densidades e (c) variáveis de projeto.

A repetição de padrões é definida como o número de vezes que uma determinada região pré-definida, denominada padrão, de uma estrutura qualquer é repetida ao longo de uma ou mais direções. A gradação geométrica consiste em alterar (esticar ou encolher) uma ou mais dimensões do padrão a cada repetição ao longo da estrutura (STROMBERG et al., 2011).

Definida a gradação dos padrões, distribui-se as variáveis de projeto nos nós da maior região, as quais são consideradas como variáveis de projeto primárias, mostradas em cinza na Fig. 4.4. Estas variáveis são mapeadas nas regiões repetidas, criando as variáveis de projeto mapeadas. mostradas em preto na Fig. 4.4. Nesta figura também é mostrado a repetição de padrão através do gradiente de cores. Realizado o mapeamento das variáveis, calculam-se as pseudo-densidades de cada elemento no domínio de projeto inteiro.

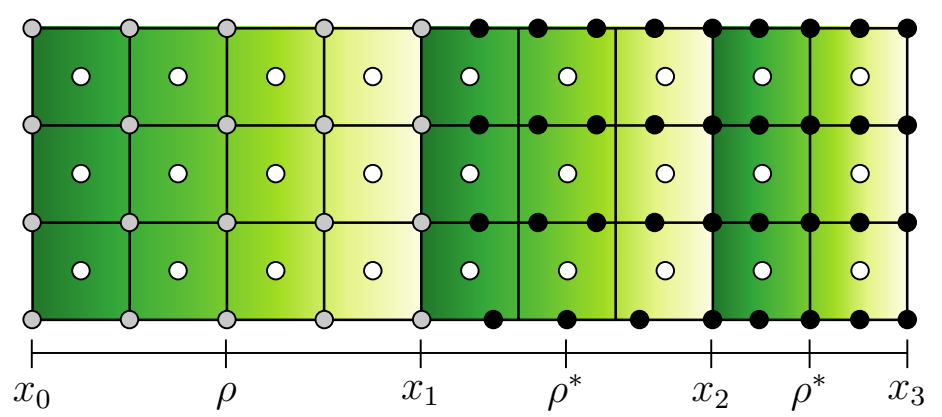

Figura 4.4: Exemplo de gradação geométrica em uma direção; as variáveis de projeto nodais primárias são indicadas em cinza, as variáveis de projeto mapeadas em preto e as repetições de padrão são indicadas pelo gradiente de cores.

Para a gradação geométrica em uma direção, o padrão é definido pela região do domínio de projeto limitada por duas coordenadas, denominadas $x_{n-1}$ e $x_{n}$, para o $n$-ésimo padrão. Na Fig. 4.4, por exemplo, a gradação geométrica é dada na direção $x$, e o padrão 1 é definido pelas coordenadas $x_{0}$ e $x_{1}$.

As variáveis de projeto mapeadas são definidas da seguinte forma: 


$$
x^{*}=\sum_{i=0}^{n-1}\left(x_{i+1}-x_{i}\right)+\alpha_{n}\left(x-x_{n}\right)
$$

onde $\alpha$ é um fator de escala do $n$-ésimo padrão em relação ao maior padrão (que possui as variáveis de projeto primárias). Este fator de escala é definido por:

$$
\alpha_{n}=\frac{x_{n+1}-x_{n}}{x_{1}-x_{0}}
$$

As variáveis de projeto mapeadas tem o mesmo valor dos respectivos valores das variáveis de projeto primárias. O solver adotado na otimização topológica modifica os valores das variáveis de projeto primárias e, indiretamente, as variáveis de projeto mapeadas são atualizadas.

Os fatores de peso da função de projeção devem ser reformulados para a gradação geométrica. Desta forma, a região de projeção deixa de ser circular de raio constante e assume uma forma elíptica, em função da posição de cada elemento. Assim, a maior dimensão da elipse mede $2 r_{\text {min }}$ e as distâncias entre os nós são multiplicadas pelo fator de escala $\alpha$ (ver Fig. 4.5c). Nas regiões próximas às fronteiras dos padrões, a região de projeção assume uma forma híbrida de círculo e elipse (ver Fig. 4.5b). Os pesos da projeção são calculados da seguinte forma:

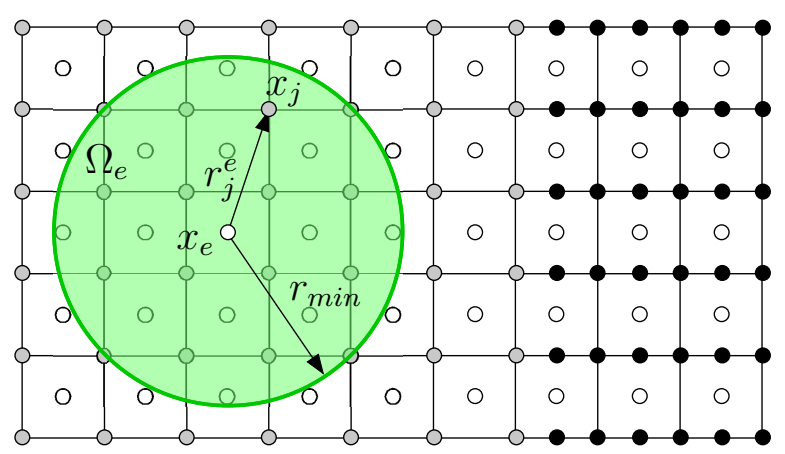

(a)

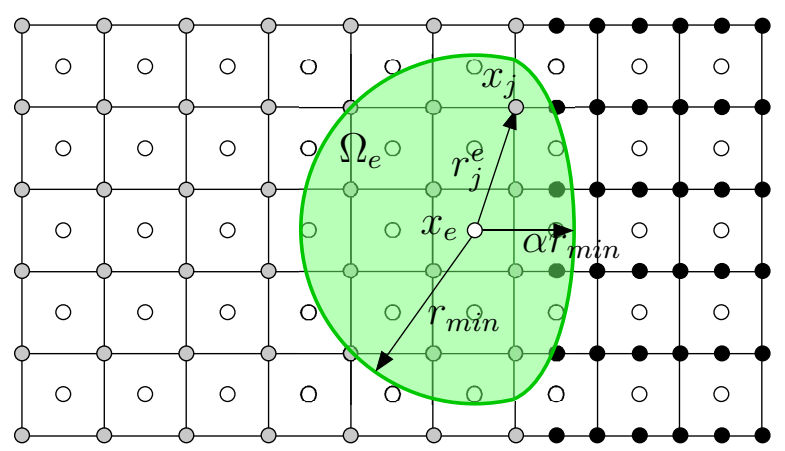

(b)

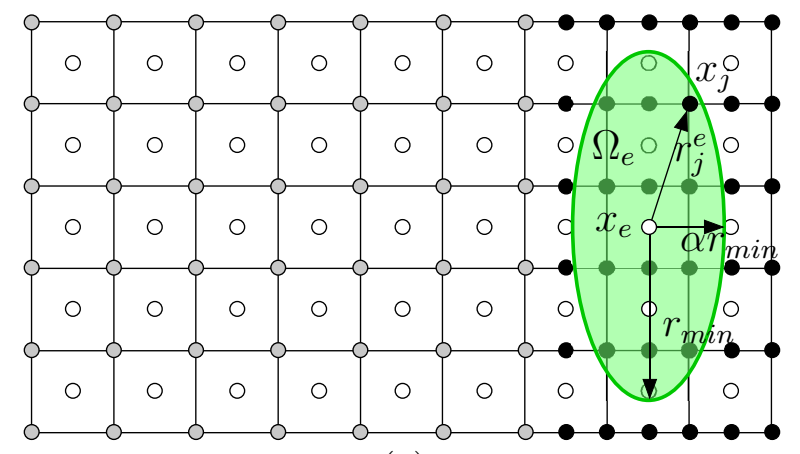

(c)

Figura 4.5: Exemplo do filtro de projeção com raio variável. 


$$
w_{j}^{e}=\left\{\begin{array}{cl}
\frac{r_{\min }-r_{j}^{e}}{r_{\min }} & \text { se } r \leq r_{\min } \\
0 & \text { se } r>r_{\min }
\end{array}\right.
$$

onde $w_{j}^{e}$ é o peso do nó $j$ do elemento $e, r_{j}^{e}$ define a distância entre o centróide do elemento $e$ até o nó pertencente à região de projeção $\Omega_{e}$. O cálculo de $r_{j}^{e}$ é definido por

$$
r_{j}^{e}= \begin{cases}x_{j}-x_{e} & x_{j} \in \Omega_{e}, x_{0} \leq x_{j} \leq x_{1}, x_{0} \leq x_{e} \leq x_{1} \\ \frac{\left(x_{j}-x_{1}\right)}{\alpha_{n}}+\left(x_{1}-x_{e}\right) & x_{j} \in \Omega_{e}, x_{1}<x_{j}, x_{0} \leq x_{e} \leq x_{1} \\ \frac{\left(x_{e}-x_{1}\right)}{\alpha_{n}}+\left(x_{1}-x_{j}\right) & x_{j} \in \Omega_{e}, x_{1}<x_{e}, x_{0} \leq x_{j} \leq x_{1} \\ \frac{\left(x_{j}-x_{e}\right)}{\alpha_{n}} & x_{j} \in \Omega_{e}, x_{1}<x_{j}, x_{1}<x_{e} \\ 0 & \text { caso contrário }\end{cases}
$$

Cada pseudo-densidade é calculada da seguinte forma:

$$
\rho_{e}=\frac{\sum_{j \in \Omega_{e}} w_{j}^{e} \rho_{j}^{e}}{\sum_{j \in \Omega_{e}} w_{j}^{e}}
$$

Como as pseudo-densidades de cada elemento são calculadas em função das variáveis de projeto nodais, o cálculo da sensibilidade deve ser modificado da seguinte forma:

$$
\frac{\partial(\cdot)}{\partial \rho_{j}^{e}}=\frac{\partial(\cdot)}{\partial \rho_{e}} \frac{\partial \rho_{e}}{\partial \rho_{j}}=\frac{\partial(\cdot)}{\partial \rho_{e}}\left(\frac{w_{j}^{e}}{\sum_{j \in \Omega_{e}} w_{j}^{e}}\right)
$$

\subsubsection{Cálculo das Sensibilidades}

Para utilizar o algoritmo MMA como otimizador, é necessário calcular as sensibilidades da função objetivo em função das variáveis de projeto. Nesta seção são apresentadas as expressões das sensibilidades em relação a uma variável de projeto genérica $\rho$ (a qual pode ser substituída pelas variáveis de projeto $d$ ). A potência elétrica apresentada na Eq. (4.5) pode ser escrita da seguinte forma:

$$
P=\frac{\left(\mathbf{L}_{\text {aux }}^{T} \boldsymbol{\Phi}\right)^{2}}{2 R}
$$

onde $\mathbf{L}_{a u x}$ é um vetor global auxiliar com um valor unitário positivo em um grau de liberdade de potencial elétrico qualquer em um dos eletrodos e um valor unitário negativo em um grau de liberdade de potencial elétrico qualquer no outro eletrodo. O valor negativo é devido a diferença de voltagem no cálculo da potência. Considerando que $\frac{\partial \mathbf{F}}{\partial \rho_{i}}=\mathbf{0}$ e $\mathbf{Q}=\mathbf{0}$, a derivada da função objetivo (Eq. (4.5)) é dada por (RUPP et al., 2009): 


$$
\begin{aligned}
\frac{\partial P}{\partial \rho_{i}} & =\left(\frac{\mathbf{L}_{a u x}^{T} \boldsymbol{\Phi}}{R}\right)\left\{\begin{array}{ll}
\mathbf{0} & \mathbf{L}_{\text {aux }}^{T}
\end{array}\right\}\left\{\begin{array}{c}
\frac{\partial \mathbf{U}}{\partial \rho_{i}} \\
\frac{\partial \boldsymbol{\Phi}}{\partial \rho_{i}}
\end{array}\right\}= \\
& =-\left(\frac{\mathbf{L}_{a u x}^{T} \boldsymbol{\Phi}}{R}\right)\left\{\begin{array}{ll}
\mathbf{0} & \mathbf{L}_{a u x}^{T}
\end{array}\right\} \mathbf{K}^{-1} \frac{\partial \mathbf{K}}{\partial \rho_{i}}\left\{\begin{array}{c}
\mathbf{U} \\
\boldsymbol{\Phi}
\end{array}\right\}= \\
& =-\left(\frac{\mathbf{L}_{a u x}^{T} \boldsymbol{\Phi}}{R}\right) \mathbf{L}_{a d j}^{T} \frac{\partial \mathbf{K}}{\partial \rho_{i}}\left\{\begin{array}{c}
\mathbf{U} \\
\boldsymbol{\Phi}
\end{array}\right\}
\end{aligned}
$$

onde

$$
\mathbf{K}=-\omega^{2}\left[\begin{array}{cc}
\mathbf{M} & \mathbf{0} \\
\mathbf{0} & \mathbf{0}
\end{array}\right]+i \omega\left(\left[\begin{array}{cc}
\mathbf{C} & \mathbf{0} \\
\mathbf{0} & \mathbf{0}
\end{array}\right]+\left[\begin{array}{cc}
\mathbf{0} & \mathbf{0} \\
\mathbf{0} & \mathbf{K}_{R}
\end{array}\right]\right)+\left[\begin{array}{ll}
\mathbf{K}_{\mathbf{u u}} & \mathbf{K}_{\mathbf{u} \phi} \\
\mathbf{K}_{\mathbf{u} \phi} & \mathbf{K}_{\phi \phi}
\end{array}\right]
$$

onde

$$
\frac{\partial \mathbf{K}}{\partial \rho_{i}}=\sum_{N}\left(-\omega^{2} \frac{\partial}{\partial \rho_{i}}\left[\begin{array}{rr}
\mathbf{M}^{e} & \mathbf{0} \\
\mathbf{0} & \mathbf{0}
\end{array}\right]+i \omega \frac{\partial}{\partial \rho_{i}}\left[\begin{array}{cc}
\mathbf{C}^{e} & \mathbf{0} \\
\mathbf{0} & \mathbf{0}
\end{array}\right]+\frac{\partial}{\partial \rho_{i}}\left[\begin{array}{ll}
\mathbf{K}_{\mathbf{u u}}^{e} & \mathbf{K}_{\mathbf{u} \phi}^{e} \\
\mathbf{K}_{\mathbf{u} \phi}^{e} & \mathbf{K}_{\phi \phi}^{e}
\end{array}\right]\right)
$$

O parâmetro $N$ é o número de elementos finitos. O vetor adjunto $\mathbf{L}_{a d j}$ é utilizado para evitar a inversão da matriz $\mathbf{K}$, a qual é calculada do seguinte sistema linear:

$$
\mathbf{K L}_{a d j}=\left\{\begin{array}{c}
\mathbf{0} \\
\mathbf{L}_{a u x}
\end{array}\right\}
$$

Este vetor adjunto é calculado apenas uma vez por iteração, reduzindo assim o custo computacional. Uma vez que as pseudo-densidades de cada elemento são calculadas a partir das variáveis de projeto, o cálculo das sensibilidades deve ser modificado da seguinte forma:

$$
\frac{\partial c}{\partial \rho_{j}^{e}}=\frac{\partial c}{\partial \rho_{e}} \frac{\partial \rho_{e}}{\partial \rho_{j}}=\frac{\partial c}{\partial \rho_{e}}\left(\frac{w_{j}^{e}}{\sum_{j \in \Omega_{e}} w_{j}^{e}}\right)
$$

O fluxograma do algoritmo de otimização é mostrado na Fig. 4.6. O programa é implementado no software MATLAB.

\subsection{Exemplo Numérico}

O domínio de projeto adotado é mostrado na Fig. 4.7, cujas dimensões são $100 \times$ $20 \mathrm{~mm}$. As camadas piezelétricas são conectadas em paralelo no circuito elétrico (ver Fig. 4.8) (ZHU; WORTHINGTON; NJUGUNA, 2009). O resistor acoplado aos eletrodos, que representa a carga a ser alimentada eletricamente, é de $1 \mathrm{k} \Omega$. Há uma força harmônica 


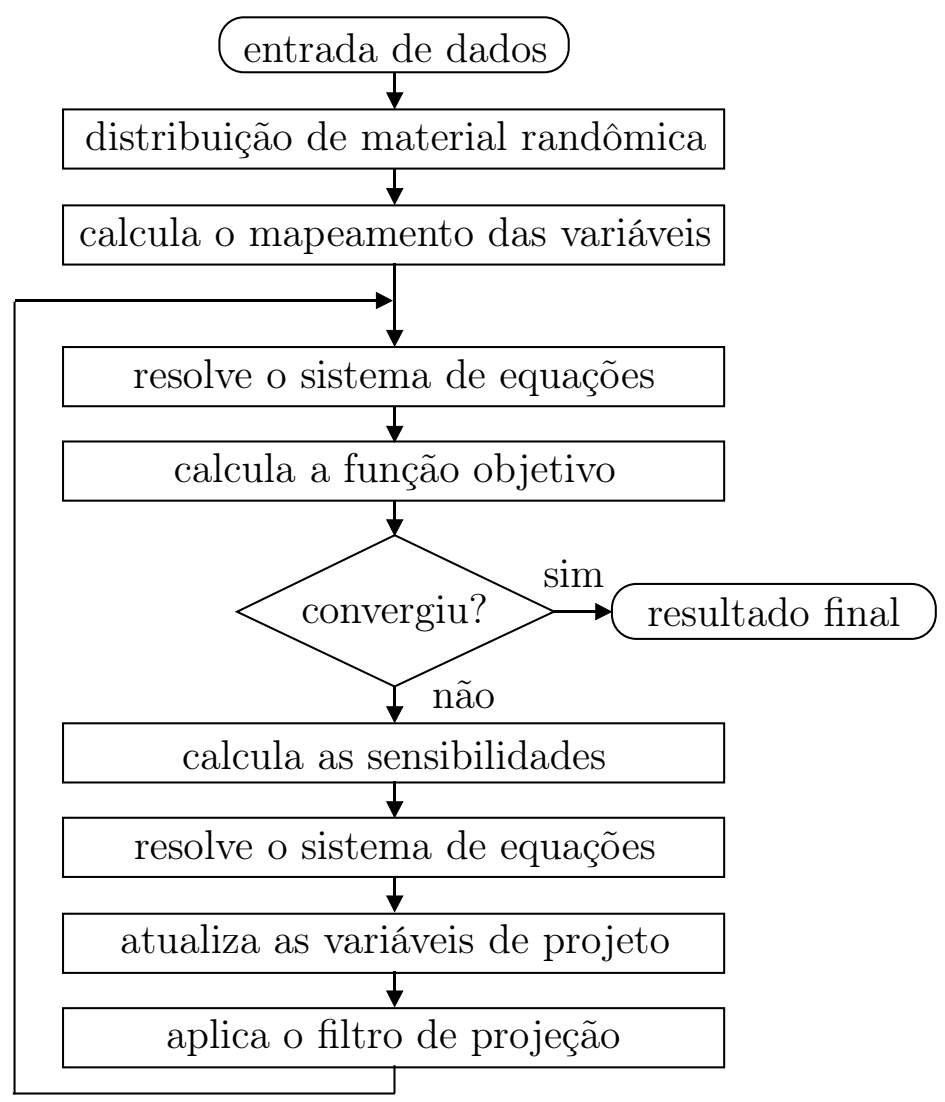

Figura 4.6: Fluxograma do algoritmo de otimização.

unitária aplicada na extremidade do domínio de projeto, com uma frequência de excitação de $1 \mathrm{kHz}$. Assume-se o estado plano de deformações e a simetria em relação ao eixo $x$. Os materiais adotados são PZT-5A e epóxi, cujas propriedades estão listadas na Tabela 2.4. O parâmetro de amortecimento $\beta$ tem o valor de $10^{-8}$ e a malha de elementos finitos é discretizada em $150 \times 30$ elementos quadriláteros bilineares de 4 nós. As sensibilidades são calculadas utilizando o método adjunto, conforme mostrado na seção anterior. Os coeficientes de penalização adotados tem valores iguais a $p_{c}=2, p_{e}=2$ e $p_{\varepsilon}=4$, de acordo com as duas condições para que haja uma convergência estável do problema de otimização (KIM et al., 2010).

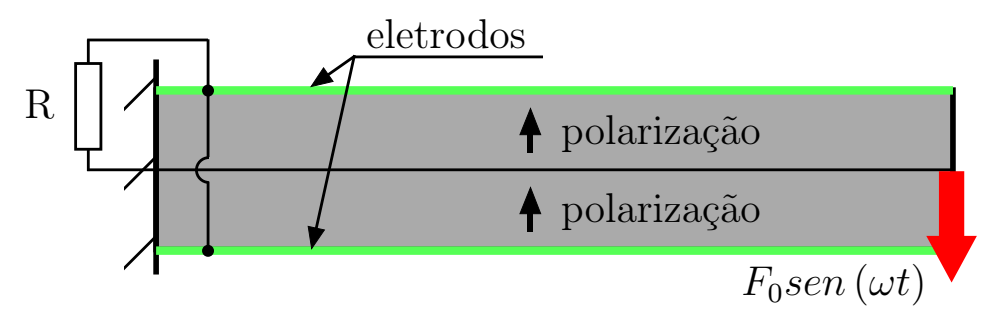

Figura 4.7: Domínio de projeto adotado.

O projeto de estruturas dinâmicas pode apresentar muitos mínimos locais, dificultando a convergência da solução. Portanto, a idéia principal deste estudo é avaliar a influência da gradação geométrica no projeto de coletores de energia. Para isso, o procedimento adotado consiste de três domínios de projeto distintos, conforme descrito a seguir. 


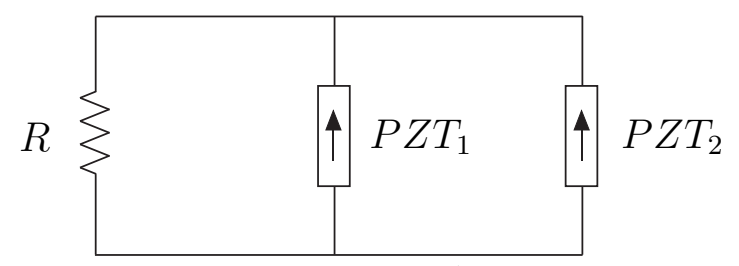

Figura 4.8: Circuito elétrico do modelo adotado (a seta indica o sentido da polarização piezelétrica).

\subsubsection{Caso 1: Sem repetição de padrões}

A Fig. 4.9 mostra o primeiro caso adotado, onde não é aplicada nenhuma repetição de padrões, e serve como referência para os outros dois casos. Este domínio tem o maior espaço de soluções, uma vez que os espaço de soluções dos outros casos está contido neste espaço.

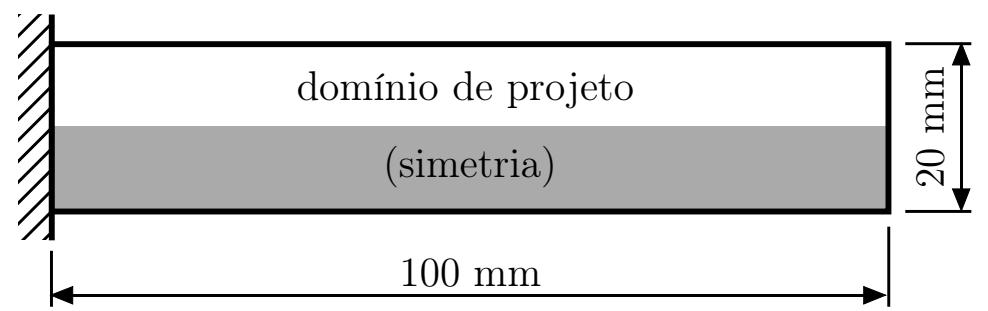

Figura 4.9: Caso 1: sem repetição de padrões.

\subsubsection{Caso 2: Com repetição de padrão}

A Fig. 4.10 mostra o segundo caso, onde é imposta a repetição de padrão ao longo do domínio, sem gradação entre as repetições, isto é, todas as repetições tem os mesmo tamanho. Este caso possui um espaço de soluções menor do que o caso anterior, pois todas as soluções disponíveis neste caso são limitadas àquelas que possuem repetições de padrão ao longo do domínio. Neste caso, as variáveis de projeto estão concentradas no domínio de projeto indicado na Fig. 4.10 e os valores das pseudo-densidades sob o domínio são simétricas em relação ao eixo $x$. As pseudo-densidades nas repetições são mapeadas em função do padrão.

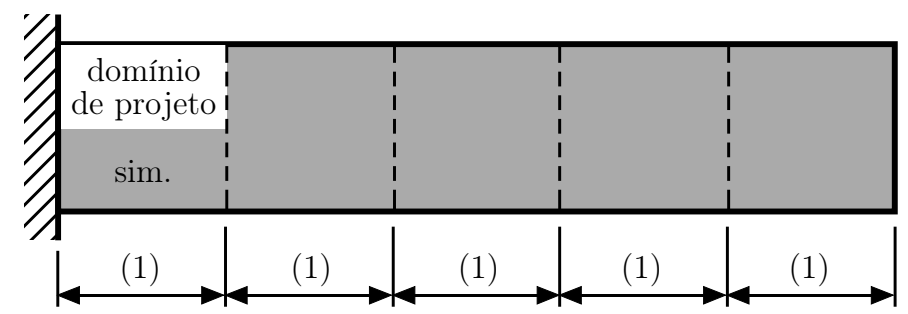

Figura 4.10: Caso 2: com repetição de padrão. 


\subsubsection{Caso 3: Com gradação geométrica}

A Fig. 4.11 mostra o terceiro caso adotado, onde é imposta gradação geométrica no domínio. A gradação do exemplo apresentado neste capítulo segue a proporção de 5, 4, 3,2 , e 1 , isto é, o primeiro padrão é 5 vezes maior do que o último, o segundo é 4 vezes maior e assim por diante. Diversas combinações de valores de gradação foram testadas à priori e essa combinação adotada é a que gerou um melhor desempenho.

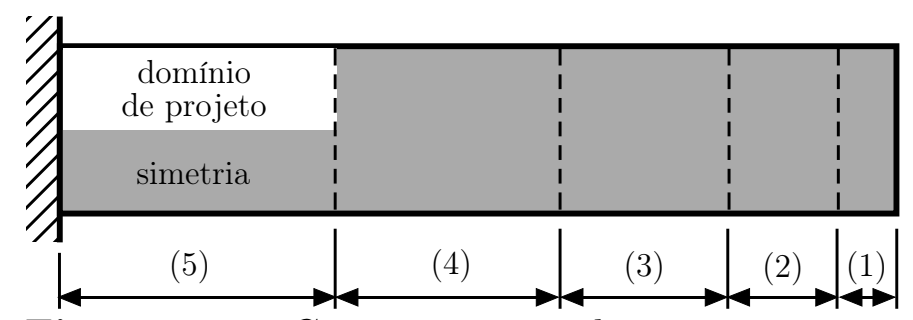

Figura 4.11: Caso 3: com gradação geométrica.

\subsubsection{Resultados e Discussões}

Conforme mostrado nos capítulos anteriores, materiais piezocompósitos podem ter melhores desempenhos do que materiais piezelétricos puros. Entretanto, para efeito de comparação, primeiramente é simulado um modelo formado inteiramente por PZT-5A, mostrado na Fig. 4.15a. A primeira frequência de ressonância e a potência elétrica gerada no resistor são $1020 \mathrm{~Hz}$ e $100 \mu W$, quando a estrutura é excitada a $1000 \mathrm{~Hz}$.

Primeiramente, foram investigadas diferentes distribuições iniciais de material, tais como distribuição uniforme de materiais, distribuições lineares ou gradações circulares ao longo do domínio de projeto, distribuições randômicas, entre outras. Após diversas tentativas, o procedimento adotado neste estudo consistiu em utilizar um conjunto de 20 distribuições iniciais randômicas de material. Destas 20 simulações, aproximadamente metade das simulações convergiram para um mesmo resultado, o qual gerou a maior potência elétrica para este domínio de projeto (sem repetição de padrões ou gradação geométrica). A outra metade das distribuições iniciais randômicas resultaram em diferentes topologias finais, gerando menos potência no resistor, isto é, convergiram a diferentes mínimos locais.

Para exemplificar a existência de múltiplos mínimos locais, a Fig. 4.12 mostra três exemplos de resultados obtidos a partir de diferentes distribuições iniciais de material. A partir dessa figura é possível ver que, embora as topologias obtidas diferem-se drasticamente, as curvas das potências elétricas geradas em função da frequência de excitação são semelhantes. A partir dessa figura também é possível ver a melhor solução obtida sem repetição de padrões (representada pela linha cheia), gerando $163 \mu W$ de 
potência e tendo a primeira frequência de ressonância em $1001 \mathrm{~Hz}$, e a iteração seguinte (representada pela linha tracejada), com pequenas variações nos valores das variáveis de projeto. Esse exemplo mostra que a otimização dinâmica é bastante sensível em relação às variáveis de projeto, tendo um impacto negativo na convergência da solução e conduzindo o problema a mínimos locais. Esse fenômeno é comum em métodos de otimização baseados em gradientes. No caso exemplificado, a potência elétrica cai de $163 \mu W$ a menos de 10 $\mu W$, quando o modelo é excitado a $1 \mathrm{kHz}$ (linha tracejada).

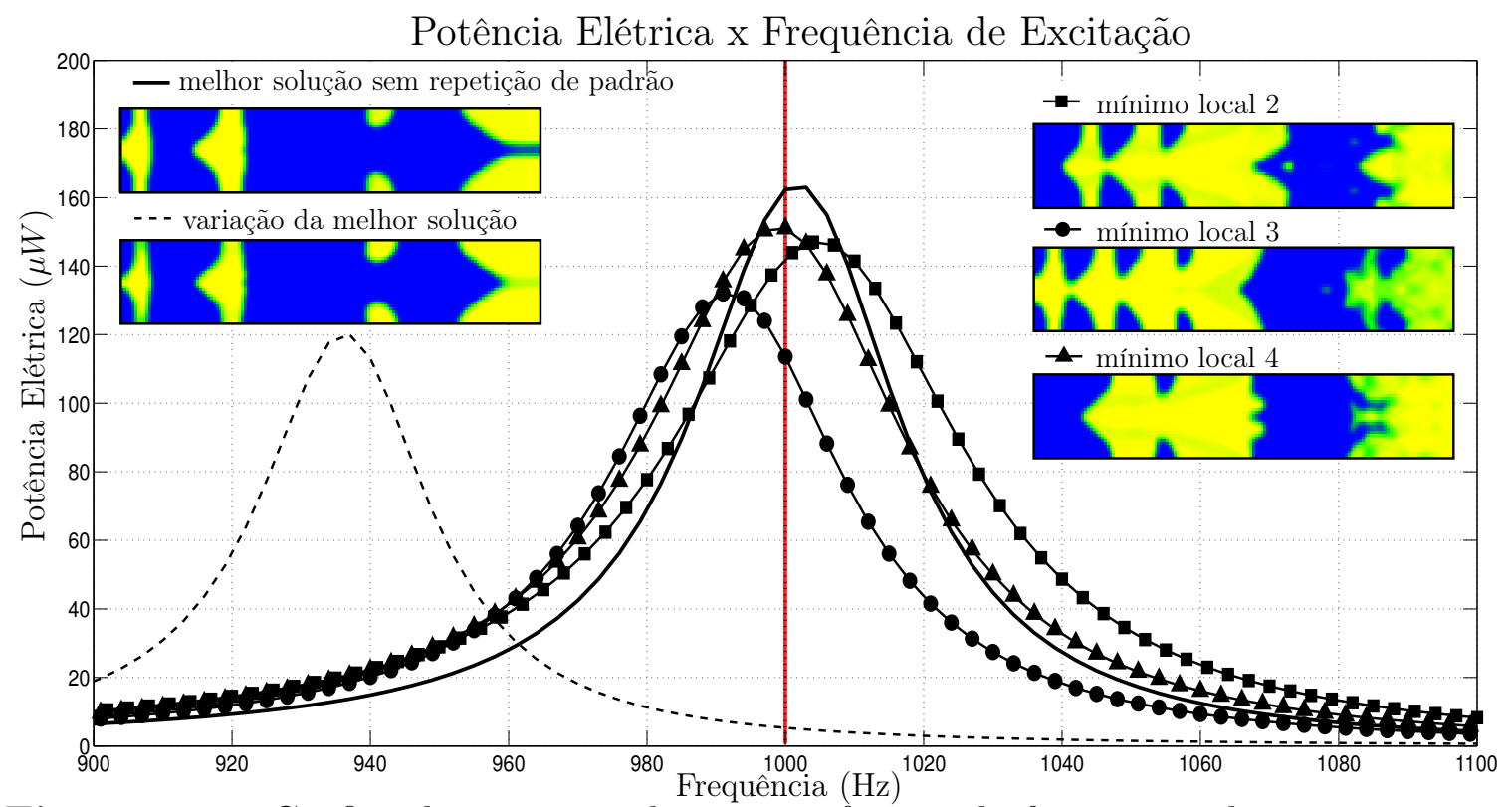

Figura 4.12: Gráfico da potência elétrica em função da frequência de excitação para diferentes mínimos locais, referente ao caso 1 (sem repetição de padrões).

Este mesmo estudo de sensibilidade em relação a pequenas variações nos valores das variáveis de projeto descrito anteriormente para o caso sem repetição de padrões é aplicado aos outros dois casos (com repetição de padrões e com gradação geométrica) - ver Fig. 4.13 e 4.14, respectivamente. Essas figuras mostram os gráficos das potências elétricas geradas em função da frequência de excitação para a solução otimizada de cada caso, juntamente com topologias levemente modificadas. A partir destas curvas é possível notar que as soluções apresentadas são menos sensíveis a pequenas modificações na topologia e, consequentemente, facilita a convergência do problema de otimização a um mínimo global.

O mesmo procedimento de utilizar um conjunto de 20 distribuições iniciais randômicas de material é aplicado aos outros dois casos e aproximadamente a mesma proporção (50\%) converge para as mesmas soluções em cada caso, as quais apresentam os melhores desempenhos para cada caso. As soluções otimizadas obtidas nos três casos são ilustradas na Fig. 4.15, juntamente com o modelo formado por PZT-5A puro, o qual serve como referência para os outros casos. Os valores da primeira frequência de ressonância, da potência elétrica gerada e do coeficiente de acoplamento eletromecânico estão listados ao 


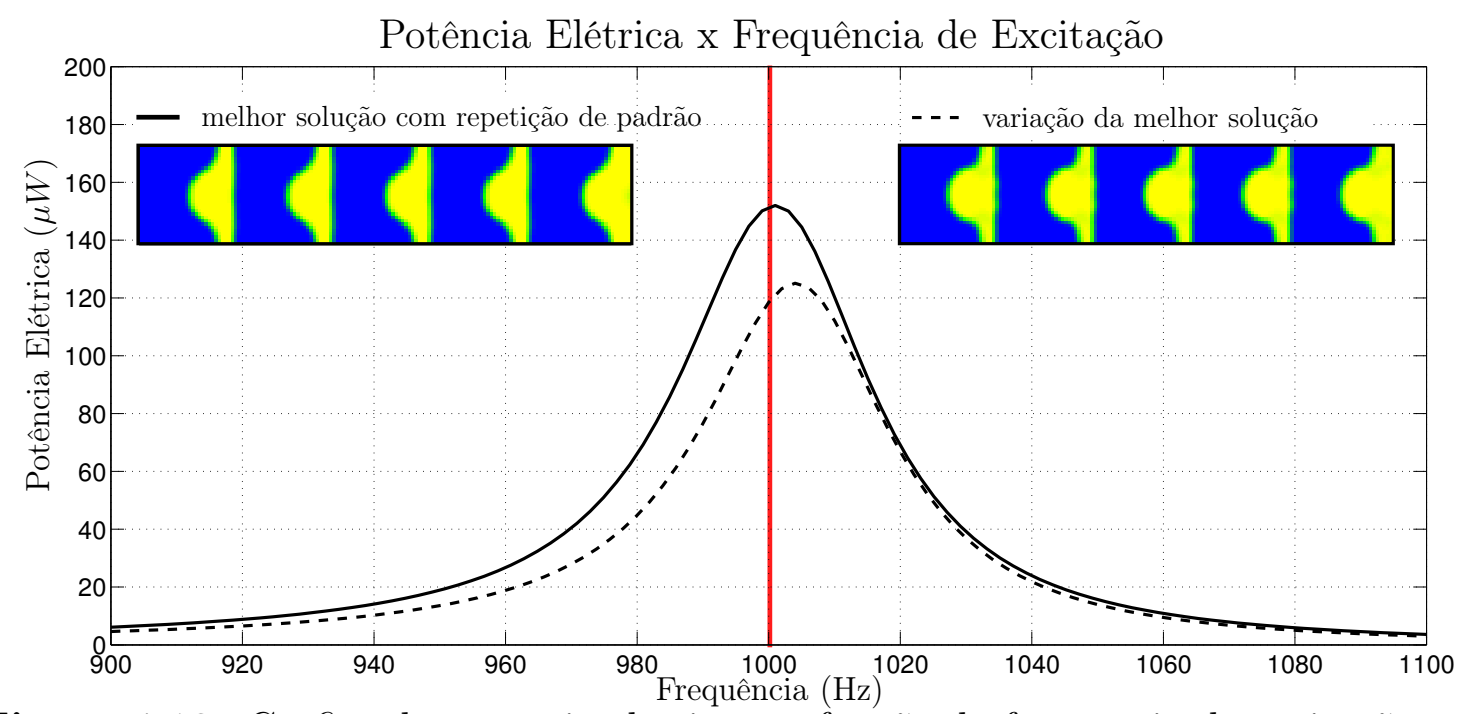

Figura 4.13: Gráfico da potência elétrica em função da frequência de excitação para diferentes mínimos locais, referente ao caso 1 (com repetição de padrão).

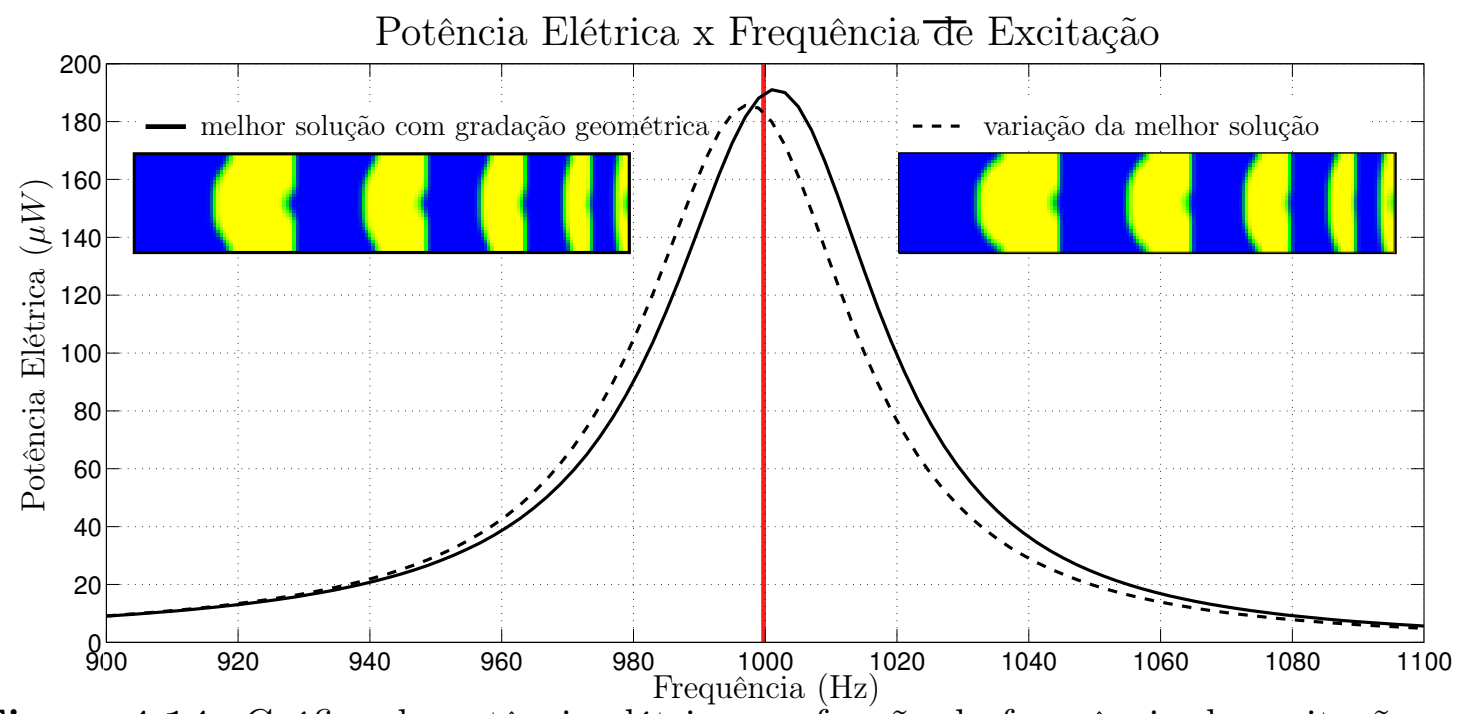

Figura 4.14: Gráfico da potência elétrica em função da frequência de excitação para diferentes mínimos locais, referente ao caso 1 (com gradação geométrica).

lado de cada caso.

Para o primeiro caso, onde não é considerada nenhuma restrição de padrões, a primeira frequência de ressonância é de $1001 \mathrm{~Hz}$, muito próxima da frequência de excitação, e a potência elétrica gerada é de $163 \mu W$, atingindo um ganho de $63 \%$ em relação ao PZT-5A puro. Para o segundo caso, onde é aplicada a repetição de padrões, a primeira frequência de ressonância é de $999 \mathrm{~Hz}$, a qual também é próxima a frequência de excitação, e a potência elétrica gerada é de $152 \mu \mathrm{W}$, atingindo um ganho de $52 \%$ em relação ao PZT-5A puro. Já o terceiro caso, onde é considerada a restrição de gradação geométrica, a primeira frequência de ressonância é de $995 \mathrm{~Hz}$, cujo valor é o mais distante da frequência de excitação dos três casos analisados, e a potência elétrica gerada é de $191 \mu W$, com um ganho de $91 \%$ em relação ao PZT-5A puro.

A partir desses resultados, é possível ver que o maior valor de potência gerada é obtida 
com o terceiro caso, onde é aplicada a gradação geométrica, mesmo que este caso tenha um espaço de soluções menor do que o primeiro caso. A principal conclusão é que, uma vez que o problema de otimização de estruturas dinâmicas tem muitos mínimos locais, a restrição de gradação geométrica modifica o espaço de soluções de forma a conduzir a solução a um melhor mínimo local.

A diferença entre as restrições de repetição de padrão e de gradação geométrica no resultado final pode ser observada através dos valores dos coeficientes de acoplamento eletromecânico $k$. Nos casos analisados, embora mais material piezelétrico seja necessário para converter energia, sua rigidez é maior do que do epóxi, diminuindo as deformações na estrutura e, consequentemente, menos energia é convertida. Já estruturas com menos material piezelétrico são mais flexíveis, porém convertem menos energia. Assim, há uma solução de compromisso entre a quantidade de material piezelétrico no domínio de projeto e a potência elétrica gerada. Comparando os valores dos coeficientes de acoplamento eletromecânico dos casos 2 e 3 (0,098 e 0,139, respectivamente), pode-se ver que a conversão de energia é mais eficiente para o terceiro caso, isto é, com restrição de gradação geométrica.

legenda: $\square$ PZT-5A $\square$ epóxi $\square$ eletrodos $\square$ terra

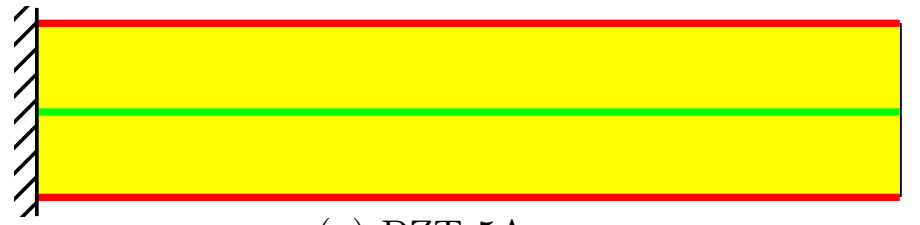

$\Rightarrow\left\{\begin{array}{l}1^{\circ} \text { modo }=1020 \mathrm{~Hz} \\ P=100 \mu W \\ k=0,329\end{array}\right.$

(a) PZT-5A puro

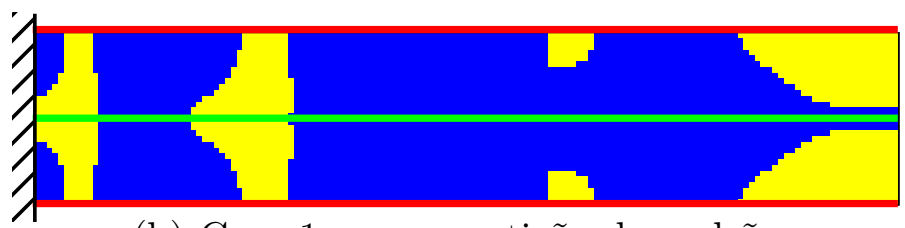

$\checkmark\left\{\begin{array}{l}1^{\circ} \text { modo }=1001 \mathrm{~Hz} \\ P=163 \mu W \\ k=0,102\end{array}\right.$

(b) Caso 1: sem repetição de padrões

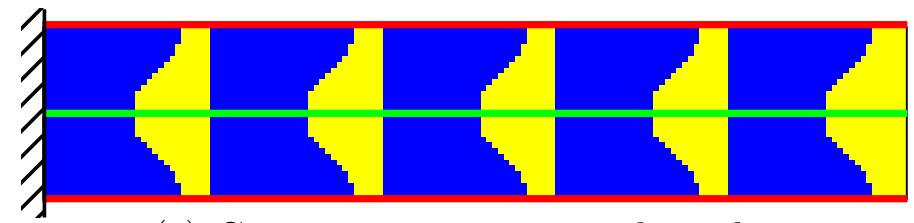

$\Rightarrow\left\{\begin{array}{l}1^{\circ} \text { modo }=999 \mathrm{~Hz} \\ P=152 \mu W \\ k=0,098\end{array}\right.$

(c) Caso 2: com repetição de padrão

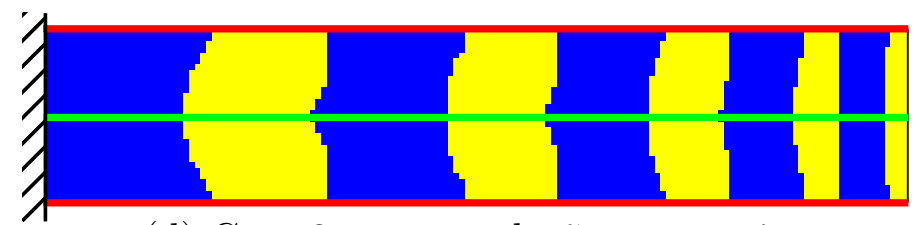

$\checkmark\left\{\begin{array}{l}1^{\circ} \operatorname{modo}=995 \mathrm{~Hz} \\ P=191 \mu W \\ k=0,139\end{array}\right.$

(d) Caso 3: com gradação geométrica

Figura 4.15: Melhor resultado para cada caso analisado. 
Outro importante aspecto a ser analisado é a resposta da potência elétrica gerada em função da frequência de ressonância, mostrada na Fig. 4.16. A partir deste gráfico nota-se claramente que, embora a primeira frequência de ressonância do resultado otimizado obtido com a restrição de gradação geométrica apresente uma diferença de $5 \mathrm{~Hz}$ em relação à frequência de excitação, este caso ainda gera a maior potência elétrica dos casos analisados.

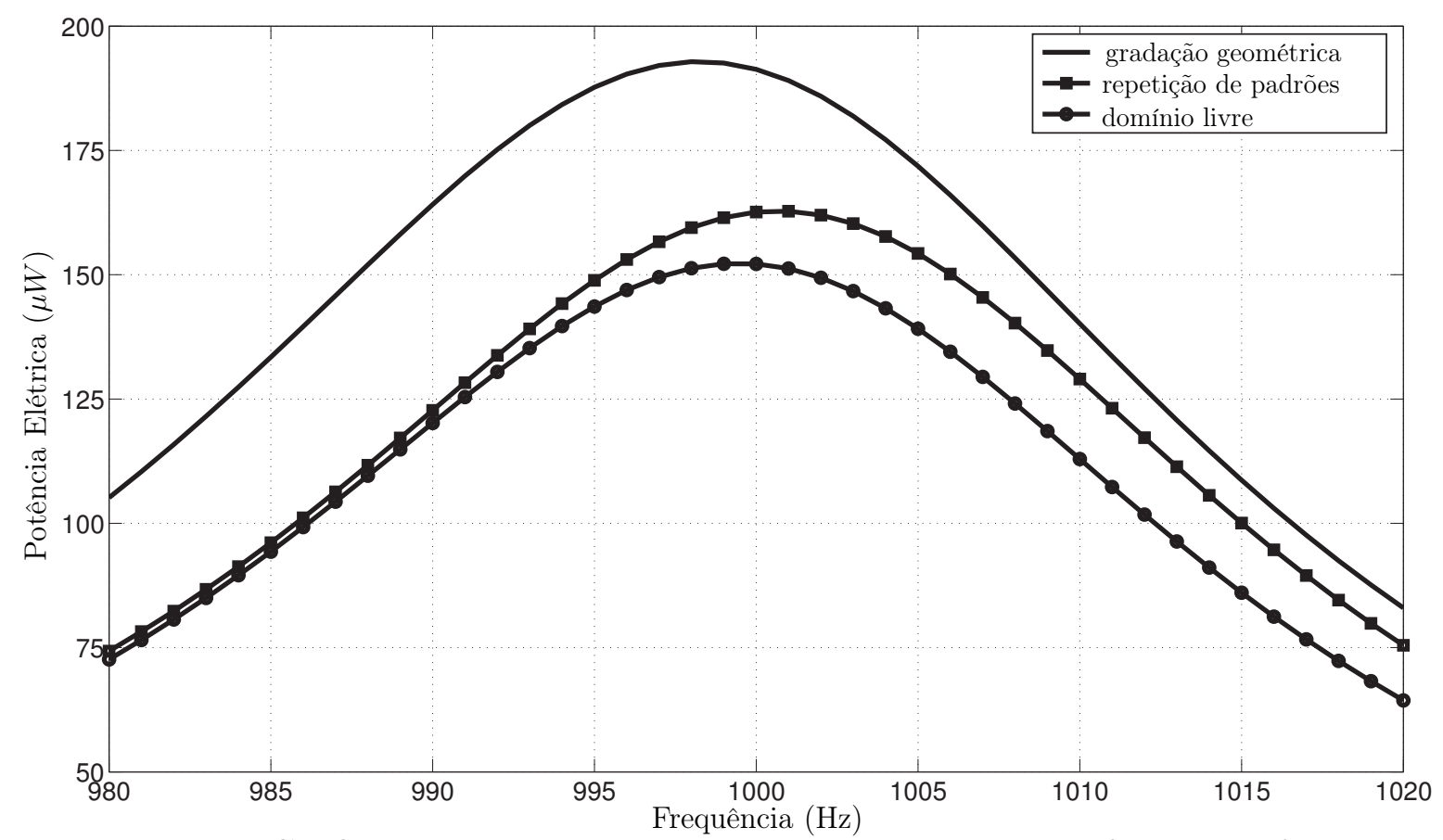

Figura 4.16: Gráfico da potência elétrica gerada no resistor em função da frequência de excitação.

\subsection{Considerações Finais}

Este capítulo apresentou a influência da gradação geométrica no projeto de dispositivos dinâmicos para coleta de energia baseados em estruturas de flexão, utilizando o MOT.

A maximização da potência elétrica gerada apresenta muitos mínimos locais, dificultando a obtenção do mínimo global. A partir dos resultados apresentados, conclui-se que a maior potência elétrica gerada é obtida com a restrição de gradação geométrica, mesmo que este caso apresente um espaço de soluções menor. Isto é possível pois, uma vez que a otimização de estruturas dinâmicas possuam muitos mínimos locais, a restrição de gradação geométrica modifica o espaço de soluções e torna a solução do problema mais estável, facilitando a obtenção de um melhor mínimo local.

A diferença da restrição de gradação geométrica em relação à repetição de padrões na maximização de potência elétrica gerada pode ser vista no coeficiente de acoplamento 
eletromecânico $k$. Comparando os valores de $k$ desses dois casos, nota-se que a conversão de energia é maior com a restrição de gradação geométrica. Entretanto, comparando-se os valores de $k$ dos resultados otimizados com o PZT-5A puro, nota-se que um maior valor de $k$ não implica necessariamente numa maior potência gerada.

A escolha do número de repetições e da gradação geométrica imposta ao longo do domínio de projeto foram baseadas em testes preliminares. Pode-se avaliar a influência das novas variáveis de projeto adicionadas ao projeto de estruturas dinâmicas piezelétricas, tais como o número de repetições de padrão e a gradação aplicada dessas repetições ao longo do domínio de projeto.

Neste estudo adotou-se um valor fixo do resistor acoplado aos eletrodos; entretanto, esse resistor também pode ser otimizado, isto é, pode-se encontrar o valor ótimo de $\mathrm{R}$ que gere a maior potência elétrica. 


\section{Conclusões Finais}

Este trabalho propõe metodologias sistemáticas para o projeto de materiais piezocompósitos com gradação funcional (MPGF) utilizando o Método de Otimização Topológica (MOT), tanto para aplicações quasi-estáticas, quanto para aplicações dinâmicas. Em aplicações quasi-estáticas de piezocompósitos assume-se que os comprimentos de onda de excitação são grandes o suficiente para que a estrutura detalhada da célula unitária seja desprezível e o material possa ser considerado como um novo meio homogêneo, com novas propriedades efetivas. Assim, uma dada excitação propaga-se através das pequenas variações do meio compósito, da mesma forma que se propaga na estrutura granular na escala micrométrica em uma cerâmica pura convencional. Entretanto, em aplicações dinâmicas, isto é, quando os comprimentos de onda não são grandes o suficiente em relação ao tamanho da célula unitária, o piezocompósito apresenta um comportamento dispersivo com reflexões ocorrendo dentro da célula unitária e, portanto, há a necessidade de se utilizar abordagens diferentes do caso homogêneo.

No estudo de projeto de MPGF empregados em aplicações quasi-estáticas, o método denominado por Otimização de Material Discreto (OMD) é utilizado para investigar a influência das direções de polarização piezelétrica. A partir dos exemplos de microestruturas obtidas nesse estudo, conclui-se que as direções de polarização variáveis na célula unitária são fatores importantes no projeto de MPGF e permitem obter microestruturas com melhores desempenhos. Foi também investigada a utilização de elementos poligonais e para os casos estudados conclui-se que, utilizando o mesmo número de elementos finitos, malhas poligonais não estruturadas conduzem o problema a melhores mínimos locais, quando comparadas a malhas quadriláteras estruturadas.

A capacidade do método de homogeneização de determinar microtensões mostra que, para piezocompósitos, valores de tensão efetivos calculados através de um modelo em escala macroscópica podem induzir a uma má seleção de materiais em projetos estruturais. Embora isso já seja conhecido na literatura, os resultados apresentados quantificam a diferença que pode ocorrer entre as tensões macro e microscópicas. Assim, os resultados mostram que as tensões microscópicas podem ser até 8 vezes maiores do que a tensão macroscópica, os quais tornam o material mais suscetível a microdefeitos, 
por exemplo. Outro aspecto importante é que concentrações de microtensões podem ser redistribuídas na célula unitária em função da gradação dos materiais. Portanto, a conclusão principal nesse tópico é que, embora as microestruturas discretas apresentadas apresentem valores maiores na função objetivo, estas também podem apresentar maiores valores de microtensões na célula unitária. Desse modo, para evitar falhas, estas estruturas suportarão menores carregamentos externos e, consequentemente, converterão menos energia mecânica em elétrica. Por outro lado, embora estruturas com gradação funcional apresentem valores menores da função objetivo, estas apresentam também menores valores de microtensões na célula unitária. Assim, podem suportar maiores carregamentos externos e, consequentemente, podem converter mais energia. Logo, há uma solução de compromisso entre a maximização do coeficiente de acoplamento eletromecânico e das concentrações de tensões microscópicas no interior da célula unitária.

No projeto de MPGF utilizados em aplicações fonônicas, os resultados obtidos a partir do modelo unidimensional mostram que o conceito de MGF pode ser aplicado para projetar materiais piezocompósitos fonônicos com prescrição de largura e posição de band gaps. A maximização de band gaps naturalmente gera topologias de material discretas, entretanto, o conceito de MGF pode ser utilizado para "sintonizar" os band gaps de acordo com os requisitos de cada aplicação.

Já utilizando o modelo bidimensional no projeto de MPGF fonônicos, são projetados diversos materiais piezocompósitos fonônicos visando a maximização de band gaps. Todas as topologias otimizadas obtidas pela metodologia de projeto proposta apresentam inclusões de PZT-5A em uma matriz de epóxi. Nem todos os intervalos entre as frequências de ressonância podem ser abertos, devido à simetria imposta no domínio de projeto. Alguns casos resultam em band gaps adicionais no intervalo de frequência analisado. Entretanto, estes band gaps adicionais são menores do que se esses mesmos band gaps fossem considerados como função objetivo. Dois exemplos diferentes de maximização de band gaps apresentaram células unitárias semelhantes, porém, com inclusões de PZT-5A em diferente escala, com diagramas de dispersão distintos. Isso mostra que o efeito de escala da topologia obtida tem grande influência no comportamento de materiais fonônicos. Além disso, analisando-se a influência de diferentes curvas de gradação de material entre as fases constituintes, conclui-se que as larguras dos band gaps são bastante sensíveis à curva de gradação de material. Nos resultados obtidos nesse estudo, quanto mais alto for o valor do coeficiente de penalização $q$ do filtro de projeção, maior é a largura do band gap. Esse efeito é devido à distribuição de material na célula unitária que se torna cada vez mais próxima de uma distribuição discreta para valores altos de $q$. É verdade que para valores pequenos de $q$ a distribuição de material tende a ser discreta também; entretanto, nos resultados obtidos nesse estudo esse efeito foi só observado para os valores 
maiores de $q$ adotados.

No projeto de dispositivos dinâmicos utilizando o MOT para coleta de energia baseados em estruturas de flexão, os resultados numéricos apresentados mostram que, para o domínio de projeto analisado, uma maior potência elétrica gerada pode ser obtida com a restrição de gradação geométrica, mesmo que este caso apresente um espaço de soluções menor. Isto é possível pois, uma vez que a otimização de estruturas dinâmicas possuam muitos mínimos locais, a restrição de gradação geométrica modifica o espaço de soluções e torna a solução do problema mais estável, facilitando a obtenção de um melhor mínimo local.

\subsection{Sugestões de Trabalhos Futuros}

Conforme mostrado no Capítulo 3, para se construir o diagrama de dispersão, deve-se variar a direção e comprimento do vetor de onda $\mathbf{k}$ e calcular as frequências de ressonância do modelo. À princípio, deveria-se calcular o diagrama de dispersão variando-se $\mathbf{k}$ em todas as direções da célula unitária mas, embora não tenha sido provado, a maioria dos pesquisadores alega que é possível caracterizar uma célula unitária completamente analisando apenas a primeira zona irredutível de Brillouin (SIGMUND; JENSEN, 2003), sem perda de informações. Assim, uma sugestão de trabalho futuro é investigar se a análise apenas da primeira zona de Brillouin é suficiente para a completa descrição do comportamento fonônico do material. Como a demonstração matemática desta condição pode ser extremamente complexa ou até impossível, uma alternativa é encontrar uma estrutura fonônica, possivelmente utilizando o MOT, cujo diagrama de dispersão analisado na primeira zona de Brillouin seja invalidado. Isso pode ser realizado da seguinte forma: dada uma estrutura de célula unitária qualquer, obter superfícies formadas pelos autovalores da Eq. (3.6) para diversos vetores de onda k, que cubram tanto a fronteira quanto o próprio interior da primeira zona de Brillouin. Então, formula-se uma função objetivo cujo foco seja obter um band gap menor para algum vetor de onda que reside no interior da primeira zona de Brillouin. Caso seja encontrada tal estrutura, a análise do diagrama de dispersão usualmente apresentado na literatura tornaria-se inválida, pois a verificação apenas de vetores de onda posicionados nas fronteiras da primeira zona de Brillouin seria insuficiente para descrever o comportamento dispersivo do material.

Em relação ao conceito de gradação geométrica aplicado ao MOT, pode ser avaliada a influência das novas variáveis de projeto adicionadas ao projeto de estruturas dinâmicas piezelétricas, tais como o número de repetições de padrão e a gradação aplicada a essas repetições ao longo do domínio de projeto. 
Um efeito interessante observado durante o projeto de materiais fonônicos é que nas frequências limitantes dos band gaps existem ondas estacionárias. Nestas regiões, o epóxi atua como um absorvedor de vibração e o PZT-5A atua como um corpo rígido. Este fenômeno ocorre devido à diferença de rigidez entre os materiais. Dada esta observação, novas combinações de materiais piezocompósitos podem ser investigadas, de forma que o PZT-5A tenha propriedades elásticas menos rígidas do que o outro material constituinte. Isso pode ser obtido unindo o PZT-5A com um material mais rígido, como por exemplo, o níquel, ou adicionando vazios na região do PZT-5A (ver Fig. 5.1). Assim, acredita-se que o PZT-5A atue como um absorvedor de vibrações, sofrendo grandes deformações e, consequentemente, convertendo mais energia mecânica em elétrica.

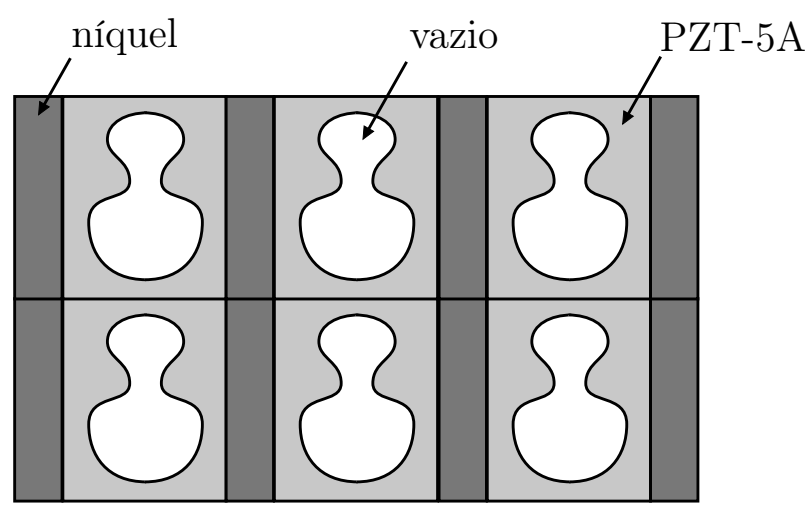

Figura 5.1: Exemplo de material onde a região piezelétrica é a menos rígida do piezocompósito.

O projeto de materiais fonônicos apresentado anteriormente motiva o estudo de projeto de estruturas de invisibilidade acústica utilizando o conceito de guias de ondas acústicas. As ondas acústicas, enquanto tentam se propagar em um meio de estruturas periódicas, podem sofrer diversas interferências destrutivas em certas faixas de frequência (band gaps), de forma que sejam completamente atenuadas (KUSHWAHA et al., 1993, 1994). Entretanto, adotando-se devidamente uma sequência de "defeitos" na estrutura periódica, é possível restringir a onda em determinados canais, ou dutos, obrigando-a a seguir um determinado caminho (SIGALAS, 1998). Este efeito é conhecido como guia de onda. Desse modo, em uma determinada frequência a propagação da onda ocorre apenas na direção desejada, seguindo as regiões de "defeito" da estrutura, uma vez que a propagação em outras direções é impedida devido às interferências destrutivas geradas pela periodicidade da estrutura.

Acredita-se que esse conceito de guias de onda pode ser utilizado no projeto de estruturas para invisibilidade acústica. A invisibilidade acústica é um novo campo de pesquisa, onde o objetivo principal é "blindar" um determinado objeto ou região, do ponto de vista acústico (CHEN; CHAN, 2007; CUMMER; SCHURIG, 2007; TORRENT; SANCHEZ-DEHESA, 2008). Em outras palavras, são estruturas capazes de minimizar 
possíveis interferências (por exemplo: reflexão, difração, dispersão) de ondas acústicas que seriam ocasionadas por um dado objeto qualquer, localizado em um meio propagante, de modo que a propagação da onda seja da forma mais semelhante possível comparado ao caso sem a presença desse objeto. 


\section{Referências}

ABDALLA, M.; FRECKER, M.; GURDAL, Z.; JOHNSON, T.; LINDNER, D. K. Design of a piezoelectric actuator and compliant mechanism combination for maximum energy efficiency. Smart Materials and Structures, v. 14, n. 6, p. 1421-1430, dez. 2005.

ABOUDI, J.; PINDERA, M. J.; ARNOLD, S. M. Higher-order theory for functionally graded materials. Composites Part B-Engineering, v. 30, n. 8, p. 777-832, 1999.

ADAMOWSKI, J. C.; MATUDA, M. Y.; BUIOCHI, F.; FURUKAWA, C. M.; SIGELMANN, R. A. Combination of analytical and finite element methods to obtain acoustic fields in complex structures driven by piezoelectric multi-layered transducers. 1997 IEEE Ultrasonics Symposium Proceedings, Vols 1 and 2, p. 955-958, 1997.

AGBOSSOU, A.; VIET, H. N.; PASTOR, J. Homogenization techniques and application to piezoelectric composite materials. International Journal of Applied Electromagnetics and Mechanics, v. 10, n. 5, p. 391-403, out. 1999.

AKDOGAN, E. K.; ALLAHVERDI, M.; SAFARI, A. Piezoelectric composites for sensor and actuator applications. IEEE Transactions on Ultrasonics, Ferroelectrics and Frequency Control, v. 52, n. 5, p. 746-775, maio 2005.

ALEXANDER, P. W.; BREI, D.; HALLORAN, J. W. The fabrication and material characterization of PZT based functionally graded piezoceramics. Smart Structures and Materials 2005: Smart Structures and Integrated Systems, Spie-int Soc Optical Engineering, v. 5764, p. 57-70, 2005.

ALLAIRE, G. Shape optimization by the homogenization method. New York: Applied mathematical sciences, 2002.

ALLAIRE, G.; JOUVE, F.; TOADER, A. M. Structural optimization using sensitivity analysis and a level-set method. Journal of Computational Physics, v. 194, n. 1, p. 363-393, fev. 2004.

ALLIK, H.; HUGHES, T. J. R. Finite element method for piezoelectric vibration. International Journal of Numerical Methods in Engineering, v. 2, p. 151-157, 1970.

ALMAJID, A.; TAYA, M.; HUDNUT, S. Analysis of out-of-plane displacement and stress field in a piezocomposite plate with functionally graded microstructure. International Journal of Solids and Structures, v. 38, n. 19, p. 3377-3391, 2001.

ALMAJID, A. A.; TAYA, M. 2D-Elasticity analysis of FGM piezo-laminates under cylindrical bending. Journal of Intelligent Material Systems and Structures, Sage Publications Ltd, v. 12, n. 5, p. 341-351, maio 2001.

ANDRADE, M. A. B.; ALVAREZ, N. P.; BUIOCHI, F.; NEGREIRA, C.; ADAMOWSKI, J. C. Analysis of 1-3 piezocomposite and homogeneous piezoelectric rings for power ultrasonic transducers. Journal of the Brazilian Society of Mechanical Sciences and Engineering, v. 31, n. 4, p. 312-318, out. 2009. 
ANSI/IEEE. An American national standard - IEEE standard on piezoelectricity. IEEE Transactions on Sonics and Ultrasonics, v. 31, n. 2, p. 1-55, 1984.

ANTON, S. R.; SODANO, H. A. A review of power harvesting using piezoelectric materials (2003-2006). Smart Materials and Structures, v. 16, n. 3, p. R1-R21, 2007.

AULD, B. A. Acoustic Fields and Waves in Solids. 2 ed.. ed. [S.l.]: Krieger Pub Co, 1990.

AVELLANEDA, M.; SWART, P. J. The role of matrix porosity and poisson's ratio in the design of high-sensitivity piezocomposite transducers. Adaptive Structures and Composite Materials: Analysis and Application - ASME Aerospace Division AD, v. 45, p. 59-66, 1994.

BATHE, K. J. Finite Element Procedures. [S.l.]: Englewood Cliffs, N.J., 1996.

BEEBY, S. P.; TUDOR, M. J.; WHITE, N. M. Energy harvesting vibration sources for microsystems applications. Measurement Science and Technology, v. 17, n. 12, p. R175-R195, 2006.

BENDSØE, M. P.; KIKUCHI, N. Generating optimal topologies in structural design using a homogenization method. Computer Methods in Applied Mechanics and Engineering, Elsevier Science Sa Lausanne, v. 71, n. 2, p. 197-224, nov. 1988.

BENDSØE, M. P.; SIGMUND, O. Material interpolation schemes in topology optimization. Archive of Applied Mechanics, v. 69, n. 9-10, p. 635-654, nov. 1999.

BENDSØE, M. P.; SIGMUND, O. Topology Optimization - Theory, Methods and Applications. New York, U.S.A.: Springer, 2003.

BOLANDER, J. E.; SAITO, S. Fracture analyses using spring networks with random geometry. Engineering Fracture Mechanics, v. 61, n. 5-6, p. 569-591, 1998.

BOURDIN, B. Filters in topology optimization. International Journal for Numerical Methods in Engineering, John Wiley and Sons Ltd, v. 50, n. 9, p. 2143-2158, mar. 2001.

BRUNS, T. E.; TORTORELLI, D. A. Topology optimization of non-linear elastic structures and compliant mechanisms. Computer Methods In Applied Mechanics and Engineering, Elsevier Science Sa, v. 190, n. 26-27, p. 3443-3459, 2001.

BUEHLER, M. J.; BETTIG, B.; PARKER, G. G. Topology optimization of smart structures using a homogenization approach. Journal of Intelligent Material Systems and Structures, v. 15, n. 8, p. 655-667, ago. 2004.

CARBOnARI, R. C.; SILVA, E. C. N.; NISHIWAKI, S. Design of piezoelectric multi-actuated microtools using topology optimization. Smart Materials and Structures, v. 14, n. 6, p. 1431-1447, 2005.

CARBONARI, R. C.; SILVA, E. C. N.; NISHIWAKI, S. Optimum placement of piezoelectric material in piezoactuator design. Smart Materials and Structures, v. 16, n. 1, p. 207-220, 2007.

CARBOnARI, R. C.; SILVA, E. C. N.; PAUlinO, G. H. Topology optimization design of functionally graded bimorph-type piezoelectric actuators. Smart Materials and Structures, v. 16, p. 2607-2620, 2007. 
CARBONARI, R. C.; SILVA, E. C. N.; PAUlinO, G. H. Multi-actuated functionally graded piezoelectric micro-tools design: A multiphysics topology optimization approach. International Journal for Numerical Methods in Engineering, v. 77, n. 3, p. 301-336, jan. 2009 .

CARDOSO, E.; FONSECA, J. S. O. Complexity control in the topology optimization of continuum structures. Journal of the Brazilian Society of Mechanical Sciences and Engineering, v. 25, n. 3, p. 293-301, 2003.

CHALlAGUlLA, K. S.; VENKATESH, T. A. Electromechanical response of 2-2 layered piezoelectric composites: A micromechanical model based on the asymptotic homogenization method. Philosophical Magazine, v. 89, n. 14, p. PII 911166965, 2009.

CHALLIS, V. J. A discrete level-set topology optimization code written in matlab. Structural and Multidisciplinary Optimization, v. 41, n. 3, p. 453-464, abr. 2010.

CHEN, H.; CHAN, C. T. Acoustic cloaking in three dimensions using acoustic metamaterials. Applied Physics Letters, v. 91, n. 18, p. 183518, 2007.

CHEN, S. K.; GONELlA, S.; CHEN, W.; LIU, W. K. A level set approach for optimal design of smart energy harvesters. Computer Methods in Applied Mechanics and Engineering, v. 199, n. 37-40, p. 2532-2543, 2010.

CHERKAEV, A. Variational methods for structural optimization. New York: Springer, 2000 .

CHU, D. N.; XIE, Y. M.; HIRA, A.; STEVEN, G. P. Evolutionary structural optimization for problems with stiffness constraints. Finite Elements In Analysis and Design, Elsevier Science Bv, v. 21, n. 4, p. 239-251, abr. 1996.

COOK-CHENnAUlt, K. A.; THAMBI, N.; SASTRY, A. M. Powering MEMS portable devices - A review of non-regenerative and regenerative power supply systems with special emphasis on piezoelectric energy harvesting systems. Smart Materials and Structures, v. 17, n. 4, p. 043001, 2008.

COX, S. J.; DOBSON, D. C. Maximizing band gaps in two-dimensional photonic crystals. SIAM Journal on Applied Mathematics, v. 59, n. 6, p. 2108-2120, 1999.

CRUMM, A. T.; HALLORAN, J. W. Fabrication of microconfigured multicomponent ceramics. Journal of the American Ceramic Society, v. 81, n. 4, p. 1053-1057, 1998.

CRUMM, A. T.; HAllorAn, J. W.; SIlVA, E. C. N.; ESPINOSA, F. M. de. Microconfigured piezoelectric artificial materials for hydrophones. Journal of Materials Science, v. 42, n. 11, p. 3944-3950, 2007.

CUMMER, S. A.; SCHURIG, D. One path to acoustic cloaking. New Journal of Physics, v. 9, p. $45,2007$.

DAHL, J.; JENSEN, J. S.; SIGMUND, O. Topology optimization for transient wave propagation problems in one dimension design of filters and pulse modulators. Structural and Multidisciplinary Optimization, v. 36, n. 6, p. 585-595, 2008.

DIAZ, A.; SIGMUND, O. Checkerboard patterns in layout optimization. Structural Optimization, Springer Verlag, v. 10, n. 1, p. 40-45, ago. 1995. 
DIJK, N. P. van; LANGELAAR, M.; KEULEN, F. van. A discrete formulation of a discrete level-set method treating multiple constraints. In: Proceedings of $8^{\text {th }}$ World Congress on Structural and Multidisciplinary Optimization. [S.l.: s.n.], 2009.

DONOSO, A.; BELLIDO, J. C. Systematic design of distributed piezoelectric modal sensors/actuators for rectangular plates by optimizing the polarization profile. Structural and Multidisciplinary Optimization, v. 38, n. 4, p. 347-356, maio 2009.

DONOSO, A.; SIGMUND, O. Optimization of piezoelectric bimorph actuators with active damping for static and dynamic loads. Structural and Multidisciplinary Optimization, v. 38, n. 2, p. 171-183, abr. 2009.

DU, Q.; EMELIANENKO, M.; JU, L. L. Convergence of the lloyd algorithm for computing centroidal voronoi tessellations. Siam Journal on Numerical Analysis, v. 44, n. 1, p. 102-119, 2006.

DU, Q.; FABER, V.; GUNZBURGER, M. Centroidal voronoi tessellations: Applications and algorithms. Siam Review, v. 41, n. 4, p. 637-676, dez. 1999.

EL-SABBAGH, A.; BAZ, A. Maximization of the harvested power from piezoelectric bimorphs with multiple electrodes under dynamic excitation. Finite Elements in Analysis and Design, v. 47, n. 11, p. 1232-1241, nov. 2011.

ELKA, A.; BUCHER, I. Optimal electrode shaping for precise modal electromechanical filtering. Structural and Multidisciplinary Optimization, v. 38, n. 6, p. 627-641, 2009.

ERTURK, A.; RENNO, J. M.; INMAN, D. J. Modeling of piezoelectric energy harvesting from an l-shaped beam-mass structure with an application to uavs. Journal of Intelligent Material Systems and Structures, v. 20, n. 5, p. 529-544, mar. 2009.

ESCHENAUER, H. A.; KOBELEV, V. V.; SCHUMACHER, A. Bubble method for topology and shape optimization of structures. Structural Optimization, Springer Verlag, v. 8 , n. 1 , p. 42-51, ago. 1994.

ESQUIVELSIRVENT, R.; COCOLETZI, G. H. Band-structure for the propagation of elastic-waves in superlattices. Journal of the Acoustical Society of America, v. 95, n. 1, p. 86-90, jan. 1994.

GALKA, A.; TELEGA, J. J.; WOJNAR, R. Homogenization and thermopiezoelectricity. Mechanics Research Communications, v. 19, n. 4, p. 315-324, 1992.

GIBIANSKY, L. V.; SIGMUND, O. Multiphase composites with extremal bulk modulus. Journal of the Mechanics and Physics of Solids, v. 48, n. 3, p. 461-498, 2000.

GIBIANSKY, L. V.; TORQUATO, S. Rigorous link between the conductivity and elastic-moduli of fiber-reinforced composite-materials. Philosophical Transactions of the Royal Society of London Series A-Mathematical Physical and Engineering Sciences, v. 353, n. 1702 , p. 243-278, 1995.

GIBIANSKY, L. V.; TORQUATO, S. On the use of homogenization theory to design optimal piezocomposites for hydrophone applications. Journal of the Mechanics and Physics of Solids, v. 45, n. 5, p. 689-708, 1997.

GIBIANSKY, L. V.; TORQUATO, S. Optimal design of 1-3 composite piezoelectrics. Structural Optimization, v. 13, n. 1, p. 23-38, 1997. 
GIBIANSKY, L. V.; TORQUATO, S. Matrix laminate composites: Realizable approximations for the effective moduli of piezoelectric dispersions. Journal of Materials Research, v. 14, n. 1, p. 49-63, 1999.

GOFFAUX, C.; VIGNERON, J. P. Theoretical study of a tunable phononic band gap system. Physical Review B, v. 64, n. 7, p. 075118, ago. 2001.

GONELLA, S.; TO, A. C.; LIU, W. K. Interplay between phononic bandgaps and piezoelectric microstructures for energy harvesting. Journal of the Mechanics and Physics of Solids, v. 57, n. 3, p. 621-633, 2009.

GUEDES, J. M.; KIKUCHI, N. Preprocessing and postprocessing for materials based on the homogenization method with adaptive finite-element methods. Computer Methods in Applied Mechanics and Engineering, v. 83, n. 2, p. 143-198, out. 1990.

HA, Y.; CHO, S. Design sensitivity analysis and topology optimization of eigenvalue problems for piezoelectric resonators. Smart Materials and Structures, v. 15, n. 6, p. 1513-1524, dez. 2006.

HABER, R. B.; JOG, C. S.; BENDSØE, M. P. A new approach to variable-topology shape design using a constraint on perimeter. Structural and Multidisciplinary Optimization, v. 11, p. 1-12, 1996.

HAFTKA, R. T.; GüRDAL, Z. Elements of Structural Optimization - Solid Mechanics and its Applications. Dordrecht, Netherlands: Kluwer Academic Publishers, 1993.

HALKJAER, S.; SIGMUND, O.; JENSEN, J. S. Inverse design of phononic crystals by topology optimization. Zeitschrift Fur Kristallographie, v. 220, n. 9-10, p. 895-905, 2005.

HALKJAER, S.; SIGMUND, O.; JENSEN, J. S. Maximizing band gaps in plate structures. Structural and Multidisciplinary Optimization, v. 32, p. 263-275, 2006.

HASHIN, Z.; SHTRIKMAN, S. A variational approach to the theory of the elastic behaviour of multiphase materials. Journal of the Mechanics and Physics of Solids, v. 11, n. 2, p. 127-140, 1963.

HASSANI, B.; HINTON, E. A review of homogenization and topology optimization i - homogenization theory for media with periodic structure. Computers and Structures, v. 69, n. 6 , p. $707-717,1998$.

HASSANI, B.; HINTON, E. A review of homogenization and topology optimization ii - analytical and numerical solution of homogenization equations. Computers and Structures, v. 69, n. 6, p. 719-738, 1998.

HASSANI, B.; HINTON, E. A review of homogenization and topology optimization iii topology optimization using optimality criteria. Computers and Structures, v. 69, n. 6, p. $739-756,1998$.

HAUKE, T.; KOUVATOV, A.; STEINHAUSEN, R.; SEIFERT, W.; BEIGE, H.; LANGHAMMER, H. T.; ABICHT, H. P. Bending behavior of functionally gradient materials. Ferroelectrics, v. 238, n. 1-4, p. 759-766, 2000.

HLADKYHENNION, A. C.; DECARPIGNY, J. N. Analysis of the scattering of a plane acoustic-wave by a doubly periodic structure using the finite-element method application to alberich anechoic coatings. Journal of the Acoustical Society of America, v. 90, n. 6, p. 3356-3367, 1991. 
HOU, Z. L.; FU, X. J.; LIU, Y. Y. Acoustic wave in a two-dimensional composite medium with anisotropic inclusions. Physics Letters A, v. 317, n. 1-2, p. 127-134, out. 2003.

HOU, Z. L.; WU, F. G.; LIU, Y. Phononic crystals containing piezoelectric material. Solid State Communications, v. 130, n. 11, p. 745-749, 2004.

HOY, C. V.; BARDA, A.; GRIFFITH, M.; HALLORAN, J. W. Microfabrication of ceramics by co-extrusion. Journal of the American Ceramic Society, v. 81, n. 1, p. 152-158, 1998.

HUANG, D. J.; DING, H. J.; CHEN, W. Q. Piezoelasticity solutions for functionally graded piezoelectric beams. Smart Materials 65 Structures, Iop Publishing Ltd, v. 16, n. 3, p. 687-695, jun. 2007.

HUSSEIN, M. I.; HAMZA, K.; HUlBERT, G. M.; SCOTT, R. A.; SAITOU, K. Multiobjective evolutionary optimization of periodic layered materials for desired wave dispersion characteristics. Structural and Multidisciplinary Optimization, v. 31, n. 1, p. 60-75, 2006.

IKEDA, T. Fundamentals of Piezoelectricity. [S.l.]: Oxford University Press, 1996.

IM, J.; KIM, S.; ROH, Y. R. Design and evaluation of piezoelectric bimorphs combined with multilayer actuator using fem. Journal of the Korean Physical Society, v. 32, p. S1251-S1253, fev. 1998.

JAMES, R.; WOODLEY, S. M.; DYER, C. M.; HUMPHREY, V. F. Sonic bands, bandgaps, and defect states in layered structures - theory and experiment. Journal of the Acoustical Society of America, v. 97, n. 4, p. 2041-2047, abr. 1995.

JAYACHANDRAN, K. P.; GUEDES, J. M.; RODRIGUES, H. C. Homogenized electromechanical properties of crystalline and ceramic relaxor ferroelectric $0.58 \mathrm{pb}($ mg1/3nb2/3)o-3-0.42pbtio(3). Smart Materials and Structures, v. 16, p. 1534-1541, 2007.

JAYACHANDRAN, K. P.; GUEDES, J. M.; RODRIGUES, H. C. Piezoelectricity enhancement in ferroelectric ceramics due to orientation. Applied Physics Letters, v. 92, n. 23, p. 232901, jun. 2008.

JOG, C. S.; HABER, R. B. Stability of finite element models for distributed-parameter optimization and topology design. Computer Methods in Applied Mechanics and Engineering, v. 130, n. 3-4, p. 203-226, 1996.

JOG, C. S.; HABER, R. B.; BENDSOE, M. P. Topology design with optimized, self-adaptive materials. International Journal For Numerical Methods In Engineering, John Wiley \& Sons Ltd, v. 37, n. 8, p. 1323-1350, abr. 1994.

JONES, R. Mechanics of Composite Materials. [S.l.]: CRC Press, 1998.

KANG, Z.; TONG, L. Integrated optimization of material layout and control voltage for piezoelectric laminated plates. Journal of Intelligent Material Systems and Structures, v. 19, n. 8, p. 889-904, ago. 2008.

KANG, Z.; TONG, L. Topology optimization-based distribution design of actuation voltage in static shape control of plates. Computers and Structures, v. 86, n. 19-20, p. 1885-1893, out. 2008. 
KAWAI, M. JR East Starts Test of Floor Able to Generate Power with Weight Applied by Passengers Going Through Ticket Gate, disponivel em: http: //techon.nikkeibp.co.jp/english/NEW $S_{E} N / 20061017 / 122345 / .2006$.

KEE, C. S.; KIM, J. E.; PARK, H. Y.; CHANG, K. J.; LIM, H. Essential role of impedance in the formation of acoustic band gaps. Journal of Applied Physics, v. 87, n. 4 , p. $1593-1596$, fev. 2000.

KIEBACK, B.; NEUBRAND, A.; RIEDEL, H. Processing techniques for functionally graded materials. Materials Science and Engineering, v. 362, n. 1-2, p. 81-105, dez. 2003 .

KIM, J. E.; KIM, D. S.; MA, P. S.; KIM, Y. Y. Multi-physics interpolation for the topology optimization of piezoelectric systems. Computer Methods in Applied Mechanics and Engineering, v. 199, n. 49-52, p. 3153-3168, 2010.

KIM, J. H.; PAULINO, G. H. Isoparametric graded finite elements for nonhomogeneous isotropic and orthotropic materials. ASME Journal of Applied Mechanics, v. 69, n. 4, p. 502-514, 2002.

KIYONO, C. Y.; SILVA, E. C. N.; REDDY, J. N. Design of laminated piezocomposite shell transducers with arbitrary fiber orientation using topology optimization approach. International Journal For Numerical Methods In Engineering, v. 90, n. 12, p. 1452-1484, jun. 2012.

KOGL, M.; SILVA, E. C. N. Topology optimization of smart structures: design of piezoelectric plate and shell actuators. Smart Materials and Structures, v. 14, n. 2, p. 387-399, 2005.

KOIZUMI, M. FGM activities in Japan. Composites Part B - Engineering, v. 28, n. 1-2, p. 1-4, 1997.

KRASZEWSKI, R. J.; SIGELMANN, R. A.; HERBERTZ, J. Electrical multiport transfer-functions and efficiency in layered media of piezoelectric and or nonpiezoelectric materials. Journal of the Acoustical Society of America, v. 90, n. 5, p. 2769-2774, 1991.

KRASZEWSKI, R. J.; SIGELMANN, R. A.; HERBERTZ, J.; LEE, M. A chain matrix model for an acoustically coupled, lead zirconate titanate core, electrical transformer. Journal of the Acoustical Society of America, Amer Inst Physics, v. 90, n. 5, p. 2763-2768, nov. 1991.

KRAUTKRAMER J.; KRAUTKRAMER, H. Ultrasonic Testing of Materials. Second Edition. New York: Springer-Verlag, 1977.

KUSHWAHA, M. S.; HALEVI, P.; DOBRZYNSKI, L.; DJAFARIROUHANI, B. Acoustic band-structure of periodic elastic composites. Physical Review Letters, v. 71, n. 13, p. 2022-2025, 1993.

KUSHWAHA, M. S.; HALEVI, P.; MARTINEZ, G.; DOBRZYNSKI, L.; DJAFARIROUHANI, B. Theory of acoustic band-structure of periodic elastic composites. Physical Review B, v. 49, n. 4, p. 2313-2322, jan. 1994.

LAI, Y.; ZHANG, Z. Q. Large band gaps in elastic phononic crystals with air inclusions. Applied Physics Letters, v. 83, n. 19, p. 3900-3902, nov. 2003. 
LAMBERTI, N.; ESPINOSA, F. R. M. de; IUlA, A.; CAROTENUTO, R. Two-dimensional modelling of multifrequency piezocomposites. Ultrasonics, v. 37, n. 8, p. $577-583$, jan. 2000.

LANGLET, P.; HLADKYHENNION, A. C.; DECARPIGNY, J. N. Analysis of the propagation of plane acoustic-waves in passive periodic materials using the finite-element method. Journal of the Acoustical Society of America, v. 98, n. 5, p. 2792-2800, nov. 1995.

LARSEN, U. D.; SIGMUND, O.; BOUWSTRA, S. Design and fabrication of compliant micromechanisms and structureswith negative poissons ratio. Journal of Microelectromechanical Systems, v. 6, n. 2, p. 99-106, 1997.

LEE, S.; YOUN, B. D. A new piezoelectric energy harvesting design concept: Multimodal energy harvesting skin. IEEE Transactions on Ultrasonics, Ferroelectrics and Frequency Control, v. 58, n. 3, p. 629-645, mar. 2011.

LENGLET, E.; HLADKY-HENNION, A. C.; DEBUS, J. C. Numerical homogenization techniques applied to piezoelectric composites. Journal of the Acoustical Society of America, v. 113, n. 2, p. 826-833, fev. 2003.

LERCH, R. Simulation of piezoelectric devices by 2-dimensional and 3-dimensional finite-elements. IEEE Transactions on Ultrasonics, Ferroelectrics and Frequency Control, v. 37, n. 3, p. 233-247, 1990.

LI, Q.; STEVEN, G. P.; XIE, Y. M. On equivalence between stress criterion and stiffness criterion in evolutionary structural optimisation. Structural Optimization, Springer Verlag, v. 18, n. 1, p. 67-73, ago. 1999.

LI, Q.; STEVEN, G. P.; XIE, Y. M. Evolutionary thickness design with stiffness maximization and stress minimization criteria. International Journal For Numerical Methods In Engineering, John Wiley \& Sons Ltd, v. 52, n. 9, p. 979-995, nov. 2001.

LI, S.; LISSENDEN, C. J. Piezoelectric fiber composite strip transducer design considerations for generating lamb waves. Journal of Intelligent Material Systems and Structures, v. 22, n. 12, p. 1345-1358, ago. 2011.

LIPTON, R.; NORTHRUP, J. Optimal bounds on the inplane shear moduli for orthotropic elasticcomposites. SIAM Journal on Applied Mathematics, v. 54, n. 2, p. 428-442, 1994.

LLOYD, S. P. Least-squares quantization in PCM. IEEE Transactions on Information Theory, v. 28, n. 2, p. 129-137, 1982.

MATSUI, K.; TERADA, K. Continuous approximation of material distribution for topology optimization. International Journal for Numerical Methods in Engineering, v. 59, n. 14, p. 1925-1944, 2004.

MAZUMDER, J.; DUTTA, D.; KIKUCHI, N.; GHOSH, A. Closed loop direct metal deposition: Art to part. Optics and Lasers in Engineering, v. 34, n. 4-6, p. 397-414, 2000 .

MAZUMDER, J.; SCHIFFERER, A.; CHOI, J. Direct materials deposition: designed macro and microstructure. Materials Research Innovations, v. 3, n. 3, p. 118-131, 1999. 
MENCIASSI, A.; EISINBERG, A.; CARROZZA, M. C.; DARIO, P. Force sensing microinstrument for measuring tissue properties and pulse in microsurgery. IEEE-ASME Transactions on Mechatronics, v. 8, n. 1, p. 10-17, 2003.

MILTON, G. W.; CHERKAEV, A. V. Which elasticity tensors are realizable? Journal of Engineering Materials and Technology-Transactions of The Asme, v. 117, n. 4, p. 483-493, out. 1995.

MOGAMI, K.; NISHIWAKI, S.; IZUI, K.; YOSHIMURA, M.; NOMURA, T. Structural optimization for the design of band-gap structures using discrete structural elements. In: 11th AIAA/ISSMO Multidisciplinary Analysis and Optimization Conference. [S.l.: s.n.], 2006.

NAILLON, M.; COURSANT, R. H.; BESNIER, F. Analysis of piezoelectric structures by a finite-element method. Acta Electronica, v. 25, n. 4, p. 341-362, 1983.

NAKASONE, P. H.; SILVA, E. C. N. Dynamic design of piezoelectric laminated sensors and actuators using topology optimization. Journal of Intelligent Material Systems and Structures, v. 21, n. 16, p. 1627-1652, nov. 2010.

NEWNHAM, R. E.; SKInNER, D. P.; CROSS, L. E. Connectivity and piezoelectric-pyroelectric composites. Materials Research Bulletin, Pergamon-Elsevier Science Ltd, v. 13, n. 5, p. 525-536, 1978.

NGUYEN, T. H.; PAUlinO, G. H.; SONG, J.; LE, C. H. A computational paradigm for multiresolution topology optimization (MTOP). Structural and Multidisciplinary Optimization, v. 41, n. 4, p. 525-539, 2010.

NI, A. Q.; ZHU, Y. W.; WANG, J. H. Determination of the micro stress field in composite by homogenization method. Journal of Wuhan University of Technology-materials Science Edition, v. 21, n. 1, p. 114-117, mar. 2006.

NOVOTnY, A. A.; FEIJOO, R. A.; TAROCO, E.; PADRA, C. Topological sensitivity analysis. Computer Methods In Applied Mechanics and Engineering, Elsevier Science Sa, v. 192, n. 7-8, p. 803-829, 2003.

ODEN, J. T.; KIKUCHI, N.; SONG, Y. J. Penalty-finite element methods for the analysis of stokesian flows. Computer Methods In Applied Mechanics and Engineering, Elsevier Science Sa Lausanne, v. 31, n. 3, p. 297-329, 1982.

OTERO, J. A.; CASTILlERO, J. B.; RAMOS, R. R. Homogenization of heterogeneous piezoelectric medium. Mechanics Research Communications, v. 24, n. 1, p. 75-84, 1997.

PEREZ, N.; NEGREIRA, C.; GOMEZ, H.; BELLO, C. Piezocomposite array transducers optimization by breaking periodicity. 1999 IEEE Ultrasonics Symposium Proceedings, Vols 1 and 2, p. 1081-1084, 1999.

PHANI, A. S.; WOODHOUSE, J.; FLECK, N. A. Wave propagation in two-dimensional periodic lattices. Journal of the Acoustical Society of America, v. 119, n. 4, p. 1995-2005, 2006.

PIEFORT, V. Finite element modelling of piezoelectric active structures. Tese (Doutorado) - ULB - Université Libre de Bruxelles, Brussels - Belgium, 2001. 
PRIYA, S. Advances in energy harvesting using low profile piezoelectric transducers. Journal of Electroceramics, v. 19, n. 1, p. 165-182, set. 2007.

QI, H.; KIKUCHI, N.; MAZUMDER, J. Interface study and boundary smoothing on designed composite material microstructures for manufacturing purposes. Structural and Multidisciplinary Optimization, v. 26, n. 5, p. 326-332, 2004.

QI, J.; HALLORAN, J. W. Negative thermal expansion artificial material from iron-nickel alloys by oxide co-extrusion with reductive sintering. Journal of Materials Science, v. 39, n. 13, p. 4113-4118, 2004.

QIU, J. H.; ZHU, K. J.; JI, H. L. Fabrication and performance of high temperature style functionally graded piezoelectric bending actuators. Modern Physics Letters B, v. 23, n. 3, p. 433-436, 2009.

RAHMATALLA, S. F.; SWAN, C. C. A Q4/Q4 continuum structural topology optimization implementation. Structural and Multidisciplinary Optimization, v. 27, n. 1-2, p. 130-135, 2004.

REILlY, C. J.; HALlORAN, J. W.; SILVA, E. C. N. Piezoelectric hydrophones from optimal designs, part i: fabrication. Journal of Materials Science, v. 42, n. 13, p. 4824-4831, 2007.

REILlY, C. J.; HALlORAN, J. W.; SILVA, E. C. N.; ESPINOSA, F. M. D. Piezoelectric hydrophones from optimal design, ii: properties. Journal of Materials Science, v. 42, n. 13, p. 4810-4814, 2007.

REITER, T.; DVORAK, G. J.; TVERGAARD, V. Micromechanical models for graded composite materials. Journal of the Mechanics and Physics of Solids, v. 45, n. 8, p. 1281-1302, 1997.

REYNAERTS, D.; PEIRS, J.; BRUSSEL, H. V. A mechatronic approach to microsystem design. IEEE-ASME Transactions on Mechatronics, v. 3, n. 1, p. 24-33, 1998.

ROHAN, E.; MIARA, B. Sensitivity analysis of acoustic wave propagation in strongly heterogeneous piezoelectric composite. In: . [S.l.: s.n.], 2006.

ROHAN, E.; MIARA, B.; SEIFRT, F. Numerical simulation of acoustic band gaps in homogenized elastic composites. International Journal of Engineering Science, v. 47, n. 4, p. 573-594, 2009.

ROZVANY, G. I. N. Optimal layout theory - analytical solutions for elastic structures with several deflection constraints and load conditions. Structural Optimization, Springer Verlag, v. 4, n. 3-4, p. 247-249, out. 1992.

ROZVANY, G. I. N. Aims, scope, methods, history and unified terminology of computer-aided topology optimization in structural mechanics. Structural and Multidisciplinary Optimization, v. 21, n. 2, p. 90-108, 2001.

RUBIO, W. M.; BUIOCHI, F.; ADAMOWSKI, J. C.; SILVA, E. C. N. Modeling of functionally graded piezoelectric ultrasonic transducers. Ultrasonics, Elsevier Science Bv, v. 49, n. 4-5, p. 484-494, maio 2009. 
RUBIO, W. M.; SILVA, E. C. N.; PAUlinO, G. H. Toward optimal design of piezoelectric transducers based on multifunctional and smoothly graded hybrid material systems. Journal of Intelligent Material Systems and Structures, v. 20, n. 14, p. 1725-1746, 2009.

RUPP, C. J. Topology Optimization for Wave Propagation and Vibration Phenomena in Elastic and Piezoelectric. Tese (Doutorado) - University of Colorado, Department of Mechanical Engineering, 2009.

RUPP, C. J.; EVGRAFOV, A.; MAUTE, K.; DUNN, M. L. Design of phononic materials/structures for surface wave devices using topology optimization. Structural and Multidisciplinary Optimization, v. 34, n. 2, p. 111-121, 2007.

RUPP, C. J.; EVGRAFOV, A.; MAUTE, K.; DUNN, M. L. Design of piezoelectric energy harvesting systems: A topology optimization approach based on multilayer plates and shells. Journal of Intelligent Material Systems and Structures, v. 20, n. 16, p. 1923-1939, 2009.

RUZZENE, M.; BAZ, A. Attenuation and localization of wave propagation in periodic rods using shape memory inserts. Smart Materials and Structures, v. 9, n. 6, p. 805-816, dez. 2000.

SAFARI, A. Development of piezoelectric composites for transducers. Journal de Physique III, v. 4, n. 7, p. 1129-1149, 1994.

SANCHEZ-PALENCIA, E. Non-Homogeneous Media and Vibration Theory. [S.l.]: Lectures Notes in Physics 127, Springer, Berlin, 1980.

SEELEY, C. E.; CHATTOPADHYAY, A.; BREI, D. Development of a polymeric piezoelectric c-block actuator using hybrid optimization technique. AIAA Journal, v. 34, n. 1, p. 123-128, jan. 1996.

SEO, Y.-D.; KIM, H.-J.; YOUN, S.-K. Isogeometric topology optimization using trimmed spline surfaces. Computer Methods In Applied Mechanics and Engineering, v. 199, n. 49-52, p. 3270-3296, 2010.

SHMUlEVICH, I.; GALILI, N.; HOWARTH, M. S. Nondestructive dynamic testing of apples for firmness evaluation. Postharvest Biology and Technology, v. 29, n. 3, p. 287-299, 2003.

SIGALAS, M. M. Defect states of acoustic waves in a two-dimensional lattice of solid cylinders. Journal of Applied Physics, v. 84, n. 6, p. 3026-3030, 1998.

SIGMUND, O. On the design of compliant mechanisms using topology optimization. Mechanics of Structures and Machines, v. 25, p. 495-526, 1997.

SIGMUND, O. A new class of extremal composites. Journal of the Mechanics and Physics of Solids, Pergamon-Elsevier Science Ltd, v. 48, n. 2, p. 397-428, fev. 2000.

SIGMUND, O. Topology optimization: a tool for the tailoring of structures and materials. Philosophical Transactions of the Royal Society A-Mathematical Physical and Engineering Sciences, Royal Soc London, v. 358, n. 1765, p. 211-227, jan. 2000.

SIGMUND, O. Design of multiphysics actuators using topology optimization - part ii: Two-material structures. Computer Methods in Applied Mechanics and Engineering, v. 190, p. 6605-6627, 2001. 
SIGMUND, O. Design of multiphysics actuators using topology optimization - part i: One-material structures. Computer Methods in Applied Mechanics and Engineering, v. 190, p. 6577-6604, 2001.

SIGMUND, O. Morphology-based black and white filters for topology optimization. Structural and Multidisciplinary Optimization, v. 33, n. 4-5, p. 401-424, abr. 2007.

SIGMUND, O.; JENSEN, J. S. Systematic design of phononic band-gap materials and structures by topology optimization. Philosophical Transactions of the Royal Society of London Series A-Mathematical Physical and Engineering Sciences, v. 361, n. 1806, p. 1001-1019, 2003.

SIGMUND, O.; PETERSSON, J. Numerical instabilities in topology optimization: A survey on procedures dealing with checkerboards, mesh-dependencies and local minima. Structural Optimization, v. 16, n. 1, p. 68-75, ago. 1998.

SIGMUND, O.; TORQUATO, S. Design of materials with extreme thermal expansion using a three-phase topology optimization method. Journal of the Mechanics and Physics of Solids, Pergamon-Elsevier Science Ltd, v. 45, n. 6, p. 1037-1067, jun. 1997.

SILVA, E. C. N.; CARBONARI, R. C.; PAUlinO, G. H. On graded elements for multiphysics applications. Communications in Numerical Methods in Engineering, v. 16, p. 2408-2428, 2007.

SILVA, E. C. N.; FONSECA, J. S. O.; ESPINOSA, F. M. de; CRUMM, A. T.; BRADY, G. A.; HALLORAN, J. W.; KIKUCHI, N. Design of piezocomposite materials and piezoelectric transducers using topology optimization - Part I. Archives of Computational Methods in Engineering, v. 6, n. 2, p. 117-182, 1999.

SILVA, E. C. N.; FONSECA, J. S. O.; KIKUCHI, N. Optimal design of piezoelectric microstructures. Computational Mechanics, v. 19, n. 5, p. 397-410, 1997.

SILVA, E. C. N.; KIKUCHI, N. Design of piezocomposite materials and piezoelectric transducers using topology optimization - Part III. Archives of Computational Methods in Engineering, v. 6, n. 4, p. 305-329, 1999.

SILVA, E. C. N.; NISHIWAKI, S.; FONSECA, J. S. O.; KIKUCHI, N. Optimization methods applied to material and flextensional actuator design using the homogenization method. Computer Methods in Applied Mechanics and Engineering, v. 172, n. 1-4, p. 241-271, abr. 1999.

SILVA, E. C. N.; NISHIWAKI, S.; KIKUCHI, N. Topology optimization design of flextensional actuators. IEEE Transactions on Ultrasonics Ferroelectrics and Frequency Control, v. 47, n. 3, p. 657-671, 2000.

SMITH, W. The role of piezocomposites in ultrasonic transducers. Ultrasonics Symposium, 1989. Proceedings., IEEE 1989, Montreal, Canada, v. 2, p. 755-766, 1989.

SMITH, W. A. Limits to the enhancement of piezoelectric transducers achievable by materials engineering. Proceedings of IEEE Ultrasonics Symposium, p. 697-702, 1992.

SMITH, W. A. Modeling 1-3 composite piezoelectrics - hydrostatic response. IEEE Transactions on Ultrasonics Ferroelectrics and Frequency Control, v. 40, n. 1, p. 41-49, 1993. 
SMITH, W. A.; AULD, B. A. Modeling 1-3 composite piezoelectrics: Thickness-mode oscillations. IEEE Trans. on Ultrasonics, Ferroelectrics, and Frequency Control, v. 38, p. 40-47, 1991.

SMITS, J. G.; DALKE, S. I.; COONEY, T. K. The constituent equations of piezoelectric bimorphs. Sensors and Actuators A-physical, v. 28, n. 1, p. 41-61, jun. 1991.

STEGMANN, J.; LUND, E. Discrete material optimization of general composite shell structures. International Journal for Numerical Methods in Engineering, v. 62, n. 14, p. 2009-2027, 2005.

STROMBERG, L. L.; BEGHINI, A.; BAKER, W. F.; PAUlinO, G. H. Application of layout and topology optimization using pattern gradation for the conceptual design of buildings. Structural and Multidisciplinary Optimization, v. 43, n. 2, p. 165-180, fev. 2011.

SUKUMAR, N.; TABARRAEI, A. Conforming polygonal finite elements. International Journal for Numerical Methods in Engineering, v. 61, n. 12, p. 2045-2066, 2004.

SUZUKI, K.; KIKUCHI, N. A homogenization method for shape and topology optimization. Computer Methods in Applied Mechanics and Engineering, v. 93, p. 291-318, 1991.

SVANBERG, K. The method of moving asymptotes - a new method for structural optimization. International Journal for Numerical Methods in Engineering, v. 24, p. 359-373, 1987.

TAKEZAWA, A.; NISHIWAKI, S.; KITAMURA, M. Shape and topology optimization based on the phase field method and sensitivity analysis. Journal of Computational Physics, Academic Press Inc Elsevier Science, v. 229, n. 7, p. 2697-2718, abr. 2010.

TALiSCHI, C.; PAUlinO, G. H.; PEREIRA, A.; MENEZES, I. F. M. Polygonal finite elements for topology optimization: A unifying paradigm. International Journal for Numerical Methods in Engineering, p. 28, 2009.

TANAKA, Y.; TOMOYASU, Y.; TAMURA, S. Band structure of acoustic waves in phononic lattices: Two-dimensional composites with large acoustic mismatch. Physical Review B, v. 62, n. 11, p. 7387-7392, 2000.

TELEGA, J. J. Piezoelectricity and homogenization: Application to biomechanics. Continuum Models and Discrete Systems, v. 2, p. 220-230, 1990.

TIEN, C. M. T.; GOO, N. S. Use of a piezocomposite generating element in energy harvesting. Journal of Intelligent Material Systems and Structures, v. 21, n. 14, p. 1427-1436, set. 2010.

TIERSTEN, H. Hamilton's principle for linear piezoelectric media. Proceedings of the IEEE, v. 55, n. 8, p. 1523-1524, 1967.

TONGELE, T. N.; CHEN, T. Control of longitudinal wave propagation in conical periodic structures. Journal of Vibration and Control, v. 10, n. 12, p. 1795-1811, dez. 2004.

TORRENT, D.; SANCHEZ-DEHESA, J. Acoustic cloaking in two dimensions: a feasible approach. New Journal of Physics, v. 10, p. 063015, 2008. 
TRINDADE, M. A.; BENJEDDOU, A. Finite element homogenization technique for the characterization of d(15) shear piezoelectric macro-fibre composites. Smart Materials $\&$ Structures, Iop Publishing Ltd, v. 20, n. 7, p. 075012, jul. 2011.

TURBE, N.; MAUGIN, G. A. On the linear piezoelectricity of composite-materials. Mathematical Methods in The Applied Sciences, v. 14, n. 6, p. 403-412, 1991.

VASSEUR, J. O.; DEYMIER, P. A.; FRANTZISKONIS, G.; HONG, G.; DJAFARI-ROUHANI, B. Experimental evidence for the existence of absolute acoustic band gaps in two-dimensional periodic composite media. Journal of Physics-Condensed Matter, v. 10, n. 27, p. 6051-6064, jul. 1998.

VATANABE, S.; PAULINO, G.; SILVA, E. Design of functionally graded piezocomposite materials using topology optimization. In: $13^{\text {th }}$ AIAA/ISSMO Multidisciplinary Analysis Optimization Conference. FortWorth, TX, EUA: [s.n.], 2010.

VATANABE, S.; PAULINO, G.; SILVA, E. Design of functionally graded piezocomposite materials using topology optimization with polygonal mesh. In: ASME 2010 International Mechanical Engineering Congress \& Exposition. Vancouver, Canada: [s.n.], 2010.

VATANABE, S.; PAULINO, G.; SILVA, E. A multiscale approach to design of functionally graded piezocomposite materials for energy harvesting. In: $10^{\text {th }}$ WCCM World Congress on Computational Mechanics. Sao Paulo, SP: [s.n.], 2012.

VATANABE, S.; SILVA, E. Application of topology optimization using pattern gradation for piezoelectric energy harvesting. In: $11^{\text {th }}$ U.S. National Congress on Computational Mechanics. Minneapolis, MN, EUA: [s.n.], 2011.

VATANABE, S.; SILVA, E. Design of phononic band gaps in functionally graded piezocomposite materials by using topology optimization. In: 2011 Smart Structures/NDE. San Diego, CA, EUA: [s.n.], 2011.

VATANABE, S.; SILVA, E. The influence of objective function formulation in the design of dynamic piezocomposite energy harvesting devices using topology optimization. In: $10^{\text {th }}$ WCCM - World Congress on Computational Mechanics. Sao Paulo, SP: [s.n.], 2012.

VATANABE, S. L.; PAULINO, G. H.; SILVA, E. C. N. Influence of pattern gradation on the design of piezocomposite energy harvesting devices using topology optimization. Composites Part B-engineering, v. 43, n. 6, p. 2646-2654, set. 2012.

VILLENEUVE, P. R.; PICHE, M. Photonic band-gaps of transverse-electric modes in 2-dimensionally periodic media. Journal of the Optical Society of America A-optics Image Science and Vision, v. 8, n. 8, p. 1296-1305, ago. 1991.

WANG, B. L.; NODA, N. Design of a smart functionally graded thermopiezoelectric composite structure. Smart Materials and Structures, v. 10, n. 2, p. 189-193, 2001.

WANG, S. Y.; WANG, M. Y. Radial basis functions and level set method for structural topology optimization. International Journal For Numerical Methods In Engineering, Wiley-blackwell, v. 65, n. 12, p. 2060-2090, mar. 2006.

WANG, Y. Z.; LI, F. M.; HUANG, W. H.; WANG, Y. S. Effects of inclusion shapes on the band gaps in two-dimensional piezoelectric phononic crystals. Journal of Physics-Condensed Matter, v. 19, n. 49, 2007. 
WANG, Y. Z.; LI, F. M.; KISHIMOTO, K.; WANG, Y. S.; HUANG, W. H. Wave band gaps in three-dimensional periodic piezoelectric structures. Mechanics Research Communications, v. 36, n. 4, p. 461-468, 2009.

WEI, R. J.; WU, B.; HE, C. F.; ZHAO, H. Y. Phononic band structure in a two-dimensional hybrid triangular graphite lattice. Physica B-Condensed Matter, v. 404, n. 20, p. 3795-3798, nov. 2009.

WING, Z. N.; HALLORAN, J. W. Dry powder deposition and compaction for functionally graded ceramics. Journal of the American Ceramic Society, v. 89, n. 11, p. 3406-3412, 2006.

WU, H. D.; LIN, J. M.; GAO, H.; SHUI, Y. G.; XUE, Q. Optimization of composite transducer designing in high frequency applications. IEEE Transactions on Ultrasonics, Ferroelectrics and Frequency Control, v. 47, n. 6, p. 1610-1614, 2000.

WU, L. Y.; WU, M. L.; CHEN, L. W. The narrow pass band filter of tunable 1d phononic crystals with a dielectric elastomer layer. Smart Materials and Structures, v. 18, n. 1, p. 015011, jan. 2009.

WU, M. L.; WU, L. Y.; YANG, W. P.; CHEN, L. W. Elastic wave band gaps of one-dimensional phononic crystals with functionally graded materials. Smart Materials and Structures, Iop Publishing Ltd, v. 18, n. 11, p. 115013, nov. 2009.

YAMADA, K.; OHKUBO, A.; NAKAMURA, K. Functional grading of a piezoelectric 1-3 composite for ultrasound transducers with enhanced axial and lateral resolution. 2001 IEEE Ultrasonics Symposium Proceedings, Vols 1 and 2, Ieee, p. 1031-1034, 2001.

YAS, M. H.; JODAEI, A.; IRANDOUST, S.; AGHDAM, M. N. Three-dimensional free vibration analysis of functionally graded piezoelectric annular plates on elastic foundations. Meccanica, v. 47, n. 6, p. 1401-1423, ago. 2012.

YEH, J. Y.; CHEN, L. W. Wave propagations of a periodic sandwich beam by fem and the transfer matrix method. Composite Structures, v. 73, n. 1, p. 53-60, maio 2006.

YIN, H. M.; PAUlinO, G. H.; BUTTLAR, W. G.; SUN, L. Z. Micromechanics-based thermoelastic model for functionally graded particulate materials with particle interactions. Journal of the Mechanics and Physics of Solids, v. 55, n. 1, p. 132-160, 2007.

YIP, M.; MOHLE, J.; BOLANDER, J. E. Automated modeling of three-dimensional structural components using irregular lattices. Computer-aided Civil and Infrastructure Engineering, v. 20, n. 6, p. 393-407, nov. 2005.

YOO, J.; KIKUCHI, N. Topology optimization in magnetic fields using homogenization design method. International Journal for Numerical Methods in Engineering, v. 48, p. 1463-1479, 2000.

ZHANG, X.; LIU, Z. Y.; LIU, Y. Y.; WU, F. G. Elastic wave band gaps for three-dimensional phononic crystals with two structural units. Physics Letters A, v. 313, n. 5-6, p. 455-460, 2003.

ZHENG, B.; CHANG, C. J.; GEA, H. C. Topology optimization of energy harvesting devices using piezoelectric materials. Structural and Multidisciplinary Optimization, v. 38, n. 1, p. 17-23, 2009. 
ZHU, M.; WORTHINGTON, E.; NJUGUNA, J. Analyses of power output of piezoelectric energy-harvesting devices directly connected to a load resistor using a coupled piezoelectric-circuit finite element method. IEEE Transactions on Ultrasonics, Ferroelectrics and Frequency Control, v. 56, n. 7, p. 1309-1318, jul. 2009.

ZOU, X. Y.; CHEN, Q.; LIANG, B.; CHENG, J. C. Control of the elastic wave bandgaps in two-dimensional piezoelectric periodic structures. Smart Materials and Structures, v. 17, n. $1,2008$.

ZUH, X.; MENG, Z. Operational principle, fabrication and displacement characteristics of a functionally gradient piezoelectric ceramic actuator. Sensor and Actuators A, v. 48, p. 169-176, 1995. 


\section{Apêndice A - Materiais Piezelétricos}

Materiais piezelétricos tem a propriedade de converter energia elétrica (potência e cargas elétricas) em energia mecânica (tensão e deformação mecânicas) e vice-versa. Essa propriedade é observada quando o material piezelétrico se contrai e se expande de acordo com a tensão elétrica aplicada em seus terminais e vice-versa, gerando deformação no sentido longitudinal e transversal de acordo com a excitação aplicada, como mostra a Fig. A.1. Exemplos de materiais piezelétricos incluem quartzo, cerâmica (PZT) e polímeros (PVDF). No campo da Engenharia a utilização mais comum do efeito piezelétrico é em transdutores piezelétricos, cujas aplicações vão desde sensores e atuadores na micro (MEMS) e macroescala, até em acústica, tais como transdutores de ultrassom, hidrofones, sonares, detecção de imagens para aplicações médicas e testes não-destrutivos, entre outros (SMITH, 1989; REYNAERTS; PEIRS; BRUSSEL, 1998; SILVA et al., 1999; PEREZ et al., 1999; WU et al., 2000; MENCIASSI et al., 2003; SHMULEVICH; GALILI; HOWARTH, 2003). Um estudo de diversas aplicações de materiais piezelétricos é apresentado por Ikeda (IKEDA, 1996).

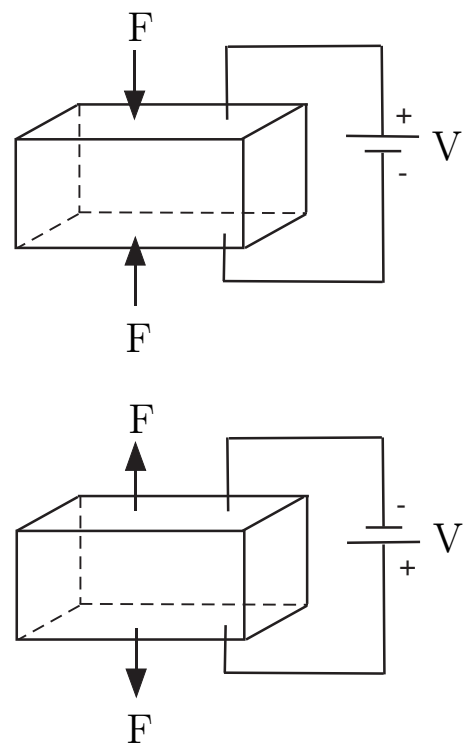

(c)
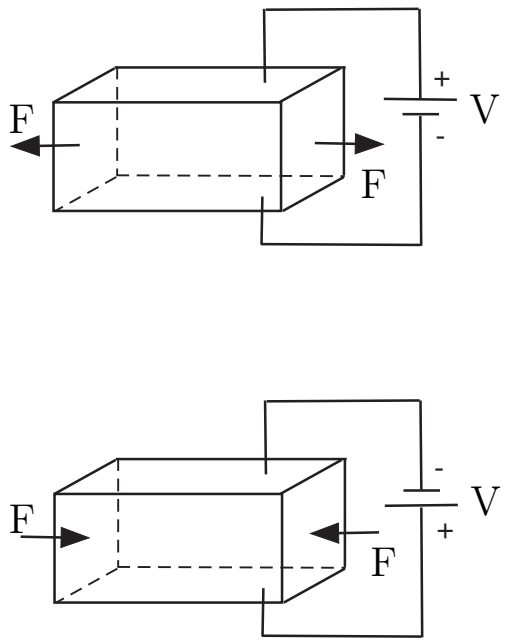

(d)

Figura A.1: Efeitos (a) longitudinais e (b) transversais das cerâmicas piezelétricas.

Neste trabalho são considerados materiais piezelétricos que, embora apresentem um comportamento não linear, assume-se que na faixa de operação tratada nesse estudo 
a resposta seja linear à variações no campo e deslocamento elétricos ou tensões e deformações mecânicas. Variações de temperatura e de campo magnético são consideradas desprezíveis. Estas hipóteses são compatíveis com cerâmicas piezelétricas, polímeros e compósitos. Portanto, o comportamento de um meio piezelétrico pode ser descrito pelas seguintes equações constitutivas (IKEDA, 1996):

$$
\begin{aligned}
T_{i j} & =c_{i j k l}^{E} S_{k l}-e_{k i j} E_{k} \\
D_{i} & =e_{i k l} S_{k l}+\varepsilon_{i k}^{S} E_{k}
\end{aligned}
$$

onde $c_{i j k l}^{E}$ é um tensor elástico de quarta ordem sob condições de contorno de curto-circuito, $\varepsilon_{i k}^{S}$ é um tensor dielétrico de corpo livre de segunda ordem e $e_{k i j}$ é um tensor de deformação piezelétrico de terceira ordem.

Devido às simetrias dos tensores $T_{i j}, S_{i j}, c_{i j k l}$ e $\varepsilon_{i j}$ a Eq. (A.1) pode ser reescrita na notação compacta (ANSI/IEEE, 1984):

$$
\begin{aligned}
& \mathbf{T}=\mathbf{c}^{E} \mathbf{S}-\mathbf{e E} \\
& \mathbf{D}=\mathbf{e}^{t} \mathbf{S}+\varepsilon^{S} \mathbf{E}
\end{aligned}
$$

onde:

$$
\begin{aligned}
& \mathbf{T}=\text { tensor de tensões mecânicas; } \\
& \mathbf{D}=\text { vetor deslocamento elétrico; } \\
& \boldsymbol{c}^{\mathbf{E}}=\text { tensor de rigidez elástica obtido com campo elétrico constante; } \\
& \mathbf{S}=\text { tensor de deformações mecânicas; } \\
& \mathbf{e}=\text { tensor de coeficientes piezelétricos de tensão mecânica; } \\
& \boldsymbol{\varepsilon}^{S}=\text { tensor dielétrico medido com deformação constante; } \\
& \mathbf{E}=\text { vetor campo elétrico. }
\end{aligned}
$$

O sobrescrito "t" indica a matriz transposta.

No caso de materiais piezocompósitos, se o comprimento de onda do campo aplicado for muito maior que o tamanho da célula unitária (ou da microestrutura), o material compósito pode ser modelado como um meio homogêneo. neste caso, seu comportamento pode ser caracterizado pela Eq. (A.2), substituindo os devidos termos pelas propriedades efetivas (ou homogeneizadas) do compósito nestas equações (SMITH, 1993). As propriedades efetivas podem ser calculadas utilizando o método de homogeneização apresentado na seção 2.1. Portanto, as equações constitutivas do material compósito considerando as propriedades homogeneizadas ficam: 


$$
\left\{\begin{array}{c}
\langle\mathbf{T}\rangle=\mathbf{c}_{H}^{E}\langle\mathbf{S}\rangle-\mathbf{e}_{H}\langle\mathbf{E}\rangle \\
\langle\mathbf{D}\rangle=\mathbf{e}_{H}^{t}\langle\mathbf{S}\rangle+\varepsilon_{H}^{S}\langle\mathbf{E}\rangle
\end{array}\right.
$$

As equações constitutivas também podem ser apresentadas pela forma alternativa:

$$
\left\{\begin{array}{l}
\langle\mathbf{S}\rangle=\mathbf{s}_{H}^{E}\langle\mathbf{T}\rangle+\mathbf{d}_{H}\langle\mathbf{E}\rangle \\
\langle\mathbf{D}\rangle=\mathbf{d}_{H}^{t}\langle\mathbf{T}\rangle+\varepsilon_{H}^{T}\langle\mathbf{E}\rangle
\end{array}\right.
$$

onde $\langle$.$\rangle indica quantidades médias volumétricas \frac{1}{|V|} \int_{V} . d V$ e o subscrito "H" refere-se às propriedades homogeneizadas. $\mathbf{s}_{H}^{E}$ é o tensor flexibilidade homogeneizado sob condições de curto-circuito, $\varepsilon_{H}^{T}$ é o tensor dielétrico homogeneizado de corpo engastado e $\mathbf{d}_{H}$ é o tensor de tensões piezelétrico homogeneizado.

Também é possível escrever a Eq. (A.3) na forma:

$$
\left\{\begin{array}{c}
\langle\mathbf{S}\rangle \\
\langle\mathbf{D}\rangle
\end{array}\right\}=\left[\begin{array}{cc}
\mathbf{s}_{H}^{E} & \mathbf{d}_{H} \\
\mathbf{d}_{H}^{t} & \varepsilon_{H}^{T}
\end{array}\right]\left\{\begin{array}{c}
\langle\mathbf{T}\rangle \\
\langle\mathbf{E}\rangle
\end{array}\right\}
$$

Neste caso, a matriz pode ser interpretada como um supertensor que contém propriedades elásticas, piezelétricas e dielétricas. O espaço de propriedades viáveis para materiais piezelétricos é obtido analisando a positividade deste tensor (SMITH, 1992). Modificando a topologia da célula unitária, é possível obter novas propriedades efetivas neste espaço (SILVA; FONSECA; KIKUCHI, 1997). O intervalo das propriedades efetivas que podem ser obtidas será determinado por limites que dependem das propriedades de cada material constituinte da microestrutura analisada. Pelo conhecimento do bolsista, estes limites não estão disponíveis na literatura para materiais piezelétricos. Entretanto, Gibiansky e Torquato (GIBIANSKY; TORQUATO, 1995) desenvolveram limites de propriedades elásticas e de condutividade de materiais compósitos feitos de dois materiais (não necessariamente isotrópicos) que podem ser estendidos para materiais piezelétricos.

As relações entre as propriedades das Eq. (A.3) e (A.4) são (ANSI/IEEE, 1984):

$$
\mathbf{s}_{H}^{E}=\left(\mathbf{c}_{H}^{E}\right)^{-1} \quad \varepsilon_{H}^{T}=\varepsilon_{H}^{S}+\mathbf{d}_{H}^{t}\left(\mathbf{s}_{H}^{E}\right)^{-1} \mathbf{d}_{H} \quad \mathbf{d}_{H}=\left(\mathbf{s}_{H}^{E}\right) \mathbf{e}_{H}
$$

Nas seções posteriores o subscrito "H" será omitido para as propriedades homogeneizadas para simplificar a notação. Como convenção, o eixo de polarização do material piezelétrico será considerado na direção 3 (ou z).

Na modelagem de microestruturas bidimensionais é considerado o estado plano de deformações. Portanto, assumindo que o modelo está no plano 1-3 (x-z) (y é a direção 
normal) e que o material piezelétrico utilizado nos piezocompósitos pertence à classe de simetria $6 \mathrm{~mm}$, as propriedades do estado plano de deformação correspondentes podem ser obtidas considerando $\varepsilon_{y}=0$ e $E_{y}=0$ na Eq. (A.2) e reescrevendo-as utilizando apenas os termos $\varepsilon_{i}, T_{i}, D_{i}$ e $E_{i}$, para $i=1$ ou 3 .

Para os materiais das classes hexagonais com anisotropia na direção $z$ são mostrados a seguir o tensor piezelétrico $(\mathbf{e})$, o tensor dielétrico $\left(\varepsilon^{S}\right)$ e o tensor de rigidez elástica $\left(\boldsymbol{c}^{\boldsymbol{E}}\right)$ na forma matricial (IKEDA, 1996):

$$
\begin{aligned}
\mathbf{e}= & {\left[\begin{array}{cccccc}
0 & 0 & 0 & 0 & e_{15} & 0 \\
0 & 0 & 0 & e_{15} & 0 & 0 \\
e_{31} & e_{31} & e_{33} & 0 & 0 & 0
\end{array}\right] } \\
\boldsymbol{\varepsilon}^{S}= & {\left[\begin{array}{ccc}
\varepsilon_{11} & 0 & 0 \\
0 & \varepsilon_{11} & 0 \\
0 & 0 & \varepsilon_{33}
\end{array}\right] } \\
\mathbf{c}^{E}= & {\left[\begin{array}{cccccc}
c_{11} & c_{12} & c_{13} & 0 & 0 & 0 \\
c_{12} & c_{22} & c_{13} & 0 & 0 & 0 \\
c_{13} & c_{13} & c_{33} & 0 & 0 & 0 \\
0 & 0 & 0 & c_{44} & 0 & 0 \\
0 & 0 & 0 & 0 & c_{55} & 0 \\
0 & 0 & 0 & 0 & 0 & c_{66}
\end{array}\right] } \\
S_{i j}= & \frac{1}{2}\left(\frac{\partial u_{i}}{\partial x_{j}}+\frac{\partial u_{j}}{\partial x_{i}}\right) \\
\mathbf{E}= & -\nabla \phi, \quad \text { onde } \phi \text { é o potencial elétrico. }
\end{aligned}
$$

O comportamento elástico de um meio piezelétrico é governado pela equação de Newton

$$
\operatorname{div} \mathbf{T}=\rho \partial^{2} \mathbf{u} / \partial t^{2}
$$

onde div é o operador divergente e $\rho$ é a densidade do meio piezelétrico, enquanto que o comportamento elétrico é descrito pela equação de Maxwell

$$
\operatorname{div} \mathbf{D}=0
$$

considerando que o meio piezelétrico está isolado eletricamente. Utilizando a Eq. A.1 de materiais piezelétricos juntamente com as Eqs. (A.12) e (A.13), obtém-se a equação dinâmica do sistema (LERCH, 1990): 


$$
\begin{aligned}
\frac{\partial}{\partial r}\left[\mathbf{c}^{E}(x, y) S-\mathbf{e}(x, y) E\right] & =-\rho(x, y) \omega^{2} \mathbf{u} \\
\frac{\partial}{\partial r}\left[\mathbf{e}^{T}(x, y) S+\boldsymbol{\varepsilon}^{S}(x, y) E\right] & =0
\end{aligned}
$$

onde $r$ é o vetor unitário no sistema de coordenadas cartesianas, u é o vetor deslocamento e $\omega$ é a frequência angular. 


\section{Apêndice B - Método de Elementos Finitos (MEF) para Materiais Piezelétricos}

Nesta seção, primeiramente é apresentada a formulação variacional para uma estrutura piezelétrica, juntamente com a resolução do problema piezelétrico utilizando o princípio de Hamilton $^{1}$ (TIERSTEN, 1967). Posteriormente, é desenvolvida a formulação de elementos finitos para problemas piezelétricos.

\section{B.1 Formulação Variacional para Problemas Piezelétricos}

Segundo esse princípio, os deslocamentos e potenciais elétricos que realmente ocorrem no problema são aqueles que satisfazem a seguinte equação (TIERSTEN, 1967):

$$
\delta \int_{t_{1}}^{t_{2}} L d t+\int_{t_{1}}^{t_{2}} \delta W d t=0
$$

onde $L$ é o Lagrangeano e $W$ é o trabalho externo realizado pelas forças mecânicas e elétricas, e $\delta$ representa o variacional. O Lagrangeano é dado por (PIEFORT, 2001):

$$
L=\int_{\Omega}\left(\frac{1}{2} \rho \dot{\mathbf{u}}^{T} \dot{\mathbf{u}}-H\right) d \Omega
$$

onde $H$ é a entalpia elétrica e u é o vetor de deslocamentos. A integração da Eq. é realizada sobre um corpo piezelétrico de volume $\Omega$. Para o caso em que as forças que agem sobre o sistema são superficiais, $\delta W$ é dado pela seguinte expressão:

$$
\delta W=\int_{S}\left(\delta \mathbf{u}^{T} \mathbf{F}-\delta \phi Q\right) d S
$$

onde $\mathbf{F}$ representa o vetor de forças superficiais, $Q$ é a carga elétrica por unidade de área e $\phi$ é o potencial elétrico. A entalpia $H$ é dada por:

\footnotetext{
${ }^{1}$ No entanto, é importante deixar claro que outras abordagens podem ser utilizadas, como o método de resíduos ponderados de Galerkin.
} 


$$
H=P-\mathbf{E}^{T} \mathbf{D}
$$

sendo $\mathbf{E}$ o vetor de campo elétrico, $\mathbf{D}$ o vetor de deslocamentos elétricos e $P$ a energia potencial dada por:

$$
P=\frac{1}{2} \mathbf{S}^{T} \mathbf{T}+\frac{1}{2} \mathbf{E}^{T} \mathbf{D}
$$

sendo $\mathbf{S}$ o vetor de deformações e $\mathbf{T}$ o vetor de tensões. Substituindo a Eq. (B.5) na Eq. (B.4) e substituindo a equação resultante na Eq. (B.2), obtém-se:

$$
L=\int_{\Omega}\left(\frac{1}{2} \rho \dot{\mathbf{u}}^{T} \dot{\mathbf{u}}-\frac{1}{2} \mathbf{S}^{T} \mathbf{T}+\frac{1}{2} \mathbf{E}^{T} \mathbf{D}\right) d \Omega
$$

Substituindo as equações constitutivas de um material piezelétrico, as quais se expressam segundo a Eq. (A.1) (NAILLON; COURSANT; BESNIER, 1983), na Eq. (B.6) obtém-se:

$$
L=\int_{\Omega} \frac{1}{2}\left(\rho \dot{\mathbf{u}}^{T} \dot{\mathbf{u}}-\mathbf{S}^{T} \mathbf{c}^{E} \mathbf{S}+\mathbf{S}^{T} \mathbf{e}^{T} \mathbf{E}+\mathbf{E}^{T} \mathbf{e S}+\mathbf{E}^{T} \varepsilon^{S} \mathbf{E}\right) d \Omega
$$

Substituindo as Eqs. (B.3) e (B.7) no princípio de Hamilton dado pela Eq. e integrando por partes em relação ao tempo, obtém-se a expressão variacional para problemas piezelétricos:

$$
\begin{aligned}
& \int_{\Omega}\left(-\rho \delta \mathbf{u}^{T} \ddot{\mathbf{u}}-\delta \mathbf{S}^{T} \mathbf{c}^{E} \mathbf{S}+\delta \mathbf{S}^{T} \mathbf{e}^{T} \mathbf{E}+\delta \mathbf{E}^{T} \mathbf{e} \mathbf{S}+\delta \mathbf{E}^{T} \varepsilon^{S} \mathbf{E}\right) d \Omega+ \\
& +\int_{S}\left(\delta \mathbf{u}^{T} \mathbf{F}-\delta \phi Q\right) d S=0
\end{aligned}
$$

\section{B.2 Formulação de Elementos Finitos para Problemas Piezelétricos}

$\mathrm{Na}$ análise utilizando o Método de Elementos Finitos (MEF) a região contínua de analise, $\Omega$, é dividida por um número finito de elementos menores, $V^{e}$. Esses volumes são denominados por elementos no MEF. Em cada elemento finito são definidos alguns pontos, denominados por nós, onde as incógnitas são determinadas (nos casos analisados neste estudo, os deslocamentos mecânicos e o potencial elétrico). A relação entre as incógnitas nodais de um elemento finito e as incógnitas de um ponto arbitrário, no mesmo elemento, são determinadas por funções de interpolação. Essas funções de interpolação são geralmente polinomiais (BATHE, 1996). A aproximação pelo MEF implica em assumir que 
o deslocamento $\mathbf{U}$ e o potencial elétrico $\phi$, para cada elemento finito $e$, sejam aproximados respectivamente pelos deslocamentos $\mathbf{U}_{i}$, potenciais elétricos $\boldsymbol{\Phi}_{i}$ nodais, e por funções de interpolação. Estes parâmetros podem ser expressos como:

$$
\mathbf{U}^{e}=\mathbf{N}_{u} \mathbf{U}_{i} \quad \text { e } \quad \phi^{\mathrm{e}}=\mathbf{N}_{\phi} \Phi_{\mathrm{i}}
$$

onde $\mathbf{N}_{u}$ e $\mathbf{N}_{\phi}$ são funções de interpolação ou de forma para o problema mecânico e elétrico, respectivamente. Derivando as Eq. (B.10) obtém-se a deformação $\mathbf{S}^{e}$ e o campo elétrico $\mathbf{E}^{e}$, como:

$$
\mathbf{S}^{e}=\mathbf{B}_{u} \mathbf{U}_{i} \quad \text { e } \quad \mathbf{E}^{\mathrm{e}}=-\mathbf{B}_{\phi} \boldsymbol{\Phi}_{\mathrm{i}}
$$

onde $\mathbf{B}_{u}$ e $\mathbf{B}_{\phi}$ são as matrizes deslocamento-deformação e gradiente da voltagem, respectivamente. Essas matrizes podem ser expressas como:

$$
\mathbf{B}_{u}=\left[\begin{array}{cccccc}
\frac{\partial}{\partial x} & 0 & 0 & 0 & \frac{\partial}{\partial z} & \frac{\partial}{\partial y} \\
0 & \frac{\partial}{\partial y} & 0 & \frac{\partial}{\partial z} & 0 & \frac{\partial}{\partial x} \\
& 0 & \frac{\partial}{\partial z} & \frac{\partial}{\partial y} & \frac{\partial}{\partial x} & 0
\end{array}\right]^{T} \mathbf{N}_{u} \quad \text { e } \quad \mathbf{B}_{\phi}=\left\{\begin{array}{c}
\frac{\partial}{\partial x} \\
\frac{\partial}{\partial y} \\
\frac{\partial}{\partial z}
\end{array}\right\} \mathbf{N}_{\phi}
$$

sendo $x, y$ e $z$ as coordenadas cartesianas. Substituindo as Eqs. (B.10) na Eq. (B.8), a seguinte expressão é obtida, no domínio do elemento (omitindo o subscrito $i$ ):

$$
\begin{aligned}
& \left(\delta \mathbf{u}^{e}\right)^{T}\left\{-\left(\int_{V} \rho \mathbf{N}_{u}^{T} \mathbf{N}_{u} d V^{e}\right) \ddot{\mathbf{u}}{ }^{e}-\left(\int_{V^{e}} \mathbf{B}_{u}^{T} \mathbf{c}^{E} \mathbf{B}_{u} d V^{e}\right) \mathbf{u}^{e}-\left(\int_{V} \mathbf{B}_{u}^{T} \mathbf{e}^{E} \mathbf{B}_{\phi} d V^{e}\right) \phi^{e}+\int_{S^{e}} N_{u}^{T} \mathbf{F} d S^{e}\right\}+\ldots \\
& \left(\delta \phi^{e}\right)^{T}\left\{-\left(\int_{V^{e}} \mathbf{B}_{\phi}^{T} \mathbf{e B}_{u} d V^{e}\right) \mathbf{u}^{e}-\left(\int_{V^{e}} \mathbf{B}_{\phi}^{T} \boldsymbol{\varepsilon}^{E} \mathbf{B}_{\phi} d V^{e}\right) \phi^{e}+\int_{S^{e}} N_{\phi}^{T} \mathbf{Q} d S^{e}\right\}=0
\end{aligned}
$$

onde $V^{e}$ é o volume do elemento finito $e$. Igualando a zero os termos que multiplicam $\left(\delta \mathbf{u}^{e}\right)^{T}$ e $\left(\delta \phi^{e}\right)^{T}$ na Eq. (B.12) obtém-se as equações piezelétricas descritas na forma de elementos finitos:

$$
\left[\begin{array}{cc}
\mathbf{M}^{e} & \mathbf{0} \\
\mathbf{0} & \mathbf{0}
\end{array}\right]\left\{\begin{array}{c}
\ddot{\mathbf{u}}^{e} \\
\ddot{\phi}^{e}
\end{array}\right\}+\left[\begin{array}{cc}
\mathbf{C}^{e} & \mathbf{0} \\
\mathbf{0} & \mathbf{0}
\end{array}\right]\left\{\begin{array}{c}
\dot{\mathbf{u}}^{e} \\
\dot{\phi}^{e}
\end{array}\right\}+\left[\begin{array}{cc}
\mathbf{K}_{\mathbf{u u}}^{e} & \mathbf{K}_{\mathbf{u} \phi}^{e} \\
\mathbf{K}_{\mathbf{u} \phi}^{e^{T}} & \mathbf{K}_{\phi \phi}^{e}
\end{array}\right]\left\{\begin{array}{c}
\mathbf{u}^{e} \\
\phi^{e}
\end{array}\right\}=\left\{\begin{array}{l}
\mathbf{F}^{e} \\
\mathbf{Q}^{e}
\end{array}\right\}+\left\{\begin{array}{c}
\mathbf{F}_{i}^{e} \\
\mathbf{Q}_{i}^{e}
\end{array}\right\}
$$

$\mathrm{e}$ 


$$
\begin{aligned}
& \mathbf{F}^{e}=\iint_{S^{e}} \mathbf{N}_{u}^{T} \mathbf{F} d S^{e} \quad ; \quad \mathbf{Q}^{e}=-\int_{S^{e}} \mathbf{N}_{\phi}^{T} \mathbf{Q} d S^{e} \\
& \mathbf{M}^{e}=\iiint \mathbf{N}_{u}^{T} \rho(x, y, z) \mathbf{N}_{u} d x d y d z \\
& \mathbf{K}_{u u}^{e}=\iiint \mathbf{B}_{u}^{T} \mathbf{c}^{e}(x, y, z) \mathbf{B}_{u} d x d y d z \\
& \mathbf{K}_{u \phi}^{e}=\iiint \mathbf{B}_{u}^{T} \mathbf{e}^{T}(x, y, z) \mathbf{B}_{\phi} d x d y d z \\
& \mathbf{K}_{\phi \phi}^{e}=\iiint \mathbf{B}_{\phi}^{T} \varepsilon^{S}(x, y, z) \mathbf{B}_{\phi} d x d y d z
\end{aligned}
$$

onde os termos $\mathbf{M}^{e}, \mathbf{K}_{u u}^{e}, \mathbf{K}_{u \phi}^{e}$ e $\mathbf{K}_{\phi \phi}^{e}$ são as matrizes de massa, rigidez, piezelétrica e dielétrica para um elemento finito $e$, respectivamente. Conforme a teoria de MEF, os vetores e matrizes da resposta global da estrutura resultam da montagem das contribuições de cada um dos elementos finitos (BATHE, 1996),

Assumindo-se que os deslocamentos e potenciais elétricos seguem um comportamento harmônico, isto é,

$$
\mathbf{U}=\mathbf{U}_{e} e^{i \omega t}, \quad \boldsymbol{\Phi}=\boldsymbol{\Phi}_{\mathrm{e}} \mathrm{e}^{\mathrm{i} \omega \mathrm{t}}
$$

e frequência de excitação $\omega$, o sistema piezelétrico dinâmico com amortecimento estrutural pode ser escrito na forma de elementos finitos globais da seguinte forma (BATHE, 1996):

$$
-\omega^{2}\left[\begin{array}{cc}
\mathbf{M} & \mathbf{0} \\
\mathbf{0} & \mathbf{0}
\end{array}\right]\left\{\begin{array}{l}
\mathbf{U} \\
\mathbf{\Phi}
\end{array}\right\}+i \omega\left[\begin{array}{ll}
\mathbf{C} & \mathbf{0} \\
\mathbf{0} & \mathbf{0}
\end{array}\right]\left\{\begin{array}{l}
\mathbf{U} \\
\mathbf{\Phi}
\end{array}\right\}+\left[\begin{array}{ll}
\mathbf{K}_{\mathbf{u u}} & \mathbf{K}_{\mathbf{u} \phi} \\
\mathbf{K}_{\mathbf{u} \phi}^{t} & \mathbf{K}_{\phi \phi}
\end{array}\right]\left\{\begin{array}{l}
\mathbf{U} \\
\mathbf{\Phi}
\end{array}\right\}=\left\{\begin{array}{l}
\mathbf{F} \\
\mathbf{Q}
\end{array}\right\}(
$$

onde $\omega$ é a frequência angular, $\mathbf{M}, \mathbf{C}, \mathbf{K}_{\mathbf{u u}}, \mathbf{K}_{\mathbf{u} \phi}$ e $\mathbf{K}_{\phi \phi}$ são as matrizes de massa, de amortecimento, do resistor, de rigidez, piezelétrica e dielétrica, respectivamente, e $\mathbf{U}$, $\boldsymbol{\Phi}, \mathbf{F}$ e $\mathbf{Q}$ são os vetores de deslocamento, potencial elétrico, de força e carga elétrica, respectivamente.

A matriz de amortecimento estrutural adotada é dada por:

$$
\mathbf{C}=\beta \mathbf{K}_{\mathbf{u u}}
$$

onde $\beta$ é o fator de amortecimento. É possível adicionar uma parcela proporcional a matriz de massa $\mathbf{M}$ no cálculo de $\mathbf{C}$, Porém, geralmente os dispositivos de coleta de 
energia operam nas primeiras frequências de ressonância e, portanto, a influência de $\mathbf{M}$ no cálculo do amortecimento é desprezível quando o modelo é excitado em baixas frequências. Assim, neste estudo, $\mathbf{C}$ é definido como sendo proporcional apenas a $\mathbf{K}_{\mathbf{u u}}$.

Para o caso quasi-estático não se considera a inércia e o amortecimento da estrutura e o sistema de equações é simplificado da seguinte forma:

$$
\left[\begin{array}{ll}
\mathbf{K}_{\mathbf{u u}} & \mathbf{K}_{\mathbf{u} \phi} \\
\mathbf{K}_{\mathbf{u} \phi}^{t} & \mathbf{K}_{\phi \phi}
\end{array}\right]\left\{\begin{array}{l}
\mathbf{U} \\
\boldsymbol{\Phi}
\end{array}\right\}=\left\{\begin{array}{l}
\mathbf{F} \\
\mathbf{Q}
\end{array}\right\}
$$




\section{Apêndice C - Método de Homogeneização}

Nesta seção é descrito o desenvolvimento das equações homogêneas para a piezeletricidade utilizando o método de expansão assintótica (considerando somente os termos de primeira ordem) (SANCHEZ-PALENCIA, 1980), assim como as condições de simetria utilizadas no capítulo 2.

\section{C.1 Formulação Teórica}

Considerando o procedimento padrão de homogeneização, a célula unitária é definida como $Y=\left[0, Y_{1}\right] \times\left[0, Y_{2}\right]$ e as propriedades do material $c_{i j k l}^{E}, e_{i j k}$ e $\varepsilon_{i j}^{S}$ são consideradas funções $Y$-periódicas:

$$
\begin{aligned}
& \mathbf{c}^{E \varepsilon}(\mathbf{x})=\mathbf{c}^{E}(\mathbf{x}, \mathbf{y}) ; \quad \mathbf{e}^{\varepsilon}(\mathbf{x})=\mathbf{e}(\mathbf{x}, \mathbf{y}) ; \quad \varepsilon^{S \varepsilon}(\mathbf{x})=\varepsilon^{S}(\mathbf{x}, \mathbf{y}) \\
& \mathbf{c}^{E \varepsilon}(\mathbf{x}, \mathbf{y})=\mathbf{c}^{E}(\mathbf{x}, \mathbf{y}+Y) ; \quad \mathbf{e}^{\varepsilon}(\mathbf{x}, \mathbf{y})=\mathbf{e}(\mathbf{x}, \mathbf{y}+Y) ; \quad \varepsilon^{S \varepsilon}(\mathbf{x}, \mathbf{y})=\varepsilon^{S}(\mathbf{x}, \mathbf{y}+Y)
\end{aligned}
$$

e $\mathbf{y}=\mathbf{x} / \varepsilon$ onde $\varepsilon>0$ é um parâmetro de pequeno valor que representa a micro-escala na qual as propriedades são alteradas (escala da micro-estrutura compósita), x e y são as coordenadas associadas com as dimensões macro- e micro- do material compósito, respectivamente (ver Fig. C.1).

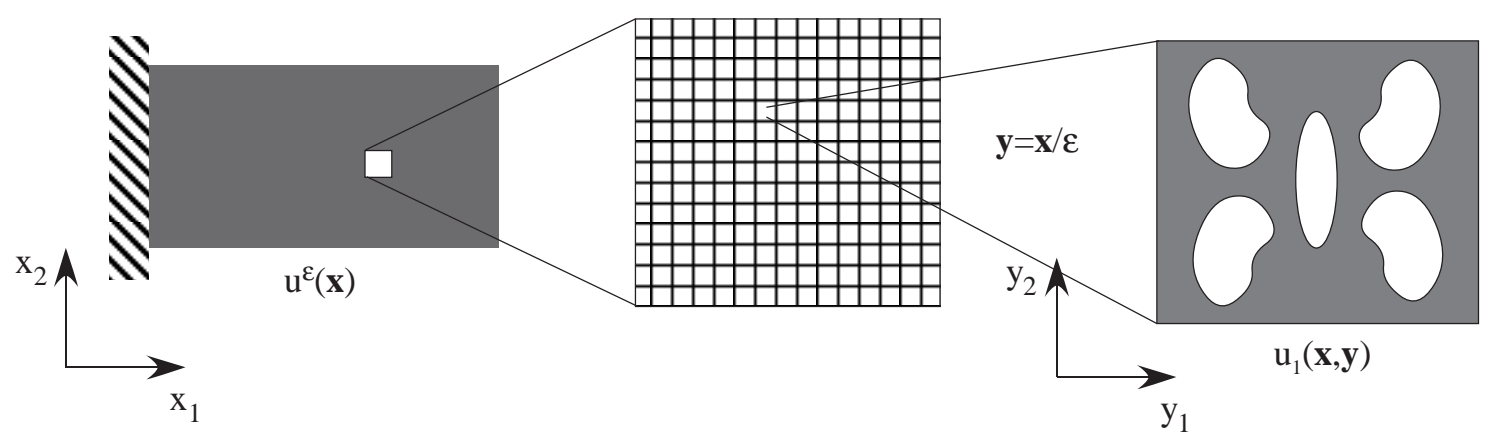

Componente (Macroescala) Microestrutura Periódica

Célula Unitária (Microescala)

Figura C.1: Conceito de Multi-escala ilustrando as dimensões macro (x) e micro (y). 
Expandindo o deslocamento do piezocompósito u e o potencial elétrico $\phi$ assintoticamente (TELEGA, 1990), obtemos que:

$$
\begin{aligned}
& \mathbf{u}^{\varepsilon}=\mathbf{u}_{0}(\mathbf{x})+\varepsilon \mathbf{u}_{1}(\mathbf{x}, \mathbf{y}) \\
& \phi^{\varepsilon}=\phi_{0}(\mathbf{x})+\varepsilon \phi_{1}(\mathbf{x}, \mathbf{y})
\end{aligned}
$$

onde somente os termos variacionais de primeira ordem são considerados, uma vez que supomos que no caso de aplicações dinâmicas, o comprimento de onda operacional é muito maior que as dimensões da célula unitária e $\mathbf{u}_{1}$ e $\phi_{1}$ são $Y$-periódicas. As tensões e os gradientes do potencial elétrico são escritas como:

$$
\begin{aligned}
\frac{\partial u_{i}^{\varepsilon}}{\partial x_{j}} & =\frac{\partial u_{0 i}(\mathbf{x})}{\partial x_{j}}+\varepsilon \frac{\partial u_{1 i}(\mathbf{x}, \mathbf{y})}{\partial x_{j}}+\frac{\partial u_{1 i}(\mathbf{x}, \mathbf{y})}{\partial y_{j}} \\
\frac{\partial \phi^{\varepsilon}}{\partial x_{j}} & =\frac{\partial \phi_{0}(\mathbf{x})}{\partial x_{j}}+\varepsilon \frac{\partial \phi_{1}(\mathbf{x}, \mathbf{y})}{\partial x_{j}}+\frac{\partial \phi_{1}(\mathbf{x}, \mathbf{y})}{\partial y_{j}} \\
\boldsymbol{\varepsilon}^{\varepsilon} & =\boldsymbol{\partial}_{\mathbf{x}} \mathbf{u}^{\varepsilon}=\boldsymbol{\partial}_{\mathbf{x}} \mathbf{u}_{0}(\mathbf{x})+\varepsilon \boldsymbol{\partial}_{\mathbf{x}} \mathbf{u}_{1}(\mathbf{x}, \mathbf{y})+\boldsymbol{\partial}_{\mathbf{y}} \mathbf{u}_{1}(\mathbf{x}, \mathbf{y}) \\
\boldsymbol{\nabla}_{\mathbf{x}} \phi^{\varepsilon} & =\boldsymbol{\nabla}_{\mathbf{x}} \phi_{0}(\mathbf{x})+\varepsilon \boldsymbol{\nabla}_{\mathbf{x}} \phi_{1}(\mathbf{x}, \mathbf{y})+\boldsymbol{\nabla}_{\mathbf{y}} \phi_{1}(\mathbf{x}, \mathbf{y})
\end{aligned}
$$

onde $\varepsilon$ é a deformação mecânica e:

$$
\left(\partial_{x}\right)_{i j}(.)=\frac{1}{2}\left(\frac{\partial(.)_{i}}{\partial x_{j}}+\frac{\partial(.)_{j}}{\partial x_{i}}\right) \quad\left(\partial_{y}\right)_{i j}(.)=\frac{1}{2}\left(\frac{\partial(.)_{i}}{\partial y_{j}}+\frac{\partial(.)_{j}}{\partial y_{i}}\right)
$$

As Eq. (C.2) e (C.3) e as propriedades (C.1) devem ser substituídas no funcional de energia para o meio piezelétrico dado pela expressão (ALLIK; HUGHES, 1970):

$$
\begin{aligned}
G\left(\mathbf{u}^{\varepsilon}, \phi^{\varepsilon}\right)= & \frac{1}{2} \int_{\Omega} \boldsymbol{\partial}_{\mathbf{x}} \mathbf{u}^{\varepsilon}: \mathbf{c}^{E \varepsilon}: \boldsymbol{\partial}_{\mathbf{x}} \mathbf{u}^{\varepsilon} d \Omega-\frac{1}{2} \int_{\Omega} \boldsymbol{\nabla}_{\mathbf{x}} \phi^{\varepsilon} \varepsilon^{s \varepsilon} \nabla_{\mathbf{x}} \phi^{\varepsilon} d \Omega+ \\
& +\int_{\Omega} \boldsymbol{\partial}_{\mathbf{x}} \mathbf{u}^{\varepsilon}: \mathbf{e}^{\varepsilon} \nabla_{\mathbf{x}} \phi^{\varepsilon} d \Omega-\int_{\Omega} \mathbf{b}^{\varepsilon} \mathbf{u}^{\varepsilon} d \Omega-\int_{\Gamma} \mathbf{t} \mathbf{u}^{\varepsilon} d \Gamma+\int_{\Gamma} d \phi^{\varepsilon} d \Gamma+ \\
& -\frac{1}{2} \int_{\Omega} \rho^{\varepsilon}\left(\dot{\mathbf{u}}^{\varepsilon}\right)^{2} d \Omega
\end{aligned}
$$

onde $\nabla_{\mathbf{x}} \phi$ é o gradiente do potencial elétrico, $\mathbf{b}$ são as forças de campo, $\mathbf{t}$ são as trações de superfície, $d$ são as cargas elétricas na superfície e $\rho$ é a densidade do meio piezelétrico. $\mathbf{c}^{E}, \boldsymbol{\varepsilon}^{s}$ e e foram definidos na Eq. (C.1) e 


$$
\mathbf{b}^{\varepsilon}=\mathbf{b}(\mathbf{x}, \mathbf{y}) \quad \rho^{\varepsilon}=\rho(\mathbf{x}, \mathbf{y})
$$

Aqui, a tração aplicada $\mathbf{t}$ e a carga elétrica de superfície $d$ são assumidas como independentes da escala da micro-estrutura $\varepsilon$. Após a substituição, temos que

$$
\begin{aligned}
G\left(\mathbf{u}^{\varepsilon}, \phi^{\varepsilon}\right)= & \frac{1}{2} \int_{\Omega}\left[\partial_{\mathbf{x}} \mathbf{u}_{0}(\mathbf{x})+\varepsilon \partial_{\mathbf{x}} \mathbf{u}_{1}(\mathbf{x}, \mathbf{y})+\partial_{\mathbf{y}} \mathbf{u}_{1}(\mathbf{x}, \mathbf{y})\right]: \mathbf{c}^{E}(\mathbf{x}, \mathbf{y}):\left[\partial_{\mathbf{x}} \mathbf{u}_{0}(\mathbf{x})+\right. \\
& \left.+\varepsilon \partial_{\mathbf{x}} \mathbf{u}_{1}(\mathbf{x}, \mathbf{y})+\partial_{\mathbf{y}} \mathbf{u}_{1}(\mathbf{x}, \mathbf{y})\right] d \Omega- \\
& -\frac{1}{2} \int_{\Omega}\left[\nabla_{\mathbf{x}} \phi_{0}(\mathbf{x})+\varepsilon \nabla_{\mathbf{x}} \phi_{1}(\mathbf{x}, \mathbf{y})+\nabla_{\mathbf{y}} \phi_{1}(\mathbf{x}, \mathbf{y})\right]: \varepsilon^{S}(\mathbf{x}, \mathbf{y})\left[\nabla_{\mathbf{x}} \phi_{0}(\mathbf{x})+\right. \\
& \left.+\varepsilon \nabla_{\mathbf{x}} \phi_{1}(\mathbf{x}, \mathbf{y})+\nabla_{\mathbf{y}} \phi_{1}(\mathbf{x}, \mathbf{y})\right] d \Omega+ \\
& +\int_{\Omega}\left[\partial_{\mathbf{x}} \mathbf{u}_{0}(\mathbf{x})+\varepsilon \partial_{\mathbf{x}} \mathbf{u}_{1}(\mathbf{x}, \mathbf{y})+\partial_{\mathbf{y}} \mathbf{u}_{1}(\mathbf{x}, \mathbf{y})\right]: \mathbf{e}(\mathbf{x}, \mathbf{y})\left[\nabla_{\mathbf{x}} \phi_{0}(\mathbf{x})+\right. \\
& \left.+\varepsilon \nabla_{\mathbf{x}} \phi_{1}(\mathbf{x}, \mathbf{y})+\nabla_{\mathbf{y}} \phi_{1}(\mathbf{x}, \mathbf{y})\right] d \Omega- \\
& -\int_{\Omega} \mathbf{b}(\mathbf{x}, \mathbf{y})\left(\mathbf{u}_{0}(\mathbf{x})+\varepsilon \mathbf{u}_{1}(\mathbf{x}, \mathbf{y})\right) d \Omega-\int_{\Gamma} \mathbf{t}\left(\mathbf{u}_{0}(\mathbf{x})+\varepsilon \mathbf{u}_{1}(\mathbf{x}, \mathbf{y})\right) d \Gamma+ \\
& +\int_{\Gamma} d\left(\phi_{0}(\mathbf{x})+\varepsilon \phi_{1}(\mathbf{x}, \mathbf{y})\right) d \Gamma-\frac{1}{2} \int_{\Omega} \rho(\mathbf{x}, \mathbf{y})\left(\dot{\mathbf{u}}_{0}(\mathbf{x})+\varepsilon \dot{\mathbf{u}}_{1}(\mathbf{x}, \mathbf{y})\right)^{2} d \Omega
\end{aligned}
$$

$$
\begin{aligned}
G\left(\mathbf{u}^{\varepsilon}, \phi^{\varepsilon}\right)= & \frac{1}{2} \int_{\Omega}\left[\partial_{\mathbf{x}} \mathbf{u}_{0}(\mathbf{x})+\partial_{\mathbf{y}} \mathbf{u}_{1}(\mathbf{x}, \mathbf{y})\right]: \mathbf{c}^{E}(\mathbf{x}, \mathbf{y}):\left[\partial_{\mathbf{x}} \mathbf{u}_{0}(\mathbf{x})+\partial_{\mathbf{y}} \mathbf{u}_{1}(\mathbf{x}, \mathbf{y})\right] d \Omega+ \\
& +\varepsilon \int_{\Omega} \partial_{\mathbf{x}} \mathbf{u}_{1}(\mathbf{x}, \mathbf{y}): \mathbf{c}^{E}(\mathbf{x}, \mathbf{y}):\left[\partial_{\mathbf{x}} \mathbf{u}_{0}(\mathbf{x})+\partial_{\mathbf{y}} \mathbf{u}_{1}(\mathbf{x}, \mathbf{y})\right] d \Omega+ \\
& +\frac{\varepsilon^{2}}{2} \int_{\Omega} \partial_{\mathbf{x}} \mathbf{u}_{1}(\mathbf{x}, \mathbf{y}): \mathbf{c}^{E}(\mathbf{x}, \mathbf{y}): \partial_{\mathbf{x}} \mathbf{u}_{1}(\mathbf{x}, \mathbf{y}) d \Omega- \\
& -\frac{1}{2} \int_{\Omega}\left[\nabla_{\mathbf{x}} \phi_{0}(\mathbf{x})+\nabla_{\mathbf{y}} \phi_{1}(\mathbf{x}, \mathbf{y})\right] \varepsilon^{S}(\mathbf{x}, \mathbf{y})\left[\nabla_{\mathbf{x}} \phi_{0}(\mathbf{x})+\nabla_{\mathbf{y}} \phi_{1}(\mathbf{x}, \mathbf{y})\right] d \Omega- \\
& -\varepsilon \int_{\Omega} \nabla_{\mathbf{x}} \phi_{1}(\mathbf{x}, \mathbf{y}) \varepsilon^{S}(\mathbf{x}, \mathbf{y})\left[\nabla_{\mathbf{x}} \phi_{0}(\mathbf{x})+\nabla_{\mathbf{y}} \phi_{1}(\mathbf{x}, \mathbf{y})\right] d \Omega- \\
& -\frac{\varepsilon^{2}}{2} \int_{\Omega} \nabla_{\mathbf{x}} \phi_{1}(\mathbf{x}, \mathbf{y}) \varepsilon^{S}(\mathbf{x}, \mathbf{y}) \nabla_{\mathbf{x}} \phi_{1}(\mathbf{x}, \mathbf{y}) d \Omega+ \\
& +\int_{\Omega}\left[\partial_{\mathbf{x}} \mathbf{u}_{0}(\mathbf{x})+\partial_{\mathbf{y}} \mathbf{u}_{1}(\mathbf{x}, \mathbf{y})\right]: \mathbf{e}(\mathbf{x}, \mathbf{y})\left[\nabla_{\mathbf{x}} \phi_{0}(\mathbf{x})+\nabla_{\mathbf{y}} \phi_{1}(\mathbf{x}, \mathbf{y})\right] d \Omega+ \\
& +\varepsilon \int_{\Omega} \partial_{\mathbf{x}} \mathbf{u}_{1}(\mathbf{x}, \mathbf{y}): \mathbf{e}(\mathbf{x}, \mathbf{y})\left[\nabla_{\mathbf{x}} \phi_{0}(\mathbf{x})+\nabla_{\mathbf{y}} \phi_{1}(\mathbf{x}, \mathbf{y})\right] d \Omega+ \\
& +\varepsilon \int_{\Omega}\left[\partial_{\mathbf{x}} \mathbf{u}_{0}(\mathbf{x})+\partial_{\mathbf{y}} \mathbf{u}_{1}(\mathbf{x}, \mathbf{y})\right]: \mathbf{e}(\mathbf{x}, \mathbf{y}) \nabla_{\mathbf{x}} \phi_{1}(\mathbf{x}, \mathbf{y}) d \Omega+ \\
+ & \varepsilon^{2} \int_{\Omega} \partial_{\mathbf{x}} \mathbf{u}_{1}(\mathbf{x}, \mathbf{y}): \mathbf{e}(\mathbf{x}, \mathbf{y}) \nabla_{\mathbf{x}} \phi_{1}(\mathbf{x}, \mathbf{y}) d \Omega-
\end{aligned}
$$




$$
\begin{aligned}
& -\int_{\Omega} \mathbf{b}(\mathbf{x}, \mathbf{y}) \mathbf{u}_{0}(\mathbf{x}) d \Omega-\varepsilon \int_{\Omega} \mathbf{b}(\mathbf{x}, \mathbf{y}) \mathbf{u}_{1}(\mathbf{x}, \mathbf{y}) d \Omega- \\
& -\int_{\Gamma} \mathbf{t} \mathbf{u}_{0}(\mathbf{x}) d \Gamma-\varepsilon \int_{\Gamma} \mathbf{t u}_{1}(\mathbf{x}, \mathbf{y}) d \Gamma+ \\
& +\int_{\Gamma} d \phi_{0}(\mathbf{x}) d \Gamma+\varepsilon \int_{\Gamma} d \phi_{1}(\mathbf{x}, \mathbf{y}) d \Gamma-\frac{1}{2} \int_{\Omega} \rho(\mathbf{x}, \mathbf{y})\left(\dot{\mathbf{u}}_{0}(\mathbf{x})\right)^{2} d \Omega+ \\
& -\varepsilon \int_{\Omega} \rho(\mathbf{x}, \mathbf{y}) \dot{\mathbf{u}}_{0}(\mathbf{x}) \dot{\mathbf{u}}_{1}(\mathbf{x}, \mathbf{y}) d \Omega-\frac{\varepsilon^{2}}{2} \int_{\Omega} \rho(\mathbf{x}, \mathbf{y})\left(\dot{\mathbf{u}}_{1}(\mathbf{x}, \mathbf{y})\right)^{2} d \Omega
\end{aligned}
$$

Calculando o diferencial-G de $G\left(\mathbf{u}^{\varepsilon}, \phi^{\varepsilon}\right)$ para $\mathbf{u}^{\varepsilon}$ e $\phi^{\varepsilon}$ e calculando o limite de $\varepsilon \rightarrow 0$ em $G\left(\mathbf{u}^{\varepsilon}, \phi^{\varepsilon}\right)$ :

$$
\begin{aligned}
& \lim _{\varepsilon \rightarrow 0}\left\{\delta G\left(\mathbf{u}^{\varepsilon}, \phi^{\varepsilon}\right)\right\}= \\
& =\lim _{\varepsilon \rightarrow 0}\left\{\int_{\Omega}\left[\partial_{\mathbf{x}} \delta \mathbf{u}_{0}(\mathbf{x})+\partial_{\mathbf{y}} \delta \mathbf{u}_{1}(\mathbf{x}, \mathbf{y})\right]: \mathbf{c}^{E}(\mathbf{x}, \mathbf{y}):\left[\partial_{\mathbf{x}} \mathbf{u}_{0}(\mathbf{x})+\partial_{\mathbf{y}} \mathbf{u}_{1}(\mathbf{x}, \mathbf{y})\right] d \Omega-\right. \\
& -\int_{\Omega}\left[\nabla_{\mathbf{x}} \delta \phi_{0}(\mathbf{x})+\nabla_{\mathbf{y}} \delta \phi_{1}(\mathbf{x}, \mathbf{y})\right] \varepsilon^{S}(\mathbf{x}, \mathbf{y})\left[\nabla_{\mathbf{x}} \phi_{0}(\mathbf{x})+\nabla_{\mathbf{y}} \phi_{1}(\mathbf{x}, \mathbf{y})\right] d \Omega+ \\
& +\int_{\Omega}\left[\partial_{\mathbf{x}} \delta \mathbf{u}_{0}(\mathbf{x})+\partial_{\mathbf{y}} \delta \mathbf{u}_{1}(\mathbf{x}, \mathbf{y})\right]: \mathbf{e}(\mathbf{x}, \mathbf{y})\left[\nabla_{\mathbf{x}} \phi_{0}(\mathbf{x})+\nabla_{\mathbf{y}} \phi_{1}(\mathbf{x}, \mathbf{y})\right] d \Omega+ \\
& +\int_{\Omega}\left[\partial_{\mathbf{x}} \mathbf{u}_{0}(\mathbf{x})+\partial_{\mathbf{y}} \mathbf{u}_{1}(\mathbf{x}, \mathbf{y})\right]: \mathbf{e}(\mathbf{x}, \mathbf{y})\left[\nabla_{\mathbf{x}} \delta \phi_{0}(\mathbf{x})+\nabla_{\mathbf{y}} \delta \phi_{1}(\mathbf{x}, \mathbf{y})\right] d \Omega- \\
& -\int_{\Omega} \mathbf{b}(\mathbf{x}, \mathbf{y}) \delta \mathbf{u}_{0}(\mathbf{x}) d \Omega-\int_{\Gamma} \mathbf{t} \delta \mathbf{u}_{0}(\mathbf{x}) d \Gamma+\int_{\Gamma} d \delta \phi_{0}(\mathbf{x}) d \Gamma- \\
& \left.-\int_{\Omega} \rho(\mathbf{x}, \mathbf{y}) \dot{\mathbf{u}}_{0}(\mathbf{x}) \delta \dot{\mathbf{u}}_{0}(\mathbf{x}) d \Omega\right\}=0
\end{aligned}
$$

para todo os valores admissíveis de $\delta \mathbf{u}_{0}(\mathbf{x}), \delta \mathbf{u}_{1}(\mathbf{x}, \mathbf{y}), \delta \phi_{0}(\mathbf{x})$ e $\delta \phi_{1}(\mathbf{x}, \mathbf{y})$. Considerando que (SANCHEZ-PALENCIA, 1980)

$$
\lim _{\varepsilon \rightarrow 0} \int_{\Omega} \Phi\left(\mathbf{x}, \frac{\mathbf{x}}{\varepsilon}\right) d \Omega=\frac{1}{|Y|} \int_{\Omega} \int_{Y} \Phi(\mathbf{x}, \mathbf{y}) d Y d \Omega
$$

onde $\Phi$ denota o funcional de interesse e

$$
\begin{aligned}
\int_{t_{1}}^{t_{2}} \dot{\mathbf{u}}_{0}(\mathbf{x}, t) \delta \dot{\mathbf{u}}_{0}(\mathbf{x}, t) d t & =\left.\dot{\mathbf{u}}_{0}(\mathbf{x}, t) \delta \mathbf{u}_{0}(\mathbf{x}, t)\right|_{t_{1}} ^{t_{2}}-\int_{t_{1}}^{t_{2}} \ddot{\mathbf{u}}_{0}(\mathbf{x}, t) \delta \mathbf{u}_{0}(\mathbf{x}, t) d t= \\
& =-\int_{t_{1}}^{t_{2}} \ddot{\mathbf{u}}_{0}(\mathbf{x}, t) \delta \mathbf{u}_{0}(\mathbf{x}, t) d t
\end{aligned}
$$


$\left(\right.$ pois $\delta \mathbf{u}_{0}\left(\mathbf{x}, t_{1}\right)=\delta \mathbf{u}_{0}\left(\mathbf{x}, t_{2}\right)=0$ ), e uma vez que $\delta \mathbf{u}_{0}(\mathbf{x}), \delta \mathbf{u}_{1}(\mathbf{x}, \mathbf{y}), \delta \phi_{0}(\mathbf{x})$ e $\delta \phi_{1}(\mathbf{x}, \mathbf{y})$ são independentes, as seguintes equações devem ser satisfeitas (SANCHEZ-PALENCIA, 1980):

$$
\begin{gathered}
\int_{\Omega} \frac{1}{|Y|} \int_{Y} \partial_{\mathbf{x}} \delta \mathbf{u}_{0}(\mathbf{x}): \mathbf{c}^{E}(\mathbf{x}, \mathbf{y}):\left[\partial_{\mathbf{x}} \mathbf{u}_{0}(\mathbf{x})+\partial_{\mathbf{y}} \mathbf{u}_{1}(\mathbf{x}, \mathbf{y})\right] d Y d \Omega+ \\
+\int_{\Omega} \frac{1}{|Y|} \int_{Y} \partial_{\mathbf{x}} \delta \mathbf{u}_{0}(\mathbf{x}): \mathbf{e}(\mathbf{x}, \mathbf{y})\left[\nabla_{\mathbf{x}} \phi_{0}(\mathbf{x})+\nabla_{\mathbf{y}} \phi_{1}(\mathbf{x}, \mathbf{y})\right] d Y d \Omega- \\
-\int_{\Omega} \frac{1}{|Y|} \int_{Y} \mathbf{b}(\mathbf{x}, \mathbf{y}) \delta \mathbf{u}_{0}(\mathbf{x}) d Y d \Omega-\int_{\Gamma} \mathbf{t} \delta \mathbf{u}_{0}(\mathbf{x}) d \Gamma+ \\
+\int_{\Omega} \frac{1}{|Y|} \int_{Y} \rho(\mathbf{x}, \mathbf{y}) \ddot{\mathbf{u}}_{0}(\mathbf{x}) \delta \mathbf{u}_{0}(\mathbf{x}) d Y d \Omega=0 \\
\int_{\Omega} \frac{1}{|Y|} \int_{Y}\left[\partial_{\mathbf{x}} \mathbf{u}_{0}(\mathbf{x})+\partial_{\mathbf{y}} \mathbf{u}_{1}(\mathbf{x}, \mathbf{y})\right]: \mathbf{e}(\mathbf{x}, \mathbf{y}) \nabla_{\mathbf{x}} \delta \phi_{0}(\mathbf{x}) d Y d \Omega- \\
-\int_{\Omega} \frac{1}{|Y|} \int_{Y}\left[\nabla_{\mathbf{x}} \phi_{0}(\mathbf{x})+\nabla_{\mathbf{y}} \phi_{1}(\mathbf{x}, \mathbf{y})\right] \varepsilon^{S}(\mathbf{x}, \mathbf{y}) \nabla_{\mathbf{x}} \delta \phi_{0}(\mathbf{x}) d Y d \Omega+ \\
+\int_{\Gamma} d \delta \phi(\mathbf{x}) d \Gamma=0
\end{gathered}
$$

para qualquer valor admissível de $\delta \mathbf{u}_{0}(\mathbf{x})$ e $\delta \phi_{0}(\mathbf{x})$, e:

$$
\begin{aligned}
& \int_{\Omega} \frac{1}{|Y|} \int_{Y} \partial_{\mathbf{y}} \delta \mathbf{u}_{1}(\mathbf{x}, \mathbf{y}): \mathbf{c}^{E}(\mathbf{x}, \mathbf{y}):\left[\partial_{\mathbf{x}} \mathbf{u}_{0}(\mathbf{x})+\partial_{\mathbf{y}} \mathbf{u}_{1}(\mathbf{x}, \mathbf{y})\right] d Y d \Omega+ \\
& +\int_{\Omega} \frac{1}{|Y|} \int_{Y} \partial_{\mathbf{y}} \delta \mathbf{u}_{1}(\mathbf{x}, \mathbf{y}): \mathbf{e}(\mathbf{x}, \mathbf{y})\left[\nabla_{\mathbf{x}} \phi_{0}(\mathbf{x})+\nabla_{\mathbf{y}} \phi_{1}(\mathbf{x}, \mathbf{y})\right] d Y d \Omega=0 \\
& \int_{\Omega} \frac{1}{|Y|} \int_{Y}\left[\partial_{\mathbf{x}} \mathbf{u}_{0}(\mathbf{x})+\partial_{\mathbf{y}} \mathbf{u}_{1}(\mathbf{x}, \mathbf{y})\right]: \mathbf{e}(\mathbf{x}, \mathbf{y}) \nabla_{\mathbf{y}} \delta \phi_{1}(\mathbf{x}, \mathbf{y}) d Y d \Omega- \\
& -\int_{\Omega} \frac{1}{|Y|} \int_{Y}\left[\nabla_{\mathbf{x}} \phi_{0}(\mathbf{x})+\nabla_{\mathbf{y}} \phi_{1}(\mathbf{x}, \mathbf{y})\right] \boldsymbol{\varepsilon}^{S}(\mathbf{x}, \mathbf{y}) \nabla_{\mathbf{y}} \delta \phi_{1}(\mathbf{x}, \mathbf{y}) d Y d \Omega=0
\end{aligned}
$$

para qualquer valor admissível de $\delta \mathbf{u}_{1}(\mathbf{x}, \mathbf{y})$ e $\delta \phi_{1}(\mathbf{x}, \mathbf{y})$.

As Eq. (C.12) e (C.13) são denominadas por equações macroscópicas, uma vez que contém termos relacionados a $\delta \mathbf{u}_{0}(\mathbf{x})$ e $\delta \phi_{0}(\mathbf{x})$, enquanto que as Eq. (C.14) e (C.15) são denominadas por equações microscópicas, uma vez que contém termos relacionados a $\delta \mathbf{u}_{1}(\mathbf{x}, \mathbf{y})$ e $\delta \phi_{1}(\mathbf{x}, \mathbf{y})$. Devido à linearidade do problema e assumindo a separação das variáveis por $\mathbf{u}_{1}(\mathbf{x}, \mathbf{y})$ e $\phi_{1}(\mathbf{x}, \mathbf{y})$ (TELEGA, 1990), obtém-se que:

$$
\mathbf{u}_{1}=\chi(\mathbf{x}, \mathbf{y}) \varepsilon\left(\mathbf{u}_{0}(\mathbf{x})\right)+\mathbf{\Phi}(\mathbf{x}, \mathbf{y}) \nabla_{\mathbf{x}} \phi_{0}(\mathbf{x})
$$




$$
\begin{aligned}
\phi_{1} & =\psi(\mathbf{x}, \mathbf{y}) \varepsilon\left(\mathbf{u}_{0}(\mathbf{x})\right)+R(\mathbf{x}, \mathbf{y}) \boldsymbol{\nabla}_{\mathbf{x}} \phi_{0}(\mathbf{x}) \\
\boldsymbol{\partial}_{\mathbf{y}} \mathbf{u}_{1}(\mathbf{x}, \mathbf{y}) & =\boldsymbol{\partial}_{\mathbf{y}} \boldsymbol{\chi}(\mathbf{x}, \mathbf{y}) \boldsymbol{\partial}_{\mathbf{x}} \mathbf{u}_{0}(\mathbf{x})+\boldsymbol{\partial}_{\mathbf{y}} \boldsymbol{\Phi}(\mathbf{x}, \mathbf{y}) \boldsymbol{\nabla}_{\mathbf{x}} \phi_{0}(\mathbf{x}) \\
\boldsymbol{\nabla}_{\mathbf{y}} \phi_{1}(\mathbf{x}, \mathbf{y}) & =\boldsymbol{\nabla}_{\mathbf{y}} \psi(\mathbf{x}, \mathbf{y}) \boldsymbol{\partial}_{\mathbf{x}} \mathbf{u}_{0}(\mathbf{x})+\boldsymbol{\nabla}_{\mathbf{y}} R(\mathbf{x}, \mathbf{y}) \boldsymbol{\nabla}_{\mathbf{x}} \phi_{0}(\mathbf{x})
\end{aligned}
$$

onde $\chi(\mathbf{x}, \mathbf{y})$ é o deslocamento característico da célula unitária, $R(\mathbf{x}, \mathbf{y})$ é o potencial elétrico da célula unitária e $\psi(\mathbf{x}, \mathbf{y})$ e $\boldsymbol{\Phi}(\mathbf{x}, \mathbf{y})$ são as funções "acopladas" características da célula unitária. Todas estas funções são $Y$-periódicas; $\boldsymbol{\chi}(\mathbf{x}, \mathbf{y})$ e $\boldsymbol{\Phi}(\mathbf{x}, \mathbf{y})$ pertencem a $H_{p e r}\left(Y, R^{3}\right)$, e $\psi(\mathbf{x}, \mathbf{y})$ e $R(\mathbf{x}, \mathbf{y})$ pertencem a $H_{p e r}(Y)$, onde:

$$
\begin{aligned}
H_{p e r}(Y) & =\left\{v \in H^{1}(Y) \mid v \text { obtém valores iguais em lados opostos de } Y\right\}, \\
H_{\text {per }}\left(Y, R^{3}\right) & =\left\{\mathbf{v}=\left(v_{i}\right) \mid v_{i} \in H_{\text {per }}(Y), i=1,2,3\right\}
\end{aligned}
$$

que corresponde à condição de periodicidade da célula unitária (ver Fig. C.2).

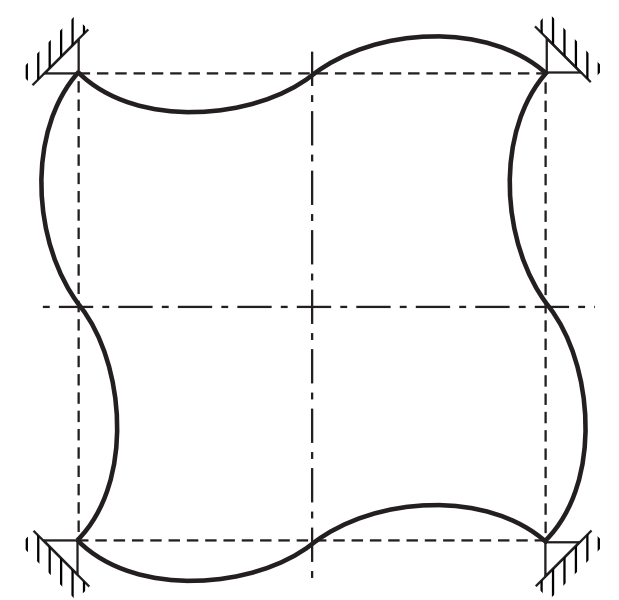

Figura C.2: Condições periódicas na célula unitária.

Portanto, substituindo a Eq. (C.17) nas equações microscópicas (C.14) e (C.15) tem-se que:

$$
\begin{gathered}
\int_{\Omega} \frac{1}{|Y|} \int_{Y} \partial_{\mathbf{y}} \delta \mathbf{u}_{1}(\mathbf{x}, \mathbf{y}): \mathbf{c}^{E}(\mathbf{x}, \mathbf{y}):\left[\partial_{\mathbf{x}} \mathbf{u}_{0}(\mathbf{x})+\partial_{\mathbf{y}} \chi(\mathbf{x}, \mathbf{y}) \partial_{\mathbf{x}} \mathbf{u}_{0}(\mathbf{x})+\right. \\
\left.+\partial_{\mathbf{y}} \mathbf{\Phi}(\mathbf{x}, \mathbf{y}) \nabla_{\mathbf{x}} \phi_{0}(\mathbf{x})\right] d Y d \Omega+ \\
+\int_{\Omega} \frac{1}{|Y|} \int_{Y} \partial_{\mathbf{y}} \delta \mathbf{u}_{1}(\mathbf{x}, \mathbf{y}): \mathbf{e}(\mathbf{x}, \mathbf{y})\left[\nabla_{\mathbf{x}} \phi_{0}(\mathbf{x})+\nabla_{\mathbf{y}} \boldsymbol{\psi}(\mathbf{x}, \mathbf{y}) \partial_{\mathbf{x}} \mathbf{u}_{0}(\mathbf{x})+\right. \\
\left.+\nabla_{\mathbf{y}} R(\mathbf{x}, \mathbf{y}) \nabla_{\mathbf{x}} \phi_{0}(\mathbf{x})\right] d Y d \Omega=0 \\
\forall \delta \mathbf{u}_{1} \in H_{p e r}\left(Y, R^{3}\right) \\
\int_{\Omega} \frac{1}{|Y|} \int_{Y}\left[\partial_{\mathbf{x}} \mathbf{u}_{0}(\mathbf{x})+\partial_{\mathbf{y}} \chi(\mathbf{x}, \mathbf{y}) \partial_{\mathbf{x}} \mathbf{u}_{0}(\mathbf{x})+\right.
\end{gathered}
$$




$$
\begin{aligned}
& \left.+\partial_{\mathbf{y}} \mathbf{\Phi}(\mathbf{x}, \mathbf{y}) \nabla_{\mathbf{x}} \phi_{0}(\mathbf{x})\right]: \mathbf{e}(\mathbf{x}, \mathbf{y}) \nabla_{\mathbf{y}} \delta \phi_{1}(\mathbf{x}, \mathbf{y}) d Y d \Omega- \\
& \quad-\int_{\Omega} \frac{1}{|Y|} \int_{Y}\left[\nabla_{\mathbf{x}} \phi_{0}(\mathbf{x})+\nabla_{\mathbf{y}} \boldsymbol{\psi}(\mathbf{x}, \mathbf{y}) \partial_{\mathbf{x}} \mathbf{u}_{0}(\mathbf{x})+\right. \\
& \left.+\nabla_{\mathbf{y}} R(\mathbf{x}, \mathbf{y}) \nabla_{\mathbf{x}} \phi_{0}(\mathbf{x})\right] \boldsymbol{\varepsilon}^{S}(\mathbf{x}, \mathbf{y}) \nabla_{\mathbf{y}} \delta \phi_{1}(\mathbf{x}, \mathbf{y}) d Y d \Omega=0
\end{aligned}
$$

$\forall \delta \phi_{1} \in H_{\text {per }}(Y)$

As equações microscópicas (C.18) e (C.19) podem ser satisfeitas (GALKA; TELEGA; WOJNAR, 1992) se $\boldsymbol{\chi}(\mathbf{x}, \mathbf{y}), \mathbf{\Phi}(\mathbf{x}, \mathbf{y}), \psi(\mathbf{x}, \mathbf{y})$ e $R(\mathbf{x}, \mathbf{y})$ forem soluções de:

$$
\begin{array}{r}
\frac{1}{|Y|} \int_{Y}\left[\left(\mathbf{I}+\boldsymbol{\partial}_{\mathbf{y}} \boldsymbol{\chi}(\mathbf{x}, \mathbf{y})\right): \mathbf{c}^{E}(\mathbf{x}, \mathbf{y})+\boldsymbol{\nabla}_{\mathbf{y}} \psi(\mathbf{x}, \mathbf{y}) \mathbf{e}(\mathbf{x}, \mathbf{y})\right]: \boldsymbol{\partial}_{\mathbf{y}} \delta \mathbf{u}_{1}(\mathbf{x}, \mathbf{y}) d Y=0 \\
\forall \delta \mathbf{u}_{1} \in H_{p e r}\left(Y, R^{3}\right) \\
\frac{1}{|Y|} \int_{Y}\left[\left(\mathbf{I}+\boldsymbol{\partial}_{\mathbf{y}} \boldsymbol{\chi}(\mathbf{x}, \mathbf{y})\right): \mathbf{e}(\mathbf{x}, \mathbf{y})-\boldsymbol{\nabla}_{\mathbf{y}} \psi(\mathbf{x}, \mathbf{y}) \boldsymbol{\varepsilon}^{s}(\mathbf{x}, \mathbf{y})\right] \boldsymbol{\nabla}_{\mathbf{y}} \delta \phi_{1}(\mathbf{x}, \mathbf{y}) d Y=0 \\
\forall \delta \phi_{1} \in H_{p e r}(Y) \\
\frac{1}{|Y|} \int_{Y}\left[\boldsymbol{\partial}_{\mathbf{y}} \mathbf{\Phi}(\mathbf{x}, \mathbf{y}): \mathbf{c}^{E}(\mathbf{x}, \mathbf{y})+\left(\mathbf{I}+\boldsymbol{\nabla}_{\mathbf{y}} R(\mathbf{x}, \mathbf{y})\right) \mathbf{e}(\mathbf{x}, \mathbf{y})\right]: \boldsymbol{\partial}_{\mathbf{y}} \delta \mathbf{u}_{1}(\mathbf{x}, \mathbf{y}) d Y=0 \\
\forall \delta \mathbf{u}_{1} \in H_{p e r}\left(Y, R^{3}\right) \\
\frac{1}{|Y|} \int_{Y}\left[\boldsymbol{\partial}_{\mathbf{y}} \mathbf{\Phi}(\mathbf{x}, \mathbf{y}): \mathbf{e}(\mathbf{x}, \mathbf{y})-\left(\mathbf{I}+\boldsymbol{\nabla}_{\mathbf{y}} R(\mathbf{x}, \mathbf{y})\right) \boldsymbol{\varepsilon}^{s}(\mathbf{x}, \mathbf{y})\right] \boldsymbol{\nabla}_{\mathbf{y}} \delta \phi_{1}(\mathbf{x}, \mathbf{y}) d Y=0 \\
\forall \delta \phi_{1} \in H_{p e r}(Y)
\end{array}
$$

ou, utilizando a notação indexada:

$$
\begin{array}{r}
\frac{1}{|Y|} \int_{Y}\left[c_{i j k l}^{E}(\mathbf{x}, \mathbf{y})\left(\delta_{i m} \delta_{j n}+\frac{\partial \chi_{i}^{(m n)}}{\partial y_{j}}\right)+e_{i k l}(\mathbf{x}, \mathbf{y}) \frac{\partial \psi^{(m n)}}{\partial y_{i}}\right] \varepsilon_{k l}(\mathbf{v}) d Y=0 \\
\forall \mathbf{v} \in H_{p e r}\left(Y, R^{3}\right) \\
\frac{1}{|Y|} \int_{Y}\left[e_{k i j}(\mathbf{x}, \mathbf{y})\left(\delta_{i m} \delta_{j n}+\frac{\partial \chi_{i}^{(m n)}}{\partial y_{j}}\right)-\varepsilon_{i k}^{S}(\mathbf{x}, \mathbf{y}) \frac{\partial \psi^{(m n)}}{\partial y_{i}}\right] \frac{\partial \varphi}{\partial y_{k}} d Y=0 \\
\forall \varphi \in H_{p e r}(Y) \\
\frac{1}{|Y|} \int_{Y}\left[c_{k l i j}^{E}(\mathbf{x}, \mathbf{y}) \frac{\partial \Phi_{k}^{(m)}}{\partial y_{l}}+e_{k i j}(\mathbf{x}, \mathbf{y})\left(\delta_{m k}+\frac{\partial R^{(m)}}{\partial y_{k}}\right)\right] \varepsilon_{i j}(\mathbf{v}) d Y=0 \\
\forall \mathbf{v} \in H_{p e r}\left(Y, R^{3}\right) \\
\frac{1}{|Y|} \int_{Y}\left[e_{k i j}(\mathbf{x}, \mathbf{y}) \frac{\partial \Phi_{i}^{(m)}}{\partial y_{j}}-\varepsilon_{i k}^{S}(\mathbf{x}, \mathbf{y})\left(\delta_{m i}+\frac{\partial R^{(m)}}{\partial y_{i}}\right)\right] \frac{\partial \varphi}{\partial y_{k}} d Y=0 \\
\forall \varphi \in H_{p e r}(Y)
\end{array}
$$


Substituindo a Eq. (C.17) nas equações macroscópicas (C.12) e (C.13) obtém-se que:

$$
\begin{gathered}
\int_{\Omega} \frac{1}{|Y|} \int_{Y} \partial_{\mathbf{x}} \delta \mathbf{u}_{0}(\mathbf{x}): \mathbf{c}^{E}(\mathbf{x}, \mathbf{y}):\left[\partial_{\mathbf{x}} \mathbf{u}_{0}(\mathbf{x})+\partial_{\mathbf{y}} \chi(\mathbf{x}, \mathbf{y}) \partial_{\mathbf{x}} \mathbf{u}_{0}(\mathbf{x})+\right. \\
\left.+\partial_{\mathbf{y}} \mathbf{\Phi}(\mathbf{x}, \mathbf{y}) \nabla_{\mathbf{x}} \phi_{0}(\mathbf{x})\right] d Y d \Omega+ \\
+\int_{\Omega} \frac{1}{|Y|} \int_{Y} \partial_{\mathbf{x}} \delta \mathbf{u}_{0}(\mathbf{x}): \mathbf{e}(\mathbf{x}, \mathbf{y})\left[\nabla_{\mathbf{x}} \phi_{0}(\mathbf{x})+\nabla_{\mathbf{y}} \psi(\mathbf{x}, \mathbf{y}) \partial_{\mathbf{x}} \mathbf{u}_{0}(\mathbf{x})+\right. \\
\left.+\nabla_{\mathbf{y}} R(\mathbf{x}, \mathbf{y}) \nabla_{\mathbf{x}} \phi_{0}(\mathbf{x})\right] d Y d \Omega- \\
-\int_{\Omega} \frac{1}{|Y|} \int_{Y} \mathbf{b}(\mathbf{x}, \mathbf{y}) \delta \mathbf{u}_{0}(\mathbf{x}) d Y d \Omega-\int_{\Gamma} \mathbf{t} \delta \mathbf{u}_{0}(\mathbf{x}) d \Gamma+ \\
+\int_{\Omega} \frac{1}{|Y|} \int_{Y} \rho(\mathbf{x}, \mathbf{y}) \ddot{\mathbf{u}}_{0}(\mathbf{x}) \delta \mathbf{u}_{0}(\mathbf{x}) d Y d \Omega=0 \\
\int_{\Omega} \frac{1}{|Y|} \int_{Y}\left[\partial_{\mathbf{x}} \mathbf{u}_{0}(\mathbf{x})+\partial_{\mathbf{y}} \chi(\mathbf{x}, \mathbf{y}) \partial_{\mathbf{x}} \mathbf{u}_{0}(\mathbf{x})+\right. \\
\left.\partial_{\mathbf{y}} \mathbf{\Phi}(\mathbf{x}, \mathbf{y}) \nabla_{\mathbf{x}} \phi_{0}(\mathbf{x})\right]: \mathbf{e}(\mathbf{x}, \mathbf{y}) \nabla_{\mathbf{x}} \delta \phi_{0}(\mathbf{x}) d Y d \Omega- \\
-\int_{\Omega} \frac{1}{|Y|} \int_{Y}\left[\nabla_{\mathbf{x}} \phi_{0}(\mathbf{x})+\nabla_{\mathbf{y}} \psi(\mathbf{x}, \mathbf{y}) \partial_{\mathbf{x}} \mathbf{u}_{0}(\mathbf{x})+\right. \\
\left.\nabla_{\mathbf{y}} R(\mathbf{x}, \mathbf{y}) \nabla_{\mathbf{x}} \phi_{0}(\mathbf{x})\right] \varepsilon^{S}(\mathbf{x}, \mathbf{y}) \nabla_{\mathbf{x}} \delta \phi_{0}(\mathbf{x}) d Y d \Omega+\int_{\Gamma} d \delta \phi_{0}(\mathbf{x}) d \Gamma=0
\end{gathered}
$$

Portanto, considerando as propriedades efetivas dadas por:

$$
\begin{aligned}
\mathbf{c}_{H}^{E} & =\frac{1}{|Y|}\left\{\int_{Y}\left[\mathbf{c}^{E}(\mathbf{x}, \mathbf{y}):\left(\mathbf{I}+\boldsymbol{\partial}_{\mathbf{y}} \boldsymbol{\chi}(\mathbf{x}, \mathbf{y})\right)+\mathbf{e}(\mathbf{x}, \mathbf{y}) \boldsymbol{\nabla}_{\mathbf{y}} \psi(\mathbf{x}, \mathbf{y})\right] d Y\right\} \\
\mathbf{e}_{H} & =\frac{1}{|Y|}\left\{\int_{Y}\left[\left(\mathbf{I}+\boldsymbol{\partial}_{\mathbf{y}} \boldsymbol{\chi}(\mathbf{x}, \mathbf{y})\right): \mathbf{e}(\mathbf{x}, \mathbf{y})-\boldsymbol{\varepsilon}^{s}(\mathbf{x}, \mathbf{y}) \boldsymbol{\nabla}_{\mathbf{y}} \psi(\mathbf{x}, \mathbf{y})\right] d Y\right\}= \\
& =\frac{1}{|Y|}\left\{\int_{Y}\left[\mathbf{c}^{E}(\mathbf{x}, \mathbf{y}): \boldsymbol{\partial}_{\mathbf{y}} \mathbf{\Phi}(\mathbf{x}, \mathbf{y})+\mathbf{e}(\mathbf{x}, \mathbf{y})\left(\mathbf{I}+\boldsymbol{\nabla}_{\mathbf{y}} R(\mathbf{x}, \mathbf{y})\right)\right] d Y\right\} \\
\varepsilon_{H}^{S} & =\frac{1}{|Y|}\left\{\int_{Y}\left[\boldsymbol{\varepsilon}^{s}(\mathbf{x}, \mathbf{y})\left(\mathbf{I}+\boldsymbol{\nabla}_{\mathbf{y}} R(\mathbf{x}, \mathbf{y})\right)-\boldsymbol{\partial}_{\mathbf{y}} \mathbf{\Phi}(\mathbf{x}, \mathbf{y}): \mathbf{e}(\mathbf{x}, \mathbf{y})\right] d Y\right\}
\end{aligned}
$$

e

$$
\mathbf{b}_{H}=\frac{1}{|Y|} \int_{Y} \mathbf{b}(\mathbf{x}, \mathbf{y}) d Y \quad \rho_{H}=\frac{1}{|Y|} \int_{Y} \rho(\mathbf{x}, \mathbf{y}) d Y
$$

pode-se reescrever as Eq. (C.28) e (C.29) como: 


$$
\begin{aligned}
& \int_{\Omega} \partial_{\mathbf{x}} \delta \mathbf{u}_{0}(\mathbf{x}): \mathbf{c}_{H}^{E}: \partial_{\mathbf{x}} \mathbf{u}_{0}(\mathbf{x}) d \Omega+\int_{\Omega} \partial_{\mathbf{x}} \delta \mathbf{u}_{0}(\mathbf{x}): \mathbf{e}_{H} \nabla_{\mathbf{x}} \varphi_{0}(\mathbf{x}) d \Omega- \\
&-\int_{\Omega} \mathbf{b}_{H} \delta \mathbf{u}_{0}(\mathbf{x}) d \Omega-\int_{\Gamma} \mathbf{t} \delta \mathbf{u}_{0}(\mathbf{x}) d \Gamma++\int_{\Omega} \rho_{H} \ddot{\mathbf{u}}_{0}(\mathbf{x}) \delta \mathbf{u}_{0}(\mathbf{x}) d \Omega=0 \\
& \int_{\Omega} \partial_{\mathbf{x}} \delta \mathbf{u}_{0}(\mathbf{x}): \mathbf{e}_{H} \nabla_{\mathbf{x}} \delta \phi_{0}(\mathbf{x}) d \Omega-\int_{\Omega} \nabla_{\mathbf{x}} \phi_{0}(\mathbf{x}) \boldsymbol{\varepsilon}_{H}^{S} \nabla_{\mathbf{x}} \delta \phi_{0}(\mathbf{x}) d \Omega+ \\
&+\int_{\Gamma} d \delta \phi_{0}(\mathbf{x}) d \Gamma=0
\end{aligned}
$$

para qualquer valor admissível de $\delta \mathbf{u}_{0}(\mathbf{x})$ e $\delta \phi_{0}(\mathbf{x})$.

Utilizando as Eqs. (C.20) a (C.23), pode-se reescrever as Eqs. (C.30), (C.31) e (C.32) na forma:

$$
\begin{aligned}
\mathbf{c}_{H}^{E}= & \frac{1}{|Y|}\left\{\int _ { Y } \left[\left(\mathbf{I}+\partial_{\mathbf{y}} \boldsymbol{\chi}(\mathbf{x}, \mathbf{y})\right): \mathbf{c}^{E}(\mathbf{x}, \mathbf{y}):\left(\mathbf{I}+\partial_{\mathbf{y}} \boldsymbol{\chi}(\mathbf{x}, \mathbf{y})\right)+\right.\right. \\
& \left.\left.+\left(\mathbf{I}+\partial_{\mathbf{y}} \boldsymbol{\chi}(\mathbf{x}, \mathbf{y})\right): \mathbf{e}(\mathbf{x}, \mathbf{y}) \nabla_{\mathbf{y}} \psi(\mathbf{x}, \mathbf{y})\right] d Y\right\} \\
\mathbf{e}_{H}= & \frac{1}{|Y|}\left\{\int _ { Y } \left[\left(\mathbf{I}+\partial_{\mathbf{y}} \boldsymbol{\chi}(\mathbf{x}, \mathbf{y})\right): \mathbf{e}(\mathbf{x}, \mathbf{y})\left(\mathbf{I}+\nabla_{\mathbf{y}} R(\mathbf{x}, \mathbf{y})\right)-\right.\right. \\
& \left.\left.-\left(\mathbf{I}+\nabla_{\mathbf{y}} R(\mathbf{x}, \mathbf{y})\right) \boldsymbol{\varepsilon}^{S}(\mathbf{x}, \mathbf{y}) \nabla_{\mathbf{y}} \psi(\mathbf{x}, \mathbf{y})\right] d Y\right\} \\
\boldsymbol{\varepsilon}_{H}^{S}= & \frac{1}{|Y|}\left\{\int _ { Y } \left[\left(\mathbf{I}+\nabla_{\mathbf{y}} R(\mathbf{x}, \mathbf{y})\right) \boldsymbol{\varepsilon}^{S}(\mathbf{x}, \mathbf{y})\left(\mathbf{I}+\nabla_{\mathbf{y}} R(\mathbf{x}, \mathbf{y})\right)-\right.\right. \\
& \left.\left.-\partial_{\mathbf{y}} \mathbf{\Phi}(\mathbf{x}, \mathbf{y}): \mathbf{e}(\mathbf{x}, \mathbf{y})\left(\mathbf{I}+\nabla_{\mathbf{y}} R(\mathbf{x}, \mathbf{y})\right)\right] d Y\right\}
\end{aligned}
$$

ou, utilizando a notação indexada:

$$
\begin{aligned}
c_{r s p q}^{H}(\mathbf{x})= & \frac{1}{|Y|}\left\{\int _ { Y } \left[c_{i j k l}^{E}(\mathbf{x}, \mathbf{y})\left(\delta_{i p} \delta_{j q}+\frac{\partial \chi_{i}^{(p q)}}{\partial y_{j}}\right)\left(\delta_{k r} \delta_{l s}+\frac{\partial \chi_{k}^{(r s)}}{\partial y_{l}}\right)+\right.\right. \\
& \left.\left.+e_{k i j}(\mathbf{x}, \mathbf{y})\left(\delta_{i p} \delta_{j q}+\frac{\partial \chi_{i}^{(p q)}}{\partial y_{j}}\right) \frac{\partial \psi^{(r s)}}{\partial y_{k}}\right] d Y\right\} \\
e_{p r s}^{H}(\mathbf{x})= & \frac{1}{|Y|}\left\{\int _ { Y } \left[e_{k i j}(\mathbf{x}, \mathbf{y})\left(\delta_{k p}+\frac{\partial R^{(p)}}{\partial y_{k}}\right)\left(\delta_{i r} \delta_{j s}+\frac{\partial \chi_{i}^{(r s)}}{\partial y_{j}}\right)+\right.\right. \\
& \left.\left.-e_{k i j}(\mathbf{x}, \mathbf{y}) \frac{\partial \Phi_{i}^{(p)}}{\partial y_{j}} \frac{\partial \psi^{(r s)}}{\partial y_{k}}\right] d Y\right\} \\
\varepsilon_{p q}^{H}(\mathbf{x})= & \frac{1}{|Y|}\left\{\int _ { Y } \left[\varepsilon_{i j}^{S}(\mathbf{x}, \mathbf{y})\left(\delta_{i p}+\frac{\partial R^{(p)}}{\partial y_{i}}\right)\left(\delta_{j q}+\frac{\partial R^{(q)}}{\partial y_{j}}\right)+\right.\right.
\end{aligned}
$$




$$
\left.\left.-e_{k i j}(\mathbf{x}, \mathbf{y})\left(\delta_{k p}+\frac{\partial R^{(p)}}{\partial y_{k}}\right) \frac{\partial \Phi_{i}^{(q)}}{\partial y_{j}}\right] d Y\right\}
$$

e $c_{i j k l}^{H}=c_{k l i j}^{H}=c_{j i k l}^{H}, e_{i j k}^{H}=e_{i k j}^{H}, \varepsilon_{i j}^{H}=\varepsilon_{j i}^{H}$.

Os coeficientes piezelétricos homogeneizados dependem do tipo de classe cristalina considerada para o material piezelétrico básico. No caso desse estudo é considerada a classe hexagonal $6 \mathrm{~mm}$. Para simular a gradação contínua no interior dos elementos, as Eq. (C.18) e (C.19) devem ser resolvidas utilizando o conceito de elementos finitos gradados da Eq. (2.18).

\section{C.2 Condições de simetria}

Caso a célula unitária possua alguma simetria relativa aos eixos $x$ e $z$, é possível reduzir o custo computacional. Neste caso, o processo de otimização e homogeneização é realizado apenas em uma parte do domínio de projeto.

No caso do estado plano de deformação, se a célula unitária for simétrica em relação a um ou dois eixos os coeficientes homogeneizados serão ao menos ortotrópicos, uma vez que o material piezelétrico é ortotrópico e está presente na célula unitária. Se não houver nenhuma simetria os coeficientes homogeneizados serão anisotrópicos. Se o piezocompósito for anisotrópico, o número de coeficientes homogeneizados é reduzido para quatro coeficientes elásticos e dois coeficientes dielétricos para o caso 2D. Já os coeficientes homogeneizados piezelétricos dependem do tipo de classe cristalina do material piezelétrico de base. Para a classe hexagonal $6 \mathrm{~mm}$ considerada neste estudo, existem três coeficientes homogeneizados piezelétricos para o caso 2D.

As condições de simetria são impostas nas funções características $\boldsymbol{\chi}, \boldsymbol{\psi}, \Phi$ e $R$, apresentadas na Eq. (C.16). No estado plano de deformação existem cinco casos de carregamento a serem resolvidos no problema de homogeneização. Para cada um desses casos são apresentadas as condições de simetria para as funções características (deslocamentos e potenciais elétricos) nas Tabelas C.1 e C.2. Estas condições de contorno foram obtidas ampliando as condições de contorno de microestruturas elásticas (SILVA et al., 1999; SILVA; FONSECA; KIKUCHI, 1997; SILVA et al., 1999) para o caso piezelétrico a partir das Eq. (C.39), (C.40) e (C.41). Estas condições de contorno assumem que a célula unitária é ortotrópica com eixo de polarização piezelétrica na direção z (ou 3). Estas condições de contorno estão ilustradas na Seção 2.3a. 
Tabela C.1: Condições de contorno em $x=0$ e $x=Y_{1}$.

\begin{tabular}{|c|c|}
\hline Casos & Condições de Contorno em $x=0$ e $x=Y_{1}$ \\
\hline 1) $m=n(1$ ou 3$)$ & $\chi_{1}^{(m n)}=0 ; \quad T_{31}=0 ; \quad \frac{\partial \psi^{(m n)}}{\partial z_{1}}=0$ \\
\hline 2) $m n=13($ ou 31$)$ & $\chi_{3}^{(13)}=0 ; \quad T_{11}=0$ \\
\hline 3) $m=1$ & $\Phi_{3}^{(1)}=0 ; \quad T_{11}=0 ; \quad R^{(1)}=0$ \\
\hline 4) $m=3$ & $\Phi_{1}^{(3)}=0 ; \quad T_{31}=0 ; \quad \frac{\partial R^{(3)}}{\partial z_{1}}=0$ \\
\hline
\end{tabular}

Tabela C.2: Condições de contorno em $z=0$ e $z=Y_{3}$.

\begin{tabular}{|c|c|}
\hline Casos & Condições de Contorno em $z=0$ e $z=Y_{3}$ \\
\hline 1) $m=n(1$ ou 3$)$ & $\chi_{3}^{(m n)}=0 ; \quad T_{13}=0 ; \quad \psi^{(m n)}=0$ \\
\hline 2) $m n=13($ ou 31$)$ & $\chi_{1}^{(13)}=0 ; \quad T_{33}=0 ; \quad \frac{\partial \psi^{(13)}}{\partial y_{3}}=0$ \\
\hline 3) $m=1$ & $\Phi_{1}^{(1)}=0 ; \quad T_{33}=0 ; \quad \frac{\partial R^{(1)}}{\partial y_{3}}=0$ \\
\hline 4) $m=3$ & $\Phi_{3}^{(3)}=0 ; \quad T_{13}=0 ; \quad R^{(3)}=0$ \\
\hline
\end{tabular}




\section{Apêndice D - Verificação da Implementação do Método de Homogeneização}

Para verificar se a implementação do Método de Homogeneização está correta, é realizado um estudo de sensibilidade do piezocompósito 2-2 composto por PZT-5A e epóxi, variando-se a fração de volume dos seus materiais constituintes na célula unitária (ver Fig. D.1b), e comparando com resultados disponíveis na literatura científica (SILVA et al., 1999). As medidas que descrevem o desempenho de um compósito são obtidas considerando apenas as propriedades constitutivas (os efeitos de inércia são desprezados), conforme descrito por Smith (SMITH, 1993) e Smith e Auld (SMITH; AULD, 1991). São quatro as principais características de desempenho de materiais piezocompósitos (AVELLANEDA; SWART, 1994; SILVA; FONSECA; KIKUCHI, 1997):

-Coeficiente de acoplamento hidrostático $\left(d_{h}\right)$ :

$$
d_{h}=\frac{\left\langle\mathbf{D}_{3}\right\rangle}{P}=d_{33}+d_{23}+d_{13}
$$

-Figura de mérito $\left(d_{h} g_{h}\right)$ :

$$
g_{h}=\frac{\left\langle E_{3}\right\rangle}{P}=\frac{d_{h}}{\varepsilon_{33}^{T}} \Rightarrow d_{h} g_{h}=\frac{d_{h}^{2}}{\varepsilon_{33}^{T}}
$$

-Fator de acoplamento eletromecânico hidrostático $\left(k_{h}\right)$ :

$$
k_{h}=\sqrt{\frac{d_{h}^{2}}{\varepsilon_{33}^{T} s_{h}^{E}}}
$$

- Fator de acoplamento eletromecânico $\left(k_{t}\right)$ : 


$$
k_{t}=\sqrt{\frac{e_{33}^{2}}{c_{33}^{D} \varepsilon_{33}^{S}}}
$$

onde $s_{h}^{E}=\left(\left\langle\varepsilon_{1}\right\rangle+\left\langle\varepsilon_{2}\right\rangle+\left\langle\varepsilon_{3}\right\rangle\right) / P$ e $c_{33}^{D}=c_{33}^{E}+\left(e_{33}\right)^{2} / \varepsilon_{33}^{S}$.

Os valores $d_{h}$ e $g_{h}$ medem a resposta do material em termos da carga e voltagem elétricas geradas, respectivamente, quando sujeito a um campo de pressão hidrostático, considerando um campo elétrico nulo $\left(\left\langle E_{3}\right\rangle=0\right.$, em condições de curto-circuito) e deslocamento elétrico nulo $\left(\left\langle D_{3}\right\rangle=0\right.$ em condições de circuito aberto), respectivamente. $d_{h} g_{h}$ é o produto de $d_{h}$ e $g_{h}$. O coeficiente $k_{h}$ mede a conversão acústica/elétrica. As expressões de $d_{h}$ e $s_{h}^{E}$ podem ser obtidas substituindo a pressão hidrostática na Eq. (A.4), e considerando um campo elétrico nulo $\left(\left\langle E_{3}\right\rangle=0\right)$. A expressão de $g_{h}$ pode ser obtida da mesma forma, mas considerando um deslocamento elétrico nulo $\left(\left\langle D_{3}\right\rangle=0\right)$ na Eq. (A.3). O operador $\langle$.$\rangle indica quantidades médias volumétricas \frac{1}{|V|} \int_{V} . d V$ (ver Apêndice A). Estas quatro características de desempenho estão relacionadas à conversão de energia, sendo que as três primeiras, Eqs. (D.1), (D.2) e (D.3), referem-se a aplicações estáticas e a quarta, Eq. (D.4), à aplicações dinâmicas.

Os gráficos da Fig. D.2 mostram a variação das características de desempenho em função da fração de volume de PZT-5A e os valores máximos estão listados na Tabela D.1. A partir destes gráficos pode-se ver que os valores máximos de cada característica de desempenho são obtidos para frações de volume distintas. Além disso, os gráficos obtidos são iguais aos apresentados por Silva et al. (SILVA et al., 1999), validando assim o programa implementado.

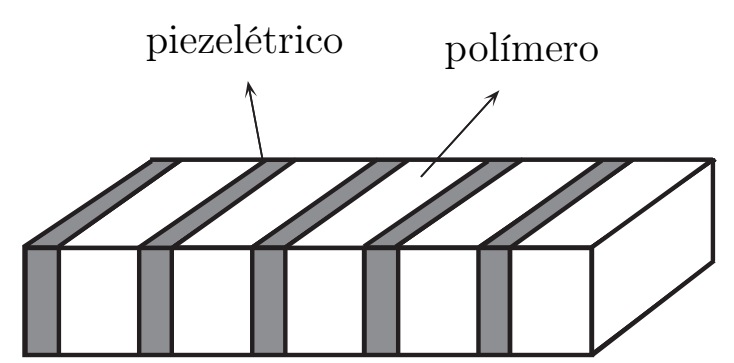

(a)

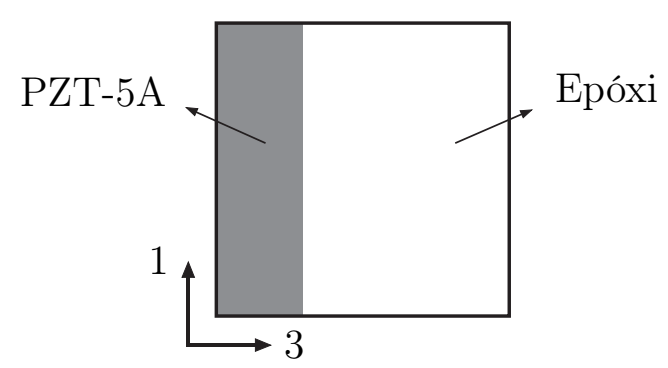

(b)

Figura D.1: (a) Exemplo de um piezocompósito 2-2; (b) Exemplo de célula unitária do piezocompósito 2-2.

Tabela D.1: Valores máximos das características de desempenho dos materiais PZT-5A e piezocompósito 2-2 (PZT-5A/Epóxi).

\begin{tabular}{|c|c|c|c|c|}
\hline Célula Unitária & $d_{h}(p C / N)$ & $d_{h} g_{h}\left(\mathrm{pm}^{2} / N\right)$ & $k_{h}$ & $k_{t}$ \\
\hline \hline PZT-5A (puro) & 68,2 & 0,222 & 0,145 & 0,361 \\
\hline piezocompósito 2-2 & 93,5 & 3,452 & 0,145 & 0,472 \\
\hline
\end{tabular}




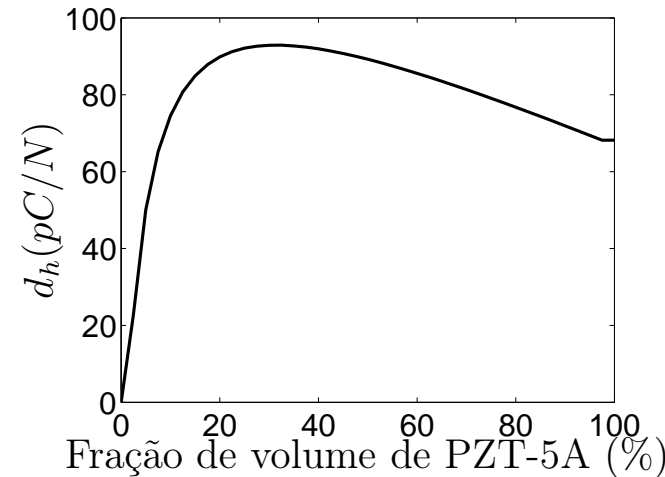

(a)

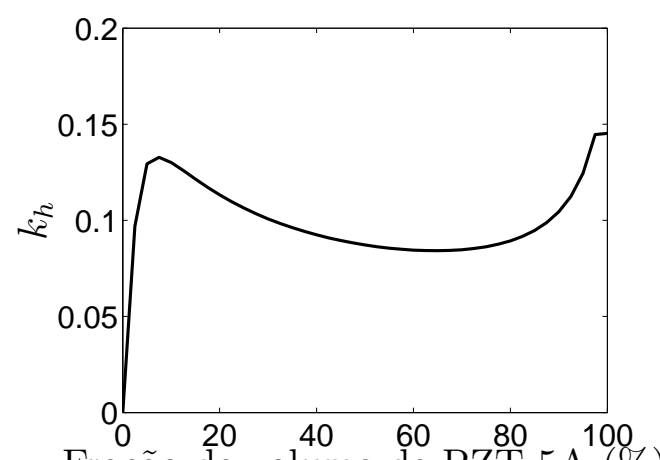

Fração de volume de PZT-5A (\%)

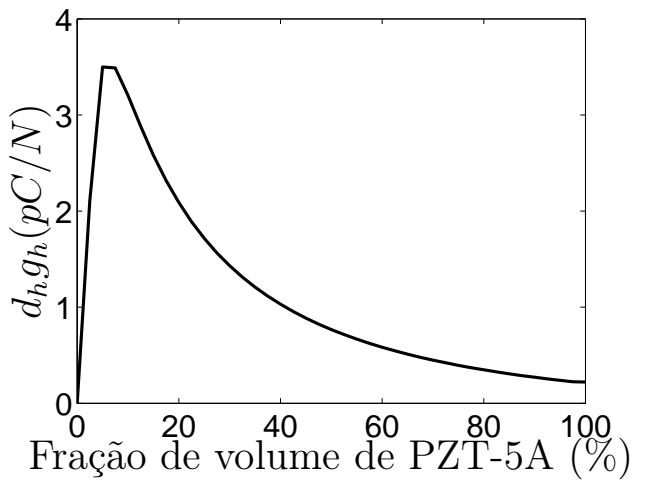

(b)

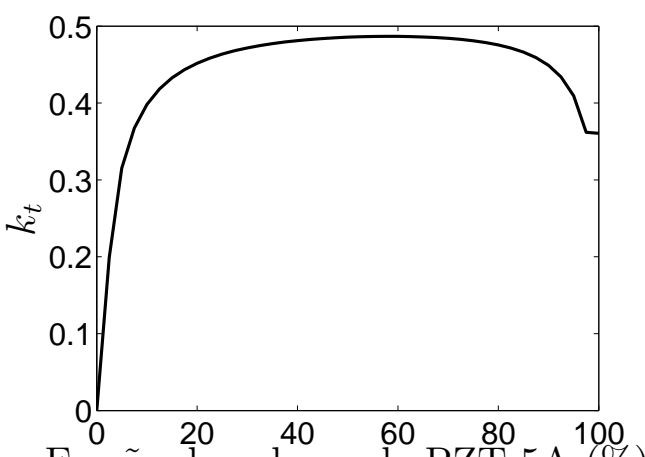

(c)

(d)

Figura D.2: Piezocompósito 2-2: caracterização de desempenho em função da fração de volume de PZT-5A. 


\section{Apêndice E - Método de Otimização Topológica (MOT)}

O MOT baseado na abordagem micro se baseia em dois conceitos fundamentais: o domínio estendido fixo de projeto e o modelo de material. A seguir, cada um destes conceitos são brevemente descritos nas Seções E.1 e E.2. Alguns aspectos importantes na implementação do MOT, tais como não-unicidade de solução, dependência de malha e instabilidade de tabuleiro de xadrez, são discutidos na Seção E.3.

\section{E.1 Domínio Estendido Fixo de Projeto}

No MOT não são alterados os contornos externos do domínio da estrutura durante o processo de otimização. Esse domínio fixo durante a otimização que conterá a estrutura desconhecida, e que se encontra limitado pelos pontos de apoio da estrutura e os pontos de aplicação dos carregamentos, denomina-se domínio estendido fixo de projeto, $\Omega$. Assim, no MOT é possível remover e adicionar material dentro do espaço definido pelo domínio fixo na busca da topologia ótima, mas a sua forma inicial não é alterada. A Fig. E.1 ilustra este conceito. Sendo assim, o MOT pode ser observado como um método que encontra a função de distribuição ótima de dois ou mais materiais no interior de um domínio de projeto fixo.

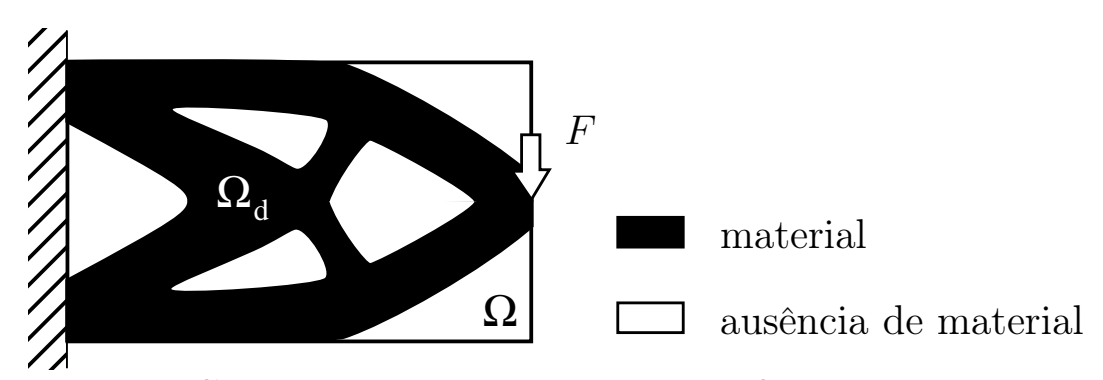

Figura E.1: Conceito de domínio estendido fixo.

Na implementação numérica do módulo de análise, o domínio estendido fixo deve ser discretizado em elementos finitos que permanecerão inalterados durante o processo de otimização, sendo alterada somente a distribuição de material nos elementos. Isto facilita 
a implementação da análise de sensibilidade, uma vez que vale para todos os elementos do domínio estendido fixo a relação geral do cálculo da sensibilidade:

$$
\frac{\partial}{\partial A_{n}} \int_{\Omega} q d \Omega=\int_{\Omega} \frac{\partial q}{\partial A_{n}} d \Omega
$$

onde $A_{n}$ é a variável de projeto do elemento $n$ e $q$ é uma função contínua e diferenciável.

\section{E.2 Modelo de Material}

No MOT geralmente dois tipos de materiais são utilizados para determinar a topologia ótima da estrutura. Essa distribuição de materiais pode ser definida como um problema de otimização de parâmetros discretos ou um problema do tipo 0 - 1. Assim, o problema é definido por uma função discreta $\rho(x, y)=\chi(x, y) \rho_{0}$ (onde $\rho_{0}$ é a propriedade do material a ser distribuído) definida em cada coordenada Cartesiana $(x, y)$ de um domínio de projeto fixo bidimensional, $\Omega$, da seguinte forma:

$$
\chi(x, y)=\left\{\begin{array}{lll}
1 & \text { se } & x, y \in \Omega_{d} \\
0 & \text { se } & x, y \in \Omega \backslash \Omega_{d}
\end{array}\right.
$$

A utilização de valores discretos para a variação de material dentro do domínio de projeto fixo torna o problema mal posto, ou seja, sem solução. A solução para o problema anteriormente mencionado é a relaxação do problema de otimização, permitindo-se que o material, em cada ponto do domínio de projeto, assuma valores intermediários, ao se passar da condição de ausência de material a sólido. Assim, define-se o conceito do modelo de material, o qual está relacionado com a forma de variar-se o material em cada ponto do domínio de projeto fixo, entre zero (geralmente ar) e um (sólido). A definição do modelo de material define o grau de relaxação do problema.

Existem vários modelos de material descritos na literatura que podem ser utilizados no MOT. Entre os principais destacam-se: o método de homogeneização (HASSANI; HINTON, 1998a, 1998b, 1998c), e o método das densidades (ROZVANY, 1992, 2001; BENDSøE; SIGMUND, 2003).

\section{Método das Densidades}

O método das densidades tem obtido a preferência dos pesquisadores da área de OT por ser um modelo relativamente simples de implementar, em relação ao método de 
homogeneização, e por utilizar, geralmente, uma única variável de projeto: a densidade relativa do material (pseudo-densidade) em cada ponto do domínio estendido fixo de projeto. Nas implementações do MOT, comumente utiliza-se a formulação SIMP (Simple Isotropic Material with Penalization). O SIMP consiste em uma equação matemática que define o valor da propriedade do material em cada ponto do domínio em função de uma pseudo-densidade (que nos problemas de OT geralmente é a variável de projeto) e a propriedade básica do material a ser distribuído. Este modelo de material pode ser expresso como:

$$
E^{H}(x, y)=\rho(x, y) E^{0}
$$

onde $E^{0}$ é o tensor que representa as propriedades do material base e $\rho(x, y)$ é interpretada como uma função de distribuição contínua de densidade ou variável de projeto, a qual está definida no intervalo $0 \leq \rho(x, y) \leq 1$. Os termos $x$ e $y$ são as coordenadas Cartesianas de um ponto qualquer dentro do domínio de projeto bidimensional $\Omega$, e $p$ e o fator de penalidade da variável de projeto, o qual determina a natureza do problema: continua ou discreta.

No modelo de material SIMP existe um conflito na magnitude do fator de penalização, p. Assim, com a utilização de um $p$ pequeno (natureza mais contínua do que discreta do problema) geram-se as escalas de cinza ou valores de propriedades intermediárias de material (valores de propriedades entre dois materiais base; por exemplo, aço e PZT). Por outro lado, ao utilizarem-se valores de $\mathrm{p}$ muito altos; por exemplo, $p=9$ (o que representa uma natureza mais discreta do que contínua), pode-se recuperar problemas de convergência e de instabilidade numérica, devido à tendência de retornar ao problema discreto (limitar os valores da densidade de cada elemento somente a 0 ou 1 ).

A aproximação SIMP já foi chamada de aproximação de "material fictício", pois se acreditava que nenhum material alcançaria as propriedades de material dadas pela Eq. (E.3). No entanto, com o surgimento, nas duas últimas décadas, do conceito de materiais MGF a idéia de "material fictício" tem sido superada, uma vez que esses materiais possuem valores de propriedades intermediárias (escalas de cinza) atualmente fabricáveis. Por outro lado, para o caso de materiais não-MGF, Bendsøe e Sigmund (BENDSøE; SIGMUND, 1999) provaram que a aproximação SIMP é fisicamente possível desde que uma simples condição sobre o fator $p$ seja satisfeita (em problemas estruturais): o fator de penalidade deve ser maior ou igual a 3 .

No projeto de materiais MGF, onde dois ou mais materiais são misturados, e onde 
o surgimento das escalas de cinza é desejado, o modelo de material SIMP, da Eq. (E.3), pode ser expresso como (CARBONARI; SILVA; PAULINO, 2007):

$$
E^{H}(x, y)=\rho(x, y) E_{1}+[1-\rho(x, y)] E_{2}
$$

onde $\rho(x, y)$ é uma função de distribuição contínua de pseudo-densidade ou variável de projeto, definida no intervalo $0 \leq \rho(x, y) \leq 1$, e $E_{1}$ e $E_{2}$ representam os tensores constitutivos de dois materiais base diferentes. Quando o valor da pseudo-densidade $\rho$ é igual a 1 , temos que $E^{H}=E_{1}$ e quando o valor de pseudo-densidade é igual a $0, E^{H}=E_{2}$. Valores intermediários do valor da pseudo-densidade $\rho$ geram o tensor constitutivo de um material MGF. Geralmente, a Eq. (E.4) é denominada modelo Voigt ou lei da mistura para materiais rígidos, amplamente conhecida na área de materiais compósitos para o cálculo das suas propriedades efetivas.

\section{E.3 Aspectos Numéricos do MOT}

Na aplicação do MOT existem alguns problemas resultantes próprios da natureza discreta e do caráter computacional do método. Entre os principais pode-se mencionar a dependência da solução da discretização e instabilidade de tabuleiro de xadrez, os quais são brevemente descritos a seguir. Esses problemas são descritos mais detalhadamente em (BENDSØE; SIGMUND, 2003).

\section{E.3.1 Dependência de malha}

O problema da dependência de malha consiste na obtenção de diferentes soluções para diferentes discretizações do domínio de projeto fixo e tamanho de malha. Assim, o refinamento da malha de elementos finitos deveria resultar em uma melhor aproximação do problema real e não numa estrutura diferente como efetivamente acontece em problemas solucionados mediante o MOT. Varias técnicas têm sido utilizadas para reduzir este problema, entre as principais, incluem-se aumento de restrições no problema de otimização, ou utilização de filtros na implementação (BENDSøE; SIGMUND, 2003).

O problema de dependência da malha pode ser dividido em duas categorias, conforme sua origem. Para os casos onde sempre se obtém topologias diferentes, a medida que se aumenta a discretização da malha, o problema de dependência da malha é a manifestação numérica da não-existência de solução do problema. Existe também a possibilidade do problema não apresentar unicidade de solução. Essa situação pode ocorrer conforme as 
condições de contorno aplicadas ao problema. Por exemplo, no caso de uma barra sob tração uniaxial, as soluções de uma única barra de maior diâmetro ou de diversas barras de diâmetro menor, porém com a mesma área da barra única, apresentam a mesma rigidez (SIGMUND; PETERSSON, 1998).

Uma vez que a dependência da malha é uma manifestação numérica do fato do problema discreto de parâmetros distribuídos não possuir solução (ser mal-posto), uma forma de se evitar a dependência de malha é através da relaxação desse problema, tornando-o bem-posto. Essa é uma solução "natural" na implementação do MOT no projeto de estruturas MGF, uma vez que permite o surgimento de materiais com propriedades intermediárias (escala de cinza). O problema de dependência de malha também pode ser resolvido através da utilização de filtros espaciais. Os filtros substituem a possível função não-regular por uma função regularizada, que é obtida através da convolução desta com uma função suave (BOURDIN, 2001). Os filtros suavizam a distribuição espacial das variáveis de projeto ao longo do domínio estendido fixo, através de uma transformação matemática de cada variável do problema de otimização. Alguns trabalhos da literatura sugerem a aplicação do filtro sobre o campo de pseudo-densidades (SIGMUND, 2000b), mas como essas pseudo-densidades geralmente são as variáveis de projeto, atualizadas a cada iteração durante o processo de otimização, ao aplicar esse filtro modificam-se os valores ótimos encontrados para essas variáveis, influenciando a otimização realizada. Outras abordagens aplicam a técnica de filtragem diretamente sobre os limites móveis na Programação Linear Sequencial - PLS (CARDOSO; FONSECA, 2003), o qual permite obter um controle sobre o gradiente da variável do problema sem impor uma mudança artificial sobre a distribuição das densidades, obtidas da otimização. Porém, essa abordagem influencia a convergência do método.

Para que a convergência da otimização não seja comprometida, Bruns e Tortorelli (BRUNS; TORTORELLI, 2001) propuseram uma técnica de projeção onde a pseudo-densidade de cada nó do domínio é uma função que depende das variáveis de projeto associadas a cada nó pertencente ao filtro e, além disso, depende das distâncias entre o nó central e cada nó dentro de um círculo de raio $r_{\text {min }}$ (ver a Fig. 2.20). Este tipo de abordagem não influi na convergência do método de otimização, pois somente está alterando as pseudo-densidades e não seus limites móveis, e ainda garante a independência da malha da solução ótima. O Capítulo 2 apresenta em detalhe a técnica de projeção inicialmente apresentada por Bruns e Tortorelli (BRUNS; TORTORELLI, 2001). 


\section{E.3.2 Instabilidade de tabuleiro de xadrez}

A instabilidade de xadrez é caracterizada pela formação de regiões com material (ilustrado com elementos de cor escura) e elementos sem material (ilustrados na cor branca), dispostos em forma de tabuleiro, como mostrado na Fig. E.2.

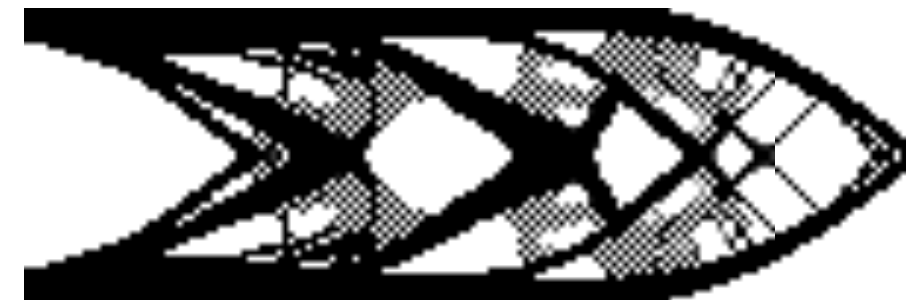

Figura E.2: Exemplo de instabilidade de tabuleiro de xadrez.

As publicações relacionadas com o estudo da instabilidade de xadrez sugerem duas formas distintas para sua eliminação nos problemas de OT, que tradicionalmente são empregadas pela literatura (DIAZ; SIGMUND, 1995; BOURDIN, 2001; BENDSØE; SIGMUND, 2003). Uma delas é aumentar a ordem do elemento finito e a outra é utilizar métodos de filtragem ou de controle de gradientes das densidades no domínio. A instabilidade xadrez é indesejável na solução do problema, pois não se configura numa distribuição ótima de material, mas num fenômeno que aparece devido à formulação (funções de interpolação do elemento finito utilizado no processo de otimização.

Foi demonstrado em estudos realizados por Jog et al. (JOG; HABER; BENDSOE, 1994), Diaz e Sigmund (DIAZ; SIGMUND, 1995) e Jog e Haber (JOG; HABER, 1996) que os problemas de instabilidades numéricas são inerentes a problemas de variacionais mistos. O problema de OT pode ser interpretado como um problema que contém um variacional misto envolvendo o campo de densidades (grau da função de interpolação de deslocamentos no MEF). Assim, dependendo do valor do fator de penalidade $p$, a instabilidade xadrez pode ocorrer mesmo utilizando elementos de 9 nós (Jog e Haber (JOG; HABER, 1996)). O trabalho de Jog et al. (JOG; HABER; BENDSOE, 1994) e Jog e Haber (JOG; HABER, 1996) propõem uma série de testes (baseados em observações práticas ou no estudo matemático do problema variacional misto) para avaliar se uma determinada combinação de interpolações de densidades e deslocamentos resulta em configurações instáveis ou estáveis. A formação da instabilidade xadrez não é um aspecto exclusivo da OT, este fenômeno também se manifesta na solução de MEF para diversos outros problemas com variacional misto. Um exemplo deste fenômeno é observado na distribuição de pressões obtidas através da análise de MEF do problema de escoamento de fluidos de Stokes (Oden et al. (ODEN; KIKUCHI; SONG, 1982)), cujo variacional envolve velocidades e pressões. Para determinadas combinações de interpolação para o campo de deslocamentos e para o campo de pressões, o problema se mostra instável. 
Portanto, é necessário encontrar uma forma alternativa para a eliminação da instabilidade xadrez nos problemas de OT. A introdução de métodos de controle da variação espacial das variáveis de projeto (pseudo-densidades) no domínio de projeto, através da implementação de filtros na otimização é uma das mais utilizadas pela literatura (BENDSØE; SIGMUND, 2003), uma vez que a instabilidade xadrez se caracteriza por variações bruscas nos gradientes das variáveis de projeto. Além disso, esse método também permite controlar de forma razoável a complexidade da topologia obtida pelo MOT (BOURDIN, 2001), bem como, a dependência de malha. Nesse sentido, recursos como a restrição de perímetro também se aplicam para o controle da instabilidade xadrez (HABER; JOG; BENDSøE, 1996). Sigmund (SIGMund, 2007) faz uma análise dos métodos para reduzir os problemas numéricos da OT e apresenta uma revisão completa sobre este assunto. Dentre os métodos estudados, Sigmund (SIGMUND, 2007) sugere a técnica de projeção (BRUNS; TORTORELLI, 2001). 